Supporting Information

\title{
Photochemical Organocatalytic Benzylation of Allylic C-H Bonds
}

\author{
Emilien Le Saux, ${ }^{\mathrm{a}}$ Margherita Zanini, ${ }^{\mathrm{a}}$ and Paolo Melchiorre*,a,b \\ ${ }^{a} I C I Q$ - Institute of Chemical Research of Catalonia \\ Av. Països Catalans 16, 43007 Tarragona, Spain \\ ' ICREA - Catalan Institution for Research and Advanced Studies \\ Pg. Lluís Companys 23, 08010 Barcelona, Spain \\ *Correspondence to: pmelchiorre@iciq.es
}




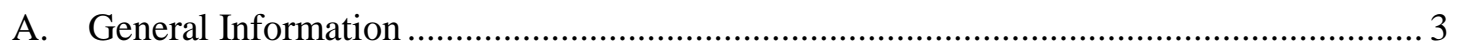

B. Moderately Successful and Unsuccessful Substrates .......................................................... 4

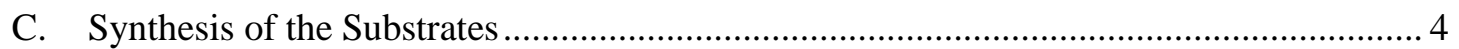

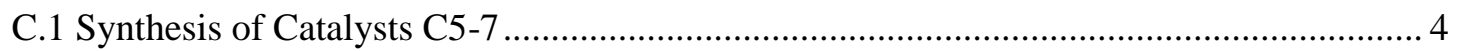

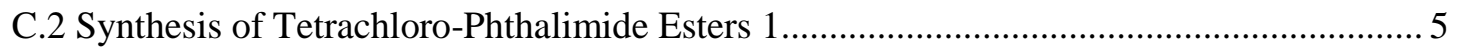

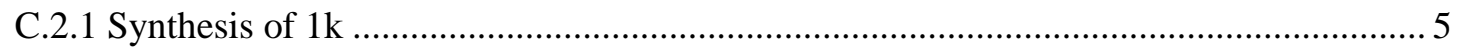

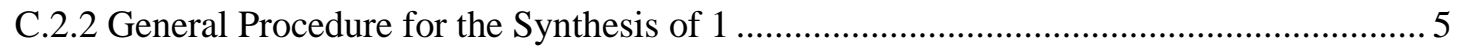

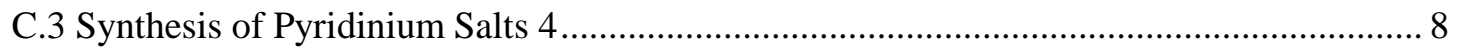

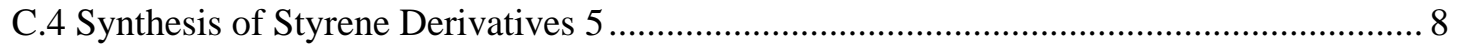

C.4.1 General Procedure for the Wittig Reaction ............................................................... 8

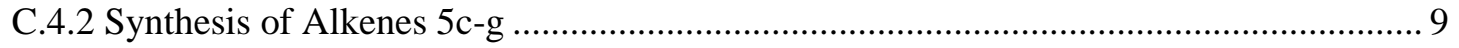

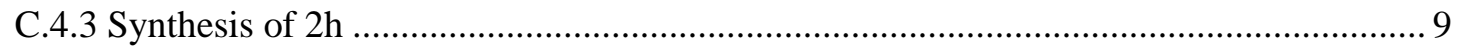

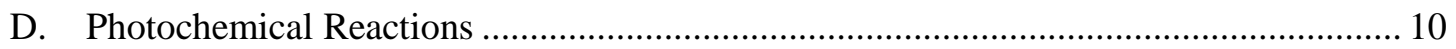

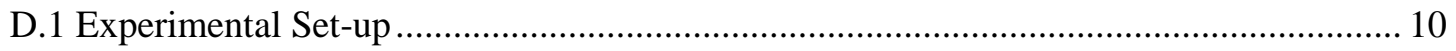

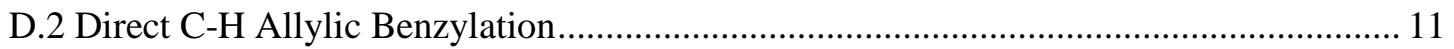

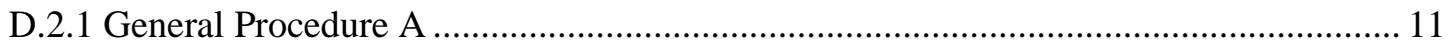

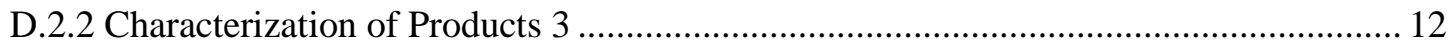

D.3 Three-component C-H Allylic Benzylation .................................................................... 16

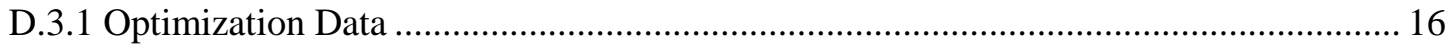

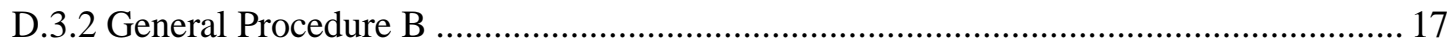

D.3.3 Characterization of Products 6 …....................................................................... 17

D.3.3 Telescoped Three-component C-H Allylic benzylation.................................................. 34

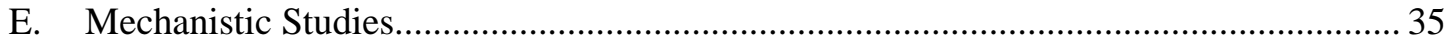

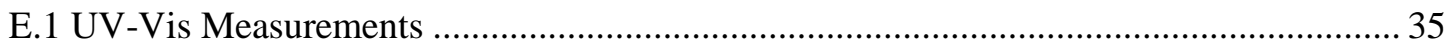

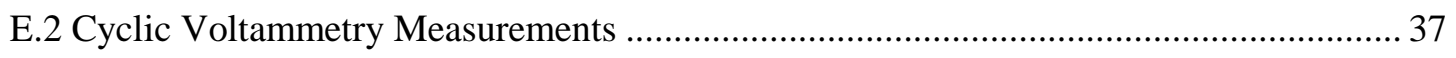

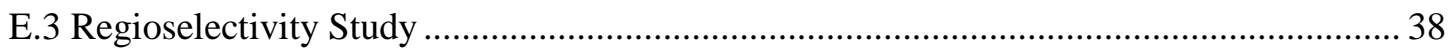

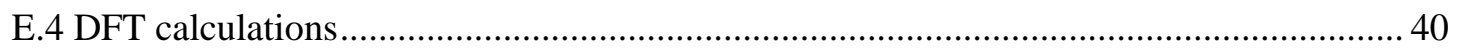

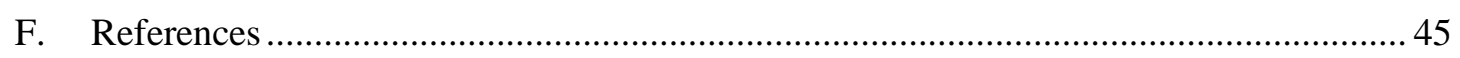




\section{A. General Information}

The NMR spectra were recorded at $300 \mathrm{MHz}, 400 \mathrm{MHz}$ and $500 \mathrm{MHz}$ for ${ }^{1} \mathrm{H}$ or at $75 \mathrm{MHz}, 101$ $\mathrm{MHz}$ and $126 \mathrm{MHz}$ for ${ }^{13} \mathrm{C}, 376 \mathrm{MHz}$ for ${ }^{19} \mathrm{~F}, 162 \mathrm{MHz}$ for ${ }^{31} \mathrm{P}$, respectively. The chemical shifts

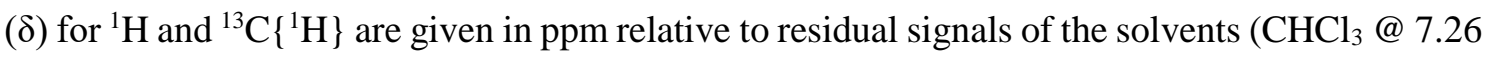
ppm ${ }^{1} \mathrm{H}$ NMR, $77.00 \mathrm{ppm}{ }^{13} \mathrm{C}$ NMR). Coupling constants are given in $\mathrm{Hz}$. The following abbreviations are used to indicate the multiplicity: s, singlet; $d$, doublet; t, triplet; q, quartet; $\mathrm{m}$, multiplet; br s, broad signal.

High-resolution mass spectra (HRMS) were obtained from the ICIQ High-Resolution Mass Spectrometry Unit on MicroTOF Focus and Maxis Impact (Bruker Daltonics) with electrospray ionization or atmospheric pressure chemical ionization. UV-vis measurements were carried out on a Shimadzu UV-2401PC spectrophotometer equipped with photomultiplier detector, double beam optics and D2 and $\mathrm{W}$ light sources.

The authors are indebted to the team of the Research Support Area at ICIQ, particularly to the $X$-ray, the NMR, and the High-Resolution Mass Spectrometry Units. Grace Fox is thanked for proofreading the manuscript.

General Procedures. All reactions were set up under an argon atmosphere in oven-dried glassware using standard Schlenk techniques, unless otherwise stated. Synthesis grade solvents were used as purchased. Anhydrous solvents were taken from a commercial SPS solvent dispenser. Chromatographic purification of products was accomplished using flash column chromatography (FC) on silica gel (35-70 mesh). For thin layer chromatography (TLC) analysis throughout this work, Merck precoated TLC plates (silica gel $60 \mathrm{GF}_{254}, 0.25 \mathrm{~mm}$ ) were used, using UV light as the visualizing agent and either phosphomolybdic acid in EtOH, dinitrophenylhydrazine in $\mathrm{EtOH} / \mathrm{H}_{2} \mathrm{O}, p$-anisaldehyde or basic aqueous potassium permanganate $\left(\mathrm{KMnO}_{4}\right)$, and heat as developing agents. Organic solutions were concentrated under reduced pressure on a Büchi rotary evaporator (in vacuo at $40^{\circ} \mathrm{C}, \sim 5 \mathrm{mbar}$ ).

Determination of Diastereomeric Ratio. The diastereomeric ratio was determined by ${ }^{1} \mathrm{H}$ NMR analysis of the crude reaction mixture through integration of diagnostic signals.

Materials: Commercial grade reagents and solvents were purchased at the highest commercial quality from Sigma Aldrich, Fluka, Acros Organics, Fluorochem, or Alfa Aesar and used as received, unless otherwise stated. Thiol catalysts $\mathbf{C 1}, \mathbf{C 2}$ and $\mathbf{C 3}$ are commercially available. The thiophosphoric acid $\mathbf{C 4}$ was prepared according to a reported procedure. ${ }^{1}$ 


\section{B. Moderately Successful and Unsuccessful Substrates}

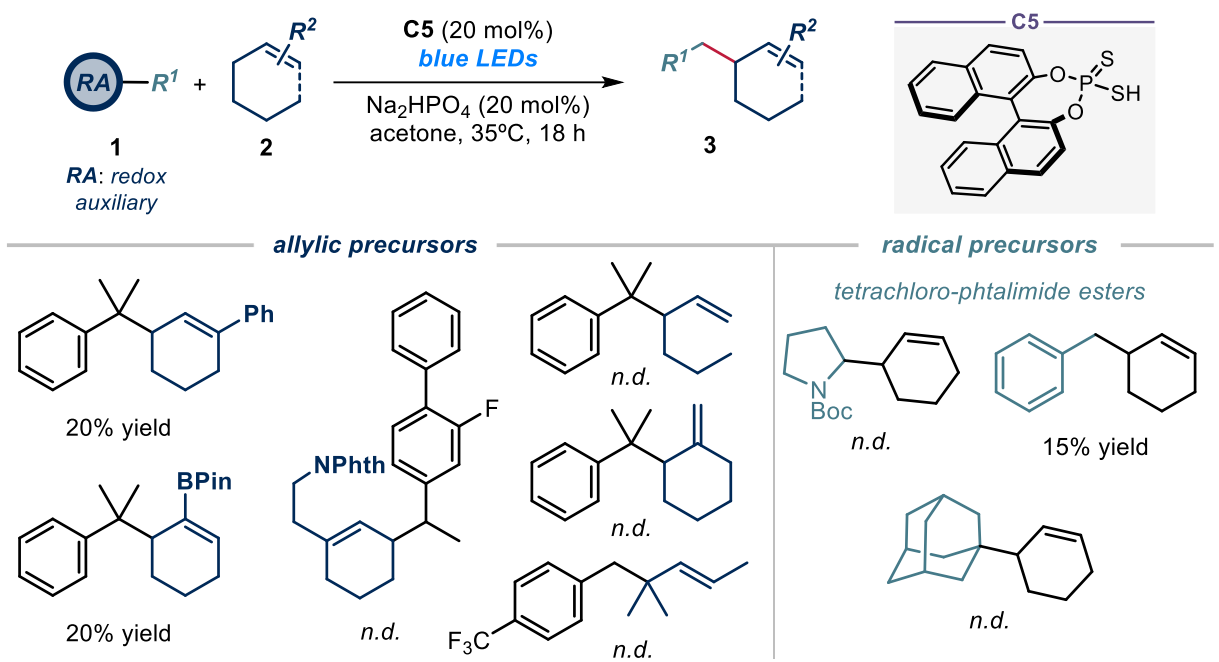

Figure S1. Moderately successful and unsuccessful substrates in the direct allylic benzylation. n.d.: not detected.

\section{Synthesis of the Substrates}

\section{C.1 Synthesis of Catalysts C5-7}

Catalysts C5-7 were prepared following a reported procedure, ${ }^{2}$ as described below.

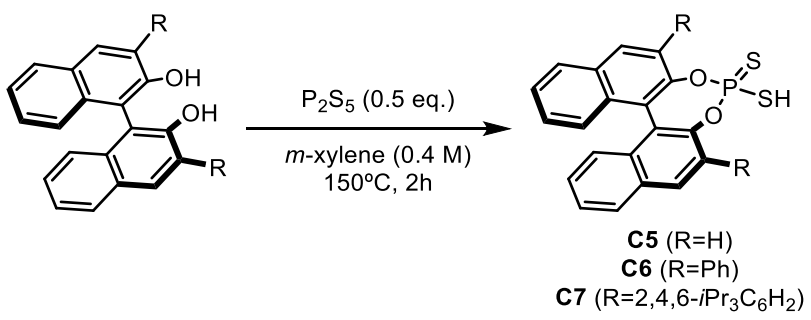

(11bS)-4-mercaptodinaphtho[2,1-d:1',2'-f][1,3,2]dioxaphosphepine 4-sulfide (C5): A flame dried flask was charged with (S)-1,1'-bi-2-naphtol (2.29 g, $8.0 \mathrm{mmol}, 1.0$ equiv), $\mathrm{P}_{2} \mathrm{~S}_{5}$ (889 mg, $4.0 \mathrm{mmol}, 0.5$ equiv), and anhydrous $m$-xylene $(20 \mathrm{~mL})$. The flask was equipped with a condenser and placed in an oil-bath preheated to $150{ }^{\circ} \mathrm{C}$. The progress of the reaction was monitored by disappearance of the phenolic protons, as inferred by ${ }^{1} \mathrm{H}$ NMR analysis of the crude mixture. After $2 \mathrm{~h}$, the reaction was completed and the mixture was cooled to ambient temperature. The solution was decanted into a flame-dried $100 \mathrm{~mL}$ flask and the solvent was evaporated in vacuo. The crude product was dissolved in anhydrous $\mathrm{CH}_{2} \mathrm{Cl}_{2}(5 \mathrm{~mL})$ and treated with hexanes $(50 \mathrm{~mL})$. At this point, a fine precipitate was observed. The solvent was then partially evaporated until about 2 to $3 \mathrm{~mL}$ solvent was left. The precipitate was then collected by filtration and washed with ice-cold hexanes to afford C5 (2.23g, $5.86 \mathrm{mmol}, 73 \%$ yield) as a white powder that displayed spectroscopic data consistent with those reported previously. ${ }^{3}$

${ }^{1} \mathrm{H} \mathrm{NMR}\left(400 \mathrm{MHz}, \mathrm{CDCl}_{3}\right) \delta 8.13-8.07(\mathrm{~m}, 2 \mathrm{H}), 8.00(\mathrm{dd}, J=8.1,1.2 \mathrm{~Hz}, 2 \mathrm{H}), 7.60(\mathrm{dd}, J=$ $8.8,1.4 \mathrm{~Hz}, 2 \mathrm{H}), 7.54(\mathrm{ddt}, J=8.0,6.8,1.1 \mathrm{~Hz}, 2 \mathrm{H}), 7.48-7.42(\mathrm{~m}, 2 \mathrm{H}), 7.36$ (ddd, $J=8.4,6.7$, $1.3 \mathrm{~Hz}, 2 \mathrm{H})$.

${ }^{13} \mathrm{C} \mathrm{NMR}\left(75 \mathrm{MHz}, \mathrm{CDCl}_{3}\right) \delta 147.2,132.4,132.0,131.1,128.6,127.2,126.9,126.1,122.6,121.1$. ${ }^{31} \mathrm{P} \mathrm{NMR}\left(162 \mathrm{MHz}, \mathrm{CDCl}_{3}\right) \delta 100.24$. 


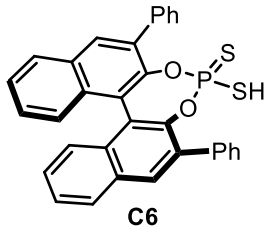

(11bS)-4-mercapto-2,6-diphenyldinaphtho[2,1-d:1',2'-

f] $[1,3,2]$ dioxaphosphepine 4-sulfide (C6)

Prepared according to the reported procedure using (S)-3,3'-diphenyl-[1,1'binaphthalene]-2,2'-diol.

$\underline{{ }^{1} \mathrm{H} \mathrm{NMR}}\left(500 \mathrm{MHz}, \mathrm{CDCl}_{3}\right) \delta 8.14(\mathrm{~s}, 2 \mathrm{H}), 8.06-8.02(\mathrm{~m}, 2 \mathrm{H}), 7.75-7.71$

${ }^{13} \mathrm{C} \mathrm{NMR}\left(101 \mathrm{MHz}, \mathrm{CDCl}_{3}\right) \delta 137.2,131.4,130.2,129.6,128.9,128.6,128.3,127.8,127.1$, $126.7,126.3$.

1ㅏ PMR $\left(202 \mathrm{MHz}, \mathrm{CDCl}_{3}\right) \delta 96.2$.

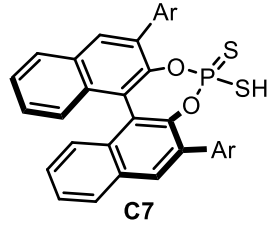

(Ar=2,4,6-i $\mathrm{Pr}_{3} \mathrm{C}_{6} \mathrm{H}_{2}$ )

(2r,11bS)-4-mercapto-2,6-bis(2,4,6-triisopropylphenyl)dinaphtho[2,1d:1',2'-f][1,3,2]dioxaphosphepine 4-sulfide (C7)

Prepared according to the reported procedure using (1'S,3r)-3,3'-bis $(2,4,6-$ triisopropylphenyl)-[1,1'-binaphthalene]-2,2'-diol.

${ }^{1} \mathrm{H} \mathrm{NMR}\left(500 \mathrm{MHz}, \mathrm{CDCl}_{3}\right) \delta 7.90-7.84(\mathrm{~m}, 4 \mathrm{H}), 7.45(\mathrm{t}, J=7.5 \mathrm{~Hz}, 2 \mathrm{H})$, 7.25 (ddd, $J=8.2,6.8,1.3 \mathrm{~Hz}, 2 \mathrm{H}), 7.20-7.12(\mathrm{~m}, 4 \mathrm{H}), 7.04(\mathrm{~d}, J=1.8 \mathrm{~Hz}$, 2H), $3.18(\mathrm{~s}, 2 \mathrm{H}), 2.87(\mathrm{p}, J=6.9 \mathrm{~Hz}, 2 \mathrm{H}), 2.72(\mathrm{p}, J=6.7 \mathrm{~Hz}, 2 \mathrm{H}), 1.33(\mathrm{~d}, J=6.7 \mathrm{~Hz}, 6 \mathrm{H}), 1.26$ $-1.23(\mathrm{~m}, 12 \mathrm{H}), 1.21-1.16(\mathrm{~m}, 12 \mathrm{H}), 0.87(\mathrm{~d}, J=6.8 \mathrm{~Hz}, 6 \mathrm{H})$.

${ }^{13} \mathrm{C} \mathrm{NMR}\left(101 \mathrm{MHz}, \mathrm{CDCl}_{3}\right) \delta 149.0,148.4,148.1,133.0,132.3,132.1,130.6,128.1,127.2$, 125.8, 125.1, 123.5, 121.6, 120.1, 34.2, 30.9, 30.8, 27.3, 24.9, 24.1, 24.1, 24.0, 23.6.

1ㅏ P NMR $\left(202 \mathrm{MHz}, \mathrm{CDCl}_{3}\right) \delta 128.0$.

\section{C.2 Synthesis of Tetrachloro-Phthalimide Esters 1}

\section{C.2.1 Synthesis of $1 \mathrm{k}$}

Tetrachloro-phthalimide ester $\mathbf{1 k}$ was synthesized from N-hydroxytetrachlorophthalimide $\mathbf{S 1}{ }^{4}$ following reported procedures, ${ }^{5}$ as depicted in Scheme S1.
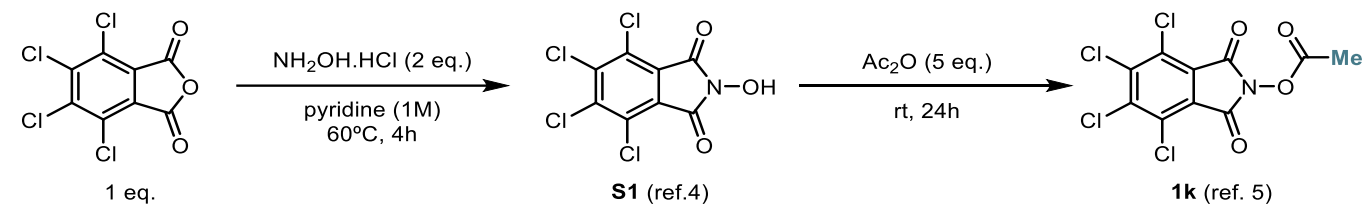

Scheme S1. Synthesis of S1 and 1k.

\section{C.2.2 General Procedure for the Synthesis of 1}

Tetrachloro-phthalimide esters $\mathbf{1 a - j}$, and $\mathbf{1} \mathbf{l}-\mathbf{s}$ were prepared from $\mathbf{S 1}$, using a typical procedure described below. Figure S2 depicts the general synthesis of 1, and the substrates that were previously reported. . $^{6,7,8,9,10}$

A round-bottom flask was charged with carboxylic acid (1.0 equiv), N-hydroxytetrachlorophthalimide S1 (1.0 equiv.) and DMAP (0.1 equiv.). Dichloromethane was added $(0.1 \mathrm{M})$, and the mixture was stirred vigorously. DIC (1.1 equiv.) was then added slowly, and the mixture was allowed to stir overnight, or until the acid was fully consumed as determined by TLC. The mixture was filtered over Celite and rinsed with additional $\mathrm{CH}_{2} \mathrm{Cl}_{2}$. The solvent was removed under reduced pressure, and purification by column chromatography (and recrystallization, if necessary) afforded the corresponding tetrachloro-phthalimide esters $\mathbf{1}$. 

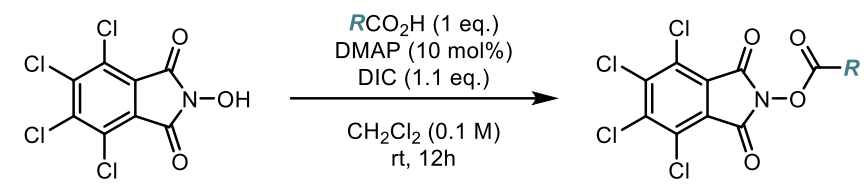

$\mathbf{s 1}$ (1 eq.)
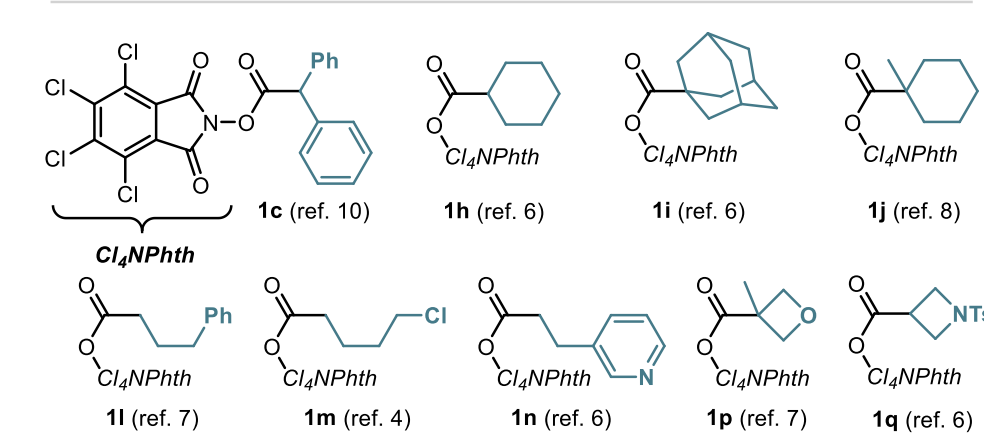

1h (ref. 6)

1 i (ref. 6)

1j (ref. 8)
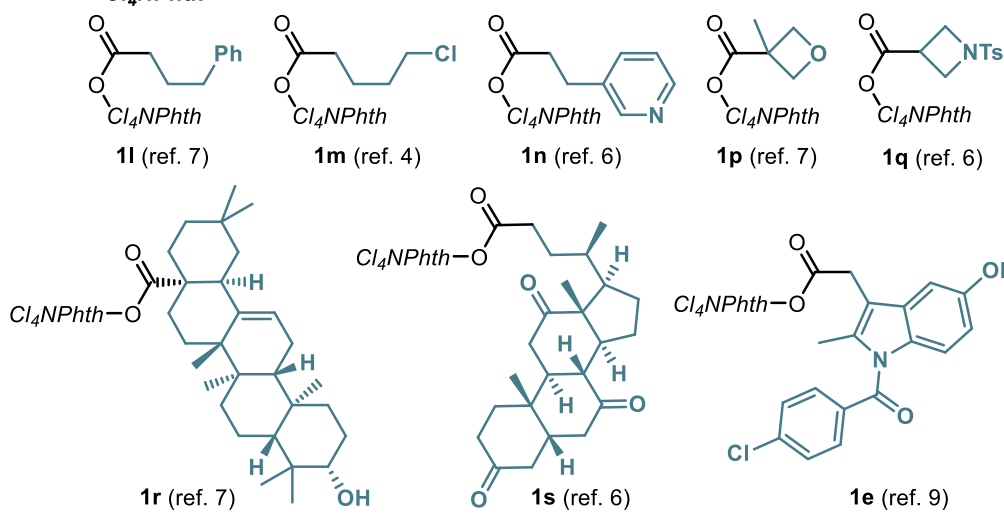

Figure S2. Previously reported tetrachloro-phthalimide esters 1.

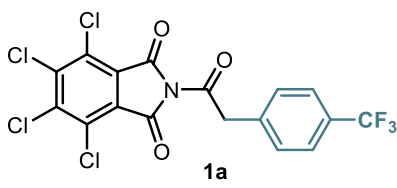

\section{4,5,6,7-tetrachloro-2-(2-(4- (trifluoromethyl)phenyl)acetyl)isoindoline-1,3-dione (1a)}

Prepared according to the general procedure using 4(Trifluoromethyl)phenylacetic acid $(612 \mathrm{mg}, 3.0 \mathrm{mmol})$. The crude mixture was purified by column chromatography $\left(\mathrm{SiO}_{2}, 95: 5\right.$ hexanes/EtOAc) to afford product 1a as a white solid (728 mg, $50 \%$ yield).

${ }^{1} \mathrm{H} \mathrm{NMR}\left(400 \mathrm{MHz}, \mathrm{CDCl}_{3}\right) \delta 7.66(\mathrm{~d}, J=8.1 \mathrm{~Hz}, 2 \mathrm{H}), 7.51(\mathrm{~d}, J=8.0 \mathrm{~Hz}, 2 \mathrm{H}), 4.06(\mathrm{~s}, 2 \mathrm{H})$. ${ }^{13} \mathrm{C} \mathrm{NMR}\left(101 \mathrm{MHz}, \mathrm{CDCl}_{3}\right) \delta 166.5,157.3,141.2,135.1,130.6,129.7,125.9(\mathrm{q}, J=3.8 \mathrm{~Hz})$, 124.6, 123.9 (q, $J=272.3 \mathrm{~Hz}), 37.4 .{ }^{19} \mathrm{~F} \mathrm{NMR}\left(376 \mathrm{MHz}, \mathrm{CDCl}_{3}\right) \delta-62.8$.

$\underline{\text { HRMS }}$ (ESI) Calculated for $\mathrm{C}_{18} \mathrm{H}_{10} \mathrm{Cl}_{4} \mathrm{~F}_{3} \mathrm{NNaO}_{5}[\mathrm{M}+\mathrm{MeOH}+\mathrm{Na}]^{+}:$539.9157, found: 539.9148 .

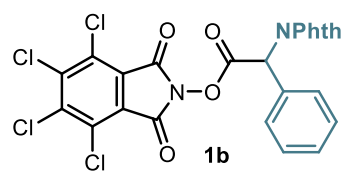

\section{4,5,6,7-tetrachloro-1,3-dioxoisoindolin-2-yl 2-(1,3-dioxoisoindolin- 2-yl)-2-phenylacetate (1b)}

Prepared according to the general procedure using 2-(1,3dioxoisoindolin-2-yl)-2-phenylacetic acid (1.40 g, $5.00 \mathrm{mmol})$. The crude mixture was purified by washing with cold acetone to afford product $\mathbf{1 b}$ as a white solid ( $1.80 \mathrm{~g}, 64 \%$ yield).

${ }^{1} \mathrm{H} \mathrm{NMR}\left(400 \mathrm{MHz}, \mathrm{CDCl}_{3}\right) \delta 7.89(\mathrm{dd}, J=5.5,3.0 \mathrm{~Hz}, 2 \mathrm{H}), 7.76-7.70(\mathrm{~m}, 4 \mathrm{H}), 7.46-7.38$ (m, 3H), 6.47 (s, 1H).

${ }^{13} \mathrm{C} \mathrm{NMR}\left(101 \mathrm{MHz}, \mathrm{CDCl}_{3}\right) \delta 166.3,164.8,134.5,132.4,131.6,129.9,129.4,128.9,128.6$, 123.9, 123.7, 54.0. HRMS (ESI) Calculated for $\mathrm{C}_{25} \mathrm{H}_{14} \mathrm{Cl}_{4} \mathrm{~N}_{2} \mathrm{NaO}_{7}[\mathrm{M}+\mathrm{MeOH}+\mathrm{Na}]^{+}$: 616.9447, found: 616.9444 . 


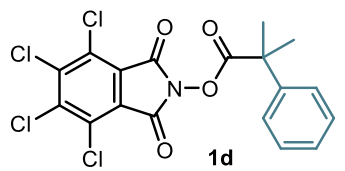

\section{4,5,6,7-tetrachloro-1,3-dioxoisoindolin-2-yl 2-methyl-2- phenylpropanoate (1d)}

Prepared according to the general procedure using 2-methyl-2phenylpropanoic acid (500 $\mathrm{mg}, 3.05 \mathrm{mmol}$ ). The crude mixture was purified by column chromatography $\left(\mathrm{SiO}_{2}, 98: 2\right.$ hexanes/EtOAc) to afford product $\mathbf{1 d}$ as a white solid (728 mg, 54\% yield).

${ }^{1} \mathrm{H} \mathrm{NMR}\left(400 \mathrm{MHz}, \mathrm{CDCl}_{3}\right) \delta 7.50-7.39(\mathrm{~m}, 4 \mathrm{H}), 7.36-7.30(\mathrm{~m}, 1 \mathrm{H}), 1.78(\mathrm{~s}, 6 \mathrm{H})$. ${ }^{13} \mathrm{C} \mathrm{NMR}\left(101 \mathrm{MHz}, \mathrm{CDCl}_{3}\right) \delta 157.6,130.4,128.8,127.5,125.7,124.8,46.3,26.8$. HRMS (ESI) Calculated for $\mathrm{C}_{19} \mathrm{H}_{15} \mathrm{Cl}_{4} \mathrm{NNaO}_{5}[\mathrm{M}+\mathrm{MeOH}+\mathrm{Na}]^{+}: 499.9597$, found: 499.9598 .

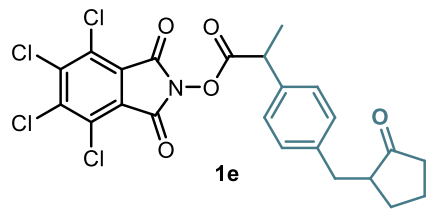

\section{4,5,6,7-tetrachloro-1,3-dioxoisoindolin-2-yl 2-(4-((2- oxocyclopentyl)methyl)phenyl)propanoate (1f)}

Prepared according to the general procedure using Loxoprofen (985 $\mathrm{mg}, 4.0 \mathrm{mmol}$ ). The crude mixture was purified by column chromatography ( $\mathrm{SiO}_{2}, 98: 2$ hexanes/EtOAc) to afford product $1 \mathrm{e}$ as a white solid $(1.22 \mathrm{~g}, 58 \%$ yield).

$\underline{{ }^{1} \mathrm{H} \mathrm{NMR}}\left(400 \mathrm{MHz}, \mathrm{CDCl}_{3}\right) \delta 7.34-7.30(\mathrm{~m}, 2 \mathrm{H}), 7.25-7.20(\mathrm{~m}, 2 \mathrm{H}), 4.12(\mathrm{q}, J=7.3 \mathrm{~Hz}, 1 \mathrm{H})$, $3.18(\mathrm{dd}, J=13.9,4.1 \mathrm{~Hz}, 1 \mathrm{H}), 2.56(\mathrm{ddd}, J=14.0,9.5,1.5 \mathrm{~Hz}, 1 \mathrm{H}), 2.43-2.32(\mathrm{~m}, 2 \mathrm{H}), 2.21-$ hb2.09 (m, 2H), 2.00 (dddd, $J=12.6,9.0,6.4,2.9 \mathrm{~Hz}, 1 \mathrm{H}), 1.85-1.72(\mathrm{~m}, 1 \mathrm{H}), 1.68(\mathrm{~d}, J=7.2$ $\mathrm{Hz}, 3 \mathrm{H}), 1.64-1.53(\mathrm{~m}, 1 \mathrm{H})$.

${ }^{13} \mathrm{C} \mathrm{NMR}\left(101 \mathrm{MHz}, \mathrm{CDCl}_{3}\right) \delta 170.4,141.0,139.8,135.8,130.5,129.5,127.6,124.7,50.9,42.5$, 38.2, 35.2, 29.2, 20.5, 18.9, 18.9 .

$\underline{\text { HRMS }}$ (ESI) Calculated for $\mathrm{C}_{24} \mathrm{H}_{21} \mathrm{Cl}_{4} \mathrm{NNaO}_{6}[\mathrm{M}+\mathrm{MeOH}+\mathrm{Na}]^{+}: 582.0015$, found: 582.0025 .

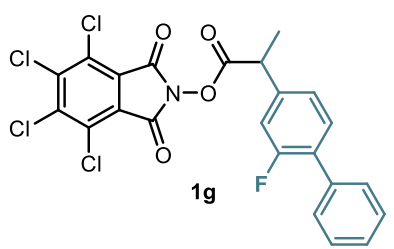

\section{4,5,6,7-tetrachloro-1,3-dioxoisoindolin-2-yl 2-(2-fluoro-[1,1'-} biphenyl]-4-yl)propanoate (1g)

Prepared according to the general procedure using Flurbiprofen (366 $\mathrm{mg}, 1.5 \mathrm{mmol})$. The crude mixture was purified by column chromatography ( $\mathrm{SiO}_{2}, 95: 5$ hexanes/EtOAc) to afford product $\mathbf{1 f}$ as a white solid (416 $\mathrm{mg}, 53 \%$ yield).

$\underline{{ }^{1} \mathrm{H} \mathrm{NMR}}\left(400 \mathrm{MHz}, \mathrm{CDCl}_{3}\right) \delta 7.58(\mathrm{dt}, J=8.2,1.5 \mathrm{~Hz}, 2 \mathrm{H}), 7.54-7.45(\mathrm{~m}, 3 \mathrm{H}), 7.44-7.38(\mathrm{~m}$, $1 \mathrm{H}), 7.31-7.20(\mathrm{~m}, 3 \mathrm{H}), 4.18(\mathrm{q}, J=7.0 \mathrm{~Hz}, 1 \mathrm{H}), 1.74(\mathrm{~d}, J=7.2 \mathrm{~Hz}, 3 \mathrm{H})$.

${ }^{13} \mathrm{C} \mathrm{NMR}\left(101 \mathrm{MHz}, \mathrm{CDCl}_{3}\right) \delta 169.9,141.1,139.0,138.9,135.2,131.3,131.3,130.5,129.0$, 129.0, 128.8, 128.5, 127.8, 124.7, 123.6, 123.5, 115.6, 115.3, 42.4, 18.8.

${ }^{19} \mathrm{~F} \mathrm{NMR}\left(376 \mathrm{MHz}, \mathrm{CDCl}_{3}\right) \delta-116.8$.

$\underline{\mathrm{HRMS}}$ (ESI) Calculated for $\mathrm{C}_{24} \mathrm{H}_{16} \mathrm{Cl}_{4} \mathrm{FNNaO}_{5}[\mathrm{M}+\mathrm{MeOH}+\mathrm{Na}]^{+}: 579.9659$, found: 579.9668 .

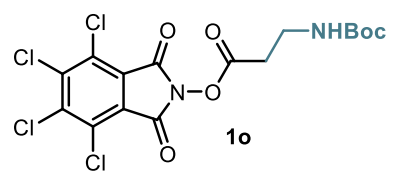

\section{4,5,6,7-tetrachloro-1,3-dioxoisoindolin-2-yl 3-((tert- butoxycarbonyl)amino)propanoate (10)}

Prepared according to the general procedure using NBoc-Alanine (568 $\mathrm{mg}, 3.00 \mathrm{mmol}$ ). The crude mixture was purified by washing with cold

acetone to afford product 10 as a yellow solid ( $841 \mathrm{~g}, 59 \%$ yield).

$\underline{{ }^{1} \mathrm{H} \mathrm{NMR}}\left(400 \mathrm{MHz}, \mathrm{CDCl}_{3}\right) \delta 5.06(\mathrm{~s}, 1 \mathrm{H}) .3 .55(\mathrm{q}, J=6.1 \mathrm{~Hz}, 2 \mathrm{H}), 2.92(\mathrm{t}, J=6.0 \mathrm{~Hz}, 2 \mathrm{H})$, $1.45(\mathrm{~s}, 9 \mathrm{H})$.

${ }^{13} \mathrm{C} \mathrm{NMR}\left(101 \mathrm{MHz}, \mathrm{CDCl}_{3}\right) \delta 157.4,155.8,141.1,130.6,124.6,36.1,32.0,28.4,28.3$. 
$\underline{\text { HRMS }}$ (ESI) Calculated for $\mathrm{C}_{17} \mathrm{H}_{18} \mathrm{Cl}_{4} \mathrm{~N}_{2} \mathrm{NaO}_{7}[\mathrm{M}+\mathrm{MeOH}+\mathrm{Na}]^{+}$: 524.9760, found: 524.9755.

\section{C.3 Synthesis of Pyridinium Salts 4}

Pyridinium salts 4 were synthesized following reported procedures (Figure S3). ${ }^{1112131415}$

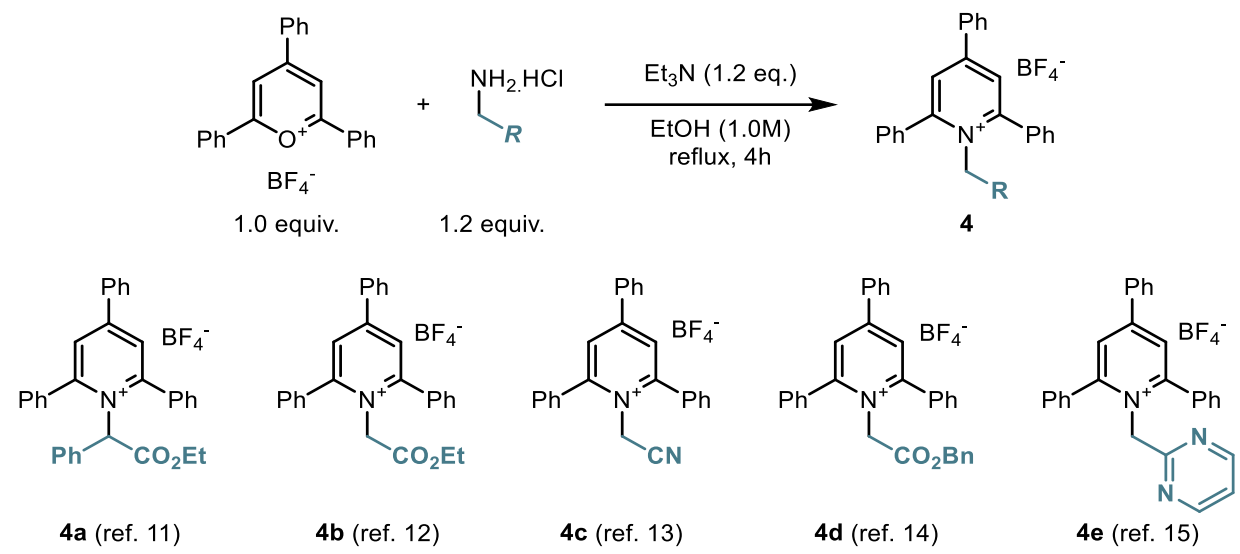

Figure S3. Pyridinium salts $\mathbf{4}$ synthetized according to reported procedures.

\section{C.4 Synthesis of Styrene Derivatives 5}

\section{C.4.1 General Procedure for the Wittig Reaction}

Alkenes $\mathbf{5 b}, \mathbf{5 h}-\mathbf{i}$, and $\mathbf{5 k}-\mathbf{l}$ were prepared from the corresponding ketones, using Wittig reactions as detailed below. The spectroscopic data of $\mathbf{5 b}, \mathbf{5 h}, \mathbf{5 i}$, and $\mathbf{5 k}$ were persistent with those reported previously (Figure S4). ${ }^{16,17,18,19}$

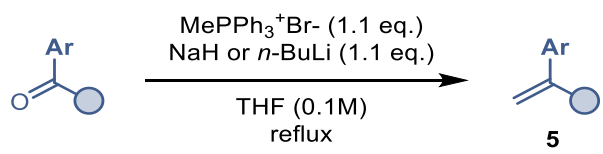

An oven-dried round-bottomed flask placed under an atmosphere of argon was charged with methyltriphenylphosphonium bromide $(1.1$ equiv) in dry THF $(0.1 \mathrm{M})$. The suspension was cooled down to $0^{\circ} \mathrm{C}$ and sodium hydride (1.1 equiv, $60 \%$ dispersion in mineral oil) or $n$ butyllithium (1.1 equiv., $2.5 \mathrm{M}$ in hexanes) was added slowly. The resulting mixture was stirred at $0^{\circ} \mathrm{C}$ for $30 \mathrm{~min}$ before adding dropwise a solution of the ketone (1.0 equiv) in dry THF. The reaction was stirred under reflux until complete conversion of the ketone was observed by TLC (typically overnight). The reaction was quenched by addition of water, and the organic phase extracted with ethyl acetate three times. The combined organic layers were washed with brine, dried over magnesium sulfate, filtered, and concentrated under reduced pressure to afford the crude compounds. The pure alkenes $\mathbf{5}$ were obtained by column chromatography on silica gel.

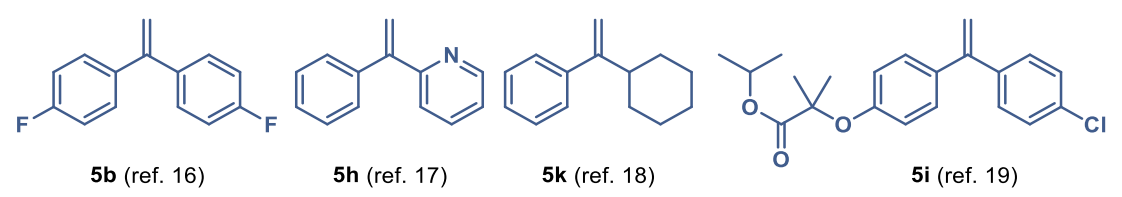

Figure S4. Previously reported alkenes 5 prepared using a Wittig reaction.

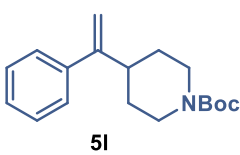

5 I

Tert-butyl 4-(1-phenylvinyl)piperidine-1-carboxylate (51)

Prepared according to the General Procedure using tert-butyl 4benzoylpiperidine-1-carboxylate $(579 \mathrm{mg}, 2.0 \mathrm{mmol})$, sodium hydride $(88.0$ 
$\mathrm{mg}, 2.2 \mathrm{mmol}$ ), and methyltriphenylphosphonium bromide (786 mg, $2.2 \mathrm{mmol}$ ) overnight. The crude mixture was purified by column chromatography $\left(\mathrm{SiO}_{2}, 90: 10\right.$ pentane/ $\left./ \mathrm{Et}_{2} \mathrm{O}\right)$ to afford product $\mathbf{5 1}$ as a colorless oil ( $246 \mathrm{mg}, 43 \%$ yield).

${ }^{1} \mathrm{H} \mathrm{NMR}\left(300 \mathrm{MHz}, \mathrm{CDCl}_{3}\right) \delta 7.38-7.29(\mathrm{~m}, 5 \mathrm{H}), 5.19(\mathrm{~d}, J=0.9 \mathrm{~Hz}, 1 \mathrm{H}), 5.03(\mathrm{t}, J=1.3 \mathrm{~Hz}$, $1 \mathrm{H}), 4.14(\mathrm{q}, J=7.1 \mathrm{~Hz}, 2 \mathrm{H}), 2.76(\mathrm{t}, J=12.8 \mathrm{~Hz}, 2 \mathrm{H}), 2.58(\mathrm{t}, J=11.8 \mathrm{~Hz}, 1 \mathrm{H}), 1.79(\mathrm{~d}, J=$ $13.4 \mathrm{~Hz}, 2 \mathrm{H}), 1.47$ (s, 9H), $1.44-1.33(\mathrm{~m}, 2 \mathrm{H})$.

${ }^{13} \mathrm{C} \mathrm{NMR}\left(75 \mathrm{MHz}, \mathrm{CDCl}_{3}\right) \delta 154.84,153.05,142.18,128.29,127.31,126.65,111.28,79.36$, 40.81, 31.46, 28.48 .

$\underline{\text { HRMS }}$ (ESI) Calculated for $\mathrm{C}_{18} \mathrm{H}_{25} \mathrm{NNaO}_{2}[\mathrm{M}+\mathrm{Na}]^{+}: 310.1777$, found: 310.1776 .

\section{C.4.2 Synthesis of Alkenes $5 \mathrm{c}-\mathrm{g}$}

Alkenes 5c-g were prepared following a reported procedure, ${ }^{20}$ as depicted in Figure S5.

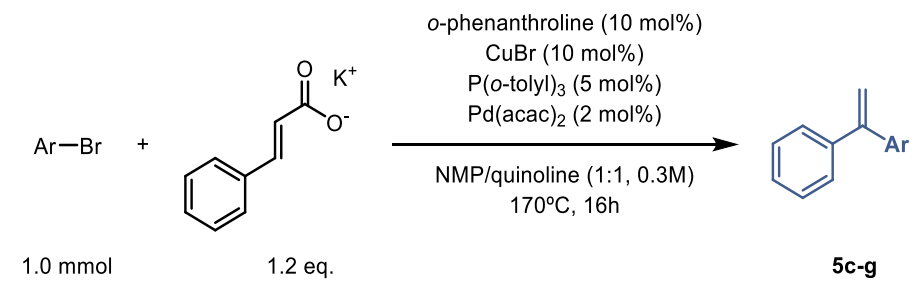<smiles>C=C(c1ccccc1)c1ccc2c(c1)OCCO2</smiles>

$5 c$<smiles>C=C(c1ccccc1)c1ccc(NC(C)(C)C)cc1</smiles>

5d

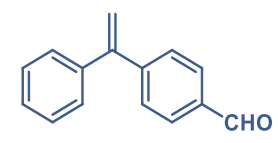

$5 e$<smiles>C=C(c1ccccc1)c1ccccc1CC</smiles>

$5 f$<smiles>C=C(c1ccccc1)c1cccs1</smiles>

$5 \mathrm{~g}$

Figure S5. Alkenes 5 synthetized according to a reported procedure.

\section{C.4.3 Synthesis of $2 \mathrm{~h}$}

$\mathbf{2 h}$ was synthesized adapting a procedure from the literature. ${ }^{21}$<smiles>NCCC1=CCCCC1</smiles><smiles>O=C1OC(=O)c2ccccc21</smiles>

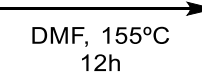
$12 \mathrm{~h}$ $1.5 \mathrm{eq}$<smiles>O=C1c2ccccc2C(=O)N1CCC1=CCCCC1</smiles>

2h: $86 \%$ yield

Phthalic anhydride (1.78 g, 1.5 equiv.) was added to a solution of 2-(1-Cyclohexenyl)ethylamine $(1.0 \mathrm{~mL}, 8.0 \mathrm{mmol}, 1.0$ equiv. $)$ in $\operatorname{DMF}(0.5 \mathrm{M})$ and refluxed at $155^{\circ} \mathrm{C}$ for 12 hours. The reaction was cooled to room temperature and poured into $50 \mathrm{~mL}$ of $1 \mathrm{M} \mathrm{HCl}$ and extracted with $\mathrm{Et}_{2} \mathrm{O}$. The crude mixture was purified by column chromatography ( $\mathrm{SiO} 2,90: 10$ pentane/Et $\mathrm{Et}_{2} \mathrm{O}$ ) to afford product $\mathbf{2 h}$ as white-off flakes ( $1.76 \mathrm{~g}, 86 \%$ yield).

${ }^{1} \mathrm{H}$ NMR $\left(500 \mathrm{MHz}, \mathrm{CDCl}_{3}\right) \delta 7.84(\mathrm{dd}, J=5.4,3.0 \mathrm{~Hz}, 2 \mathrm{H}), 7.73-7.68(\mathrm{~m}, 2 \mathrm{H}), 5.38(\mathrm{tdd}, J=$ 4.8, 2.6, $1.4 \mathrm{~Hz}, 1 \mathrm{H}), 3.79(\mathrm{dd}, J=7.5,6.7 \mathrm{~Hz}, 2 \mathrm{H}), 2.31(\mathrm{td}, J=7.0,1.3 \mathrm{~Hz}, 2 \mathrm{H}), 2.05$ (ddt, $J=$ 8.5, 6.0, 2.1 Hz, 2H), $1.88(\mathrm{ddt}, J=7.4,3.6,1.3 \mathrm{~Hz}, 2 \mathrm{H}), 1.68-1.60(\mathrm{~m}, 2 \mathrm{H}), 1.52(\mathrm{ddt}, J=8.7$, $6.2,2.8 \mathrm{~Hz}, 2 \mathrm{H})$. 
${ }^{13} \mathrm{C} \mathrm{NMR}\left(126 \mathrm{MHz}, \mathrm{CDCl}_{3}\right) \delta 168.27,134.25,133.78,132.19,123.94,123.07,36.70,36.62$, 27.91, 25.30, 22.85, 22.21.

\section{Photochemical Reactions}

\section{D.1 Experimental Set-up}

The photoreactor used in this study consisted of a $12.5 \mathrm{~cm}$ diameter jar fitted with 4 standard B29 size quickfit-glass joints arranged around a central B29 size joint (Figure S6). A commercial 1meter LED strip was wrapped around the jar, followed by a layer of aluminium foil and cotton for insulation.

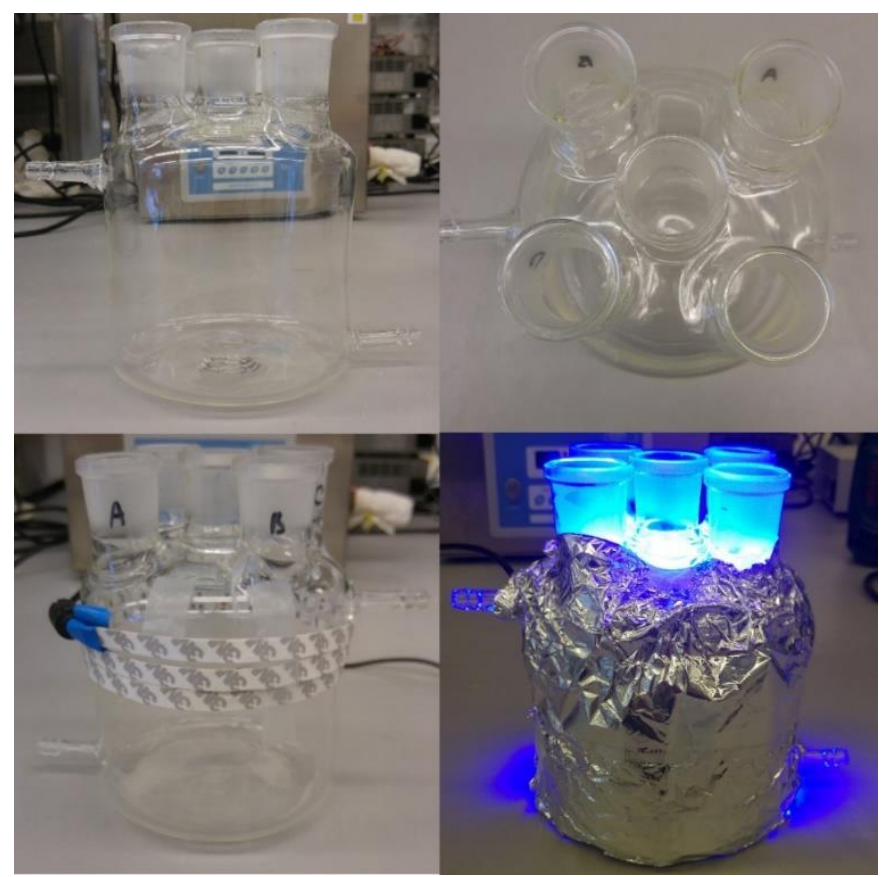

Figure S6. Photoreactor used in this study - pictures taken at different stages of the set-up assembly.

An inlet/outlet system provided circulation of liquid (ethylene glycol/water mixture) from a Huber Minichiller 300 inside the jar. This setup allowed the performance of reactions at temperatures ranging from $-20^{\circ} \mathrm{C}$ to $80^{\circ} \mathrm{C}$ with accurate control of the reaction temperature $\left( \pm 1^{\circ} \mathrm{C}\right.$, Figure S7). 

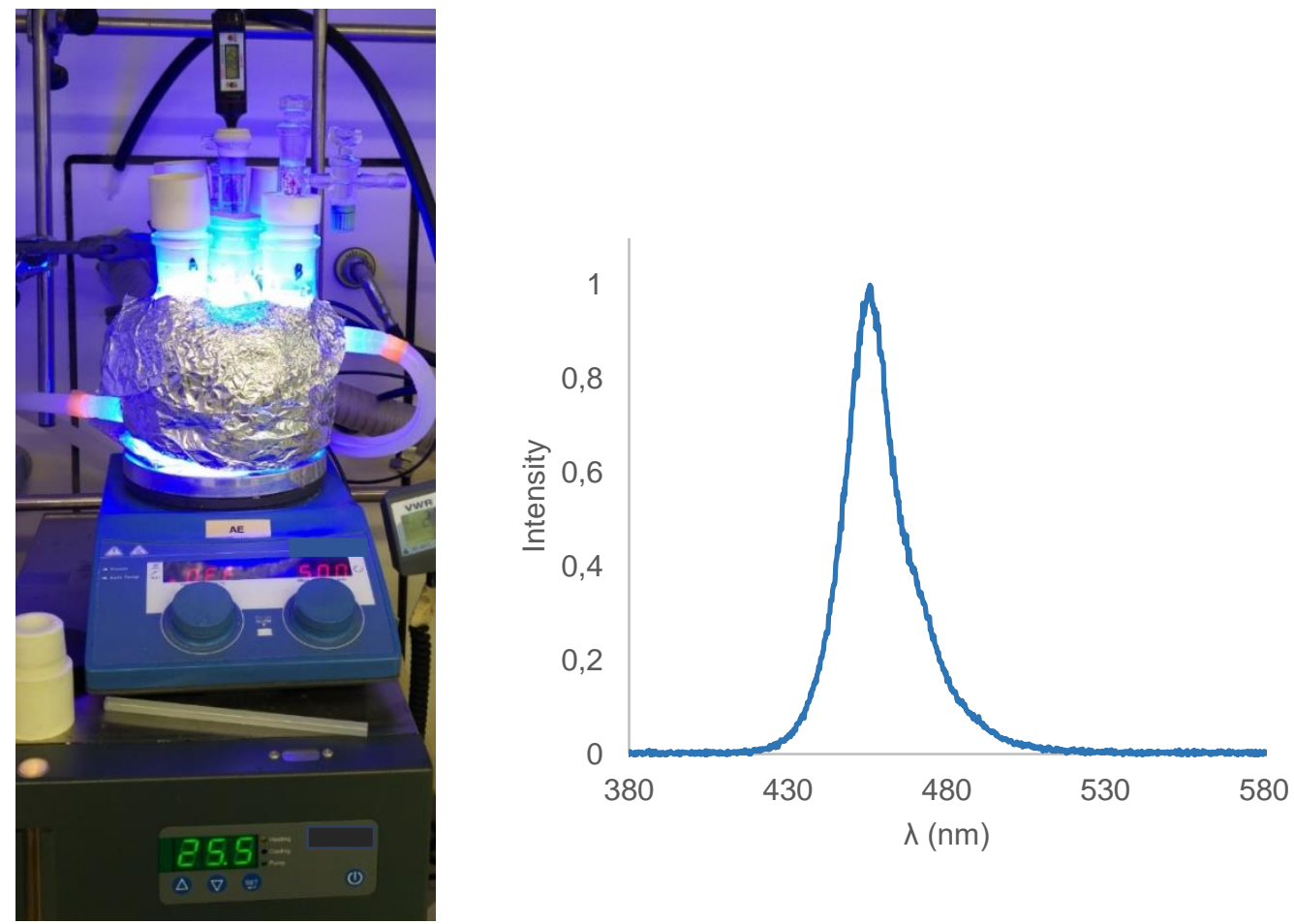

Figure S7. Fully assembled temperature-controlled photoreactor in operation (left). Emission spectrum of the $465 \mathrm{~nm}$ LED strip used in this reactor (right).

In order to maintain consistent illumination between different experiments, only the four external positions were used to perform reactions. The central position was used to monitor the temperature using a thermometer inside an inserted Schlenk tube, ensuring that the reaction mixtures were at the desired temperature.

\section{D.2 Direct C-H Allylic Benzylation}

\section{D.2.1 General Procedure A}

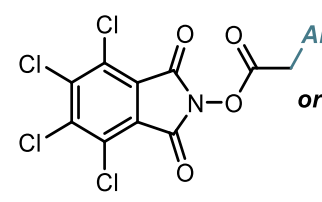

1

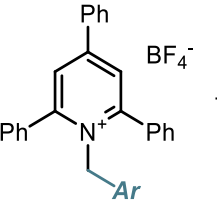

4<smiles>[R]C1=CCC[N]1</smiles>

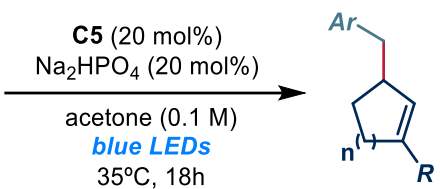

To an argon-purged glass vial, containing the dithiophosphoric acid catalyst $\mathbf{C 5}$ ( 0.2 equiv.), tetrachloro-phthalimide ester 1 or pyridinium salt 4 ( 1 equiv.), and $\mathrm{Na}_{2} \mathrm{HPO}_{4}(0.2$ equiv.), was added the allylic precursor 2 (20 equiv.) followed by argon-sparged HPLC grade acetone (0.1M). The vial was sealed with Parafilm, and placed in the irradiation setup, maintained at a temperature of $35^{\circ} \mathrm{C}$. The reaction was stirred for $18 \mathrm{~h}$, then the solvent was evaporated and the crude mixture purified by flash column chromatography on silica gel to furnish the product 3 . 


\section{D.2.2 Characterization of Products 3}

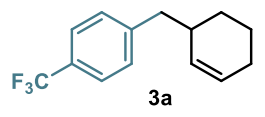

\section{1-(cyclohex-2-en-1-ylmethyl)-4-(trifluoromethyl)benzene (3a)}

Prepared according to General Procedure A using the radical precursor 1a (97.4 $\mathrm{mg}, 0.2 \mathrm{mmol})$ and cyclohexene $2 \mathrm{a}(406 \mu \mathrm{L}, 4.0 \mathrm{mmol})$. The crude mixture was purified by column chromatography $\left(\mathrm{SiO}_{2}, 100 \%\right.$ pentane) to afford product $\mathbf{3 a}$ as a colorless oil (19.8 $\mathrm{mg}, 41 \%$ yield, average of two runs).

${ }^{1} \mathrm{H} \mathrm{NMR}\left(400 \mathrm{MHz}, \mathrm{CDCl}_{3}\right) \delta 7.53(\mathrm{~d}, J=8.0 \mathrm{~Hz}, 2 \mathrm{H}), 7.28(\mathrm{~d}, J=8.0 \mathrm{~Hz}, 2 \mathrm{H}), 5.71(\mathrm{dtd}, J=$ 9.8, 3.5, $2.2 \mathrm{~Hz}, 1 \mathrm{H}), 5.53(\mathrm{dq}, J=10.1,2.4 \mathrm{~Hz}, 1 \mathrm{H}), 2.69$ (dd, $J=13.3,7.2 \mathrm{~Hz}, 1 \mathrm{H}), 2.60$ (dd, $J$ $=13.3,8.0 \mathrm{~Hz}, 1 \mathrm{H}), 2.39$ (ddtq, $J=10.4,7.6,5.0,2.7 \mathrm{~Hz}, 1 \mathrm{H}), 1.99(\mathrm{dtt}, J=7.4,5.2,2.7 \mathrm{~Hz}$, 2H), 1.71 (tdt, $J=12.6,8.7,4.3 \mathrm{~Hz}, 2 \mathrm{H}), 1.60-1.48(\mathrm{~m}, 1 \mathrm{H}), 1.40-1.26(\mathrm{~m}, 1 \mathrm{H})$.

${ }^{13} \mathrm{C} \mathrm{NMR}\left(101 \mathrm{MHz}, \mathrm{CDCl}_{3}\right) \delta 145.0,130.6,129.4,129.1(\mathrm{q}, J=275.1 \mathrm{~Hz}), 127.9,125.1(\mathrm{q}, J=$ $3.8 \mathrm{~Hz}), 42.5,40.1,37.0,28.8,26.0,25.4,25.3,22.2,21.2$.

${ }^{19} \mathrm{~F} \mathrm{NMR}\left(376 \mathrm{MHz}, \mathrm{CDCl}_{3}\right) \delta-62.4$.

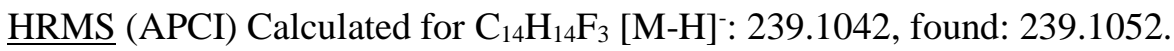

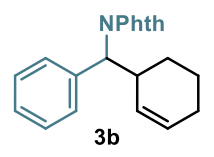

\section{2-(cyclohex-2-en-1-yl(phenyl)methyl)isoindoline-1,3-dione (3b)}

Prepared according to General Procedure A using the radical precursor $\mathbf{1 b}$ (56.4 $\mathrm{mg}, 0.1 \mathrm{mmol})$, and cyclohexene $2 \mathbf{a}(203 \mu \mathrm{L}, 2.0 \mathrm{mmol})$. The crude mixture was purified by column chromatography $\left(\mathrm{SiO}_{2}, 20: 1\right.$ Hexane/EtOAc $)$ to afford product $\mathbf{3 b}$ as a white solid (7.9 mg, 25\% yield, 1.1:1 dr., average of two runs).

${ }^{1} \mathrm{H}$ NMR $\left(500 \mathrm{MHz}, \mathrm{CDCl}_{3}\right)$ 1.1:1 mixture of diastereoiomers $\delta 7.79$ (ddd, $J=5.4,3.8,3.0 \mathrm{~Hz}$, $2 \mathrm{H}), 7.67(\mathrm{dd}, J=5.5,3.0 \mathrm{~Hz}, 2 \mathrm{H}), 7.63(\mathrm{tt}, J=8.1,1.3 \mathrm{~Hz}, 2 \mathrm{H}), 7.37-7.30(\mathrm{~m}, 2 \mathrm{H}), 7.29-7.24$ $(\mathrm{m}, 1 \mathrm{H}), 5.78(\mathrm{dtd}, J=9.8,3.7,2.0 \mathrm{~Hz}, 0.5 \mathrm{H}), 5.71(\mathrm{dtd}, J=9.9,3.7,2.2 \mathrm{~Hz}, 0.5 \mathrm{H}), 5.56-5.50$ $(\mathrm{m}, 0.5 \mathrm{H}), 5.33-5.26(\mathrm{~m}, 0.5 \mathrm{H}), 5.00(\mathrm{~d}, J=12.3 \mathrm{~Hz}, 0.5 \mathrm{H}), 4.98(\mathrm{~d}, J=12.1 \mathrm{~Hz}, 0.5 \mathrm{H}), 3.83-$ $3.71(\mathrm{~m}, 0.5 \mathrm{H}), 2.05-1.99(\mathrm{~m}, 2 \mathrm{H}), 1.83-1.68(\mathrm{~m}, 0.5 \mathrm{H}), 1.68-1.48(\mathrm{~m}, 0.5 \mathrm{H}), 1.37-1.27$ (m, $0.5 \mathrm{H}), 1.16$ (dddd, $J=12.9,10.4,7.9,2.9 \mathrm{~Hz}, 0.5 \mathrm{H})$.

${ }^{13} \mathrm{C} \mathrm{NMR}\left(126 \mathrm{MHz}, \mathrm{CDCl}_{3}\right)$ 1.1:1 mixture of diastereoiomers $\delta 168.4,168.4,138.8,138.4$, 133.9, 131.8, 131.8, 129.9, 129.4, 129.1, 129.1, 128.6, 128.0, 127.9, 127.5, 127.0, 127.0, 123.2, 123.2, 60.6, 60.0, 34.7, 34.1, 27.1, 26.6, 25.3, 25.2, 20.7, 20.5.

HRMS (ESI) Calculated for $\mathrm{C}_{21} \mathrm{H}_{19} \mathrm{NNaO}_{2}[\mathrm{M}+\mathrm{Na}]^{+}: 340.1308$, found: 340.1304

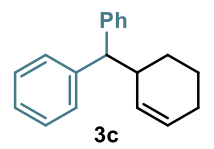

\section{(cyclohex-2-en-1-ylmethylene)dibenzene (3c)}

Prepared according to General Procedure A using the radical precursor 1c (49.5 $\mathrm{mg}, 0.1 \mathrm{mmol})$, and cyclohexene $\mathbf{2 a}(203 \mu \mathrm{L}, 2.0 \mathrm{mmol})$. The crude mixture was purified by column chromatography $\left(\mathrm{SiO}_{2}, 99: 1\right.$ pentane $\left./ \mathrm{CH}_{2} \mathrm{Cl}_{2}\right)$ to afford product $3 \mathbf{c}$ as a white solid (12.4 mg, 50\% yield, average of two runs).

${ }^{1} \mathrm{H}$ NMR $\left(400 \mathrm{MHz}, \mathrm{CDCl}_{3}\right) \delta 7.34-7.29(\mathrm{~m}, 2 \mathrm{H}), 7.29-7.22(\mathrm{~m}, 6 \mathrm{H}), 7.17-7.10(\mathrm{~m}, 2 \mathrm{H})$, $5.66(\mathrm{dtd}, J=9.9,3.5,2.0 \mathrm{~Hz}, 1 \mathrm{H}), 5.45(\mathrm{dqd}, J=10.3,2.2,1.0 \mathrm{~Hz}, 1 \mathrm{H}), 3.59(\mathrm{~d}, J=11.4 \mathrm{~Hz}$, 1H), $2.98(\mathrm{dddp}, J=10.9,8.0,5.4,2.7 \mathrm{~Hz}, 1 \mathrm{H}), 1.98(\mathrm{qt}, J=5.1,3.1 \mathrm{~Hz}, 2 \mathrm{H}), 1.76-1.66(\mathrm{~m}$, $1 \mathrm{H}), 1.65-1.56(\mathrm{~m}, 1 \mathrm{H}), 1.56-1.44(\mathrm{~m}, 1 \mathrm{H}), 1.19(\mathrm{dddd}, J=12.8,11.2,8.6,2.8 \mathrm{~Hz}, 1 \mathrm{H})$. 
${ }^{13} \mathrm{C}$ NMR $\left(101 \mathrm{MHz}, \mathrm{CDCl}_{3}\right) \delta 144.2,143.9,130.0,128.5,128.4,128.3,128.2,128.0,126.1$, $126.1,58.1,38.9,28.3,25.4,21.5$. Analytical data are in agreement with the one reported in literature. $^{22}$

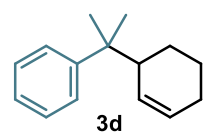

\section{(2-(cyclohex-2-en-1-yl)propan-2-yl)benzene (3d)}

Prepared according to General Procedure A using the radical precursor 1d (89.4 $\mathrm{mg}, 0.2 \mathrm{mmol})$, and cyclohexene $2 \mathbf{a}(406 \mu \mathrm{L}, 4.0 \mathrm{mmol})$. The crude mixture was purified by column chromatography $\left(\mathrm{SiO}_{2}, 100 \%\right.$ pentane) to afford product $\mathbf{3 d}$ as a colorless oil (18.0 mg, 45\% yield, average of two runs).

${ }^{1} \mathrm{H} \mathrm{NMR}\left(500 \mathrm{MHz}, \mathrm{CDCl}_{3}\right) \delta 7.37-7.34(\mathrm{~m}, 2 \mathrm{H}), 7.33-7.28(\mathrm{~m}, 2 \mathrm{H}), 7.20-7.16(\mathrm{~m}, 1 \mathrm{H})$, $5.73-5.66(\mathrm{~m}, 1 \mathrm{H}), 5.49(\mathrm{dp}, J=10.3,2.0 \mathrm{~Hz}, 1 \mathrm{H}), 2.46$ (dddt, $J=10.2,8.0,5.2,2.6 \mathrm{~Hz}, 1 \mathrm{H})$, 1.92 (dddd, $J=9.0,4.4,3.3,2.0 \mathrm{~Hz}, 2 \mathrm{H}), 1.77-1.69(\mathrm{~m}, 1 \mathrm{H}), 1.50-1.40(\mathrm{~m}, 1 \mathrm{H}), 1.30(\mathrm{~s}, 3 \mathrm{H})$, $1.26(\mathrm{~s}, 3 \mathrm{H}), 1.20$ (tdd, $J=12.7,10.7,2.9 \mathrm{~Hz}, 2 \mathrm{H})$.

${ }^{13} \mathrm{C} \mathrm{NMR}\left(126 \mathrm{MHz}, \mathrm{CDCl}_{3}\right) \delta 149.8,129.0,128.4,127.9,126.2,125.4,46.5,40.2,30.329 .7$, 25.3, 25.2, 25.0, 24.7, 22.8 .

$\underline{\text { HRMS }}$ (APCI) Calculated for $\mathrm{C}_{15} \mathrm{H}_{21}[\mathrm{M}+\mathrm{H}]^{+}:$201.1638, found: 201.1633 .

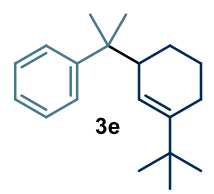

\section{(2-(3-(tert-butyl)cyclohex-2-en-1-yl)propan-2-yl)benzene (3e)}

Prepared according to General Procedure A using the radical precursor 1d (49.7 $\mathrm{mg}, 0.1 \mathrm{mmol})$, and 1-tert-Butyl-1-cyclohexene $2 \mathbf{b}(170 \mu \mathrm{L}, 1.0 \mathrm{mmol})$. The crude mixture was purified by column chromatography $\left(\mathrm{SiO}_{2}, 100 \%\right.$ pentane) to afford product $3 \mathbf{e}$ as a colorless oil (10 $\mathrm{mg}$, single regioisomer, 39\% yield, average of two runs).

${ }^{1} \mathrm{H}$ NMR $\left(400 \mathrm{MHz}, \mathrm{CDCl}_{3}\right) \delta 7.40-7.29(\mathrm{~m}, 4 \mathrm{H}), 7.22-7.17(\mathrm{~m}, 1 \mathrm{H}), 5.33(\mathrm{tt}, J=2.2,0.9 \mathrm{~Hz}$, $1 \mathrm{H}), 2.43$ (dddd, $J=10.5,5.9,4.0,2.2 \mathrm{~Hz}, 1 \mathrm{H}), 2.06-1.98(\mathrm{~m}, 1 \mathrm{H}), 1.91-1.82(\mathrm{~m}, 1 \mathrm{H}), 1.82-$ $1.73(\mathrm{~m}, 1 \mathrm{H}), 1.54-1.46(\mathrm{~m}, 1 \mathrm{H}), 1.41-1.33(\mathrm{~m}, 1 \mathrm{H}), 1.31(\mathrm{~s}, 3 \mathrm{H}), 1.27$ (s, 3H), 1.09 (ddd, $J=$ $12.6,10.6,3.2 \mathrm{~Hz}, 1 \mathrm{H}), 1.00(\mathrm{~s}, 9 \mathrm{H})$.

${ }^{13} \mathrm{C}$ NMR $\left(101 \mathrm{MHz}, \mathrm{CDCl}_{3}\right) \delta 150.0,147.0,127.8,126.3,125.3,119.2,46.7,40.7,35.5,29.1$, 25.3, 25.0, 24.8, 24.6, 23.5.

$\underline{\text { HRMS }}$ (APCI) Calculated for $\mathrm{C}_{19} \mathrm{H}_{29}[\mathrm{M}+\mathrm{H}]^{+}:$257.2264, found: 257.2256.

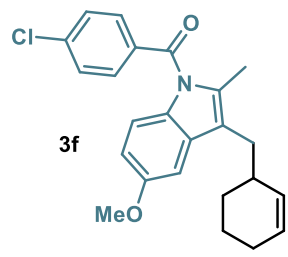

\section{(4-chlorophenyl)(3-(cyclohex-2-en-1-ylmethyl)-5-methoxy-2-methyl- 1H-indol-1-yl)methanone (3f)}

Prepared according to General Procedure A using the radical precursor 1e (64.1 mg, $0.1 \mathrm{mmol})$, and cyclohexene $2 \mathbf{a}(203 \mu \mathrm{L}, 2.0 \mathrm{mmol})$. The crude mixture was purified by column chromatography $\left(\mathrm{SiO}_{2}, \quad 98: 2\right.$ hexanes/EtOAc) to afford product $\mathbf{3 f}$ as a yellowish oil (12.6 mg, 32\% yield, average of two runs). ${ }^{1} \mathrm{H} \mathrm{NMR}\left(500 \mathrm{MHz}, \mathrm{CDCl}_{3}\right) \delta 7.68-7.64(\mathrm{~m}, 2 \mathrm{H}), 7.52-7.47(\mathrm{~m}, 2 \mathrm{H}), 6.96-6.86(\mathrm{~m}, 2 \mathrm{H})$, $6.67(\mathrm{dd}, J=9.0,2.6 \mathrm{~Hz}, 1 \mathrm{H}), 5.73(\mathrm{td}, J=6.0,2.9 \mathrm{~Hz}, 1 \mathrm{H}), 5.63(\mathrm{dd}, J=10.1,2.3 \mathrm{~Hz}, 1 \mathrm{H}), 3.85$ (s, 3H), $2.68(\mathrm{dd}, J=14.0,7.3 \mathrm{~Hz}, 1 \mathrm{H}), 2.60(\mathrm{dd}, J=14.0,8.2 \mathrm{~Hz}, 1 \mathrm{H}), 2.47(\mathrm{~s}, 1 \mathrm{H}), 2.34(\mathrm{~s}, 3 \mathrm{H})$, $2.03(\mathrm{~d}, J=5.7 \mathrm{~Hz}, 2 \mathrm{H}), 1.79(\mathrm{dq}, J=10.1,6.2,5.4 \mathrm{~Hz}, 2 \mathrm{H}), 1.55(\mathrm{~s}, 1 \mathrm{H}), 1.34(\mathrm{td}, J=10.9,8.5$ $\mathrm{Hz}, 1 \mathrm{H})$.

${ }^{13} \mathrm{C} \mathrm{NMR}\left(126 \mathrm{MHz}, \mathrm{CDCl}_{3}\right) \delta 206.9,168.3,155.8,139.0,134.7,134.3,131.6,131.3,131.1$, 131.0, 129.1, 127.6, 118.6, 114.9, 110.8, 101.9, 55.8, 35.8, 30.9, 30.7, 29.3, 25.4, 21.3, 13.6.

HRMS (ESI) Calculated for $\mathrm{C}_{24} \mathrm{H}_{24} \mathrm{ClNNaO}_{2}[\mathrm{M}+\mathrm{Na}]^{+}: 416.1388$, found: 416.1399 . 


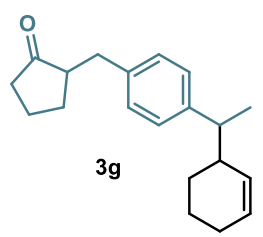

2-(4-(1-(cyclohex-2-en-1-yl)ethyl)benzyl)cyclopentan-1-one (3g)

Prepared according to General Procedure A using the radical precursor $1 \mathbf{f}$ (52.9 $\mathrm{mg}, 0.1 \mathrm{mmol})$, and cyclohexene $2 \mathbf{a}(203 \mu \mathrm{L}, 2.0 \mathrm{mmol})$. The crude mixture was purified by column chromatography $\left(\mathrm{SiO}_{2}, 98: 2\right.$ hexanes/EtOAc) to afford product $3 \mathrm{~g}$ as a colorless oil $(12.4 \mathrm{mg}, 44 \%$ yield, $1.1: 1 \mathrm{dr}$, average of two runs).

$\underline{{ }^{1} \mathrm{H} \mathrm{NMR}}\left(400 \mathrm{MHz}, \mathrm{CDCl}_{3}\right)$ 1.1:1 mixture of diastereoiomers $\delta 7.11(\mathrm{~s}, 4 \mathrm{H}), 5.84-5.72(\mathrm{~m}, 1 \mathrm{H})$, $5.64(\mathrm{dq}, J=10.1,3.4 \mathrm{~Hz}, 0.5 \mathrm{H}), 5.38(\mathrm{dd}, J=10.2,2.3 \mathrm{~Hz}, 0.5 \mathrm{H}), 3.14(\mathrm{dt}, J=13.9,3.8 \mathrm{~Hz}$, $1 \mathrm{H}), 2.68-2.48(\mathrm{~m}, 2 \mathrm{H}), 2.43-2.32(\mathrm{~m}, 2 \mathrm{H}), 2.30-2.21(\mathrm{~m}, 1 \mathrm{H}), 2.19-2.06(\mathrm{~m}, 2 \mathrm{H}), 1.97$ $(\mathrm{dtt}, J=8.4,6.1,3.3 \mathrm{~Hz}, 3 \mathrm{H}), 1.87-1.65(\mathrm{~m}, 2 \mathrm{H}), 1.64-1.54(\mathrm{~m}, 1 \mathrm{H}), 1.53-1.39(\mathrm{~m}, 1 \mathrm{H}), 1.33$ $-1.27(\mathrm{~m}, 2 \mathrm{H}), 1.27-1.22(\mathrm{~m}, 2 \mathrm{H}), 1.21-1.11(\mathrm{~m}, 0.5 \mathrm{H}), 0.97-0.78(\mathrm{~m}, 0.5 \mathrm{H})$.

${ }^{13} \mathrm{C}$ NMR $\left(101 \mathrm{MHz}, \mathrm{CDCl}_{3}\right)$ 1.1:1 mixture of diastereoiomers $\delta$ 144.5, 144.3, 137.4, 130.8, 129.6, 128.8, 128.6, 128.2, 127.9, 127.8, 127.5, 127.5, 51.1, 44.6, 44.2, 41.9, 41.9, 41.8, 38.3, $35.2,35.2,29.3,27.6,26.5,25.4,25.3,22.0,21.4,20.6,18.7,18.7,18.5,15.6,-18.5$.

HRMS (APCI) Calculated for $\mathrm{C}_{20} \mathrm{H}_{27} \mathrm{O}[\mathrm{M}+\mathrm{H}]^{+}: 283.2056$, found: 283.2051.

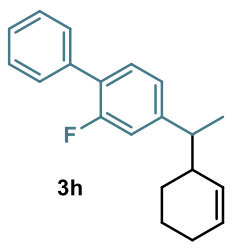

4-(1-(cyclohex-2-en-1-yl)ethyl)-2-fluoro-1,1'-biphenyl (3h)

Prepared according to General Procedure A using the radical precursor 1g (52.7 $\mathrm{mg}, 0.1 \mathrm{mmol})$, and cyclohexene $\mathbf{2 a}(203 \mu \mathrm{L}, 2.0 \mathrm{mmol})$. The crude mixture was purified by column chromatography $\left(\mathrm{SiO}_{2}\right.$, hexanes) to afford product $\mathbf{3 h}$ as a colorless oil ( $13.8 \mathrm{mg}, 49 \%$ yield, 1.1:1 dr, average of two runs).

${ }^{1} \mathrm{H} \mathrm{NMR}\left(400 \mathrm{MHz}, \mathrm{CDCl}_{3}\right) 1.1: 1$ mixture of diastereoiomers $\delta 7.61-7.55(\mathrm{~m}, 2 \mathrm{H}), 7.50-7.44$ (m, 2H), $7.41-7.35(\mathrm{~m}, 2 \mathrm{H}), 7.09-6.98(\mathrm{~m}, 2 \mathrm{H}), 5.87-5.77(\mathrm{~m}, 1 \mathrm{H}), 5.75-5.67(\mathrm{~m}, 0.5 \mathrm{H})$, 5.46 (ddd, $J=10.2,2.3,1.2 \mathrm{~Hz}, 0.5 \mathrm{H}$ ), 2.67 (ddt, $J=14.5,11.3,7.4 \mathrm{~Hz}, 1 \mathrm{H}$ ), 2.34 (ddddd, $J=$ $18.5,11.5,8.1,5.3,2.8 \mathrm{~Hz}, 1 \mathrm{H}), 2.00(\mathrm{ddt}, J=6.0,4.0,2.1 \mathrm{~Hz}, 2 \mathrm{H}), 1.91-1.78(\mathrm{~m}, 1 \mathrm{H}), 1.78-$ $1.68(\mathrm{~m}, 1 \mathrm{H}), 1.62-1.46(\mathrm{~m}, 1 \mathrm{H}), 1.37-1.28(\mathrm{~m}, 3 \mathrm{H}), 1.28-1.20(\mathrm{~m}, 0.5 \mathrm{H}), 0.96-0.81(\mathrm{~m}$, $0.5 \mathrm{H})$.

${ }^{13} \mathrm{C}$ NMR $\left(101 \mathrm{MHz}, \mathrm{CDCl}_{3}\right)$ 1.1:1 mixture of diastereoiomers $\delta 160.9,158.4,148.5,135.9$, 130.3, 130.3, 130.2, 129.2, 129.0, 128.9, 128.6, 128.4, 128.0, 127.4, 126.3, 123.8, 123.6, 115.3, $115.1,114.8,44.6,44.3,41.8,41.7,27.6,26.5,25.4,25.3,21.9,21.4,18.6,18.4$.

${ }^{19} \mathrm{~F}$ NMR $\left(376 \mathrm{MHz}, \mathrm{CDCl}_{3}\right) \delta-118.8$.

HRMS (APCI) Calculated for $\mathrm{C}_{20} \mathrm{H}_{22} \mathrm{~F}[\mathrm{M}+\mathrm{H}]^{+}:$281.1700, found: 281.1701 .

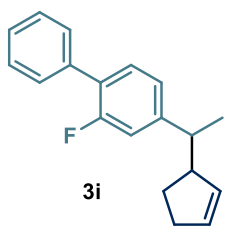

\section{4-(1-(cyclopent-2-en-1-yl)ethyl)-2-fluoro-1,1'-biphenyl (3i)}

Prepared according to General Procedure A using the radical precursor 1g (52.7 $\mathrm{mg}, 0.1 \mathrm{mmol})$ and cyclopentene $2 \mathbf{c}(176 \mu \mathrm{L}, 2.0 \mathrm{mmol})$. The crude mixture was purified by column chromatography $\left(\mathrm{SiO}_{2}, 30: 1\right.$ hexanes $\left./ \mathrm{CH}_{2} \mathrm{Cl}_{2}\right)$ to afford product $3 \mathbf{i}$ as a colorless oil ( $12.1 \mathrm{mg}, 45 \%$ yield, $1.1: 1 \mathrm{dr}$, average of two runs).

${ }^{1} \mathrm{H}$ NMR $\left(500 \mathrm{MHz}, \mathrm{CDCl}_{3}\right)$ 1.1:1 mixture of diastereoisomers $\delta 7.56(\mathrm{dt}, J=7.9,1.5 \mathrm{~Hz}, 2 \mathrm{H})$, $7.48-7.40(\mathrm{~m}, 2 \mathrm{H}), 7.39-7.32(\mathrm{~m}, 2 \mathrm{H}), 7.04(\mathrm{dd}, J=7.9,1.7 \mathrm{~Hz}, 1 \mathrm{H}), 7.00(\mathrm{dt}, J=12.1,1.8$ $\mathrm{Hz}, 1 \mathrm{H}), 5.87-5.80(\mathrm{~m}, 1 \mathrm{H}), 5.73(\mathrm{dq}, J=5.8,2.3 \mathrm{~Hz}, 0.5 \mathrm{H}), 5.45(\mathrm{dq}, J=6.0,2.1 \mathrm{~Hz}, 0.5 \mathrm{H})$, $2.95-2.84(\mathrm{~m}, 1 \mathrm{H}), 2.64-2.54(\mathrm{~m}, 1 \mathrm{H}), 2.35-2.20(\mathrm{~m}, 2 \mathrm{H}), 2.10(\mathrm{dtd}, J=12.7,8.1,4.5 \mathrm{~Hz}$, $0.5 \mathrm{H}), 1.85(\mathrm{dtd}, J=13.4,8.6,5.0 \mathrm{~Hz}, 0.5 \mathrm{H}), 1.62-1.53(\mathrm{~m}, 0.5 \mathrm{H}), 1.45(\mathrm{ddt}, J=12.9,9.0,6.3$ $\mathrm{Hz}, 0.5 \mathrm{H}), 1.31(\mathrm{~d}, J=6.9 \mathrm{~Hz}, 1.5 \mathrm{H}), 1.28(\mathrm{~d}, J=7.0 \mathrm{~Hz}, 1.5 \mathrm{H}), 1.29-1.26(\mathrm{~m}, 1 \mathrm{H})$. 
${ }^{13} \mathrm{C} \mathrm{NMR}\left(126 \mathrm{MHz}, \mathrm{CDCl}_{3}\right)$ 1.1:1 mixture of diastereoisomers $\delta 160.6,158.7,148.8(\mathrm{~d}, J=7.1$ $\mathrm{Hz}), 148.7(\mathrm{~d}, J=7.3 \mathrm{~Hz}), 135.9,133.6,132.8,132.0,131.2,130.3(\mathrm{~d}, J=3.4 \mathrm{~Hz}), 130.2(\mathrm{~d}, J=$ $3.4 \mathrm{~Hz}), 128.9(\mathrm{~d}, J=3.1 \mathrm{~Hz}), 128.4,127.4,126.3(\mathrm{~d}, J=4.0 \mathrm{~Hz}), 126.2(\mathrm{~d}, J=3.7 \mathrm{~Hz}), 123.6(\mathrm{~d}$, $J=3.3 \mathrm{~Hz}), 123.5(\mathrm{~d}, J=3.4 \mathrm{~Hz}), 115.0(\mathrm{~d}, J=4.4 \mathrm{~Hz}), 114.8(\mathrm{~d}, J=4.3 \mathrm{~Hz}), 52.8,52.5,45.1$, 44.9, 32.3, 31.9, 28.6, 28.2, 19.8, 19.8.

${ }^{19} \mathrm{~F} \mathrm{NMR}\left(376 \mathrm{MHz}, \mathrm{CDCl}_{3}\right) \delta-118.7$.

HRMS (APCI) Calculated for $\mathrm{C}_{19} \mathrm{H}_{20} \mathrm{~F}[\mathrm{M}+\mathrm{H}]^{+}: 267.1544$, found: 267.1541 .

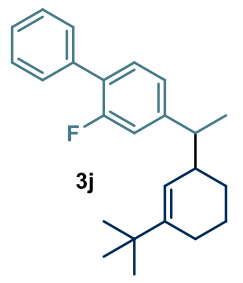

\section{4-(1-(3-(tert-butyl)cyclohex-2-en-1-yl)ethyl)-2-fluoro-1,1'-biphenyl (3j)}

Prepared according to General Procedure A using the radical precursor 1g (52.7 $\mathrm{mg}, 0.1 \mathrm{mmol})$ and 1-tert-Butyl-1-cyclohexene $2 \mathbf{b}(170 \mu \mathrm{L}, 1.0 \mathrm{mmol})$. The crude mixture was purified by column chromatography $\left(\mathrm{SiO}_{2}\right.$, hexanes) to afford product $\mathbf{3 j}$ as a colorless oil $(16 \mathrm{mg}, 48 \%$ yield, 1.1:1 dr, single regioisomer, average of two runs).

$\underline{{ }^{1} \mathrm{H} \mathrm{NMR}}\left(400 \mathrm{MHz}, \mathrm{CDCl}_{3}\right)$ 1.1:1 mixture of diastereoisomers $\delta 7.62-7.55(\mathrm{~m}, 2 \mathrm{H}), 7.46(\mathrm{ddt}$, $J=8.2,6.1,0.9 \mathrm{~Hz}, 2 \mathrm{H}), 7.41-7.35(\mathrm{~m}, 2 \mathrm{H}), 7.08-6.98(\mathrm{~m}, 2 \mathrm{H}), 5.60(\mathrm{~d}, J=2.6 \mathrm{~Hz}, 0.5 \mathrm{H})$, $5.29-5.26(\mathrm{~m}, 0.5 \mathrm{H}), 2.77-2.61(\mathrm{~m}, 1 \mathrm{H}), 2.32(\mathrm{dtq}, J=10.8,5.5,2.5 \mathrm{~Hz}, 1 \mathrm{H}), 2.05(\mathrm{dd}, J=$ $17.1,4.7 \mathrm{~Hz}, 1 \mathrm{H}), 1.99-1.85(\mathrm{~m}, 1 \mathrm{H}), 1.84-1.70(\mathrm{~m}, 1 \mathrm{H}), 1.54-1.39(\mathrm{~m}, 2 \mathrm{H}), 1.35$ (d, $J=7.0$ $\mathrm{Hz}, 1.5 \mathrm{H}), 1.30-1.28(\mathrm{~m}, 1.5 \mathrm{H}), 1.07(\mathrm{~s}, 4.5 \mathrm{H}), 1.00(\mathrm{~s}, 4.5 \mathrm{H}), 0.92(\mathrm{~d}, J=3.6 \mathrm{~Hz}, 1 \mathrm{H})$.

${ }^{13} \mathrm{C} \mathrm{NMR}\left(101 \mathrm{MHz}, \mathrm{CDCl}_{3}\right)$ 1.1:1 mixture of diastereoisomers $\delta 160.83,158.37,148.68(\mathrm{~d}, J=$ $7.2 \mathrm{~Hz}), 148.47(\mathrm{~d}, J=7.1 \mathrm{~Hz}), 136.01,130.06(\mathrm{dd}, J=7.9,4.0 \mathrm{~Hz}), 128.96(\mathrm{~d}, J=3.0 \mathrm{~Hz})$, $128.38,127.35,123.98(\mathrm{~d}, J=3.0 \mathrm{~Hz}), 123.68(\mathrm{~d}, J=3.2 \mathrm{~Hz}), 120.81,119.56,115.36,115.15$ (d, $J=3.2 \mathrm{~Hz}), 114.94,44.87,44.66,42.27,41.98,35.53,35.39,29.15,29.06,27.37,26.06,24.75$, 24.68, 22.77, 22.32, 18.53, 17.61 .

${ }^{19} \mathrm{~F}$ NMR $\left(376 \mathrm{MHz}, \mathrm{CDCl}_{3}\right) \delta-119.0$.

HRMS (APCI) Calculated for $\mathrm{C}_{20} \mathrm{H}_{30} \mathrm{~F}[\mathrm{M}+\mathrm{H}]^{+}: 337.2326$, found: 337.2324 .
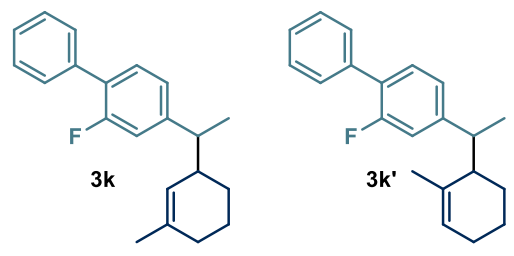

\section{2-fluoro-4-(1-(3-methylcyclohex-2-en-1-yl)ethyl)-1,1'- biphenyl (3k)}

Prepared according to General Procedure A using the radical precursor $1 \mathrm{~g}(52.7 \mathrm{mg}, \quad 0.1 \mathrm{mmol})$ and 1-Methyl-1cyclohexene $2 \mathbf{d}(237 \mu \mathrm{L}, 2.0 \mathrm{mmol})$. The crude mixture was purified by column chromatography $\left(\mathrm{SiO}_{2}\right.$, hexanes) to afford product $3 \mathbf{k}$ as a colorless oil (12.8 mg, 44\% yield, 1.5:1 r.r.; major regioisomer (3k) 1.4:1 d.r., minor regioisomer (3k') 1.5:1 d.r., average of two runs). Product 3k was isolated together with an unidentified isomeric product.

$\underline{{ }^{1} \mathrm{H} \mathrm{NMR}}\left(400 \mathrm{MHz}, \mathrm{CDCl}_{3}\right)$ major regioisomer $(3 \mathrm{k})$ 1.4:1 mixture of diastereoiomers $\delta 7.56$ (ddddd, $J=7.2,5.8,4.3,2.7,1.6 \mathrm{~Hz}), 7.48-7.39(\mathrm{~m}), 7.39-7.30(\mathrm{~m}), 7.06-6.94(\mathrm{~m}), 5.54-$ $5.48(\mathrm{~m}, 1.4 \mathrm{H}), 5.15(\mathrm{dd}, J=2.8,1.4 \mathrm{~Hz}, 1 \mathrm{H}), 2.59(\mathrm{p}, J=7.2 \mathrm{~Hz}, 2.4 \mathrm{H}), 2.40-2.18(\mathrm{~m}, 2.4 \mathrm{H})$, $1.99-1.84(\mathrm{~m}), 1.84-1.75(\mathrm{~m}), 1.69(\mathrm{dt}, J=2.3,1.1 \mathrm{~Hz}, 3 \mathrm{H}), 1.62(\mathrm{dt}, J=2.4,1.2 \mathrm{~Hz}, 3 \mathrm{H})$, $1.72-1.60(\mathrm{~m}), 1.47$ (dddd, $J=15.0,10.4,5.5,2.7 \mathrm{~Hz}), 1.32(\mathrm{~d}, J=6.9 \mathrm{~Hz}, 3 \mathrm{H}), 1.25(\mathrm{~d}, J=7.0$ $\mathrm{Hz}, 3 \mathrm{H}), 1.37-1.24$ (m).

$\underline{{ }^{1} \mathrm{H} \mathrm{NMR}}\left(400 \mathrm{MHz}, \mathrm{CDCl}_{3}\right)$ minor regioisomer (3k') 1.5:1 mixture of diastereoiomers $\delta 7.56$ (ddddd, $J=7.2,5.8,4.3,2.7,1.6 \mathrm{~Hz}), 7.48-7.39(\mathrm{~m}), 7.39-7.30(\mathrm{~m}), 7.06-6.94(\mathrm{~m}), 5.66(\mathrm{~s}$, $1.5 \mathrm{H}), 5.58(\mathrm{~s}, 1 \mathrm{H}), 2.79(\mathrm{q}, J=7.2 \mathrm{~Hz}, 1 \mathrm{H}), 2.69(\mathrm{qd}, J=7.4,3.8 \mathrm{~Hz}, 1.5 \mathrm{H}), 1.99-1.84(\mathrm{~m})$, $1.84-1.75(\mathrm{~m}), 1.71(\mathrm{~s}, 3 \mathrm{H}), 1.72-1.60(\mathrm{~m}), 1.54(\mathrm{~s}, 4.5 \mathrm{H}), 1.47$ (dddd, $J=15.0,10.4,5.5,2.7$ $\mathrm{Hz}), 1.31(\mathrm{~d}, J=7.1 \mathrm{~Hz}, 3 \mathrm{H}), 1.28(\mathrm{~d}, J=7.3 \mathrm{~Hz}, 4.5 \mathrm{H}) 1.37-1.24(\mathrm{~m})$. 
${ }^{13} \mathrm{C} \mathrm{NMR}\left(126 \mathrm{MHz}, \mathrm{CDCl}_{3}\right)$ mixture of regioisomers and diasteroisomers $\delta 160.6,158.7,136.0$, 135.8, 135.0, 130.2, 130.2, 128.9, 128.9, 128.4, 128.4, 127.4, 127.3, 124.4, 123.8, 123.8, 123.6, 123.6, 123.2, 115.3, 115.1, 115.0, 114.8, 49.3, 47.8, 45.3, 45.0, 44.8, 44.5, 42.0, 42.0, 33.6, 31.8, $30.2,30.1,27.4,26.2,25.4,25.1,25.1,24.5,24.1,23.9,22.3,21.7,19.1,18.7,18.5,15.9,15.6$, 14.1, 12.8 .

${ }^{19} \mathrm{~F} \mathrm{NMR}\left(376 \mathrm{MHz}, \mathrm{CDCl}_{3}\right) \delta-118.9,-119.0,-119.1,-119.4$.

HRMS (APCI) Calculated for $\mathrm{C}_{21} \mathrm{H}_{23} \mathrm{~F}[\mathrm{M}+\mathrm{H}]^{+}: 295.1857$, found: 295.1858 .<smiles>CC(=O)C(c1ccccc1)C1C=CCCC1</smiles>

\section{methyl 2-(cyclohex-2-en-1-yl)-2-phenylacetate (31)}

Prepared according to General Procedure A using the radical precursor 4a (109 $\mathrm{mg}, 0.2 \mathrm{mmol})$, and cyclohexene $2 \mathrm{a}(203 \mu \mathrm{L}, 2.0 \mathrm{mmol})$. The crude mixture was purified by column chromatography $\left(\mathrm{SiO}_{2}, 50: 50\right.$ hexanes/toluene) to afford product $\mathbf{3 1}$ as a colorless oil (14.6 mg, 32\% yield, 1.1:1 dr, average of two runs).

${ }^{1} \mathrm{H}$ NMR $\left(500 \mathrm{MHz}, \mathrm{CDCl}_{3}\right) 1.1: 1$ mixture of diastereoiomers $\delta 7.37-7.30(\mathrm{~m}, 4 \mathrm{H}), 7.29-7.24$ $(\mathrm{m}, 1 \mathrm{H}), 5.79(\mathrm{dtd}, J=9.8,3.7,2.0 \mathrm{~Hz}, 0.5 \mathrm{H}), 5.67-5.60(\mathrm{~m}, 1 \mathrm{H}), 5.18-5.13(\mathrm{~m}, 0.5 \mathrm{H}), 3.67$ (s, 3H), $3.66(\mathrm{~s}, 3 \mathrm{H}), 3.32(\mathrm{dd}, J=11.2,1.1 \mathrm{~Hz}, 1 \mathrm{H}), 2.86(\mathrm{dtdt}, J=18.8,8.0,5.2,2.6 \mathrm{~Hz}, 1 \mathrm{H})$, $1.98(\mathrm{tt}, J=5.7,4.0,2.0 \mathrm{~Hz}, 2 \mathrm{H}), 1.93-1.86(\mathrm{~m}, 1 \mathrm{H}), 1.82-1.71(\mathrm{~m}, 1 \mathrm{H}), 1.67-1.54(\mathrm{~m}, 1 \mathrm{H})$, $1.46(\mathrm{dtt}, J=13.3,6.5,3.0 \mathrm{~Hz}, 1 \mathrm{H}), 1.42-1.27$ (m, 1H), 1.06 (dddd, $J=13.3,10.7,8.0,2.9 \mathrm{~Hz}$, $1 \mathrm{H})$.

${ }^{13} \mathrm{C} \mathrm{NMR}\left(126 \mathrm{MHz}, \mathrm{CDCl}_{3}\right) 1.1: 1$ mixture of diastereoiomers $\delta 174.0,174.0,137.6,137.4$, $129.2,128.9,128.8,128.7,128.6,128.5,128.4,127.9,127.3,57.5,57.4,51.8,51.8,38.5,38.4$, 29.7, 27.9, 26.3, 25.3, 25.2, 21.1, 20.7.

Analytical data are in agreement with the one reported in literature. ${ }^{23}$

\section{D.3 Three-component C-H Allylic Benzylation \\ D.3.1 Optimization Data}

To an argon-purged glass vial, containing the thiol catalyst, the radical precursor $\mathbf{4 b}$ ( $48.1 \mathrm{mg}, 0.1$ mmol), and $\mathrm{Na}_{2} \mathrm{HPO}_{4}(2.8 \mathrm{mg}, 0.02 \mathrm{mmol}, 0.2$ equiv.), was added the radical trap $\mathbf{5 a}(17.7 \mu \mathrm{L}$, 0.1 mmol, 1 equiv.) and cyclohexene 2a. Then, $800 \mu \mathrm{L}$ of argon-sparged HPLC grade acetone were added. The vial was sealed with Parafilm, and then placed in the irradiation setup, maintained at the defined temperature. The reaction was stirred for the stated amount of time, then the solvent was evaporated and the crude mixture purified by flash column chromatography on silica gel to furnish the product $6 \mathbf{a}$.

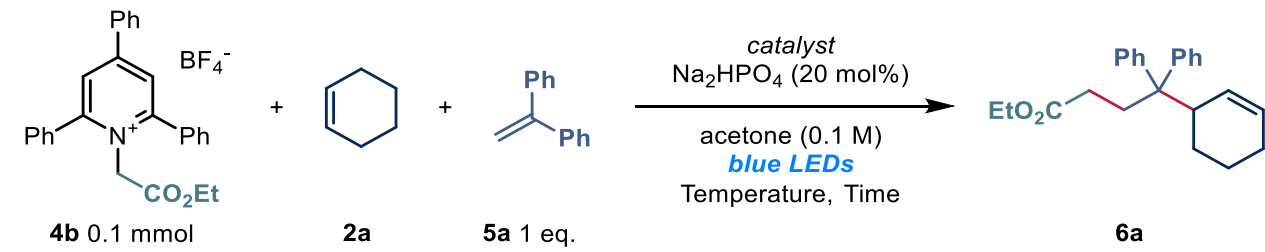

\begin{tabular}{|c|c|c|c|c|c|c|}
\hline entry & loading $\mathbf{2 a}$ & catalyst (loading) & $\mathrm{T}\left({ }^{\circ} \mathrm{C}\right)$ & time $(h)$ & conversion $(\%)^{\mathrm{a}}$ & yield $\mathbf{6 a}(\%)^{a}$ \\
\hline 1 & 20 equiv. & C5 (20 mol\%) & 35 & 18 & 75 & 56 \\
\hline 2 & 20 equiv. & C5 (20 mol\%) & 35 & 48 & 100 & $75(65)^{c}$ \\
\hline 3 & 10 equiv. & C5 (20 mol\%) & 35 & 24 & 100 & 85 \\
\hline
\end{tabular}




$\begin{array}{llllllc}4 & 20 \text { equiv. } & \text { C5 (10 mol\%) } & 35 & 24 & 100 & 88 \\ 5 & 10 \text { equiv. } & \text { C5 (10 mol\%) } & 35 & 24 & 80 & 67 \\ 6 & 10 \text { equiv. } & \text { C5 (10 mol\%) } & 35 & 28 & 100 & 85(78)^{b} \\ 7 & 10 \text { equiv. } & \text { C5 (10 mol\%) } & 60 & 8 & 100 & 96(95)^{b} \\ 8 & 5 \text { equiv. } & \text { C5 (10 mol\%) } & 60 & 14 & 90 & 70 \\ 9 & 10 \text { equiv. } & \text { C6 (10 mol\%) } & 60 & 8 & 100 & 88 \\ 10 & 10 \text { equiv. } & \text { C7 (10 mol\%) } & 60 & 14 & 35 & 30 \\ 11 & 10 \text { equiv. } & \text { C4 (10 mol\%) } & 35 & 28 & 100 & 40 \\ 12^{a} & 10 \text { equiv. } & \text { C5 (10 mol\%) } & 60 & 28 & - & - \\ 13 & 10 \text { equiv. } & - & 60 & 28 & - & -\end{array}$

Table S1. Optimization studies for the three-component $\mathrm{C}-\mathrm{H}$ allylic benzylation. ${ }^{\text {a }}$ Yield determined by ${ }^{1} \mathrm{H}$ NMR analysis. ${ }^{\text {b }}$ Yield of the isolated $\mathbf{6 a}$.

\section{D.3.2 General Procedure B}

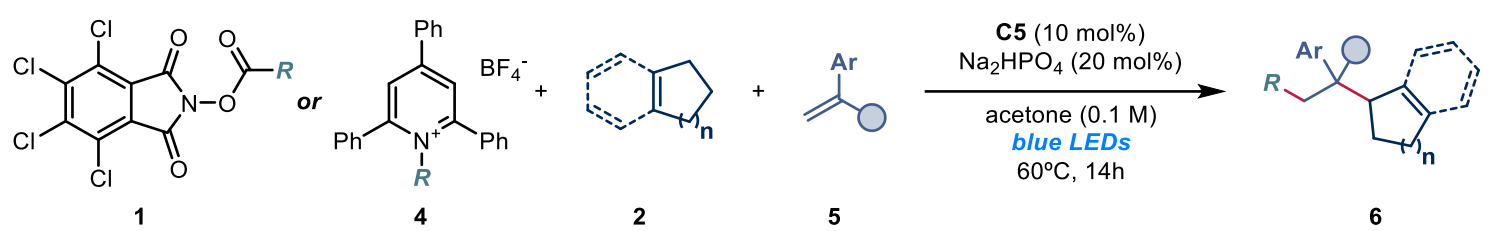

To an argon-purged glass vial, containing the catalyst $\mathbf{C 5}$ (3.8 $\mathrm{mg}, 0.01 \mathrm{mmol}, 0.1$ equiv.), tetrachloro-phthalimide ester 1 or pyridinium salt 4 ( $0.1 \mathrm{mmol}, 1$ equiv.), and $\mathrm{Na}_{2} \mathrm{HPO}_{4}(2.8 \mathrm{mg}$, $0.02 \mathrm{mmol}, 0.2$ equiv.), was added the alkene 5 ( $0.1 \mathrm{mmol}, 1$ equiv.) and the allylic precursor $\mathbf{2}$ ( $1 \mathrm{mmol}, 10$ equiv.). Then, $800 \mu \mathrm{L}$ of argon-sparged HPLC grade acetone were added. The vial was sealed with Parafilm, and then placed in the irradiation setup, maintained at a temperature of $60{ }^{\circ} \mathrm{C}$. The reaction was stirred for $14 \mathrm{~h}$, then the solvent was evaporated and the crude mixture purified by flash column chromatography on silica gel to furnish the product 6 .

\section{D.3.3 Characterization of Products 6}

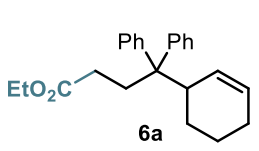

\section{Ethyl 4-(cyclohex-2-en-1-yl)-4,4-diphenylbutanoate (6a)}

Prepared according to General Procedure B using the radical precursor $\mathbf{4 b}$ (48.1 $\mathrm{mg}, 0.1 \mathrm{mmol})$, cyclohexene 2a $(101 \mu \mathrm{L}, 1.0 \mathrm{mmol})$, and 1,1diphenylethylene 5a $(17.7 \mu \mathrm{L}, 0.1 \mathrm{mmol})$. The crude mixture was purified by column chromatography $\left(\mathrm{SiO}_{2}, 50: 50\right.$ hexanes/toluene) to afford product $6 \mathbf{a}$ as a colorless oil (33.0 $\mathrm{mg}$, $95 \%$ yield, average of two runs).

${ }^{1} \mathrm{H}$ NMR $\left(400 \mathrm{MHz}, \mathrm{CDCl}_{3}\right) \delta 7.33-7.20(\mathrm{~m}, 10 \mathrm{H}), 5.81(\mathrm{dt}, \mathrm{J}=10.4,2.1 \mathrm{~Hz}, 1 \mathrm{H}), 5.62$ (dddd, $\mathrm{J}=9.8,5.1,2.7,1.3 \mathrm{~Hz}, 1 \mathrm{H}), 4.07(\mathrm{q}, \mathrm{J}=7.2 \mathrm{~Hz}, 2 \mathrm{H}), 3.19$ (ddt, $\mathrm{J}=9.3,6.9,2.4 \mathrm{~Hz}, 1 \mathrm{H}), 2.59$ $(\mathrm{ddd}, \mathrm{J}=13.9,11.8,4.6 \mathrm{~Hz}, 1 \mathrm{H}), 2.48-2.37(\mathrm{~m}, 1 \mathrm{H}), 2.07(\mathrm{ddd}, \mathrm{J}=16.3,11.7,4.6 \mathrm{~Hz}, 1 \mathrm{H}), 1.98$ $-1.82(\mathrm{~m}, 3 \mathrm{H}), 1.76-1.49$ (m, 3H), 1.22 (t, J = 7.1 Hz, 3H), 0.94 (tdd, $J=12.5,10.4,3.6 \mathrm{~Hz}$, $1 \mathrm{H})$.

${ }^{13} \mathrm{C} \mathrm{NMR}\left(126 \mathrm{MHz}, \mathrm{CDCl}_{3}\right) \delta 173.9,145.2,142.9,129.7,129.3,129.0,128.9,127.7,127.1$, 126.0, 125.9, 60.3, 53.7, 41.6, 33.9, 30.1, 25.0, 24.7, 22.4, 14.2.

$\underline{\mathrm{HRMS}}$ (APCI) Calculated for $\mathrm{C}_{24} \mathrm{H}_{29} \mathrm{O}_{2}[\mathrm{M}+\mathrm{H}]^{+}: 349.2162$, found: 349.2164 . 


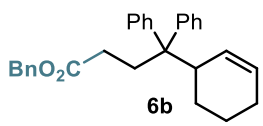

\section{Benzyl 4-(cyclohex-2-en-1-yl)-4,4-diphenylbutanoate (6b)}

Prepared according to General Procedure B using the radical precursor $\mathbf{4 c}$ (54.3 $\mathrm{mg}, 0.1 \mathrm{mmol})$, cyclohexene $2 \mathbf{a}(101 \mu \mathrm{L}, 1.0 \mathrm{mmol})$, and 1,1diphenylethylene 5a $(17.7 \mu \mathrm{L}, 0.1 \mathrm{mmol})$. The crude mixture was purified by column chromatography ( $\mathrm{SiO}_{2}, 70: 30$ hexanes/toluene) to afford product $\mathbf{6 b}$ as a colorless oil (27.7 $\mathrm{mg}$, $68 \%$ yield, average of two runs). Multiple purifications by column chromatography resulted in poor separation from several unidentified byproducts. The yield (75\%, average of two runs) of $\mathbf{6 b}$ was inferred by ${ }^{1} \mathrm{H}$ NMR analysis of the crude reaction mixture using trichloroethylene as the internal standard. An analytical amount of pure compound was isolated by preparative TLC (80:20 hexanes/toluene) to obtain a colorless oil.

$\underline{{ }^{1} \mathrm{H} \mathrm{NMR}}\left(400 \mathrm{MHz}, \mathrm{CDCl}_{3}\right) \delta 7.42-7.19(\mathrm{~m}, 15 \mathrm{H}), 5.82(\mathrm{dq}, \mathrm{J}=10.4,2.0 \mathrm{~Hz}, 1 \mathrm{H}), 5.62(\mathrm{ddt}, \mathrm{J}$ $=10.2,4.9,2.4 \mathrm{~Hz}, 1 \mathrm{H}), 5.06(\mathrm{~s}, 2 \mathrm{H}), 3.25-3.16(\mathrm{~m}, 1 \mathrm{H}), 2.63(\mathrm{ddd}, \mathrm{J}=13.9,11.7,4.6 \mathrm{~Hz}, 1 \mathrm{H})$, $2.46(\mathrm{ddd}, \mathrm{J}=13.9,11.6,5.3 \mathrm{~Hz}, 1 \mathrm{H}), 2.22-2.10(\mathrm{~m}, 1 \mathrm{H}), 2.02(\mathrm{ddd}, \mathrm{J}=16.5,11.7,5.3 \mathrm{~Hz}, 1 \mathrm{H})$, $1.90(\mathrm{tt}, \mathrm{J}=15.0,2.9 \mathrm{~Hz}, 2 \mathrm{H}), 1.77-1.49(\mathrm{~m}, 3 \mathrm{H}), 0.98(\mathrm{tdd}, \mathrm{J}=12.3,10.3,3.7 \mathrm{~Hz}, 1 \mathrm{H})$.

${ }^{13} \mathrm{C} \mathrm{NMR}\left(101 \mathrm{MHz}, \mathrm{CDCl}_{3}\right) \delta 173.7,145.2,142.8,135.9,129.7,129.3,129.0,128.9,128.5$, 128.2, 128.2, 127.8, 127.7, 127.1, 127.1, 126.0, 125.9, 66.2, 53.8, 41.6, 33.9, 30.1, 25.0, 24.7, 22.4. HRMS (ESI) Calculated for $\mathrm{C}_{29} \mathrm{H}_{30} \mathrm{NaO}_{2}[\mathrm{M}+\mathrm{Na}]^{+}$: 433.2138, found: 433.2129 .

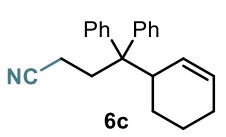

\section{4-(cyclohex-2-en-1-yl)-4,4-diphenylbutanenitrile (6c)}

Prepared according to General Procedure B using the radical precursor 4d (43.4 $\mathrm{mg}, 0.1 \mathrm{mmol})$, cyclohexene $2 \mathrm{a}(101 \mu \mathrm{L}, 1.0 \mathrm{mmol})$, and 1,1-diphenylethylene 5a $(17.7 \mu \mathrm{L}, 0.1 \mathrm{mmol})$. The crude mixture was purified by column chromatography $\left(\mathrm{SiO}_{2}, 50: 50\right.$ hexanes/toluene) to afford product $\mathbf{6 c}$ as a colorless oil ( $28.1 \mathrm{mg}, 93 \%$ yield, average of two runs). ${ }^{1} \mathrm{H} \mathrm{NMR}\left(400 \mathrm{MHz}, \mathrm{CDCl}_{3}\right) \delta 7.37-7.24(\mathrm{~m}, 6 \mathrm{H}), 7.21(\mathrm{ddd}, \mathrm{J}=7.1,3.2,1.8 \mathrm{~Hz}, 4 \mathrm{H}), 5.76(\mathrm{dt}$, $\mathrm{J}=10.5,2.0 \mathrm{~Hz}, 1 \mathrm{H}), 5.69-5.61(\mathrm{~m}, 1 \mathrm{H}), 3.23-3.12(\mathrm{~m}, 1 \mathrm{H}), 2.65(\mathrm{ddd}, \mathrm{J}=14.0,11.8,4.6 \mathrm{~Hz}$, $1 \mathrm{H}), 2.47(\mathrm{ddd}, \mathrm{J}=13.9,11.7,5.5 \mathrm{~Hz}, 1 \mathrm{H}), 2.08(\mathrm{ddd}, \mathrm{J}=16.4,11.7,4.5 \mathrm{~Hz}, 1 \mathrm{H}), 1.96-1.82(\mathrm{~m}$, $3 \mathrm{H}), 1.75-1.52$ (m, 3H), 0.98 (tdd, J = 12.1, 10.3, 3.7 Hz, 1H).

${ }^{13} \mathrm{C} \mathrm{NMR}\left(101 \mathrm{MHz}, \mathrm{CDCl}_{3}\right) \delta 143.97,141.62,129.58,129.42,129.09,128.23,128.14,127.51$, 126.56, 126.52, 120.07, 53.97, 41.54, 35.09, 24.93, 24.73, 22.23, 13.25, 1.03.

HRMS (APCI) Calculated for $\mathrm{C}_{22} \mathrm{H}_{24} \mathrm{~N}[\mathrm{M}+\mathrm{H}]^{+}: 302.1903$, found: 302.1902 .

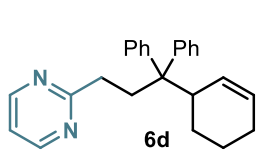

\section{2-(3-(cyclohex-2-en-1-yl)-3,3-diphenylpropyl)pyrimidine (6d)}

Prepared according to General Procedure B using the radical precursor $4 \mathbf{e}$ (48.7 $\mathrm{mg}, 0.1 \mathrm{mmol})$, cyclohexene $2 \mathrm{a}(101 \mu \mathrm{L}, 1.0 \mathrm{mmol})$, and 1,1diphenylethylene 5a $(17.7 \mu \mathrm{L}, 0.1 \mathrm{mmol})$. The crude mixture was purified by column chromatography $\left(\mathrm{SiO}_{2}, 80: 20\right.$ hexanes/EtOAc) to afford product $\mathbf{6 d}$ as a white solid (14.5 $\mathrm{mg}$, $41 \%$ yield, average of two runs).

${ }^{1} \mathrm{H} \mathrm{NMR}\left(400 \mathrm{MHz}, \mathrm{CDCl}_{3}\right) \delta 8.63(\mathrm{~d}, J=4.9 \mathrm{~Hz}, 2 \mathrm{H}), 7.37-7.27(\mathrm{~m}, 8 \mathrm{H}), 7.26-7.18(\mathrm{~m}, 2 \mathrm{H})$, $7.09(\mathrm{t}, J=4.9 \mathrm{~Hz}, 1 \mathrm{H}), 5.88(\mathrm{dt}, J=10.4,2.1 \mathrm{~Hz}, 1 \mathrm{H}), 5.66-5.58(\mathrm{~m}, 1 \mathrm{H}), 3.32(\mathrm{ddd}, J=11.0$, $5.5,3.0 \mathrm{~Hz}, 1 \mathrm{H}), 2.80-2.67(\mathrm{~m}, 2 \mathrm{H}), 2.66-2.57(\mathrm{~m}, 2 \mathrm{H}), 1.98(\mathrm{dd}, J=12.5,3.7 \mathrm{~Hz}, 1 \mathrm{H}), 1.84$ (s, 1H), $1.74-1.64(\mathrm{~m}, 1 \mathrm{H}), 1.56(\mathrm{ddt}, J=13.7,10.7,5.5 \mathrm{~Hz}, 2 \mathrm{H}), 1.04-0.96(\mathrm{~m}, 1 \mathrm{H})$.

${ }^{13} \mathrm{C}$ NMR $\left(101 \mathrm{MHz}, \mathrm{CDCl}_{3}\right) \delta 171.7,156.9,145.7,143.4,129.9,129.4,129.4,128.7,127.7$, 127.0, 125.8, 125.8, 118.3, 54.2, 41.6, 37.8, 35.0, 25.0, 24.7, 22.4.

$\underline{\text { HRMS }}$ (ESI) Calculated for $\mathrm{C}_{25} \mathrm{H}_{26} \mathrm{NaN}_{2}[\mathrm{M}+\mathrm{Na}]^{+}: 355.2169$, found: 355.2175 . 


\section{(1-(cyclohex-2-en-1-yl)-2-cyclohexylethane-1,1-diyl)dibenzene (6e)}

Prepared according to General Procedure B using the radical precursor $\mathbf{1 h}$ (49.3 $\mathrm{mg}, 0.12 \mathrm{mmol})$, cyclohexene $2 \mathrm{a}(101 \mu \mathrm{L}, 1.0 \mathrm{mmol})$, and 1,1-diphenylethylene

5a $(17.7 \mu \mathrm{L}, 0.1 \mathrm{mmol})$. The crude mixture was purified by column chromatography $\left(\mathrm{SiO}_{2}\right.$, pentane) to afford product $6 \mathbf{e}$ as a colorless oil ( $24.2 \mathrm{mg}, 70 \%$ yield, average of two runs).

${ }^{1} \mathrm{H} \mathrm{NMR}\left(400 \mathrm{MHz}, \mathrm{CDCl}_{3}\right) \delta 7.30-7.19(\mathrm{~m}, 10 \mathrm{H}), 5.94-5.88(\mathrm{~m}, 1 \mathrm{H}), 5.60$ (ddt, $J=10.1,5.0$, $2.5 \mathrm{~Hz}, 1 \mathrm{H}), 3.22$ (ddq, $J=9.7,4.7,2.3 \mathrm{~Hz}, 1 \mathrm{H}), 2.08(\mathrm{dd}, J=14.1,3.9 \mathrm{~Hz}, 1 \mathrm{H}), 2.01-1.96(\mathrm{~m}$, $1 \mathrm{H}), 1.93-1.83(\mathrm{~m}, 1 \mathrm{H}), 1.74-1.61(\mathrm{~m}, 2 \mathrm{H}), 1.48(\mathrm{ddd}, J=12.3,8.2,5.1 \mathrm{~Hz}, 3 \mathrm{H}), 1.17(\mathrm{dtd}, J$ $=14.8,6.8,3.8 \mathrm{~Hz}, 2 \mathrm{H}), 1.11-0.98(\mathrm{~m}, 4 \mathrm{H}), 0.91(\mathrm{tdd}, J=10.1,5.6,2.2 \mathrm{~Hz}, 3 \mathrm{H}), 0.85-0.69$ $(\mathrm{m}, 2 \mathrm{H})$.

${ }^{13} \mathrm{C} \mathrm{NMR}\left(101 \mathrm{MHz}, \mathrm{CDCl}_{3}\right) \delta 146.2,144.7,129.9,129.8,129.6,128.6,127.3,126.8,125.7$, $125.6,54.9,46.7,41.4,3.5,35.2,33.7,33.3,26.6,26.5,26.3,25.1,22.5$.

HRMS (APCI) Calculated for $\mathrm{C}_{26} \mathrm{H}_{33}[\mathrm{M}+\mathrm{H}]^{+}: 345.2577$, found: 345.2578 .

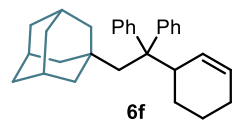

\section{1-(2-(cyclohex-2-en-1-yl)-2,2-diphenylethyl)adamantane (6f)}

Prepared according to General Procedure B using the radical precursor 1i (55.6 $\mathrm{mg}, 0.12 \mathrm{mmol})$, cyclohexene $2 \mathrm{a}(101 \mu \mathrm{L}, 1.0 \mathrm{mmol})$, and 1,1-diphenylethylene

5a $(17.7 \mu \mathrm{L}, 0.1 \mathrm{mmol})$. The crude mixture was purified by column chromatography $\left(\mathrm{SiO}_{2}\right.$, pentane) to afford product $\mathbf{6} \mathbf{f}$ as a colorless oil ( $23.0 \mathrm{mg}, 58 \%$ yield, average of two runs).

${ }^{1} \mathrm{H} \mathrm{NMR}\left(500 \mathrm{MHz}, \mathrm{CDCl}_{3}\right) \delta 7.38-7.14(\mathrm{~m}, 10 \mathrm{H}), 5.89(\mathrm{dt}, J=10.6,2.1 \mathrm{~Hz}, 1 \mathrm{H}), 5.51$ (ddt, $J$ $=10.3,5.0,2.5 \mathrm{~Hz}, 1 \mathrm{H}), 3.46(\mathrm{ddh}, J=9.5,4.8,2.5 \mathrm{~Hz}, 1 \mathrm{H}), 2.18(\mathrm{~d}, J=14.8 \mathrm{~Hz}, 1 \mathrm{H}), 2.07(\mathrm{~d}$, $J=15.2 \mathrm{~Hz}, 1 \mathrm{H}), 2.05-2.01(\mathrm{~m}, 2 \mathrm{H}), 1.88-1.78(\mathrm{~m}, 1 \mathrm{H}), 1.71(\mathrm{p}, J=3.2 \mathrm{~Hz}, 3 \mathrm{H}), 1.67-1.49$ $(\mathrm{m}, 7 \mathrm{H}), 1.43(\mathrm{dq}, J=12.2,2.1 \mathrm{~Hz}, 3 \mathrm{H}), 1.30-1.23(\mathrm{~m}, 2 \mathrm{H}), 1.16(\mathrm{dq}, J=12.3,2.7 \mathrm{~Hz}, 3 \mathrm{H})$.

${ }^{13} \mathrm{C} \mathrm{NMR}\left(126 \mathrm{MHz}, \mathrm{CDCl}_{3}\right) \delta 146.2,145.6,130.4,130.2,129.7,128.0,127.9,127.6,127.1$, $126.8,126.3,125.7,125.5,54.0,52.4,43.5,43.1,41.2$, 36.8, 36.7, 34.7, 29.7, 28.9, 28.5, 25.4, 25.0, 22.4 .

$\underline{\text { HRMS }}$ (APCI) Calculated for $\mathrm{C}_{30} \mathrm{H}_{36}[\mathrm{M}+\mathrm{H}]^{+}:$397.2890, found: 397.2887.

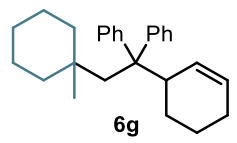

\section{(1-(cyclohex-2-en-1-yl)-2-(1-methylcyclohexyl)ethane-1,1-diyl)dibenzene $(6 \mathrm{~g})$}

Prepared according to General Procedure B using the radical precursor $1 \mathbf{j}$ (51.0 $\mathrm{mg}, 0.12 \mathrm{mmol})$, cyclohexene $\mathbf{2 a}(101 \mu \mathrm{L}, 1.0 \mathrm{mmol})$, and 1,1-diphenylethylene 5a $(17.7 \mu \mathrm{L}, 0.1$ mmol). The crude mixture was purified by column chromatography $\left(\mathrm{SiO}_{2}\right.$, pentane) to afford product $\mathbf{6 g}$ as a colorless oil ( $21.9 \mathrm{mg}, 58 \%$ yield, average of two runs).

${ }^{1} \mathrm{H} \mathrm{NMR}\left(400 \mathrm{MHz}, \mathrm{CDCl}_{3}\right) \delta 7.37-7.30(\mathrm{~m}, 2 \mathrm{H}), 7.31-7.14(\mathrm{~m}, 8 \mathrm{H}), 5.86(\mathrm{dt}, J=10.5,2.0$ $\mathrm{Hz}, 1 \mathrm{H}), 5.51(\mathrm{ddt}, J=10.3,5.1,2.5 \mathrm{~Hz}, 1 \mathrm{H}), 3.57-3.38(\mathrm{~m}, 1 \mathrm{H}), 2.31(\mathrm{~d}, J=14.8 \mathrm{~Hz}, 1 \mathrm{H}), 2.26$ $(\mathrm{d}, J=15.0 \mathrm{~Hz}, 1 \mathrm{H}), 2.10-1.97(\mathrm{~m}, 1 \mathrm{H}), 1.82(\mathrm{dt}, J=19.3,3.2 \mathrm{~Hz}, 1 \mathrm{H}), 1.67-1.47(\mathrm{~m}, 4 \mathrm{H})$, 1.33 (tdd, $J=9.5,6.8,3.8 \mathrm{~Hz}, 3 \mathrm{H}), 1.24-1.05(\mathrm{~m}, 4 \mathrm{H}), 0.96(\mathrm{dd}, J=7.2,3.8 \mathrm{~Hz}, 2 \mathrm{H}), 0.93-$ $0.86(\mathrm{~m}, 2 \mathrm{H}), 0.83(\mathrm{t}, J=5.9 \mathrm{~Hz}, 2 \mathrm{H}), 0.65(\mathrm{~s}, 3 \mathrm{H})$.

${ }^{13} \mathrm{C} \mathrm{NMR}\left(101 \mathrm{MHz}, \mathrm{CDCl}_{3}\right) \delta 145.8,145.5,130.2,129.6,128.0,127.0,126.2,125.6,125.5,54.2$, 41.5, 39.8, 39.8, 34.7, 29.6, 26.2, 25.3, 25.0, 22.4, 21.9, 21.9.

HRMS (APCI) Calculated for $\mathrm{C}_{27} \mathrm{H}_{35}[\mathrm{M}+\mathrm{H}]^{+}: 359.2733$, found: 359.2730 . 


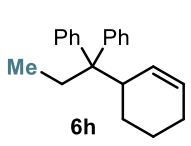

(1-(cyclohex-2-en-1-yl)propane-1,1-diyl)dibenzene (6h)

Prepared according to General Procedure B using the radical precursor 1k (41.2 $\mathrm{mg}, 0.12 \mathrm{mmol})$, cyclohexene $\mathbf{2 a}(101 \mu \mathrm{L}, 1.0 \mathrm{mmol})$, and 1,1-diphenylethylene

5a $(17.7 \mu \mathrm{L}, 0.1 \mathrm{mmol})$. The crude mixture was purified by column chromatography $\left(\mathrm{SiO}_{2}\right.$, pentane) to afford product $\mathbf{6 h}$ as a colorless oil (17.8 $\mathrm{mg}, 64 \%$ yield, average of two runs).

${ }^{1} \mathrm{H}$ NMR $\left(400 \mathrm{MHz}, \mathrm{CDCl}_{3}\right) \delta 7.31-7.21(\mathrm{~m}, 10 \mathrm{H}), 5.90-5.84(\mathrm{~m}, 1 \mathrm{H}), 5.64-5.58(\mathrm{~m}, 1 \mathrm{H})$, 3.22 (ddq, $\mathrm{J}=9.8,4.8,2.4 \mathrm{~Hz}, 1 \mathrm{H}), 2.22-2.16(\mathrm{~m}, 2 \mathrm{H}), 1.88(\mathrm{dddd}, \mathrm{J}=14.0,5.8,2.7,1.4 \mathrm{~Hz}$, $2 \mathrm{H}), 1.74-1.62(\mathrm{~m}, 1 \mathrm{H}), 1.03-0.84(\mathrm{~m}, 3 \mathrm{H}), 0.64(\mathrm{t}, \mathrm{J}=7.3 \mathrm{~Hz}, 3 \mathrm{H})$.

${ }^{13} \mathrm{C}$ NMR $\left(101 \mathrm{MHz}, \mathrm{CDCl}_{3}\right) \delta 146.0,144.2,129.9,129.6,129.6,128.5,127.3,126.8,125.6$, $125.4,54.5,40.3,31.6,25.0,24.6,22.5,8.9$.

HRMS (APCI) Calculated for $\mathrm{C}_{21} \mathrm{H}_{25}[\mathrm{M}+\mathrm{H}]^{+}: 277.1951$, found: 277.1948 .

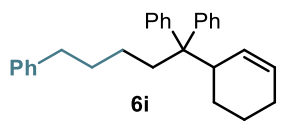

\section{(1-(cyclohex-2-en-1-yl)pentane-1,1,5-triyl)tribenzene (6i)}

Prepared according to General Procedure B using the radical precursor $\mathbf{1 l}$ $(53.7 \mathrm{mg}, 0.12 \mathrm{mmol})$, cyclohexene $2 \mathrm{a}(101 \mu \mathrm{L}, 1.0 \mathrm{mmol})$, and 1,1diphenylethylene 5a $(17.7 \mu \mathrm{L}, 0.1 \mathrm{mmol})$. The crude mixture was purified by column chromatography $\left(\mathrm{SiO}_{2}, 95: 5\right.$ hexanes $\left./ \mathrm{CH}_{2} \mathrm{Cl}_{2}\right)$ to afford product $\mathbf{6} \mathbf{i}$ as a colorless oil $(17.2 \mathrm{mg}$, $45 \%$ yield, average of two runs).

${ }^{1} \mathrm{H}$ NMR $\left(500 \mathrm{MHz}, \mathrm{CDCl}_{3}\right) \delta 7.31-7.20(\mathrm{~m}, 13 \mathrm{H}), 7.13-7.09(\mathrm{~m}, 2 \mathrm{H}), 5.85(\mathrm{dp}, \mathrm{J}=10.5,1.8$ $\mathrm{Hz}, 1 \mathrm{H}), 5.64-5.58(\mathrm{~m}, 1 \mathrm{H}), 3.21(\mathrm{ddh}, \mathrm{J}=9.3,4.6,2.4 \mathrm{~Hz}, 1 \mathrm{H}), 2.54-2.49(\mathrm{~m}, 2 \mathrm{H}), 2.19-$ $2.14(\mathrm{~m}, 2 \mathrm{H}), 1.92-1.83(\mathrm{~m}, 2 \mathrm{H}), 1.72-1.64(\mathrm{~m}, 1 \mathrm{H}), 1.61-1.51(\mathrm{~m}, 4 \mathrm{H}), 1.14-1.03(\mathrm{~m}, 2 \mathrm{H})$, 0.97 (tdd, $\mathrm{J}=12.1,10.3,3.8 \mathrm{~Hz}, 1 \mathrm{H})$.

${ }^{13} \mathrm{C} \mathrm{NMR}\left(101 \mathrm{MHz}, \mathrm{CDCl}_{3}\right) \delta 146.3,144.3,142.7,129.8,129.5,129.5,128.5,128.2,128.2$, $127.4,126.9,125.7,125.5,125.5,54.3,40.9,39.1,35.7,32.2,25.0,24.7,24.1,22.5$.

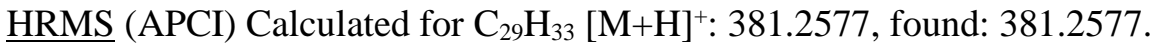

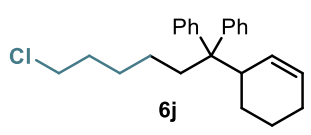

\section{(6-chloro-1-(cyclohex-2-en-1-yl)hexane-1,1-diyl)dibenzene (6j)}

Prepared according to General Procedure B using the radical precursor $1 \mathbf{m}$ (50.3 $\mathrm{mg}, 0.12 \mathrm{mmol})$, cyclohexene $2 \mathrm{a}(101 \mu \mathrm{L}, 1.0 \mathrm{mmol})$, and $1,1-$ diphenylethylene 5a $(17.7 \mu \mathrm{L}, 0.1 \mathrm{mmol})$. The crude mixture was purified by column chromatography ( $\mathrm{SiO}_{2}$, pentane) to afford product $\mathbf{6 i}$ as a colorless oil $(25 \mathrm{mg}, 71 \%$ yield, average of two runs).

${ }^{1} \mathrm{H}$ NMR $\left(500 \mathrm{MHz}, \mathrm{CDCl}_{3}\right) \delta 7.31-7.26(\mathrm{~m}, 4 \mathrm{H}), 7.26-7.19(\mathrm{~m}, 6 \mathrm{H}), 5.84(\mathrm{dd}, J=10.5,2.2$ $\mathrm{Hz}, 1 \mathrm{H}$ ), 5.61 (ddt, $J=10.3,5.1,2.6 \mathrm{~Hz}, 1 \mathrm{H}), 3.44$ (t, $J=6.7 \mathrm{~Hz}, 2 \mathrm{H}), 3.21$ (ddd, $J=10.2$, 4.7, $2.3 \mathrm{~Hz}, 1 \mathrm{H}), 2.17-2.09(\mathrm{~m}, 2 \mathrm{H}), 1.92-1.84(\mathrm{~m}, 2 \mathrm{H}), 1.73-1.63(\mathrm{~m}, 3 \mathrm{H}), 1.57(\mathrm{dt}, J=8.9,3.1$ $\mathrm{Hz}, 2 \mathrm{H}), 1.36(\mathrm{dq}, J=9.4,7.5 \mathrm{~Hz}, 2 \mathrm{H}), 1.07-0.92(\mathrm{~m}, 3 \mathrm{H})$.

${ }^{13} \mathrm{C} \mathrm{NMR}\left(126 \mathrm{MHz}, \mathrm{CDCl}_{3}\right) \delta 146.3,144.1,129.8,129.4,128.6,127.4,126.9,125.8,125.6,54.3$, 45.1, 41.1, 39.1, 32.4, 27.6, 25.1, 24.8, 23.7, 22.5.

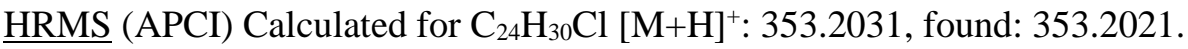

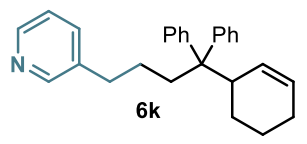

\section{3-(4-(cyclohex-2-en-1-yl)-4,4-diphenylbutyl)pyridine (6k)}

Prepared according to General Procedure B using the radical precursor 1n (43.4 $\mathrm{mg}, 0.1 \mathrm{mmol})$, cyclohexene $2 \mathrm{a}(101 \mu \mathrm{L}, 1.0 \mathrm{mmol})$, and $1,1-$ 
diphenylethylene 5a $(17.7 \mu \mathrm{L}, 0.1 \mathrm{mmol})$. The crude mixture was purified by preparative TLC $\left(\mathrm{SiO}_{2}, 90: 10\right.$ toluene/acetone) to afford product $\mathbf{6 k}$ as a colorless oil $(15.3 \mathrm{mg}, 42 \%$ yield, average of two runs).

${ }^{1} \mathrm{H} \mathrm{NMR}\left(500 \mathrm{MHz}, \mathrm{CDCl}_{3}\right) \delta 8.42(\mathrm{dd}, J=4.8,1.7 \mathrm{~Hz}, 1 \mathrm{H}), 8.36-8.32(\mathrm{~m}, 1 \mathrm{H}), 7.34-7.30$ $(\mathrm{m}, 1 \mathrm{H}), 7.27-7.24(\mathrm{~m}, 3 \mathrm{H}), 7.24-7.20(\mathrm{~m}, 2 \mathrm{H}), 7.20-7.15(\mathrm{~m}, 5 \mathrm{H}), 5.80(\mathrm{dp}, J=10.5,1.8$ $\mathrm{Hz}, 1 \mathrm{H}), 5.62-5.56(\mathrm{~m}, 1 \mathrm{H}), 3.22-3.13(\mathrm{~m}, 1 \mathrm{H}), 2.49(\mathrm{t}, J=7.5 \mathrm{~Hz}, 2 \mathrm{H}), 2.22-2.07(\mathrm{~m}, 2 \mathrm{H})$, $1.90-1.77(\mathrm{~m}, 2 \mathrm{H}), 1.72-1.64(\mathrm{~m}, 2 \mathrm{H}), 1.60-1.46(\mathrm{~m}, 2 \mathrm{H}), 1.41-1.29(\mathrm{~m}, 2 \mathrm{H}), 0.98-0.89$ $(\mathrm{m}, 1 \mathrm{H})$.

${ }^{13} \mathrm{C}$ NMR $\left(101 \mathrm{MHz}, \mathrm{CDCl}_{3}\right) \delta 149.9,147.2,146.0,143.8,137.3,135.7,129.7,129.4,129.2$, 128.6, 127.5, 126.9, 125.8, 125.7, 123.2, 54.2, 41.1, 38.4, 33.3, 25.6, 25.0, 24.7, 22.4.

HRMS (ESI) Calculated for $\mathrm{C}_{27} \mathrm{H}_{30} \mathrm{~N}[\mathrm{M}+\mathrm{H}]^{+}: 368.2373$, found: 368.2374 .

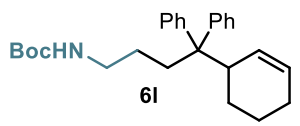

\section{Tert-butyl (4-(cyclohex-2-en-1-yl)-4,4-diphenylbutyl)carbamate (61)}

Prepared according to General Procedure B using the radical precursor 10 (47.2 $\mathrm{mg}, 0.1 \mathrm{mmol})$, cyclohexene $\mathbf{2 a}(101 \mu \mathrm{L}, 1.0 \mathrm{mmol})$, and $1,1-$ diphenylethylene 5a $(17.7 \mu \mathrm{L}, 0.1 \mathrm{mmol})$. The crude mixture was purified by column chromatography $\left(\mathrm{SiO}_{2}, 80: 20\right.$ hexanes/EtOAc) to afford product $\mathbf{6 1}$ as a colorless oil $(19.7 \mathrm{mg}$, $49 \%$ yield, average of two runs).

$\underline{{ }^{1} \mathrm{H} \mathrm{NMR}}\left(500 \mathrm{MHz}, \mathrm{CDCl}_{3}\right) \delta 7.30-7.19(\mathrm{~m}, 10 \mathrm{H}), 5.82(\mathrm{dt}, J=10.3,2.2 \mathrm{~Hz}, 1 \mathrm{H}), 5.65-5.57$ $(\mathrm{m}, 1 \mathrm{H}), 4.35(\mathrm{~s}, 1 \mathrm{H}), 3.23-3.16(\mathrm{~m}, 1 \mathrm{H}), 3.02(\mathrm{~d}, J=7.1 \mathrm{~Hz}, 2 \mathrm{H}), 2.19-2.07(\mathrm{~m}, 2 \mathrm{H}), 1.90-$ $1.83(\mathrm{~m}, 2 \mathrm{H}), 1.68(\mathrm{dtd}, J=13.6,7.5,6.8,4.2 \mathrm{~Hz}, 1 \mathrm{H}), 1.55(\mathrm{dtt}, J=13.4,7.7,3.3 \mathrm{~Hz}, 2 \mathrm{H}), 1.44$ (s, 9H), $1.20-1.09(\mathrm{~m}, 2 \mathrm{H}), 1.00-0.95(\mathrm{~m}, 1 \mathrm{H})$.

${ }^{13} \mathrm{C}$ NMR $\left(101 \mathrm{MHz}, \mathrm{CDCl}_{3}\right) \delta 155.8,146.0,143.8,129.7,129.3,129.2,128.7,127.5,126.9$, 125.8, 125.7, 54.0, 41.0, 36.3, 28.4, 25.0, 24.7, 22.4.

HRMS (ESI) Calculated for $\mathrm{C}_{27} \mathrm{H}_{35} \mathrm{NNaO}_{2}[\mathrm{M}+\mathrm{Na}]^{+}: 428.2560$, found: 428.2552 .

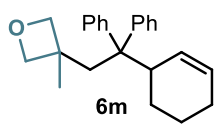

\section{3-(2-(cyclohex-2-en-1-yl)-2,2-diphenylethyl)-3-methyloxetane (6m)}

Prepared according to General Procedure B using the radical precursor 1p (47.9 $\mathrm{mg}, 0.12 \mathrm{mmol})$, cyclohexene $\mathbf{2 a}(101 \mu \mathrm{L}, 1.0 \mathrm{mmol})$, and 1,1-diphenylethylene $\mathbf{5 a}(17.7 \mu \mathrm{L}, 0.1 \mathrm{mmol})$. The crude mixture was purified by column chromatography $\left(\mathrm{SiO}_{2}, 20: 1\right.$ hexanes/ $\mathrm{Et}_{2} \mathrm{O}$ ) to afford product $\mathbf{6 m}$ as a white solid ( $28.2 \mathrm{mg}, 85 \%$ yield, average of two runs).

${ }^{1} \mathrm{H}$ NMR $\left(400 \mathrm{MHz}, \mathrm{CDCl}_{3}\right) \delta 7.37-7.15(\mathrm{~m}, 10 \mathrm{H}), 5.75(\mathrm{dp}, J=10.4,1.8 \mathrm{~Hz}, 1 \mathrm{H}), 5.54$ (ddt, $J=10.3,5.0,2.4 \mathrm{~Hz}, 1 \mathrm{H}), 3.85(\mathrm{~d}, J=5.7 \mathrm{~Hz}, 1 \mathrm{H}), 3.80(\mathrm{~d}, J=5.7 \mathrm{~Hz}, 1 \mathrm{H}), 3.65(\mathrm{~d}, J=5.7 \mathrm{~Hz}$, $1 \mathrm{H}), 3.62(\mathrm{~d}, J=5.7 \mathrm{~Hz}, 1 \mathrm{H}), 3.37-3.27(\mathrm{~m}, 1 \mathrm{H}), 2.69(\mathrm{~d}, J=14.6 \mathrm{~Hz}, 1 \mathrm{H}), 2.46(\mathrm{~d}, J=14.6$ $\mathrm{Hz}, 1 \mathrm{H}), 1.98-1.89(\mathrm{~m}, 1 \mathrm{H}), 1.88-1.77(\mathrm{~m}, 1 \mathrm{H}), 1.62(\mathrm{dddd}, J=12.8,6.2,4.5,2.5 \mathrm{~Hz}, 1 \mathrm{H})$, $1.58-1.48(\mathrm{~m}, 2 \mathrm{H}), 1.33(\mathrm{~s}, 3 \mathrm{H}), 0.98-0.81(\mathrm{~m}, 1 \mathrm{H})$.

${ }^{13} \mathrm{C} \mathrm{NMR}\left(101 \mathrm{MHz}, \mathrm{CDCl}_{3}\right) \delta 144.1,142.9,129.9,129.7,128.8,128.7,127.6,126.7,126.3$, 126.0, 83.9, 83.3, 54.2, 46.8, 42.9, 39.7, 25.4, 25.0, 24.9, 22.3.

HRMS (APCI) Calculated for $\mathrm{C}_{24} \mathrm{H}_{29} \mathrm{O}[\mathrm{M}+\mathrm{H}]^{+}: 333.2213$, found: 333.2207 .

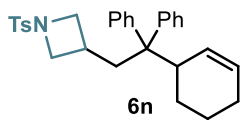

\section{3-(2-(cyclohex-2-en-1-yl)-2,2-diphenylethyl)-1-tosylazetidine (6n)}

Prepared according to General Procedure B using the radical precursor 1q (64.4 $\mathrm{mg}, 0.12 \mathrm{mmol})$, cyclohexene $2 \mathbf{a}(101 \mu \mathrm{L}, 1.0 \mathrm{mmol})$, and 1,1-diphenylethylene 
5a $(17.7 \mu \mathrm{L}, 0.1 \mathrm{mmol})$. The crude mixture was purified by column chromatography $\left(\mathrm{SiO}_{2}, 95: 5\right.$ hexanes/EtOAc) to afford product $\mathbf{6 n}$ as a colorless oil ( $28.9 \mathrm{mg}, 61 \%$ yield, average of two runs). ${ }^{1} \mathrm{H}$ NMR $\left(500 \mathrm{MHz}, \mathrm{CDCl}_{3}\right) \delta 7.58-7.54(\mathrm{~m}, 2 \mathrm{H}), 7.35-7.30(\mathrm{~m}, 2 \mathrm{H}), 7.25-7.19(\mathrm{~m}, 6 \mathrm{H})$, 7.05 (ddd, $J=7.7,3.5,1.6 \mathrm{~Hz}, 4 \mathrm{H}), 5.70(\mathrm{dp}, J=10.4,1.8 \mathrm{~Hz}, 1 \mathrm{H}), 5.56$ (ddq, $J=10.3,4.9,2.4$ $\mathrm{Hz}, 1 \mathrm{H}), 3.41(\mathrm{t}, J=8.1 \mathrm{~Hz}, 1 \mathrm{H}), 3.25(\mathrm{t}, J=8.1 \mathrm{~Hz}, 1 \mathrm{H}), 3.03(\mathrm{ddt}, J=6.9,4.6,2.5 \mathrm{~Hz}, 1 \mathrm{H})$, $2.83(\mathrm{dd}, J=8.1,7.0 \mathrm{~Hz}, 1 \mathrm{H}), 2.71(\mathrm{dd}, J=8.1,6.9 \mathrm{~Hz}, 1 \mathrm{H}), 2.45(\mathrm{~s}, 3 \mathrm{H}), 2.44-2.34(\mathrm{~m}, 1 \mathrm{H})$, $2.21-2.14(\mathrm{~m}, 2 \mathrm{H}), 2.00(\mathrm{dd}, J=14.2,7.3 \mathrm{~Hz}, 1 \mathrm{H}), 1.82(\mathrm{~d}, J=17.9 \mathrm{~Hz}, 1 \mathrm{H}), 1.76-1.67(\mathrm{~m}$, $1 \mathrm{H}), 1.55-1.41(\mathrm{~m}, 1 \mathrm{H}), 0.79$ (tdd, $J=12.3,10.2,3.4 \mathrm{~Hz}, 1 \mathrm{H})$.

${ }^{13} \mathrm{C} \mathrm{NMR}\left(126 \mathrm{MHz}, \mathrm{CDCl}_{3}\right) \delta 144.6,143.8,142.7,131.5,129.5,129.4,129.2,129.1,128.4$, $128.3,127.9,127.1,126.3,126.2,56.4,56.2,53.7,43.5,41.3,25.5,24.9,24.6,22.3,21.6$.

HRMS (ESI) Calculated for $\mathrm{C}_{30} \mathrm{H}_{33} \mathrm{NNaO}_{2} \mathrm{~S}[\mathrm{M}+\mathrm{Na}]^{+}: 494.2124$, found: 494.2117

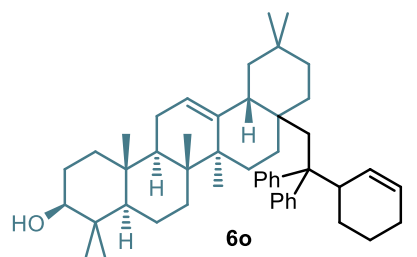

\section{(3S,4aR,6aR,6bS,12aR,14aR,14bR)-8a-(2-(cyclohex-2-en-1-yl)- 2,2-diphenylethyl)-4,4,6a,6b,11,11,14b-heptamethyl

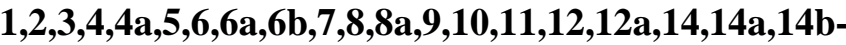 icosahydropicen-3-ol (6o)}

Prepared according to General Procedure B using the radical precursor $1 \mathbf{r}(74.0 \mathrm{mg}, 0.1 \mathrm{mmol})$, cyclohexene $2 \mathbf{a}(101 \mu \mathrm{L}, 1.0 \mathrm{mmol})$, and 1,1-diphenylethylene 5a $(17.7 \mu \mathrm{L}, 0.1 \mathrm{mmol})$. Multiple purifications by column chromatography resulted in poor separation from the simple decarboxylation product. The yield $(49 \%, 1.5: 1 \mathrm{dr}$, average of two runs) of 60 was inferred by ${ }^{1} \mathrm{H}$ NMR analysis of the crude reaction mixture using trichloroethylene as the internal standard. An analytical amount of $86 \%$ pure compound (containing $14 \%$ of the simple decarboxylation product as estimated by ${ }^{1} \mathrm{H}$ NMR) was isolated by preparative TLC (90:10 hexanes/EtOAc) to obtain a colorless oil.

${ }^{1} \mathrm{H}$ NMR $\left(500 \mathrm{MHz}, \mathrm{CDCl}_{3}\right)$ 1.5:1 mixture of diastereoiomers $\delta 7.46-7.13(\mathrm{~m}, 10 \mathrm{H}), 5.84(\mathrm{~d}, J$ $=10.6 \mathrm{~Hz}, 0.5 \mathrm{H}), 5.71(\mathrm{~d}, J=11.0 \mathrm{~Hz}, 0.5 \mathrm{H}), 5.55-5.45(\mathrm{~m}, 1 \mathrm{H}), 5.09-5.05(\mathrm{~m}, 0.5 \mathrm{H}), 4.96$ $(\mathrm{t}, J=3.6 \mathrm{~Hz}, 0.5 \mathrm{H}), 3.57-3.37(\mathrm{~m}, 1 \mathrm{H}), 3.28-3.20(\mathrm{~m}, 1 \mathrm{H}), 2.49(\mathrm{~d}, J=15.2 \mathrm{~Hz}, 0.5 \mathrm{H}), 2.43$ $-2.32(\mathrm{~m}, 1 \mathrm{H}), 2.21(\mathrm{~d}, J=11.5 \mathrm{~Hz}, 0.5 \mathrm{H}), 2.07-1.93(\mathrm{~m}, 2 \mathrm{H}), 1.92-1.73(\mathrm{~m}, 5 \mathrm{H}), 1.69-1.53$ $(\mathrm{m}, 14 \mathrm{H}), 1.44(\mathrm{qd}, J=12.5,3.2 \mathrm{~Hz}, 5 \mathrm{H}), 1.08(\mathrm{~d}, J=4.6 \mathrm{~Hz}, 2 \mathrm{H}), 1.04-1.00(\mathrm{~m}, 6 \mathrm{H}), 0.98(\mathrm{~d}$, $J=4.1 \mathrm{~Hz}, 4 \mathrm{H}), 0.95-0.89(\mathrm{~m}, 4 \mathrm{H}), 0.81(\mathrm{~d}, J=2.3 \mathrm{~Hz}, 3 \mathrm{H}), 0.75(\mathrm{~d}, J=1.6 \mathrm{~Hz}, 4 \mathrm{H}), 0.54(\mathrm{~d}$, $J=6.2 \mathrm{~Hz}, 2 \mathrm{H})$.

${ }^{13} \mathrm{C} \mathrm{NMR}\left(126 \mathrm{MHz}, \mathrm{CDCl}_{3}\right)$ 1.5:1 mixture of diastereoiomers $\delta 145.4,145.1,129.7,129.5$, 128.3, 127.1, 127.0, 126.2, 126.1, 125.6, 125.5, 125.4, 122.2, 122.0, 79.0, 55.2, 55.1, 54.6, 54.2, 47.7, 47.6, 47.5, 46.7, 46.6, 44.9, 44.1, 43.9, 41.8, 40.9, 40.8, 39.9, 38.7, 38.5, 38.4, 37.2, 37.1, $36.9,36.8,35.7,34.4,34.3,33.6,33.3,33.0,32.3,32.1,31.1,30.9,30.6,30.3,30.2,29.2$, 28.0, $28.0,27.2,26.9,26.9,26.2,26.1,25.4,25.0,23.9,23.8,23.7,23.6,23.6,22.7,22.2,18.3,16.9$, $16.8,15.6,15.5,15.5,15.5,15.3$.

HRMS (APCI) Calculated for $\mathrm{C}_{49} \mathrm{H}_{69} \mathrm{O}[\mathrm{M}+\mathrm{H}]^{+}:$673.5343, found: 673.5337.

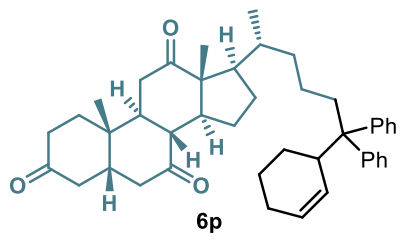

(5S,8R,9S,10S,13R,14S,17R)-17-(6-(cyclohex-2-en-1-yl) 6,6diphenylhexan-2-yl)-10,13-dimethyldodecahydro-3Hcyclopenta[a]phenanthrene-3,7,12(2H,4H)-trione (6p)

Prepared according to General Procedure B using the radical precursor $1 \mathrm{~s}$ (68.5 mg, $0.1 \mathrm{mmol})$, cyclohexene 2a $(101 \mu \mathrm{L}, 1.0$ mmol), and 1,1-diphenylethylene $\mathbf{5 a}(17.7 \mu \mathrm{L}, 0.1 \mathrm{mmol})$. The crude mixture was purified by 
column chromatography $\left(\mathrm{SiO}_{2}, 90: 10\right.$ toluene/EtOAc) to afford product $\mathbf{6 p}$ as a white solid (40.7 $\mathrm{mg}, 66 \%$ yield, 1.1:1 d.r., average of two runs).

${ }^{1} \mathrm{H}$ NMR $\left(500 \mathrm{MHz}, \mathrm{CDCl}_{3}\right)$ 1.1:1 mixture of diastereoiomers $\delta 7.31-7.17(\mathrm{~m}, 10 \mathrm{H}), 5.83(\mathrm{t}, J$ $=8.7 \mathrm{~Hz}, 1 \mathrm{H}), 5.63-5.55(\mathrm{~m}, 1 \mathrm{H}), 3.20(\mathrm{~s}, 1 \mathrm{H}), 2.96-2.79(\mathrm{~m}, 3 \mathrm{H}), 2.38-2.21(\mathrm{~m}, 6 \mathrm{H}), 2.17$ $-2.02(\mathrm{~m}, 5 \mathrm{H}), 2.01-1.79(\mathrm{~m}, 6 \mathrm{H}), 1.71-1.52(\mathrm{~m}, 5 \mathrm{H}), 1.25(\mathrm{~d}, J=24.8 \mathrm{~Hz}, 6 \mathrm{H}), 1.16-1.06$ $(\mathrm{m}, 2 \mathrm{H}), 1.03(\mathrm{~d}, J=2.4 \mathrm{~Hz}, 3 \mathrm{H}), 0.89-0.74(\mathrm{~m}, 2 \mathrm{H}), 0.66(\mathrm{dd}, J=9.3,6.5 \mathrm{~Hz}, 3 \mathrm{H})$.

${ }^{13} \mathrm{C}$ NMR $\left(126 \mathrm{MHz}, \mathrm{CDCl}_{3}\right)$ 1.1:1 mixture of diastereoiomers $\delta$ 212.0, 209.0, 208.8, 146.4, 144.6, 144.1, 129.8, 129.8, 129.5, 129.5, 129.4, 128.5, 128.4, 127.3, 126.9, 126.8, 125.6, 125.6, $125.4,56.9,54.5,54.3,51.7,49.0,46.8,46.0,46.0,45.5,45.0,42.8,41.4,40.7,39.7,39.5,38.6$, $36.5,36.2,36.0,35.9,35.8,35.3,29.7,29.2,27.7,27.7,25.1,25.0,24.8,24.7,22.5,22.4,21.9$, 21.4, 21.3, 18.9, 11.8 .

$\underline{\text { HRMS }}$ (ESI) Calculated for $\mathrm{C}_{43} \mathrm{H}_{54} \mathrm{NaO}_{3}[\mathrm{M}+\mathrm{Na}]^{+}: 641.3965$, found: 641.3964 .

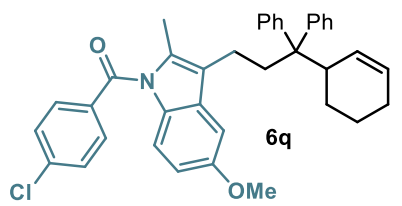

(4-chlorophenyl)(3-(3-(cyclohex-2-en-1-yl)-3,3-diphenylpropyl)5-methoxy-2-methyl-1H-indol-1-yl)methanone (6q)

Prepared according to General Procedure B using the radical precursor 1e $(64.1 \mathrm{mg}, 0.1 \mathrm{mmol})$, cyclohexene $2 \mathbf{a}(101 \mu \mathrm{L}, 1.0$ mmol), 1,1-diphenylethylene 5a (35.3 $\mu \mathrm{L}, 0.2 \mathrm{mmol})$, and catalyst $\mathbf{C 5}(7.6 \mathrm{mg}, 0.2 \mathrm{mmol})$. The crude mixture was purified by column chromatography $\left(\mathrm{SiO}_{2}, 98: 2\right.$ hexanes/EtOAc) to afford product $\mathbf{6 q}$ as a yellowish oil (20.8 $\mathrm{mg}, 36 \%$ yield, average of two runs).

${ }^{1} \mathrm{H}$ NMR $\left(500 \mathrm{MHz}, \mathrm{CDCl}_{3}\right) \delta 7.66-7.63(\mathrm{~m}, 2 \mathrm{H}), 7.49-7.45(\mathrm{~m}, 2 \mathrm{H}), 7.38-7.29(\mathrm{~m}, 8 \mathrm{H})$, $7.28-7.22(\mathrm{~m}, 2 \mathrm{H}), 6.86(\mathrm{~d}, J=9.0 \mathrm{~Hz}, 1 \mathrm{H}), 6.63(\mathrm{dd}, J=9.0,2.6 \mathrm{~Hz}, 1 \mathrm{H}), 6.47(\mathrm{~d}, J=2.5 \mathrm{~Hz}$, $1 \mathrm{H}), 5.95(\mathrm{~d}, J=10.4 \mathrm{~Hz}, 1 \mathrm{H}), 5.67$ (ddd, $J=10.4,4.8,2.5 \mathrm{~Hz}, 1 \mathrm{H}), 3.78(\mathrm{~s}, 3 \mathrm{H}), 3.38(\mathrm{~s}, 1 \mathrm{H})$, $2.45-2.37(\mathrm{~m}, 1 \mathrm{H}), 2.36-2.25(\mathrm{~m}, 3 \mathrm{H}), 2.18(\mathrm{~s}, 3 \mathrm{H}), 2.01-1.87(\mathrm{~m}, 2 \mathrm{H}), 1.77-1.66(\mathrm{~m}, 1 \mathrm{H})$, $1.34(\mathrm{~d}, J=24.8 \mathrm{~Hz}, 1 \mathrm{H}), 1.15-1.03(\mathrm{~m}, 1 \mathrm{H}), 0.96-0.84(\mathrm{~m}, 1 \mathrm{H})$.

${ }^{13} \mathrm{C} \mathrm{NMR}\left(101 \mathrm{MHz}, \mathrm{CDCl}_{3}\right) \delta 168.3,155.8,146.2,144.0,138.9,134.3,133.5,131.0,131.0$, 130.9, 129.9, 129.6, 129.1, 129.0, 129.0, 127.7, 127.1, 126.0, 125.9, 120.2, 115.1, 111.4, 100.6, $55.7,54.5,40.3,39.3,25.1,24.9,22.5,18.9,13.1$.

HRMS (ESI) Calculated for $\mathrm{C}_{38} \mathrm{H}_{36} \mathrm{ClNNaO}_{2}[\mathrm{M}+\mathrm{Na}]^{+}:$596.2327, found: 596.2336.

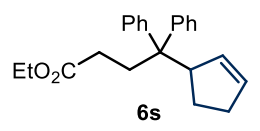

\section{Ethyl 4-(cyclopent-2-en-1-yl)-4,4-diphenylbutanoate (6s)}

Prepared according to General Procedure B using the radical precursor $4 \mathbf{b}$ (48.1 $\mathrm{mg}, 0.1 \mathrm{mmol})$, cyclopentene $2 \mathbf{c}(110 \mu \mathrm{L}, 1.0 \mathrm{mmol})$, and 1,1-diphenylethylene 5a $(17.7 \mu \mathrm{L}, 0.1 \mathrm{mmol})$. The crude mixture was purified by column chromatography $\left(\mathrm{SiO}_{2}, 70: 30\right.$ hexanes/toluene) to afford product $\mathbf{6 s}$ as a colorless oil ( $25.0 \mathrm{mg}, 75 \%$ yield, average of two runs). ${ }^{1} \mathrm{H}$ NMR $\left(500 \mathrm{MHz}, \mathrm{CDCl}_{3}\right) \delta 7.29-7.19(\mathrm{~m}, 10 \mathrm{H}), 5.83(\mathrm{dq}, J=6.0,2.0 \mathrm{~Hz}, 1 \mathrm{H}), 5.64(\mathrm{dq}, J=$ $5.8,2.3 \mathrm{~Hz}, 1 \mathrm{H}), 4.07$ (q, $J=7.2 \mathrm{~Hz}, 2 \mathrm{H}), 3.76$ (ddt, $J=8.5,6.0,2.5 \mathrm{~Hz}, 1 \mathrm{H}), 2.53$ (ddd, $J=13.9$, $11.7,4.8 \mathrm{~Hz}, 1 \mathrm{H}), 2.39$ (ddd, $J=13.8,11.6,5.2 \mathrm{~Hz}, 1 \mathrm{H}), 2.12$ (ddd, $J=16.4,11.7,4.8 \mathrm{~Hz}, 1 \mathrm{H}$ ), $2.07-1.98(\mathrm{~m}, 3 \mathrm{H}), 1.54-1.46(\mathrm{~m}, 1 \mathrm{H}), 1.22(\mathrm{t}, J=7.2 \mathrm{~Hz}, 3 \mathrm{H}), 0.94-0.84(\mathrm{~m}, 1 \mathrm{H})$.

${ }^{13} \mathrm{C} \mathrm{NMR}\left(126 \mathrm{MHz}, \mathrm{CDCl}_{3}\right) \delta 173.9,145.9,144.1,133.3,131.8,129.8,129.2,127.4,127.2$, $125.9,125.8,60.3,53.5,51.3,34.8,31.6,30.0,25.7,14.1$.

HRMS (ESI) Calculated for $\mathrm{C}_{23} \mathrm{H}_{26} \mathrm{NaO}_{2}[\mathrm{M}+\mathrm{Na}]^{+}: 357.1825$, found: 357.1818 . 


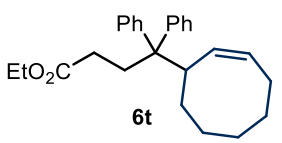

Ethyl (Z)-4-(cyclooct-2-en-1-yl)-4,4-diphenylbutanoate (6t)

Prepared according to General Procedure B using the radical precursor $\mathbf{4 b}$ (48.1 $\mathrm{mg}, 0.1 \mathrm{mmol})$, Z-cyclooctene $2 \mathrm{e}(130 \mu \mathrm{L}, 1.0 \mathrm{mmol})$, and 1,1diphenylethylene 5a $(17.7 \mu \mathrm{L}, 0.1 \mathrm{mmol})$. Multiple purifications by column chromatography resulted in poor separation from several unidentified byproducts. The yield (39\%, average of two runs) of $6 \mathbf{t}$ was inferred by ${ }^{1} \mathrm{H}$ NMR analysis of the crude reaction mixture using trichloroethylene as the internal standard. An analytical amount of pure compound was isolated by preparative TLC (50:50 hexanes/toluene) to obtain a colorless oil.

${ }^{1} \mathrm{H} \mathrm{NMR}\left(400 \mathrm{MHz}, \mathrm{CDCl}_{3}\right) \delta 7.32-7.25(\mathrm{~m}, 4 \mathrm{H}), 7.25-7.18(\mathrm{~m}, 6 \mathrm{H}), 5.66(\mathrm{td}, J=9.6,7.0 \mathrm{~Hz}$, $1 \mathrm{H}), 5.27$ (ddd, $J=10.8,9.5,1.4 \mathrm{~Hz}, 1 \mathrm{H}), 4.01(\mathrm{q}, J=7.1 \mathrm{~Hz}, 2 \mathrm{H}), 3.50-3.40(\mathrm{~m}, 1 \mathrm{H}), 2.38(\mathrm{dd}$, $J=9.0,7.7 \mathrm{~Hz}, 2 \mathrm{H}), 2.14(\mathrm{ddd}, J=13.4,7.3,3.7 \mathrm{~Hz}, 1 \mathrm{H}), 1.93(\mathrm{td}, J=7.9,6.3 \mathrm{~Hz}, 2 \mathrm{H}), 1.90-$ $1.81(\mathrm{~m}, 1 \mathrm{H}), 1.74(\mathrm{ddd}, J=13.6,7.5,3.7 \mathrm{~Hz}, 1 \mathrm{H}), 1.66-1.46(\mathrm{~m}, 4 \mathrm{H}), 1.45-1.29(\mathrm{~m}, 2 \mathrm{H}), 1.17$ (t, $J=7.1 \mathrm{~Hz}, 3 \mathrm{H}), 0.76(\mathrm{tt}, J=12.7,5.0 \mathrm{~Hz}, 1 \mathrm{H})$.

${ }^{13} \mathrm{C}$ NMR $\left(101 \mathrm{MHz}, \mathrm{CDCl}_{3}\right) \delta 173.8,145.3,144.0,130.6,130.3,129.5,127.6,127.1,126.1$, 125.9, 60.2, 53.1, 39.6, 35.7, 31.1, 29.8, 29.6, 26.7, 26.7, 25.9, 14.1.

HRMS (ESI) Calculated for $\mathrm{C}_{26} \mathrm{H}_{32} \mathrm{NaO}_{2}[\mathrm{M}+\mathrm{Na}]^{+}: 399.22875$ found: 399.2287 .

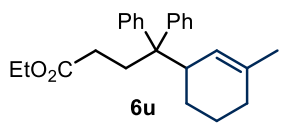

Ethyl 4-(3-methylcyclohex-2-en-1-yl)-4,4-diphenylbutanoate (6u)

Prepared according to General Procedure B using the radical precursor $\mathbf{4 b}$ (48.1 mg, $0.1 \mathrm{mmol}), 1$-methylcyclohexene $2 \mathrm{~d}(118 \mu \mathrm{L}, 1.0 \mathrm{mmol})$, and 1,1-

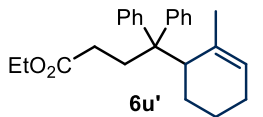
diphenylethylene $\mathbf{5 a}(17.7 \mu \mathrm{L}, 0.1 \mathrm{mmol})$. The crude mixture was purified by column chromatography $\left(\mathrm{SiO}_{2}, 70: 30\right.$ hexanes/toluene) to afford product $\mathbf{6 u}$ as a colorless oil ( $24.3 \mathrm{mg}, 67 \%$ yield, 5:1 r.r., average of two runs).

${ }^{1} \mathrm{H} \mathrm{NMR}\left(400 \mathrm{MHz}, \mathrm{CDCl}_{3}\right)$ 5:1 mixture of regioisomers $\delta 7.33-7.15(\mathrm{~m}, 12 \mathrm{H}), 5.47$ (brs, $1 \mathrm{H}), 5.46-5.42(\mathrm{~m}, 0.2 \mathrm{H}), 4.09-3.99(\mathrm{~m}, 2.4 \mathrm{H}), 3.19-3.08(\mathrm{~m}, 1.2 \mathrm{H}), 2.55$ (ddd, $J=$ $13.9,11.8,4.5 \mathrm{~Hz}, 1 \mathrm{H}), 2.48-2.41(\mathrm{~m}, 0.2 \mathrm{H}), 2.36(\mathrm{ddd}, J=14.0,11.8,5.3 \mathrm{~Hz}, 1 \mathrm{H}), 2.24(\mathrm{td}, J$ $=15.3,14.6,3.2 \mathrm{~Hz}, 0.2 \mathrm{H}), 2.06-1.98(\mathrm{~m}, 1.2 \mathrm{H}), 1.96-1.90(\mathrm{~m}, 1 \mathrm{H}), 1.90-1.85(\mathrm{~m}, 0.2 \mathrm{H})$, $1.86-1.76(\mathrm{~m}, 1.2 \mathrm{H}), 1.70(\mathrm{dd}, J=15.9,5.5 \mathrm{~Hz}, 1.2 \mathrm{H}), 1.57(\mathrm{~s}, 0.8 \mathrm{H}), 1.52(\mathrm{dd}, J=2.3,1.3 \mathrm{~Hz}$, $3 \mathrm{H}), 1.23-1.15(\mathrm{~m}, 3.6 \mathrm{H}), 0.85-0.79(\mathrm{~m}, 1.2 \mathrm{H})$.

${ }^{13} \mathrm{C} \mathrm{NMR}\left(101 \mathrm{MHz}, \mathrm{CDCl}_{3}\right)$ major regioisomer $\delta 173.9,145.5,143.0,135.8,129.7,129.3$, 127.6, 127.6, 126.9, 125.8, 125.8, 123.0, 60.2, 53.9, 41.7, 33.9, 30.1, 29.8, 24.4, 23.9, 22.5, 14.1.

HRMS (ESI) Calculated for $\mathrm{C}_{25} \mathrm{H}_{30} \mathrm{NaO}_{2}[\mathrm{M}+\mathrm{Na}]^{+}: 385.2138$ found: 385.2139 .

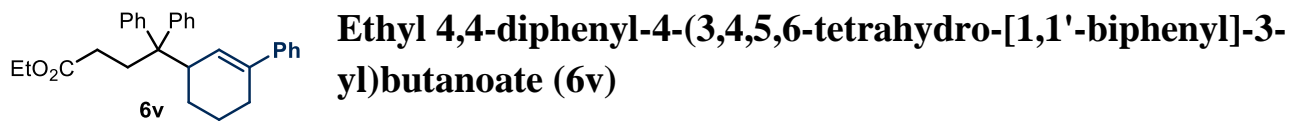

Prepared according to General Procedure B using the radical precursor $\mathbf{4 b}$ (48.1 mg, $0.1 \mathrm{mmol}), 1$-phenylcyclohexene $\mathbf{2 f}(160 \mu \mathrm{L}, 1.0 \mathrm{mmol})$, and 1,1-diphenylethylene $\mathbf{5 a}$ $(17.7 \mu \mathrm{L}, 0.1 \mathrm{mmol})$. The crude mixture was purified by column chromatography $\left(\mathrm{SiO}_{2}, 70: 30\right.$ hexanes/toluene) to afford product $6 \mathbf{v}$ as a colorless oil (22.0 mg, 52\% yield, average of two runs). ${ }^{1} \mathrm{H} \mathrm{NMR}\left(400 \mathrm{MHz}, \mathrm{CDCl}_{3}\right) \delta 7.32-7.29(\mathrm{~m}, 5 \mathrm{H}), 7.28-7.17(\mathrm{~m}, 9 \mathrm{H}), 6.17(\mathrm{dt}, J=2.6,1.4 \mathrm{~Hz}$, $1 \mathrm{H}), 4.08(\mathrm{q}, J=7.2 \mathrm{~Hz}, 2 \mathrm{H}), 3.37(\mathrm{~d}, J=9.5 \mathrm{~Hz}, 1 \mathrm{H}), 2.62(\mathrm{ddd}, J=14.0,11.6,4.8 \mathrm{~Hz}, 1 \mathrm{H})$, 2.49 (ddd, $J=14.0,11.5,5.6 \mathrm{~Hz}, 1 \mathrm{H}), 2.38-2.29(\mathrm{~m}, 1 \mathrm{H}), 2.12-1.93(\mathrm{~m}, 5 \mathrm{H}), 1.81-1.74(\mathrm{~m}$, $1 \mathrm{H}), 1.72-1.64(\mathrm{~m}, 1 \mathrm{H}), 1.23(\mathrm{t}, J=7.1 \mathrm{~Hz}, 3 \mathrm{H}), 1.03$ (tdd, $J=12.7,10.2,3.3 \mathrm{~Hz}, 1 \mathrm{H})$. 
${ }^{13} \mathrm{C}$ NMR $\left(101 \mathrm{MHz}, \mathrm{CDCl}_{3}\right) \delta 173.8,145.1,142.9,142.8,138.4,129.7,129.3,128.1,127.8$, 126.7, 126.6, 126.1, 126.0, 125.3, 60.3, 54.2, 42.1, 34.1, 30.1, 27.4, 24.2, 22.7, 14.1.

$\underline{\text { HRMS }}$ (ESI) Calculated for $\mathrm{C}_{30} \mathrm{H}_{32} \mathrm{NaO}_{2}[\mathrm{M}+\mathrm{Na}]^{+}: 447.2295$ found: 447.2293.

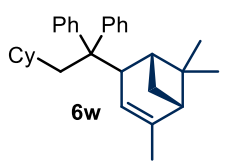

\section{(1S,5R)-4-(2-cyclohexyl-1,1-diphenylethyl)-2,6,6- trimethylbicyclo[3.1.1]hept-2-ene (6w)}

Prepared according to General Procedure B using the radical precursor $\mathbf{1 h}$ (49.3 mg, $0.12 \mathrm{mmol}), \alpha$-pinene $\mathbf{2 g}(158 \mu \mathrm{L}, 1.0 \mathrm{mmol})$, and 1,1-diphenylethylene $\mathbf{5 a}(17.7 \mu \mathrm{L}, 0.1$ mmol). The crude mixture was purified by column chromatography $\left(\mathrm{SiO}_{2}\right.$, cyclohexane) to afford product $6 \mathrm{w}$ as a colorless oil (19.4 $\mathrm{mg}, 49 \%$ yield, $>10: 1 \mathrm{~d} . r$., average of two runs).

$\underline{{ }^{1} \mathrm{H} \mathrm{NMR}}\left(500 \mathrm{MHz}, \mathrm{CDCl}_{3}\right) \delta 7.28-7.19(\mathrm{~m}, 10 \mathrm{H}), 5.49(\mathrm{p}, J=1.8 \mathrm{~Hz}, 1 \mathrm{H}), 3.33(\mathrm{q}, J=2.3 \mathrm{~Hz}$, $1 \mathrm{H}), 2.21(\mathrm{tt}, J=5.8,1.9 \mathrm{~Hz}, 1 \mathrm{H}), 2.14(\mathrm{dd}, J=14.1,3.5 \mathrm{~Hz}, 1 \mathrm{H}), 1.87(\mathrm{dd}, J=14.1,5.2 \mathrm{~Hz}, 1 \mathrm{H})$, $1.66(\mathrm{td}, J=5.4,1.3 \mathrm{~Hz}, 1 \mathrm{H}), 1.59(\mathrm{dd}, J=2.5,1.6 \mathrm{~Hz}, 3 \mathrm{H}), 1.55-1.41(\mathrm{~m}, 6 \mathrm{H}), 1.23(\mathrm{~s}, 3 \mathrm{H})$, $1.03(\mathrm{td}, J=11.3,4.4 \mathrm{~Hz}, 3 \mathrm{H}), 0.97(\mathrm{~s}, 3 \mathrm{H}), 0.95-0.84(\mathrm{~m}, 3 \mathrm{H}), 0.72-0.63(\mathrm{~m}, 1 \mathrm{H})$.

${ }^{13} \mathrm{C} \mathrm{NMR}\left(101 \mathrm{MHz}, \mathrm{CDCl}_{3}\right) \delta 147.0,145.5,129.6,129.4,127.2,126.9,125.5,125.4,116.8,54.0$, 46.9, 46.2, 44.8, 42.0, 41.3, 35.6, 35.1, 33.6, 26.6, 26.4, 26.3, 25.4, 23.2, 20.7.

$\underline{\text { HRMS }}$ (APCI) Calculated for $\mathrm{C}_{30} \mathrm{H}_{39}[\mathrm{M}+\mathrm{H}]^{+}: 399.3046$ found: 399.3036.

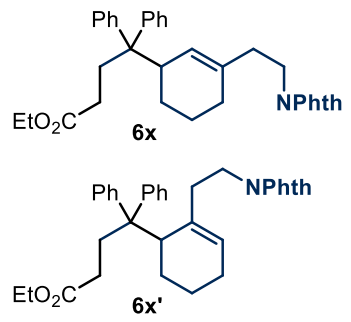

\section{ethyl 4-(3-(2-(1,3-dioxoisoindolin-2-yl)ethyl)cyclohex-2-en-1-yl)-4,4- diphenylbutanoate $(6 \mathbf{x})$}

Prepared according to General Procedure B using the radical precursor 4b (48.1 mg, $0.1 \mathrm{mmol}$ ), 2-(2-(cyclohex-1-en-1-yl)ethyl)isoindoline-1,3dione $\mathbf{2 h}(128 \mathrm{mg}, 5.0 \mathrm{mmol})$, and 1,1-diphenylethylene $\mathbf{5 a}(17.7 \mu \mathrm{L}, 0.1$ mmol). The crude mixture was purified by column chromatography $\left(\mathrm{SiO}_{2}, 90: 10\right.$ hexanes/EtOAc) to afford product $\mathbf{6} \mathbf{x}$ as a colorless oil $(32.3$ $\mathrm{mg}, 62 \%$ yield, 5:1 r.r., average of two runs). When using catalyst $\mathbf{C 7}, \mathbf{6} \mathbf{x}$ was obtained as a colorless oil ( $25 \mathrm{mg}, 48 \%$ yield, 10:1 r.r.).

${ }^{1} \mathrm{H}$ NMR $\left(500 \mathrm{MHz}, \mathrm{CDCl}_{3}\right) 5: 1$ mixture of regioisomers $\delta 7.88(\mathrm{dd}, J=5.4,3.1 \mathrm{~Hz}, 2 \mathrm{H}), 7.79$ $(\mathrm{td}, J=5.4,2.3 \mathrm{~Hz}, 2 \mathrm{H}), 7.22-7.11(\mathrm{~m}, 6 \mathrm{H}), 7.11-7.06(\mathrm{~m}, 2 \mathrm{H}), 7.03-6.98(\mathrm{~m}, 2 \mathrm{H}), 5.47(\mathrm{~s}$, $1 \mathrm{H}), 5.26(\mathrm{~m}, 0.2 \mathrm{H}), 4.04(\mathrm{q}, J=7.2 \mathrm{~Hz}, 2 \mathrm{H}), 3.70-3.63(\mathrm{~m}, 1 \mathrm{H}), 3.60(\mathrm{ddd}, J=13.4,7.8,5.3$ $\mathrm{Hz}, 1 \mathrm{H}), 3.02(\mathrm{~d}, J=9.2 \mathrm{~Hz}, 1 \mathrm{H}), 2.46(\mathrm{ddd}, J=14.0,11.2,5.2 \mathrm{~Hz}, 1 \mathrm{H}), 2.35(\mathrm{ddd}, J=13.9$, $11.1,5.9 \mathrm{~Hz}, 1 \mathrm{H}), 2.20(\mathrm{dt}, J=15.0,7.6 \mathrm{~Hz}, 1 \mathrm{H}), 2.13(\mathrm{dt}, J=12.8,6.2 \mathrm{~Hz}, 1 \mathrm{H}), 1.97-1.90(\mathrm{~m}$, $2 \mathrm{H}), 1.86(\mathrm{dt}, J=13.5,4.1 \mathrm{~Hz}, 2 \mathrm{H}), 1.70-1.61(\mathrm{~m}, 2 \mathrm{H}), 1.58-1.49(\mathrm{~m}, 1 \mathrm{H}), 1.20(\mathrm{t}, J=7.2 \mathrm{~Hz}$, $3 \mathrm{H}), 0.90-0.81(\mathrm{~m}, 1 \mathrm{H})$.

${ }^{13} \mathrm{C} \mathrm{NMR}\left(126 \mathrm{MHz}, \mathrm{CDCl}_{3}\right)$ major regioisomer $\delta 173.8,168.1,145.1,142.8,135.9,133.8,132.2$, 129.7, 129.1, 127.6, 126.9, 126.0, 125.9, 125.8, 123.1, 60.2, 53.7, 41.3, 36.9, 36.4, 33.9, 29.9, 27.6, 24.2, 22.5, 14.1 .

$\underline{\text { HRMS }}$ (ESI) Calculated for $\mathrm{C}_{34} \mathrm{H}_{35} \mathrm{NNaO}_{4}[\mathrm{M}+\mathrm{Na}]^{+}: 544.2458$ found: 544.2469. 


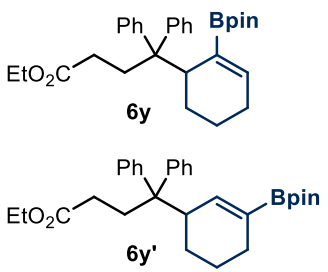

ethyl 4,4-diphenyl-4-(2-(4,4,5,5-tetramethyl-1,3,2-dioxaborolan-2yl)cyclohex-2-en-1-yl)butanoate (6y)

Prepared according to General Procedure B using the radical precursor $\mathbf{4 b}$ (48.1 mg, $0.1 \mathrm{mmol}$ ), 2-(cyclohex-1-en-1-yl)-4,4,5,5-tetramethyl-1,3,2dioxaborolane $2 \mathbf{i}(110 \mu \mathrm{L}, 0.5 \mathrm{mmol})$, and 1,1-diphenylethylene $\mathbf{5 a}(17.7$ $\mu \mathrm{L}, \quad 0.1 \mathrm{mmol})$. The crude mixture was purified by column chromatography $\left(\mathrm{SiO}_{2}, 50: 50\right.$ hexanes/toluene) to afford product $6 \mathbf{y}$ as a colorless oil $(21.3 \mathrm{mg}$, $45 \%$ yield, 1.2:1 r.r., average of two runs). When using catalyst $\mathbf{C 6}$, the yield (50\% yield, 1:4 r.r.) of $\mathbf{6 y}{ }^{\prime}$ was inferred by ${ }^{1} \mathrm{H}$ NMR analysis of the crude reaction mixture using trichloroethylene as the internal standard.

${ }^{1} \mathrm{H} \mathrm{NMR}\left(500 \mathrm{MHz}, \mathrm{CDCl}_{3}\right)$ 1.2:1 mixture of regioisomers $\delta 7.43-7.39(\mathrm{~m}, 1 \mathrm{H}), 7.36-7.29(\mathrm{~m}$, $3 \mathrm{H}), 7.27-7.20(\mathrm{~m}, 6 \mathrm{H}), 6.68(\mathrm{~d}, J=2.6 \mathrm{~Hz}, 0.5 \mathrm{H}), 6.61(\mathrm{ddd}, J=4.9,3.4,1.2 \mathrm{~Hz}, 0.5 \mathrm{H}), 4.06$ (q, $J=7.1 \mathrm{~Hz}, \mathrm{H}), 3.99(\mathrm{qd}, J=7.1,0.9 \mathrm{~Hz}, 1 \mathrm{H}), 3.60-3.55(\mathrm{~m}, 1 \mathrm{H}), 2.56-2.44(\mathrm{~m}, 1 \mathrm{H}), 2.43$ $-2.32(\mathrm{~m}, 1 \mathrm{H}), 2.20$ (ddd, $J=15.1,11.6,5.3 \mathrm{~Hz}, 1 \mathrm{H}), 1.98$ (dd, $J=9.3,7.4 \mathrm{~Hz}, 1 \mathrm{H}), 1.75$ (qd, $J$ $=6.1,3.0 \mathrm{~Hz}, 1 \mathrm{H}), 1.70-1.63(\mathrm{~m}, 1 \mathrm{H}), 1.28(\mathrm{~d}, J=2.2 \mathrm{~Hz}, 1 \mathrm{H}), 1.26(\mathrm{~s}, 4 \mathrm{H}), 1.24(\mathrm{~s}, 3 \mathrm{H}), 1.21$ $(\mathrm{d}, J=2.0 \mathrm{~Hz}, 3 \mathrm{H}), 1.20-1.19(\mathrm{~m}, 3 \mathrm{H}), 1.17(\mathrm{~d}, J=7.1 \mathrm{~Hz}, 1 \mathrm{H}), 1.07-0.99(\mathrm{~m}, 1 \mathrm{H}), 0.95-$ $0.82(\mathrm{~m}, 2 \mathrm{H}), 0.73-0.64(\mathrm{~m}, 0.5 \mathrm{H})$.

${ }^{13} \mathrm{C} \mathrm{NMR}\left(101 \mathrm{MHz}, \mathrm{CDCl}_{3}\right)$ 1.2:1 mixture of regioisomers $\delta 174.0,173.9,146.9,143.8,143.3$, 143.0, 130.6, 130.4, 129.8, 129.4, 127.8, 127.4, 127.0, 126.0, 125.9, 83.2, 82.9, 60.3, 60.0, 56.7, 53.9, 41.9, 40.1, 36.3, 33.9, 30.7, 30.0, 26.3, 25.1, 25.0, 24.9, 24.4, 24.3, 22.5, 19.4, 14.1 .

HRMS (ESI) Calculated for $\mathrm{C}_{30} \mathrm{H}_{39} \mathrm{NaO}_{4}{ }^{10} \mathrm{~B}[\mathrm{M}+\mathrm{Na}]^{+}: 496.2870$ found: 496.2864 .

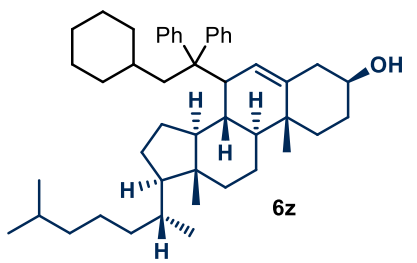

\section{(3S,8S,9S,10R,13R,14S,17R)-7-(2-cyclohexyl-1,1- diphenylethyl)-10,13-dimethyl-17-((R)-6-methylheptan-2-yl)-

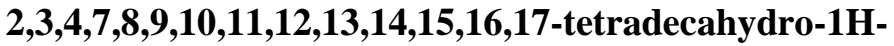 cyclopenta[a]phenanthren-3-ol (6z)}

Prepared according to General Procedure B using the radical precursor $\mathbf{1 h}$ (49.3 mg, $0.12 \mathrm{mmol}$ ), cholesterol $\mathbf{2 j}$ ( $193 \mathrm{~mL}, 0.5 \mathrm{mmol}$ ), and 1,1-diphenylethylene 5a $(17.7 \mu \mathrm{L}, 0.1 \mathrm{mmol})$. The crude mixture was purified by column chromatography $\left(\mathrm{SiO}_{2}, 95: 5\right.$ hexanesEtOAc) to afford product $\mathbf{6 y}$ as a white solid. The yield (48\% yield, 5:1 d.r., average of two runs) of $\mathbf{6 z}$ was inferred by ${ }^{1} \mathrm{H}$ NMR analysis of the crude reaction mixture using trichloroethylene as the internal standard.

${ }^{1} \mathrm{H} \mathrm{NMR}\left(500 \mathrm{MHz}, \mathrm{CDCl}_{3}\right)$ 5:1 mixture of diastereoisomers $\delta 7.63-6.97(\mathrm{~m}, 10 \mathrm{H}), 5.61-5.35$ (m, 1H), $3.58-3.40(\mathrm{~m}, 1 \mathrm{H}), 3.25(\mathrm{~d}, J=71.6 \mathrm{~Hz}, 1 \mathrm{H}), 2.37(\mathrm{~d}, J=14.0 \mathrm{~Hz}, 1 \mathrm{H}), 2.25-2.14$ (m, 1H), $2.02-1.95(\mathrm{~m}, 1 \mathrm{H}), 1.90(\mathrm{td}, J=11.5,7.5 \mathrm{~Hz}, 2 \mathrm{H}), 1.86-1.74(\mathrm{~m}, 3 \mathrm{H}), 1.72(\mathrm{~d}, J=$ $3.5 \mathrm{~Hz}, 2 \mathrm{H}), 1.64-1.49(\mathrm{~m}, 7 \mathrm{H}), 1.39$ (ddt, $J=33.2,17.5,7.1 \mathrm{~Hz}, 9 \mathrm{H}), 1.25-1.12(\mathrm{~m}, 10 \mathrm{H})$, $1.11-0.99(\mathrm{~m}, 4 \mathrm{H}), 0.94(\mathrm{~d}, J=6.5 \mathrm{~Hz}, 3 \mathrm{H}), 0.90(\mathrm{dt}, J=6.6,2.6 \mathrm{~Hz}, 7 \mathrm{H}), 0.74-0.58(\mathrm{~m}, 1 \mathrm{H})$, $0.16(\mathrm{q}, J=12.0,10.8 \mathrm{~Hz}, 1 \mathrm{H}),-0.21(\mathrm{~d}, J=12.5 \mathrm{~Hz}, 1 \mathrm{H}),-0.57$ (s, 2H).

${ }^{13} \mathrm{C} \mathrm{NMR}\left(126 \mathrm{MHz}, \mathrm{CDCl}_{3}\right)$ 5:1 mixture of diastereoisomers $\delta 146.9,142.2,140.9,129.1,126.1$, 126.1, 125.6, 125.4, 71.1, 60.4, 59.0, 56.7, 56.4, 49.7, 46.3, 44.8, 43.1, 42.3, 41.8, 39.5, 38.5, $37.6,37.4,36.2,36.1,34.8,34.7,34.3,31.6,28.8,28.1,28.0,26.8,26.4,26.0,24.1,24.0,22.8$, 22.6, 19.1, 19.0, 15.5, 13.2, 11.1 .

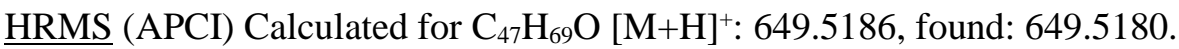




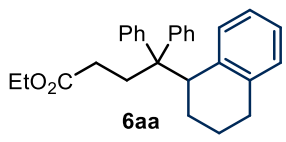

Ethyl 4,4-diphenyl-4-(1,2,3,4-tetrahydronaphthalen-1-yl)butanoate

(6aa)

Prepared according to General Procedure B using the radical precursor $\mathbf{4 b}$ (48.1 mg, $0.1 \mathrm{mmol})$, tetralin $2 \mathbf{k}(136 \mu \mathrm{L}, 1.0 \mathrm{mmol})$, and 1,1-diphenylethylene $\mathbf{5 a}(17.7 \mu \mathrm{L}, 0.1$ mmol). Multiple purifications by column chromatography resulted in poor separation from several unidentified byproducts. The yield (60\%, average of two runs) of 6aa was inferred by ${ }^{1} \mathrm{H}$ NMR analysis of the crude reaction mixture using trichloroethylene as the internal standard. An analytical amount of pure compound was isolated by preparative TLC (50:50 hexanes/toluene) to obtain a colorless oil.

${ }^{1} \mathrm{H}$ NMR $\left(500 \mathrm{MHz}, \mathrm{CDCl}_{3}\right) \delta 7.30-7.16(\mathrm{~m}, 7 \mathrm{H}), 7.16-7.10(\mathrm{~m}, 3 \mathrm{H}), 7.09-7.02(\mathrm{~m}, 4 \mathrm{H})$, $6.81(\mathrm{~d}, \mathrm{~J}=7.3 \mathrm{~Hz}, 1 \mathrm{H}), 4.06(\mathrm{qd}, \mathrm{J}=7.2,3.0 \mathrm{~Hz}, 2 \mathrm{H}), 2.67$ (ddd, $\mathrm{J}=14.0,11.6,4.1 \mathrm{~Hz}, 1 \mathrm{H})$, 2.37 (ddd, $\mathrm{J}=14.1,12.1,5.3 \mathrm{~Hz}, 1 \mathrm{H}), 2.28-2.20(\mathrm{~m}, 1 \mathrm{H}), 2.16(\mathrm{ddd}, \mathrm{J}=16.1,12.1,4.0 \mathrm{~Hz}, 1 \mathrm{H}$ ), $2.05-1.96(\mathrm{~m}, 1 \mathrm{H}), 1.88(\mathrm{ddt}, \mathrm{J}=14.3,11.8,5.9 \mathrm{~Hz}, 1 \mathrm{H}), 1.67(\mathrm{ddd}, \mathrm{J}=16.1,11.7,5.3 \mathrm{~Hz}, 1 \mathrm{H})$, $1.57-1.50(\mathrm{~m}, 1 \mathrm{H}), 1.22(\mathrm{t}, \mathrm{J}=7.1 \mathrm{~Hz}, 3 \mathrm{H}), 1.15$ (dddd, $\mathrm{J}=16.7,10.3,6.1,3.0 \mathrm{~Hz}, 1 \mathrm{H}), 0.80-$ $0.70(\mathrm{~m}, 1 \mathrm{H})$.

${ }^{13} \mathrm{C}$ NMR $\left(126 \mathrm{MHz}, \mathrm{CDCl}_{3}\right) \delta 173.8,143.5,137.0,130.8,130.3,130.2,128.5,127.8,127.8$, 127.4, 127.2, 126.3, 126.0, 125.9, 124.7, 60.2, 57.4, 44.2, 35.5, 30.7, 29.1, 25.1, 23.2, 14.2, 14.2. HRMS (ESI) Calculated for $\mathrm{C}_{28} \mathrm{H}_{30} \mathrm{NaO}_{2}[\mathrm{M}+\mathrm{Na}]^{+}:$421.2138, found: 421.2138 .

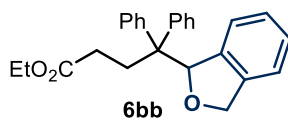

\section{Ethyl 4-(1,3-dihydroisobenzofuran-1-yl)-4,4-diphenylbutanoate (6bb)}

Prepared according to General Procedure B using the radical precursor $\mathbf{4 b}$ (48.1 mg, $0.1 \mathrm{mmol}), 1,3$-dihydrobenzofuran $2 \mathbf{2}(109 \mu \mathrm{L}, 1.0 \mathrm{mmol})$, and 1,1-diphenylethylene 5a $(17.7 \mu \mathrm{L}, 0.1 \mathrm{mmol})$. The crude mixture was purified by column chromatography $\left(\mathrm{SiO}_{2}, 60: 40\right.$ hexanes/toluene) to afford product $\mathbf{6 b b}$ as a colorless oil (21.4 $\mathrm{mg}$, $55 \%$ yield, average of two runs).

$\underline{{ }^{1} \mathrm{H} \mathrm{NMR}}\left(400 \mathrm{MHz}, \mathrm{CDCl}_{3}\right) \delta 7.39-7.33(\mathrm{~m}, 2 \mathrm{H}), 7.27-7.13(\mathrm{~m}, 9 \mathrm{H}), 7.08-6.97(\mathrm{~m}, 2 \mathrm{H})$, $6.44(\mathrm{~d}, J=7.7 \mathrm{~Hz}, 1 \mathrm{H}), 6.20(\mathrm{~d}, J=3.0 \mathrm{~Hz}, 1 \mathrm{H}), 4.88(\mathrm{~d}, J=12.1 \mathrm{~Hz}, 1 \mathrm{H}), 4.48(\mathrm{dd}, J=12.2$, $3.1 \mathrm{~Hz}, 1 \mathrm{H}), 4.07(\mathrm{q}, J=7.1 \mathrm{~Hz}, 2 \mathrm{H}), 2.78(\mathrm{ddd}, J=14.0,11.5,4.9 \mathrm{~Hz}, 1 \mathrm{H}), 2.45(\mathrm{ddd}, J=14.0$, $11.4,5.7 \mathrm{~Hz}, 1 \mathrm{H}), 2.28-2.13(\mathrm{~m}, 2 \mathrm{H}), 1.22(\mathrm{t}, J=7.1 \mathrm{~Hz}, 3 \mathrm{H})$.

${ }^{13} \mathrm{C} \mathrm{NMR}\left(101 \mathrm{MHz}, \mathrm{CDCl}_{3}\right) \delta 173.8,144.1,142.8,140.8,138.6,129.7,129.5,127.6,127.5$, 126.4, 126.3, 126.2, 123.6, 120.4, 88.9, 72.9, 60.2, 56.3, 31.6, 30.5, 14.1, 1.0.

HRMS (ESI) Calculated for $\mathrm{C}_{26} \mathrm{H}_{26} \mathrm{NaO}_{3}[\mathrm{M}+\mathrm{Na}]^{+}: 409.1774$ found: 409.1776.

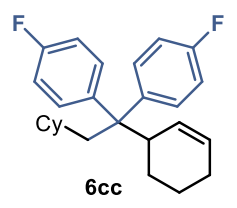

4,4'-(1-(cyclohex-2-en-1-yl)-2-cyclohexylethane-1,1-diyl)bis(fluorobenzene) (6cc)

Prepared according to General Procedure B using the radical precursor $\mathbf{1 h}$ (49.3 $\mathrm{mg}, 0.12 \mathrm{mmol})$, cyclohexene $\mathbf{2 a}(101 \mu \mathrm{L}, 1.0 \mathrm{mmol})$, and 4,4'-(ethene-1,1diyl)bis(fluorobenzene) $\mathbf{5 b}(21.6 \mathrm{mg}, 0.1 \mathrm{mmol})$. The crude mixture was purified by column chromatography $\left(\mathrm{SiO}_{2}\right.$, pentane) to afford product 6cc as a colorless oil $(25.8 \mathrm{mg}, 68 \%$ yield, average of two runs).

${ }^{1} \mathrm{H}$ NMR $\left(500 \mathrm{MHz}, \mathrm{CDCl}_{3}\right) \delta 7.17(\mathrm{pd}, J=6.6,6.2,3.1 \mathrm{~Hz}, 4 \mathrm{H}), 6.96(\mathrm{td}, J=8.8,6.3 \mathrm{~Hz}, 4 \mathrm{H})$, $5.81(\mathrm{dt}, J=10.4,2.2 \mathrm{~Hz}, 1 \mathrm{H}), 5.62(\mathrm{ddt}, J=10.3,5.1,2.6 \mathrm{~Hz}, 1 \mathrm{H}), 3.15$ (ddq, $J=9.6,4.7,2.3$ $\mathrm{Hz}, 1 \mathrm{H}), 2.03-1.82(\mathrm{~m}, 4 \mathrm{H}), 1.66$ (dddd, $J=19.4,8.8,4.4,2.2 \mathrm{~Hz}, 1 \mathrm{H}), 1.52-1.47(\mathrm{~m}, 3 \mathrm{H})$, $1.11(\mathrm{tt}, J=7.5,3.4 \mathrm{~Hz}, 1 \mathrm{H}), 1.07-0.96(\mathrm{~m}, 5 \mathrm{H}), 0.93-0.86(\mathrm{~m}, 2 \mathrm{H}), 0.85-0.74(\mathrm{~m}, 3 \mathrm{H})$. 
${ }^{13} \mathrm{C} \mathrm{NMR}\left(126 \mathrm{MHz}, \mathrm{CDCl}_{3}\right) \delta 162.0(\mathrm{~d}, J=16.2 \mathrm{~Hz}), 160.0(\mathrm{~d}, J=16.3 \mathrm{~Hz}), 141.7(\mathrm{~d}, J=3.4$ $\mathrm{Hz}), 140.1(\mathrm{~d}, J=3.3 \mathrm{~Hz}), 131.2(\mathrm{~d}, J=7.5 \mathrm{~Hz}), 131.0(\mathrm{~d}, J=7.6 \mathrm{~Hz}), \delta 129.2,128.8,114.1(\mathrm{~d}$, $J=20.8 \mathrm{~Hz}), 113.5(\mathrm{~d}, J=20.6 \mathrm{~Hz}), 54.0,46.9,41.4,35.4,35.2,33.7,26.5(\mathrm{~d}, J=8.6 \mathrm{~Hz}), 26.2$, $25.0(\mathrm{~d}, J=3.9 \mathrm{~Hz}), 22.3$.

${ }^{19} \mathrm{~F} \mathrm{NMR}\left(376 \mathrm{MHz}, \mathrm{CDCl}_{3}\right) \delta-117.7$

HRMS (APCI) Calculated for $\mathrm{C}_{26} \mathrm{H}_{31} \mathrm{~F}_{2}[\mathrm{M}+\mathrm{H}]^{+}: 381.2388$, found: 381.2380 .

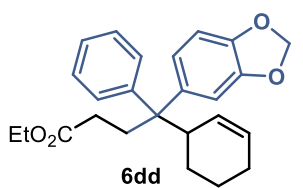

Ethyl 4-(benzo[d][1,3]dioxol-5-yl)-4-(cyclohex-2-en-1-yl)-4phenylbutanoate (6dd)

Prepared according to General Procedure B using the radical precursor $\mathbf{4 b}$ (48.1 mg, $0.1 \mathrm{mmol})$, cyclohexene $2 \mathbf{2 a}(101 \mu \mathrm{L}, 1.0 \mathrm{mmol})$, 5-(1phenylvinyl)benzo[d][1,3]dioxole 5c (22.4 mg, $0.1 \mathrm{mmol})$, and catalyst $\mathbf{C 5}$ (7.6 mg, $0.02 \mathrm{mmol})$. The crude mixture was purified by column chromatography $\left(\mathrm{SiO}_{2}, 60: 40\right.$ hexanes/toluene) to afford product 6dd as a colorless oil (29.5 $\mathrm{mg}, 73 \%$ yield, 1.1:1 dr, average of two runs).

${ }^{1} \mathrm{H} \mathrm{NMR}\left(400 \mathrm{MHz}, \mathrm{CDCl}_{3}\right)$ 1.1:1 mixture of diastereoisomers $\delta 7.32-7.19(\mathrm{~m}, 5 \mathrm{H}), 6.79-6.66$ $(\mathrm{m}, 3 \mathrm{H}), 5.98-5.94(\mathrm{~m}, 2 \mathrm{H}), 5.85-5.73(\mathrm{~m}, 1 \mathrm{H}), 5.67-5.61(\mathrm{~m}, 1 \mathrm{H}), 4.07(\mathrm{dq}, J=9.3,7.1 \mathrm{~Hz}$, 2H), $3.13(\mathrm{~d}, J=8.5 \mathrm{~Hz}, 1 \mathrm{H}), 2.50$ (ddd, $J=13.8,11.8,4.7 \mathrm{~Hz}, 1 \mathrm{H}), 2.36$ (ddd, $J=11.6,7.8,5.8$ $\mathrm{Hz}, 1 \mathrm{H}), 2.04(\mathrm{ddd}, J=16.3,11.6,4.7 \mathrm{~Hz}, 1 \mathrm{H}), 1.99-1.91(\mathrm{~m}, 1 \mathrm{H}), 1.90(\mathrm{~d}, J=5.2 \mathrm{~Hz}, 2 \mathrm{H})$, $1.80-1.68(\mathrm{~m}, 1 \mathrm{H}), 1.58-1.48(\mathrm{~m}, 1 \mathrm{H}), 1.23(\mathrm{q}, J=7.0 \mathrm{~Hz}, 3 \mathrm{H}), 1.07-0.95(\mathrm{~m}, 1 \mathrm{H}), 0.93-$ $0.84(\mathrm{~m}, 1 \mathrm{H})$.

${ }^{13} \mathrm{C} \mathrm{NMR}\left(101 \mathrm{MHz}, \mathrm{CDCl}_{3}\right)$ 1.1:1 mixture of diastereoisomers $\delta 173.9,173.8,147.3,146.7$, 145.6, 145.3, 143.0, 139.3, 137.0, 129.7, 129.3, 129.1, 129.0, 129.0, 127.8, 127.2, 126.0, 126.0, $122.7,122.4,110.6,110.0,107.3,106.8,100.9,100.8,60.3,53.6,42.0,41.7,34.3,34.1,30.2$, 30.1, 25.1, 25.0, 24.8, 24.7, 22.4, 14.2.

HRMS (ESI) Calculated for $\mathrm{C}_{25} \mathrm{H}_{28} \mathrm{NaO}_{4}[\mathrm{M}+\mathrm{Na}]^{+}: 415.1880$, found: 415.1882 .

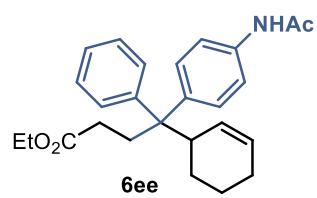

\section{Ethyl 4-(4-acetamidophenyl)-4-(cyclohex-2-en-1-yl)-4- phenylbutanoate (6ee)}

Prepared according to General Procedure B using the radical precursor $4 \mathbf{b}$ (48.1 mg, $0.1 \mathrm{mmol})$, cyclohexene 2a $(101 \mu \mathrm{L}, 1.0 \mathrm{mmol}), \mathrm{N}-(4-(1-$ phenylvinyl)phenyl)acetamide 5d (23.7 mg, $0.1 \mathrm{mmol})$, and catalyst $\mathbf{C 5}(7.6 \mathrm{mg}, 0.02 \mathrm{mmol})$. Multiple purifications by column chromatography resulted in poor separation from several unidentified byproducts. The yield (83\%,1.1:1 dr, average of two runs) of 6ee was inferred by ${ }^{1} \mathrm{H}$ NMR analysis of the crude reaction mixture using trichloroethylene as the internal standard. An analytical amount of the pure compound was isolated by preparative TLC (90:10 hexanes/EtOAc) to obtain a colorless oil.

${ }^{1} \mathrm{H} \mathrm{NMR}\left(400 \mathrm{MHz}, \mathrm{CDCl}_{3}\right)$ 1.1:1 mixture of diastereoisomers $\delta 7.43(\mathrm{~d}, J=8.6 \mathrm{~Hz}, 2 \mathrm{H}), 7.31-$ $7.27(\mathrm{~m}, 1 \mathrm{H}), 7.25-7.14(\mathrm{~m}, 6 \mathrm{H}), 5.77(\mathrm{~d}, J=10.8 \mathrm{~Hz}, 1 \mathrm{H}), 5.60(\mathrm{~d}, J=10.8 \mathrm{~Hz}, 1 \mathrm{H}), 4.06(\mathrm{qd}$, $J=7.2,2.2 \mathrm{~Hz}, 2 \mathrm{H}), 3.15(\mathrm{~s}, 1 \mathrm{H}), 2.54(\mathrm{ddd}, J=13.9,11.7,4.5 \mathrm{~Hz}, 1 \mathrm{H}), 2.45-2.33(\mathrm{~m}, 1 \mathrm{H})$, $2.20(\mathrm{~s}, 3 \mathrm{H}), 2.10-1.99(\mathrm{~m}, 1 \mathrm{H}), 1.97-1.82(\mathrm{~m}, 3 \mathrm{H}), 1.61(\mathrm{~s}, 4 \mathrm{H}), 1.22(\mathrm{td}, J=7.1,1.3 \mathrm{~Hz}, 3 \mathrm{H})$, $0.95(\mathrm{dd}, J=24.8,12.4 \mathrm{~Hz}, 1 \mathrm{H})$.

${ }^{13} \mathrm{C} \mathrm{NMR}\left(101 \mathrm{MHz}, \mathrm{CDCl}_{3}\right)$ 1.1:1 mixture of diastereoisomers $\delta 173.8,168.1,145.1,138.9$, $135.8,130.3,129.9,129.6,129.2,129.0,128.9,127.7,127.1,126.0,125.9,119.0,118.4,60.3$, 53.4, 41.6, 34.0, 30.0, 25.0, 24.6, 22.3, 14.1, 1.0.

$\underline{\text { HRMS }}$ (ESI) Calculated for $\mathrm{C}_{26} \mathrm{H}_{31} \mathrm{NaNO}_{3}[\mathrm{M}+\mathrm{Na}]^{+}:$428.2196, found: 428.2184. 
<smiles>CCOC(=O)CCC(CCCC(=O)OC)(c1ccccc1)C1C=CCCC1</smiles>

\section{4-(1-(cyclohex-2-en-1-yl)-2-cyclohexyl-1-phenylethyl)benzaldehyde} (6ff)

Prepared according to General Procedure B using the radical precursor $\mathbf{1 h}$ (49.3 mg, $0.12 \mathrm{mmol})$, cyclohexene 2a (101 $\mu \mathrm{L}, 1.0 \mathrm{mmol})$, 4-(1phenylvinyl)benzaldehyde 5e (20.8 mg, $0.1 \mathrm{mmol})$, and catalyst $\mathbf{C 5}(7.6 \mathrm{mg}, 0.02 \mathrm{mmol})$. The crude mixture was purified by column chromatography $\left(\mathrm{SiO}_{2}, 95: 5\right.$ hexanes $\left./ \mathrm{CH}_{2} \mathrm{Cl}_{2}\right)$ to afford product $\mathbf{6 f f}$ as a colorless oil (23 $\mathrm{mg}, 62 \%$ yield, $1.1: 1 \mathrm{dr}$, average of two runs).

${ }^{1} \mathrm{H} \mathrm{NMR}\left(500 \mathrm{MHz}, \mathrm{CDCl}_{3}\right)$ 1.1:1 mixture of diastereoisomers $\delta 10.02(\mathrm{~d}, J=3.3 \mathrm{~Hz}, 1 \mathrm{H}), 7.83$ $-7.77(\mathrm{~m}, 2 \mathrm{H}), 7.44(\mathrm{dd}, J=8.6,7.0 \mathrm{~Hz}, 2 \mathrm{H}), 7.31-7.18(\mathrm{~m}, 5 \mathrm{H}), 5.89$ (dq, $J=10.3,2.0 \mathrm{~Hz}$, $0.5 \mathrm{H}$ ), $5.82(\mathrm{dp}, J=10.4,1.8 \mathrm{~Hz}, 0.5 \mathrm{H}), 5.62(\mathrm{ddt}, J=10.3,4.9,2.5 \mathrm{~Hz}, 1 \mathrm{H}), 3.32-3.22(\mathrm{~m}$, 1H), 2.09 (ddd, $J=14.0,8.0,3.9 \mathrm{~Hz}, 1 \mathrm{H}), 2.03$ (ddd, $J=14.2,4.9,2.7 \mathrm{~Hz}, 1 \mathrm{H}), 1.99-1.92$ (m, $1 \mathrm{H}), 1.87(\mathrm{~d}, J=17.3 \mathrm{~Hz}, 1 \mathrm{H}), 1.56$ (qdd, $J=10.6,4.6,2.5 \mathrm{~Hz}, 3 \mathrm{H}), 1.51-1.44(\mathrm{~m}, 3 \mathrm{H}), 1.13$ (tt, $J=7.0,3.9 \mathrm{~Hz}, 1 \mathrm{H}), 1.08-0.94(\mathrm{~m}, 5 \mathrm{H}), 0.88$ (dddd, $J=11.1,7.4,5.4,1.9 \mathrm{~Hz}, 1 \mathrm{H}), 0.84-$ $0.74(\mathrm{~m}, 2 \mathrm{H})$.

${ }^{13} \mathrm{C}$ NMR $\left(101 \mathrm{MHz}, \mathrm{CDCl}_{3}\right)$ 1.1:1 mixture of diastereoisomers $\delta 192.2,192.1,154.1,152.4$, 145.3, 143.7, 134.1, 134.0, 130.5, 130.4, 129.7, 129.5, 129.3, 129.2, 128.8, 128.7, 128.2, 127.6, 127.0, 126.0, 126.0, 55.6, 46.5, 41.4, 41.2, 35.4, 35.4, 35.2, 35.2, 33.7, 33.6, 26.5, 26.5, 26.4, 26.2, 26.1, 25.0, 25.0, 22.4, 22.3.

$\underline{\text { HRMS }}$ (ESI) Calculated for $\mathrm{C}_{27} \mathrm{H}_{32} \mathrm{NaO}[\mathrm{M}+\mathrm{Na}]^{+}: 395.2345$, found: 395.2348 .

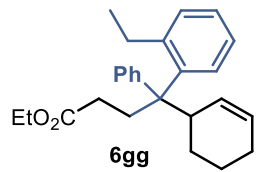

\section{Ethyl 4-(cyclohex-2-en-1-yl)-4-(2-ethylphenyl)-4-phenylbutanoate (6gg)}

Prepared according to General Procedure B using the radical precursor $\mathbf{4 b}$ (48.1 $\mathrm{mg}, \quad 0.1 \mathrm{mmol})$, cyclohexene 2a (101 $\mu \mathrm{L}, 1.0 \mathrm{mmol})$, 1-ethyl-2-(1phenylvinyl)benzene $5 \mathbf{f}(20.8 \mathrm{mg}, 0.1 \mathrm{mmol})$, and catalyst C5 (7.6 mg, 0.02 mmol). Multiple purifications by column chromatography resulted in poor separation from several unidentified byproducts. The yield (76\%, 1.5:1 d.r., average of two runs) of $\mathbf{6 g g}$ was inferred by ${ }^{1} \mathrm{H}$ NMR analysis of the crude reaction mixture using trichloroethylene as the internal standard. An analytical amount of the pure compound was isolated by preparative TLC (20:80 hexanes/toluene) to obtain a colorless oil.

${ }^{1} \mathrm{H}$ NMR $\left(400 \mathrm{MHz}, \mathrm{CDCl}_{3}\right)$ Major diastereoisomer $\delta 7.48(\mathrm{t}, J=7.0 \mathrm{~Hz}),, 7.32-7.01(\mathrm{~m}), 5.97$ $(\mathrm{d}, J=10.5 \mathrm{~Hz}, 1 \mathrm{H}), 5.77(\mathrm{dq}, J=10.3,3.4 \mathrm{~Hz}, 1 \mathrm{H}), 4.11-4.04(\mathrm{q}, 2 \mathrm{H}), 3.13(\mathrm{~m}, J=2.8 \mathrm{~Hz}$, 1H), 2.87 (ddd, $J=20.8,11.6,5.2 \mathrm{~Hz}$ ), $2.47-2.22(\mathrm{~m}), 2.09$ (ddt, $J=26.8,14.7,7.3 \mathrm{~Hz}$ ), $1.92-$ $1.74(\mathrm{~m}), 1.71-1.61(\mathrm{~m}), 1.22(\mathrm{t}, J=7.1,3 \mathrm{H}), 1.18-1.00(\mathrm{~m}), 0.87(\mathrm{t}, J=7.5 \mathrm{~Hz}, 3 \mathrm{H})$. Minor diastereoisomer $\delta 7.48(\mathrm{t}, J=7.0 \mathrm{~Hz}),, 7.32-7.01(\mathrm{~m}), 5.86(\mathrm{~s}, 1 \mathrm{H}), 5.54(\mathrm{dd}, J=10.4,3.5 \mathrm{~Hz}$, $1 \mathrm{H}), 4.11-4.04(\mathrm{q}, 2 \mathrm{H}), 3.24(\mathrm{~s}, 1 \mathrm{H}), 2.87(\mathrm{ddd}, J=20.8,11.6,5.2 \mathrm{~Hz}), 2.47-2.22(\mathrm{~m}), 2.09$ (ddt, $J=26.8,14.7,7.3 \mathrm{~Hz}), 1.92-1.74(\mathrm{~m}), 1.71-1.61(\mathrm{~m}), 1.22(\mathrm{t}, J=7.1,3 \mathrm{H}), 1.18-1.00$ (m), $0.87(\mathrm{t}, J=7.5,3 \mathrm{H})$.

${ }^{13} \mathrm{C} \mathrm{NMR}\left(101 \mathrm{MHz}, \mathrm{CDCl}_{3}\right)$ 1.5:1 mixture of diastereoisomers $\delta 173.9,144.3,141.9,141.8$, 131.6, 131.3, 130.0, 129.4, 129.0, 128.7, 128.5, 127.6, 127.3, 127.0, 126.4, 125.6, 125.6, 124.6, 124.5, 60.3, 60.2, 52.8, 52.5, 30.9, 30.5, 30.1, 30.0, 29.7, 26.7, 26.2, 25.9, 25.1, 25.0, 24.0, 22.9, 21.5, 15.3, 15.1, 14.1, 1.0.

$\underline{\text { HRMS }}$ (ESI) Calculated for $\mathrm{C}_{26} \mathrm{H}_{32} \mathrm{NaO}_{2}[\mathrm{M}+\mathrm{Na}]^{+}:$399.2295, found: 399.2281 . 


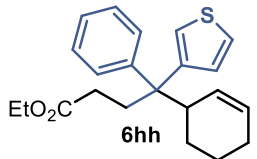

\section{Ethyl 4-(cyclohex-2-en-1-yl)-4-phenyl-4-(thiophen-3-yl)butanoate (6hh)}

Prepared according to General Procedure B using the radical precursor $4 \mathbf{b}$ (48.1 $\mathrm{mg}, \quad 0.1 \mathrm{mmol})$, cyclohexene $2 \mathbf{2 a}(101 \mu \mathrm{L}, \quad 1.0 \quad \mathrm{mmol}), \quad 3-(1-$ phenylvinyl)thiophene $\mathbf{5 g}$ (18.6 mg, $0.1 \mathrm{mmol})$, and catalyst C5 (7.6 mg, 0.02 mmol). Multiple purifications by column chromatography resulted in poor separation from several unidentified byproducts. The yield $(69 \%, 1.1: 1 \mathrm{dr}$, average of two runs) of $\mathbf{6 h h}$ was inferred by ${ }^{1} \mathrm{H}$ NMR analysis of the crude reaction mixture using trichloroethylene as the internal standard. An analytical amount of the pure compound was isolated by preparative TLC (70:30 hexanes/toluene) to obtain a colorless oil.

${ }^{1} \mathrm{H} \mathrm{NMR}\left(400 \mathrm{MHz}, \mathrm{CDCl}_{3}\right)$ 1.1:1 mixture of diastereoisomers $\delta 7.32-7.19(\mathrm{~m}, 6 \mathrm{H}), 7.13(\mathrm{dd}, J$ $=2.9,1.4 \mathrm{~Hz}, 0.5 \mathrm{H}), 6.99(\mathrm{dd}, J=3.0,1.4 \mathrm{~Hz}, 0.5 \mathrm{H}), 6.87(\mathrm{dt}, J=5.1,1.1 \mathrm{~Hz}, 1 \mathrm{H}), 5.79(\mathrm{~d}, J=$ $10.5 \mathrm{~Hz}, 0.5 \mathrm{H}), 5.72(\mathrm{~d}, J=10.4 \mathrm{~Hz}, 0.5 \mathrm{H}), 5.62(\mathrm{tq}, J=7.7,2.4 \mathrm{~Hz}, 1 \mathrm{H}), 4.12-4.04(\mathrm{~m}, 2 \mathrm{H})$, $3.16-3.00(\mathrm{~m}, 1 \mathrm{H}), 2.56$ (dddd, $J=14.3,11.8,10.0,4.6 \mathrm{~Hz}, 1 \mathrm{H}), 2.43-2.32(\mathrm{~m}, 1 \mathrm{H}), 2.19-$ $2.05(\mathrm{~m}, 1 \mathrm{H}), 2.03-1.84(\mathrm{~m}, 3 \mathrm{H}), 1.79-1.46(\mathrm{~m}, 3 \mathrm{H}), 1.23(\mathrm{td}, J=7.1,4.1 \mathrm{~Hz}, 3 \mathrm{H}), 1.11-0.96$ $(\mathrm{m}, 1 \mathrm{H})$.

${ }^{13} \mathrm{C}$ NMR $\left(101 \mathrm{MHz}, \mathrm{CDCl}_{3}\right)$ 1.1:1 mixture of diastereoisomers $\delta 173.8,146.5,144.8,144.4$, 142.5, 129.7, 129.2, 129.1, 128.9, 128.9, 128.8, 128.7, 128.5, 127.9, 127.2, 126.1, 126.1, 124.5, $123.4,122.4,122.4,60.3,52.0,51.9,43.3,42.5,34.9,33.7,30.2,30.2,29.7,25.0,25.0,24.6$, 24.6, 22.3, 22.2, 14.1 .

HRMS (ESI) Calculated for $\mathrm{C}_{22} \mathrm{H}_{26} \mathrm{NaO}_{2} \mathrm{~S}$ [M+Na] $]^{+}: 377.1546$, found: 377.1547 .

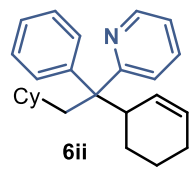

\section{2-(1-(cyclohex-2-en-1-yl)-2-cyclohexyl-1-phenylethyl)pyridine (6ii)}

Prepared according to General Procedure B using the radical precursor $\mathbf{1 h}$ (41.1 $\mathrm{mg}, 0.1 \mathrm{mmol})$, cyclohexene $\mathbf{2 a}(101 \mu \mathrm{L}, 1.0 \mathrm{mmol})$, 2-(1-phenylvinyl)pyridine $\mathbf{5 h}$ (21.7 $\mathrm{mg}, 0.12 \mathrm{mmol})$, and sodium hydrogen phosphate $(14.2 \mathrm{mg}, 0.1 \mathrm{mmol})$. The crude mixture was purified by column chromatography $\left(\mathrm{SiO}_{2}, 98: 2\right.$ hexanes/EtOAc) to afford product 6ii as a pale-yellow oil ( $21.7 \mathrm{mg}, 63 \%$ yield, $1.3: 1 \mathrm{~d} . r$. , average of two runs).

${ }^{1} \mathrm{H} \mathrm{NMR}\left(400 \mathrm{MHz}, \mathrm{CDCl}_{3}\right)$ Major diastereoisomer $\delta 8.66(\mathrm{ddd}, J=4.8,2.0,0.9 \mathrm{~Hz}, 1 \mathrm{H}), 7.50$ $(\mathrm{td}, J=7.7,1.9 \mathrm{~Hz}), 7.32-7.18(\mathrm{~m}), 7.14-7.10(\mathrm{~m}), 7.06(\mathrm{ddt}, J=15.3,8.1,1.1 \mathrm{~Hz}), 5.78(\mathrm{dt}$, $J=10.4,2.0 \mathrm{~Hz}, 1 \mathrm{H}), 5.58$ (dddt, $J=15.2,10.3,5.2,2.5 \mathrm{~Hz}), 3.65-3.55(\mathrm{~m}, 1 \mathrm{H}), 2.13-2.09$ $(\mathrm{m}, 2 \mathrm{H}), 2.00-1.81(\mathrm{~m}, 4 \mathrm{H}), 1.67-1.42(\mathrm{~m}, 12 \mathrm{H}), 1.23(\mathrm{tt}, J=11.1,3.9 \mathrm{~Hz}, 1 \mathrm{H}), 1.17-1.09$ $(\mathrm{m}, 2 \mathrm{H}), 1.01$ (tdd, $J=14.0,9.6,6.0 \mathrm{~Hz}), 0.92-0.83(\mathrm{~m}), 0.76(\mathrm{td}, J=11.8,5.6 \mathrm{~Hz})$. Minor diastereoisomer $\delta 8.63(\mathrm{ddd}, J=4.8,2.0,0.9 \mathrm{~Hz}, 1 \mathrm{H}), 7.50(\mathrm{td}, J=7.7,1.9 \mathrm{~Hz}), 7.32-7.18(\mathrm{~m})$, $7.14-7.10(\mathrm{~m}), 7.06(\mathrm{ddt}, J=15.3,8.1,1.1 \mathrm{~Hz}), 5.93(\mathrm{dp}, J=10.4,1.8 \mathrm{~Hz}, 1 \mathrm{H}), 5.58$ (dddt, $J=$ $15.2,10.3,5.2,2.5 \mathrm{~Hz}), 3.43-3.34(\mathrm{~m}, 1 \mathrm{H}), 2.20(\mathrm{~d}, J=4.4 \mathrm{~Hz}), 2.00-1.81(\mathrm{~m}), 1.67-1.42$ $(\mathrm{m}), 1.23(\mathrm{tt}, J=11.1,3.9 \mathrm{~Hz}), 1.17-1.09(\mathrm{~m}), 1.01(\mathrm{tdd}, J=14.0,9.6,6.0 \mathrm{~Hz}), 0.92-0.83(\mathrm{~m})$, $0.76(\mathrm{td}, J=11.8,5.6 \mathrm{~Hz})$.

${ }^{13} \mathrm{C} \mathrm{NMR}\left(126 \mathrm{MHz}, \mathrm{CDCl}_{3}\right)$ 1.3:1 mixture of diastereoiomers $\delta 166.1,164.7,147.9,147.4$, 145.5, 143.6, 135.0, 134.3, 130.2, 129.8, 129.5, 129.4, 128.6, 128.1, 127.3, 126.8, 125.9, 125.7, 125.5, 125.4, 120.8, 120.7, 57.1, 57.0, 45.9, 45.8, 41.7, 40.9, 35.3, 35.3, 35.1, 35.0, 33.7, 33.4, 26.7, 26.6, 26.4, 26.3, 26.2, 25.1, 25.1, 24.9, 24.7, 22.5, 22.4.

HRMS (APCI) Calculated for $\mathrm{C}_{25} \mathrm{H}_{32} \mathrm{~N}[\mathrm{M}+\mathrm{H}]^{+}: 346.2529$ found: 346.2530 . 


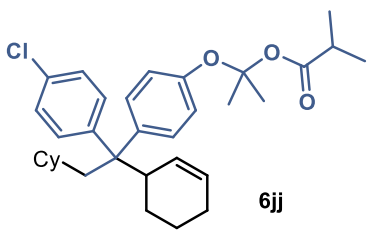

2-(4-(1-(4-Chlorophenyl)-1-(cyclohex-2-en-1-yl)-2cyclohexylethyl)phenoxy)propan-2-yl isobutyrate (6jj)

Prepared according to General Procedure B using the radical precursor 1h $(61.7 \mathrm{mg}, 0.15 \mathrm{mmol})$, cyclohexene $\mathbf{2 a}(101 \mu \mathrm{L}, 2.0 \mathrm{mmol})$, and 2(4-(1-(4-chlorophenyl)vinyl)phenoxy)propan-2-yl isobutyrate 5i (35.9 $\mathrm{mg}, 0.1 \mathrm{mmol})$. The crude mixture was purified by column chromatography $\left(\mathrm{SiO}_{2}, 80: 1\right.$ hexanes/EtOAc) to afford product $\mathbf{6 j} \mathbf{j}$ as a colorless oil $(38.2 \mathrm{mg}$, $73 \%$ yield,1.1:1 dr, average of two runs).

${ }^{1} \mathrm{H} \mathrm{NMR}\left(500 \mathrm{MHz}, \mathrm{CDCl}_{3}\right)$ 1.1:1 mixture of diasteroisomers $\delta 7.22-7.18(\mathrm{~m}, 2 \mathrm{H}), 7.16-7.11$ $(\mathrm{m}, 2 \mathrm{H}), 7.04-7.01(\mathrm{~m}, 2 \mathrm{H}), 6.76-6.69(\mathrm{~m}, 2 \mathrm{H}), 5.76(\mathrm{dd}, J=20.8,10.5 \mathrm{~Hz}, 1 \mathrm{H}), 5.58-5.52$ $(\mathrm{m}, 1 \mathrm{H}), 5.08(\mathrm{dtd}, J=12.5,6.3,2.3 \mathrm{~Hz}, 2 \mathrm{H}), 3.15-3.03(\mathrm{~m}, 1 \mathrm{H}), 1.95(\mathrm{ddd}, J=14.1,6.4,3.8$ $\mathrm{Hz}, 1 \mathrm{H}), 1.90-1.78(\mathrm{~m}, 3 \mathrm{H}), 1.59(\mathrm{~s}, 3 \mathrm{H}), 1.56(\mathrm{~s}, 3 \mathrm{H}), 1.52-1.39(\mathrm{~m}, 5 \mathrm{H}), 1.22(\mathrm{dd}, J=6.3$, $0.9 \mathrm{~Hz}, 3 \mathrm{H}), 1.20(\mathrm{dd}, J=6.2,1.9 \mathrm{~Hz}, 3 \mathrm{H}), 1.05(\mathrm{~d}, J=12.6 \mathrm{~Hz}, 2 \mathrm{H}), 1.02-0.91(\mathrm{~m}, 4 \mathrm{H}), 0.87$ $-0.80(\mathrm{~m}, 1 \mathrm{H}), 0.80-0.64(\mathrm{~m}, 2 \mathrm{H})$.

${ }^{13} \mathrm{C} \mathrm{NMR}\left(126 \mathrm{MHz}, \mathrm{CDCl}_{3}\right)$ 1.1:1 mixture of diasteroisomers $\delta 173.9,173.8,153.5,153.3,144.9$, 143.2, 139.3, 137.4, 131.4, 131.3, 131.1, 131.1, 130.3, 130.1, 129.1, 129.0, 129.0, 128.9, 128.5, 128.3, 128.0, 127.7, 127.3, 126.8, 118.5, 118.3, 118.2, 117.1, 79.0, 78.9, 68.8, 68.8, 54.1, 54.0, $46.7,46.6,41.4,41.3,35.5,35.4,35.2,35.2,33.7,33.6,33.3,26.6,26.5,26.5,26.2,26.2,25.4$, $25.3,25.3,25.2,25.1,25.0,25.0,25.0,22.4,22.3,21.6,21.5$.

$\underline{\text { HRMS }}$ (ESI) Calculated for $\mathrm{C}_{33} \mathrm{H}_{43} \mathrm{ClNaO}_{3}[\mathrm{M}+\mathrm{Na}]^{+}:$545.2793, found: 545.2787.

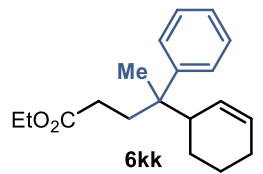

\section{Ethyl 4-(cyclohex-2-en-1-yl)-4-phenylpentanoate (6kk)}

Prepared according to General Procedure B using the radical precursor $4 \mathbf{b}$ (48.1 mg, $0.1 \mathrm{mmol})$, cyclohexene $\mathbf{2 a}(202 \mu \mathrm{L}, 2.0 \mathrm{mmol}), \alpha$-methyl styrene $\mathbf{5 j}$ (15.6 $\mu \mathrm{L}, 0.12 \mathrm{mmol}$ ), sodium hydrogen phosphate (14.2 $\mathrm{mg}, 0.1 \mathrm{mmol}$.), and catalyst $\mathbf{C 5}(7.6 \mathrm{mg}, 0.02 \mathrm{mmol})$. The crude mixture was purified by column chromatography $\left(\mathrm{SiO}_{2}, 75: 25\right.$ hexanes/toluene) to afford product $\mathbf{6 k k}$ as a colorless oil $(13.7 \mathrm{mg}, 42 \%$ yield, 1.1:1 d.r., average of two runs).

${ }^{\mathrm{fl}} \mathrm{H} \mathrm{NMR}\left(500 \mathrm{MHz}, \mathrm{CDCl}_{3}\right)$ 1.1:1 mixture of diastereoisomers $\delta 7.30(\mathrm{dt}, J=6.9,4.5 \mathrm{~Hz}, 3 \mathrm{H})$, $7.27-7.24(\mathrm{~m}, 1 \mathrm{H}), 7.20-7.16(\mathrm{~m}, 1 \mathrm{H}), 5.78(\mathrm{~s}, 1 \mathrm{H}), 5.65-5.59(\mathrm{~m}, 0.5 \mathrm{H}), 5.14(\mathrm{dp}, J=10.4$, $2.0 \mathrm{~Hz}, 0.5 \mathrm{H}$ ), 4.05 (qdd, $J=6.9,4.1,2.4 \mathrm{~Hz}, 2 \mathrm{H}), 2.52(\mathrm{dq}, J=8.4,2.7 \mathrm{~Hz}, 0.5 \mathrm{H}), 2.46$ (ddd, $J$ $=8.8,6.7,4.7 \mathrm{~Hz}, 0.5 \mathrm{H}), 2.20-2.10(\mathrm{~m}, 2 \mathrm{H}), 2.10-2.04(\mathrm{~m}, 1 \mathrm{H}), 2.03-1.95(\mathrm{~m}, 0.5 \mathrm{H}), 1.95-$ $1.77(\mathrm{~m}, 3 \mathrm{H}), 1.64$ (ddd, $J=12.3,6.0,3.0 \mathrm{~Hz}, 0.5 \mathrm{H}$ ), 1.50 (dddd, $J=19.9,12.9,7.9,2.7 \mathrm{~Hz}$, $0.5 \mathrm{H}), 1.45-1.30(\mathrm{~m}, 1.5 \mathrm{H}), 1.29-1.22(\mathrm{~m}, 2 \mathrm{H}), 1.25$ (s, 3H) $1.22-1.17$ (m, 3H), 1.07 (tdd, $J$ $=13.1,10.7,3.1 \mathrm{~Hz}, 1 \mathrm{H})$.

${ }^{13} \mathrm{C} \mathrm{NMR}\left(126 \mathrm{MHz}, \mathrm{CDCl}_{3}\right)$ 1.1:1 mixture of diastereoisomers $\delta 174.2,146.3,146.0,129.1$, $128.9,128.6,128.3,128.1,126.9,126.6,125.7,125.6,60.2$, 46.8, 46.7, 43.3, 34.6, 34.5, 29.9, 29.9, 29.7, 25.3, 25.3, 24.8, 24.3, 22.8, 22.7, 18.6, 14.2.

HRMS (ESI) Calculated for $\mathrm{C}_{19} \mathrm{H}_{26} \mathrm{NaO}_{2}[\mathrm{M}+\mathrm{Na}]^{+}: 309.1825$, found: 309.1829 .

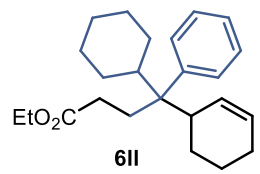

\section{Ethyl 4-(cyclohex-2-en-1-yl)-4-cyclohexyl-4-phenylbutanoate (611)}

Prepared according to General Procedure B using the radical precursor $\mathbf{4 b}$ (48.1 $\mathrm{mg}, \quad 0.1$ mmol), cyclohexene $\quad \mathbf{2 a} \quad(202 \mu \mathrm{L}, \quad 2.0 \quad \mathrm{mmol}), \quad(1-$ cyclohexylvinyl)benzene 5k $(18.6 \mathrm{mg}, 0.1 \mathrm{mmol})$, sodium hydrogen phosphate (14.2 $\mathrm{mg}, 0.1 \mathrm{mmol}$.), and catalyst $\mathbf{C 5}(7.6 \mathrm{mg}, 0.02 \mathrm{mmol})$. The crude mixture was purified by 
preparative TLC $\left(\mathrm{SiO}_{2}, 50: 50\right.$ hexanes/toluene) to afford product $61 \mathrm{l}$ as a colorless oil (16.7 $\mathrm{mg}$, $47 \%$ yield, 1.1:1 d.r., average of two runs).

${ }^{1} \mathrm{H} \mathrm{NMR}\left(500 \mathrm{MHz}, \mathrm{CDCl}_{3}\right)$ 1.1:1 mixture of diastereoisomers $\delta 7.34-7.24(\mathrm{~m}, 4 \mathrm{H}), 7.21-7.16$ $(\mathrm{m}, 1 \mathrm{H}), 5.85(\mathrm{~d}, J=10.4 \mathrm{~Hz}, 0.5 \mathrm{H}), 5.69-5.64(\mathrm{~m}, 0.5 \mathrm{H}), 5.51-5.44(\mathrm{~m}, 0.5 \mathrm{H}), 5.34(\mathrm{~d}, J=$ $10.5 \mathrm{~Hz}, 0.5 \mathrm{H}), 4.17-4.10(\mathrm{~m}, 2 \mathrm{H}), 3.22-3.13(\mathrm{~m}, 0.5 \mathrm{H}), 3.09-2.98(\mathrm{~m}, 0.5 \mathrm{H}), 2.62(\mathrm{t}, J=$ $13.9 \mathrm{~Hz}, 0.5 \mathrm{H}), 2.59-2.48(\mathrm{~m}, 1 \mathrm{H}), 2.44-2.39(\mathrm{~m}, 0.5 \mathrm{H}), 2.39-2.25(\mathrm{~m}, 1 \mathrm{H}), 2.21-2.07(\mathrm{~m}$, $1 \mathrm{H}), 2.04-1.94(\mathrm{~m}, 2 \mathrm{H}), 1.94-1.87(\mathrm{~m}, 1 \mathrm{H}), 1.86-1.79(\mathrm{~m}, 2 \mathrm{H}), 1.79-1.45(\mathrm{~m}, 4 \mathrm{H}), 1.31-$ $1.23(\mathrm{~m}, 3 \mathrm{H}), 1.04-0.91(\mathrm{~m}, 2 \mathrm{H}), 0.91-0.80(\mathrm{~m}, 2 \mathrm{H}), 0.80-0.69(\mathrm{~m}, 1 \mathrm{H}), 0.57-0.46(\mathrm{~m}$, $0.5 \mathrm{H}), 0.28(\mathrm{q}, J=12.3 \mathrm{~Hz}, 0.5 \mathrm{H})$.

${ }^{13} \mathrm{C} \mathrm{NMR}\left(126 \mathrm{MHz}, \mathrm{CDCl}_{3}\right)$ 1.1:1 mixture of diastereoisomers $\delta 174.5,174.4,143.6,132.1$, $129.8,128.8,128.0,127.5,127.4,126.3,125.5,125.4,60.3,60.2,49.2,48.1,45.6,42.2,40.3$, $38.6,30.9,30.6,30.3,29.7,28.6,28.5,28.3,28.1,28.0,27.5,27.4,27.3,26.6,26.4,25.6,25.5$, 25.4, 24.1, 23.5, 23.3, 14.3.

HRMS (ESI) Calculated for $\mathrm{C}_{24} \mathrm{H}_{34} \mathrm{NaO}_{2}[\mathrm{M}+\mathrm{Na}]^{+}: 377.2451$, found: 377.2455 .

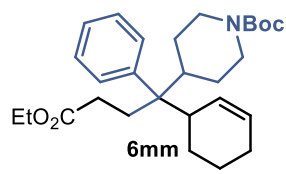

\section{Ethyl 4-(benzo[d][1,3]dioxol-5-yl)-4-(cyclohex-2-en-1-yl)-4-} phenylbutanoate $(6 \mathrm{~mm})$

Prepared according to General Procedure B using the radical precursor $\mathbf{4 b}$ (72.2 mg, $0.15 \mathrm{mmol})$, cyclohexene $2 \mathbf{a}(101 \mu \mathrm{L}, 1.0 \mathrm{mmol})$, tert-butyl 4-(1phenylvinyl)piperidine-1-carboxylate $5 \mathbf{l}$ ( $28.7 \mathrm{mg}, 0.1 \mathrm{mmol})$, sodium hydrogen phosphate (14.2 $\mathrm{mg}, 0.1 \mathrm{mmol}$.), and catalyst $\mathbf{C 5}(7.6 \mathrm{mg}, 0.02 \mathrm{mmol})$. The crude mixture was purified by column chromatography $\left(\mathrm{SiO}_{2}, 90: 10\right.$ hexanes/Et $\left.2 \mathrm{O}\right)$ to afford product $\mathbf{6 m m}$ as a colorless oil (20.0 $\mathbf{m g}$, 44\% yield, 1.1:1 d.r., average of two runs).

${ }^{1} \mathrm{H} \mathrm{NMR}\left(500 \mathrm{MHz}, \mathrm{CDCl}_{3}\right)$ 1.1:1 mixture of diastereoisomers $\delta 7.36-7.29(\mathrm{~m}, 3 \mathrm{H}), 7.27-$ $7.20(\mathrm{~m}, 2 \mathrm{H}), 5.86(\mathrm{~d}, J=10.6 \mathrm{~Hz}, 0.5 \mathrm{H}), 5.73(\mathrm{dd}, J=10.5,3.4 \mathrm{~Hz}, 0.5 \mathrm{H}), 5.52(\mathrm{dd}, J=10.4$, $3.4 \mathrm{~Hz}, 0.5 \mathrm{H}), 5.34(\mathrm{~d}, J=11.1 \mathrm{~Hz}, 0.5 \mathrm{H}), 4.20-4.01(\mathrm{~m}, 4 \mathrm{H}), 3.11(\mathrm{~d}, J=51.5 \mathrm{~Hz}, 1 \mathrm{H}), 2.68$ $-2.52(\mathrm{~m}, 4 \mathrm{H}), 2.37(\mathrm{td}, \mathrm{J}=14.8,14.1,10.2 \mathrm{~Hz}, 1 \mathrm{H}), 2.23-2.13(\mathrm{~m}, 1 \mathrm{H}), 2.08-1.90(\mathrm{~m}, 5 \mathrm{H})$, $1.75(\mathrm{dd}, J=9.9,5.6 \mathrm{~Hz}, 3 \mathrm{H}), 1.40(\mathrm{~d}, J=10.8 \mathrm{~Hz}, 9 \mathrm{H}), 1.31-1.27(\mathrm{~m}, 4 \mathrm{H}), 0.91-0.84(\mathrm{~m}$, $1 \mathrm{H})$.

${ }^{13} \mathrm{C} \mathrm{NMR}\left(101 \mathrm{MHz}, \mathrm{CDCl}_{3}\right)$ 1.1:1 mixture of diastereoisomers $\delta 174.3,174.1,154.7,154.6$, 142.6, 140.0, 131.6, 129.1, 128.7, 128.2, 127.9, 127.8, 127.7, 126.7, 125.9, 125.7, 79.2, 79.2, $60.4,60.3,48.6,47.6,44.2,41.2,40.0,38.5,31.5,30.6,30.5,28.4,28.3,28.3,27.9,25.6,25.4$, 25.4, 24.1, 23.4, 23.2, 22.6, 14.2, 14.1.

HRMS (ESI) Calculated for $\mathrm{C}_{28} \mathrm{H}_{41} \mathrm{NNaO}_{4}[\mathrm{M}+\mathrm{Na}]^{+}:$478.2928, found: 478.2927.

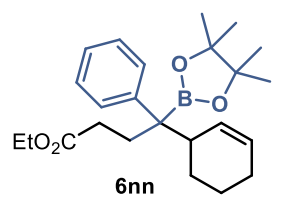

\section{Ethyl 4-(cyclohex-2-en-1-yl)-4-phenyl-4-(4,4,5,5-tetramethyl-1,3,2- dioxaborolan-2-yl)butanoate (6nn)}

Prepared according to General Procedure B using the radical precursor 4b (48.1 mg, $0.1 \mathrm{mmol})$, cyclohexene 2a $(101 \mu \mathrm{L}, 1.0 \mathrm{mmol}), 4,4,5,5-$ tetramethyl-2-(1-phenylvinyl)-1,3,2-dioxaborolane $5 \mathrm{~m}(23.0 \mathrm{mg}, 0.1 \mathrm{mmol})$, and catalyst $\mathbf{C 5}$ (7.6 $\mathrm{mg}, 0.02 \mathrm{mmol})$. The crude mixture was purified by two successive separations on column chromatography $\left(\mathrm{SiO}_{2}, 90: 10\right.$ hexanes: $\mathrm{CH}_{2} \mathrm{Cl}_{2}$ and then 50:50 hexanes/toluene) to afford product 6nn as a colorless oil (19.8 $\mathrm{mg}, 50 \%$ yield, 1.1:1 dr, average of two runs).

$\underline{{ }^{1} \mathrm{H} \mathrm{NMR}}\left(500 \mathrm{MHz}, \mathrm{CDCl}_{3}\right)$ 1.1:1 mixture of diastereoisomers $\delta 7.37-7.33(\mathrm{~m}, 4 \mathrm{H}), 7.31-7.27$ (m, 4H), $7.20-7.15(\mathrm{~m}, 2 \mathrm{H}), 5.81(\mathrm{dp}, J=10.3,1.7 \mathrm{~Hz}, 1 \mathrm{H}), 5.74-5.68(\mathrm{~m}, 1 \mathrm{H}), 5.66-5.59$ 
$(\mathrm{m}, 2 \mathrm{H}), 4.08(\mathrm{qd}, J=7.1,1.1 \mathrm{~Hz}, 4 \mathrm{H}), 2.82-2.74(\mathrm{~m}, 1 \mathrm{H}), 2.70-2.62(\mathrm{~m}, 1 \mathrm{H}), 2.45(\mathrm{ddd}, J=$ 14.6, 12.5, 4.7 Hz, 1H), $2.40-2.27(\mathrm{~m}, 2 \mathrm{H}), 2.25-2.17(\mathrm{~m}, 1 \mathrm{H}), 2.17-2.08(\mathrm{~m}, 2 \mathrm{H}), 2.06-$ $1.98(\mathrm{~m}, 2 \mathrm{H}), 1.97-1.88(\mathrm{~m}, 4 \mathrm{H}), 1.77(\mathrm{dd}, J=4.6,2.5 \mathrm{~Hz}, 1 \mathrm{H}), 1.74-1.65(\mathrm{~m}, 2 \mathrm{H}), 1.58-1.46$ $(\mathrm{m}, 1 \mathrm{H}), 1.45-1.38(\mathrm{~m}, 2 \mathrm{H}), 1.30(\mathrm{~s}, 12 \mathrm{H}), 1.27(\mathrm{~d}, J=3.7 \mathrm{~Hz}, 12 \mathrm{H}), 1.23(\mathrm{td}, J=7.1,2.1 \mathrm{~Hz}$, $6 \mathrm{H}), 0.93-0.82(\mathrm{~m}, 2 \mathrm{H})$.

${ }^{13} \mathrm{C}$ NMR $\left(101 \mathrm{MHz}, \mathrm{CDCl}_{3}\right)$ 1.1:1 mixture of diastereoisomers $\delta 174.4,143.8,143.1,130.7$, 129.5, 128.8, 128.7, 128.6, 128.0, 127.9, 127.3, 125.4, 125.4, 83.5, 83.4, 60.1, 60.0, 43.9, 42.5, 31.5, 31.1, 30.6, 29.1, 26.0, 25.4, 25.3, 25.3, 25.0, 25.0, 24.8, 24.8, 24.6, 23.0, 22.8, 14.2.

HRMS (ESI) Calculated for $\mathrm{C}_{24} \mathrm{H}_{35} \mathrm{NaBO}_{4}[\mathrm{M}+\mathrm{Na}]^{+}: 420.2557$, found: 420.2551 .

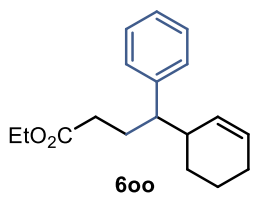

\section{Ethyl 4-(cyclohex-2-en-1-yl)-4-phenylbutanoate (6oo)}

Prepared according to General Procedure B using the radical precursor $\mathbf{4 b}$ (48.1 mg, $0.1 \mathrm{mmol})$, cyclohexene $\mathbf{2 a}(202 \mu \mathrm{L}, 2.0 \mathrm{mmol})$, styrene $\mathbf{5 n}(14 \mu \mathrm{L}, 0.12$ mmol), sodium hydrogen phosphate (14.2 mg, $0.1 \mathrm{mmol}$ ), and catalyst $\mathbf{C 5}$ (7.6 $\mathrm{mg}, 0.02 \mathrm{mmol})$. The crude mixture was purified by column chromatography $\left(\mathrm{SiO}_{2}, 75: 25\right.$ hexanes/toluene) to afford product $\mathbf{6 0 0}$ as a colorless oil (12.6 $\mathrm{mg}, 42 \%$ yield, 1.1:1 d.r., average of two runs).

$\left.\underline{{ }^{1} \mathrm{H} \mathrm{NMR}}\left(500 \mathrm{MHz}, \mathrm{CDCl}_{3}\right)\right)$ 1.1:1 mixture of diastereoisomers $\delta 7.28(\mathrm{t}, J=7.6 \mathrm{~Hz}, 2 \mathrm{H}), 7.22$ $-7.17(\mathrm{~m}, 1 \mathrm{H}), 7.13(\mathrm{td}, J=8.0,7.6,1.3 \mathrm{~Hz}, 2 \mathrm{H}), 5.89(\mathrm{dd}, J=10.3,2.5 \mathrm{~Hz}, 0.5 \mathrm{H}), 5.78-5.71$ $(\mathrm{m}, 0.5 \mathrm{H}), 5.66-5.58(\mathrm{~m}, 0.5 \mathrm{H}), 5.35(\mathrm{~d}, J=10.2 \mathrm{~Hz}, 0.5 \mathrm{H}), 4.09-4.03(\mathrm{~m}, 2 \mathrm{H}), 2.45-2.33$ (m, 1H), $2.27(\mathrm{dtd}, J=16.8,8.1,3.5 \mathrm{~Hz}, 1 \mathrm{H}), 2.21-2.13(\mathrm{~m}, 0.5 \mathrm{H}), 2.09-2.04(\mathrm{~m}, 2 \mathrm{H}), 1.97-$ $1.83(\mathrm{~m}, 3 \mathrm{H}), 1.77-1.68(\mathrm{~m}, 0.5 \mathrm{H}), 1.62(\mathrm{dq}, J=11.2,4.3,3.7 \mathrm{~Hz}, 0.5 \mathrm{H}), 1.53-1.37(\mathrm{~m}, 1 \mathrm{H})$, $1.36-1.27(\mathrm{~m}, 1 \mathrm{H}), 1.20(\mathrm{td}, J=7.0,1.8 \mathrm{~Hz}, 3 \mathrm{H}), 1.14-1.06(\mathrm{~m}, 0.5 \mathrm{H})$.

${ }^{13} \mathrm{C} \mathrm{NMR}\left(126 \mathrm{MHz}, \mathrm{CDCl}_{3}\right) 1.1: 1$ mixture of diastereoisomers $\delta 173.8,173.7,143.2,143.1$, 130.2, 129.6, 128.5, 128.4, 128.3, 128.3, 128.3, 127.9, 127.5, 126.2, 60.1, 50.8, 50.7, 41.1, 40.6, $32.8,32.6,28.0,27.6,27.3,27.0,25.4,25.3,21.9,21.1,14.2$.

HRMS (ESI) Calculated for $\mathrm{C}_{18} \mathrm{H}_{24} \mathrm{NaO}_{2}[\mathrm{M}+\mathrm{Na}]^{+}: 295.1669$, found: 295.1672 .

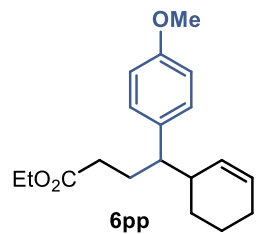

\section{Ethyl 4-(cyclohex-2-en-1-yl)-4-(4-methoxyphenyl)butanoate (6pp)}

Prepared according to General Procedure B using the radical precursor $\mathbf{4 b}$ (48.1 $\mathrm{mg}, \quad 0.1 \mathrm{mmol})$, cyclohexene $2 \mathrm{a}(202 \mu \mathrm{L}, 2.0 \mathrm{mmol})$, 1-methoxy-4vinylbenzene $50(16.2 \mu \mathrm{L}, 0.12 \mathrm{mmol})$, sodium hydrogen phosphate $(14.2 \mathrm{mg}$, $0.1 \mathrm{mmol}$.), and catalyst $\mathbf{C 5}(7.6 \mathrm{mg}, 0.02 \mathrm{mmol})$. The crude mixture was purified by column chromatography $\left(\mathrm{SiO}_{2}, 33: 66\right.$ hexanes/toluene) to afford product $\mathbf{6 p p}$ as a colorless oil (11.8 mg, $40 \%$ yield, 1.1:1 d.r., average of two runs).

${ }^{1} \mathrm{H} \mathrm{NMR}\left(500 \mathrm{MHz}, \mathrm{CDCl}_{3}\right)$ 1.1:1 mixture of diastereoisomers $\delta 7.06-7.01(\mathrm{~m}, 2 \mathrm{H}), 6.85-6.80$ $(\mathrm{m}, 2 \mathrm{H}), 5.86(\mathrm{dd}, J=10.2,2.5 \mathrm{~Hz}, 0.5 \mathrm{H}), 5.77-5.69(\mathrm{~m}, 0.5 \mathrm{H}), 5.62(\mathrm{dq}, J=10.0,3.3,2.9 \mathrm{~Hz}$, $0.5 \mathrm{H}), 5.36(\mathrm{ddd}, J=10.3,2.3,1.2 \mathrm{~Hz}, 0.5 \mathrm{H}), 4.06(\mathrm{qd}, J=7.2,1.5 \mathrm{~Hz}, 2 \mathrm{H}), 3.79(\mathrm{~s}, 6 \mathrm{H}), 2.39-$ $2.29(\mathrm{~m}, 1 \mathrm{H}), 2.24$ (dddd, $J=13.1,8.9,7.2,3.7 \mathrm{~Hz}, 1 \mathrm{H}), 2.17-2.09(\mathrm{~m}, 0.5 \mathrm{H}), 2.09-2.03(\mathrm{~m}$, 2H), $1.93(\mathrm{dp}, J=7.4,1.7 \mathrm{~Hz}, 2 \mathrm{H}), 1.88-1.78(\mathrm{~m}, 1 \mathrm{H}), 1.71(\mathrm{dq}, J=9.0,4.6 \mathrm{~Hz}, 0.5 \mathrm{H}), 1.67-$ $1.55(\mathrm{~m}, 2 \mathrm{H}), 1.54-1.45(\mathrm{~m}, 0.5 \mathrm{H}), 1.41(\mathrm{tdd}, J=11.7,6.1,2.9 \mathrm{~Hz}, 1 \mathrm{H}), 1.34-1.24(\mathrm{~m}, 3 \mathrm{H})$, $1.20(\mathrm{td}, J=7.1,1.7 \mathrm{~Hz}, 3 \mathrm{H}), 1.10$ (dddd, $J=13.1,10.4,7.6,2.5 \mathrm{~Hz}, 0.5 \mathrm{H}), 0.91-0.79(\mathrm{~m}, 0.5 \mathrm{H})$. ${ }^{13} \mathrm{C} \mathrm{NMR}\left(126 \mathrm{MHz}, \mathrm{CDCl}_{3}\right)$ 1.1:1 mixture of diastereoisomers $\delta 173.9,173.8,158.0,158.0$, 135.2, 135.1, 130.2, 129.8, 129.4, 129.3, 129.1, 128.3, 127.8, 113.8, 113.6, 113.6, 60.1, 55.2, $49.9,49.8,41.2,40.7,32.8,32.6,29.7,28.2,27.7,27.2,27.1,25.4,25.3,21.9,21.1,14.2$. 
$\underline{\text { HRMS }}$ (ESI) Calculated for $\mathrm{C}_{19} \mathrm{H}_{29} \mathrm{NaO}_{3}[\mathrm{M}+\mathrm{Na}]^{+}: 325.1774$, found: 325.1778 .

\section{D.3.3 Telescoped Three-component C-H Allylic benzylation}

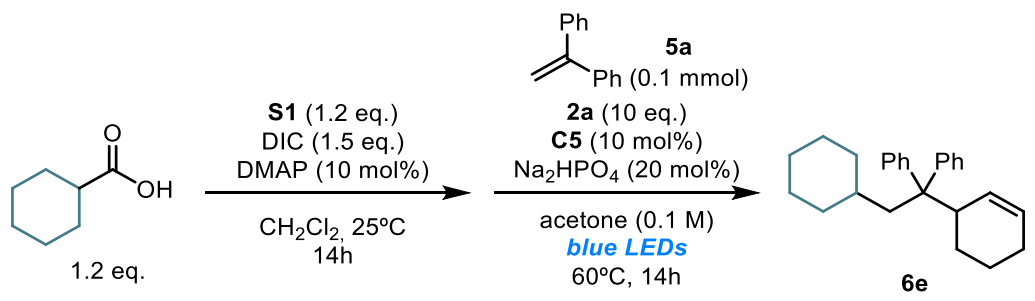

An oven-dried glass vial, was charged with cyclohexanecarboxylic acid (15.4 mg, 1.2 equiv.), S1 (36.1 mg, 1.2 equiv.), and DMAP (1.2 mg, 0.1 equiv.). The vial was purged with argon before adding $1 \mathrm{~mL}$ of degassed acetone, followed by dropwise addition of DIC ( $23.5 \mu \mathrm{L}, 1.5$ equiv.). The vial was sealed with parafilm and the reaction stirred at $25^{\circ} \mathrm{C}$ for $14 \mathrm{~h} . \mathrm{CH}_{2} \mathrm{Cl}_{2}$ was evaporated under reduced pressure. $\mathbf{C 5}$ (3.8 mg, $0.01 \mathrm{mmol}, 0.1$ equiv.) and $\mathrm{Na}_{2} \mathrm{HPO}_{4}(2.8 \mathrm{mg}, 0.02 \mathrm{mmol}$, 0.2 equiv.) were sequentially added and the vial purged with argon. 1,1-diphenylethylene $\mathbf{5 a}$ (17.7 $\mu \mathrm{L}, 0.1 \mathrm{mmol})$ and cyclohexene $\mathbf{2 a}(101 \mu \mathrm{L}, 1 \mathrm{mmol}, 10$ equiv.) were finally added, followed by $800 \mu \mathrm{L}$ of argon-sparged HPLC grade acetone. The vial was sealed with Parafilm, and then placed in the irradiation setup, maintained at a temperature of $60^{\circ} \mathrm{C}$. The reaction was stirred for $14 \mathrm{~h}$, then the solvent was evaporated and the crude mixture purified by column chromatography $\left(\mathrm{SiO}_{2}\right.$, pentane) to afford product $\mathbf{6 e}$ as a colorless oil (19.3 $\mathrm{mg}, 56 \%$ yield).
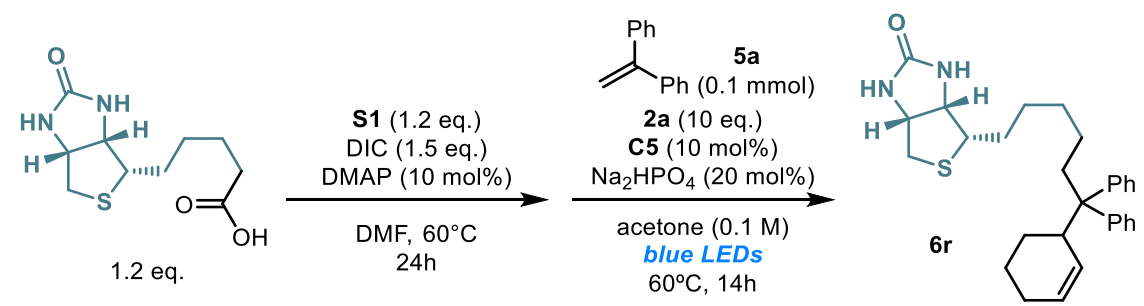

An oven-dried glass vial, was charged with D-biotin (29.3 mg, 1.2 equiv.), S1 (36.1 mg, 1.2 equiv.), and DMAP (1.2 mg, 0.1 equiv.). The vial was purged with argon before adding $1 \mathrm{~mL}$ degassed DMF, followed by dropwise addition of DIC ( $23.5 \mu \mathrm{L}, 1.5$ equiv.). The vial was sealed with parafilm and the reaction stirred at $60^{\circ} \mathrm{C}$ for $24 \mathrm{~h}$. DMF was then removed at $60^{\circ} \mathrm{C}$ under high vacuum. C5 (3.8 mg, $0.01 \mathrm{mmol}, 0.1$ equiv.), and $\mathrm{Na}_{2} \mathrm{HPO}_{4}$ ( $2.8 \mathrm{mg}, 0.02 \mathrm{mmol}, 0.2$ equiv.), were added and the vial purged with argon again. 1,1-diphenylethylene $\mathbf{5 a}(17.7 \mu \mathrm{L}, 0.1 \mathrm{mmol})$ and cyclohexene $2 \mathbf{a}(101 \mu \mathrm{L}, 1 \mathrm{mmol}, 10$ equiv.) were finally added, followed by $800 \mu \mathrm{L}$ of argonsparged HPLC grade acetone. The vial was sealed with Parafilm, and then placed in the irradiation setup, maintained at a temperature of $60^{\circ} \mathrm{C}$. The reaction was stirred for $14 \mathrm{~h}$, then the solvent was evaporated and the crude mixture purified by flash column chromatography. Multiple purifications by column chromatography resulted in poor separation from several unidentified byproducts. The yield (37\%, average of two runs, 1.1:1 d.r.) of $6 \mathbf{r}$ was inferred by ${ }^{1} \mathrm{H}$ NMR analysis of the crude reaction mixture using trichloroethylene as the internal standard. An analytical amount of the pure compound was isolated by preparative TLC (70:30 hexanes/toluene) to obtain a colorless oil. 


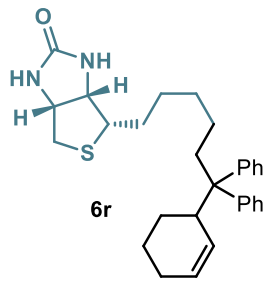

(3aS,4S,6aR)-4-(6-(cyclohex-2-en-1-yl)-6,6-diphenylhexyl)tetrahydro1H-thieno[3,4-d]imidazol-2(3H)-one (6r)

${ }^{1} \mathrm{H}$ NMR $(400 \mathrm{MHz}, \mathrm{MeOD}) \delta 7.36-7.29(\mathrm{~m}, 6 \mathrm{H}), 7.26(\mathrm{dd}, J=7.9,4.1 \mathrm{~Hz}$, $4 \mathrm{H}), 5.88(\mathrm{~d}, J=10.6 \mathrm{~Hz}, 1 \mathrm{H}), 5.67-5.56(\mathrm{~m}, 1 \mathrm{H}), 4.53(\mathrm{ddd}, J=7.9,5.0$, $1.0 \mathrm{~Hz}, 1 \mathrm{H}), 4.30(\mathrm{dd}, J=7.9,4.5 \mathrm{~Hz}, 1 \mathrm{H}), 3.30(\mathrm{~s}, 1 \mathrm{H}), 3.18(\mathrm{dt}, J=8.7,5.3$

$\mathrm{Hz}, 1 \mathrm{H}), 3.01-2.94(\mathrm{~m}, 1 \mathrm{H}), 2.76(\mathrm{~d}, J=12.7 \mathrm{~Hz}, 1 \mathrm{H}), 2.19(\mathrm{dd}, J=10.7$, $5.9 \mathrm{~Hz}, 1 \mathrm{H}), 1.94(\mathrm{t}, J=18.0 \mathrm{~Hz}, 2 \mathrm{H}), 1.74-1.59(\mathrm{~m}, 4 \mathrm{H}), 1.57-1.48(\mathrm{~m}, 1 \mathrm{H}), 1.35(\mathrm{~d}, J=15.3$ $\mathrm{Hz}, 6 \mathrm{H}), 1.15-0.95(\mathrm{~m}, 4 \mathrm{H})$.

${ }^{13} \mathrm{C}$ NMR $(101 \mathrm{MHz}, \mathrm{MeOD}) \delta 163.2,144.7,142.6,128.1,127.8,127.7,126.3,125.5,125.0$, 123.9, 123.8, 60.4, 58.7, 54.2, 52.6, 39.4, 39.3, 38.1, 37.5, 28.2, 27.8, 27.2, 26.7, 26.6, 23.2, 23.0, 22.4, 20.6.

$\underline{\text { HRMS }}$ (ESI) Calculated for $\mathrm{C}_{29} \mathrm{H}_{36} \mathrm{~N}_{2} \mathrm{NaOS}[\mathrm{M}+\mathrm{Na}]^{+}: 483.2441$, found: 483.2433 .

E. Mechanistic Studies

E.1 UV-Vis Measurements

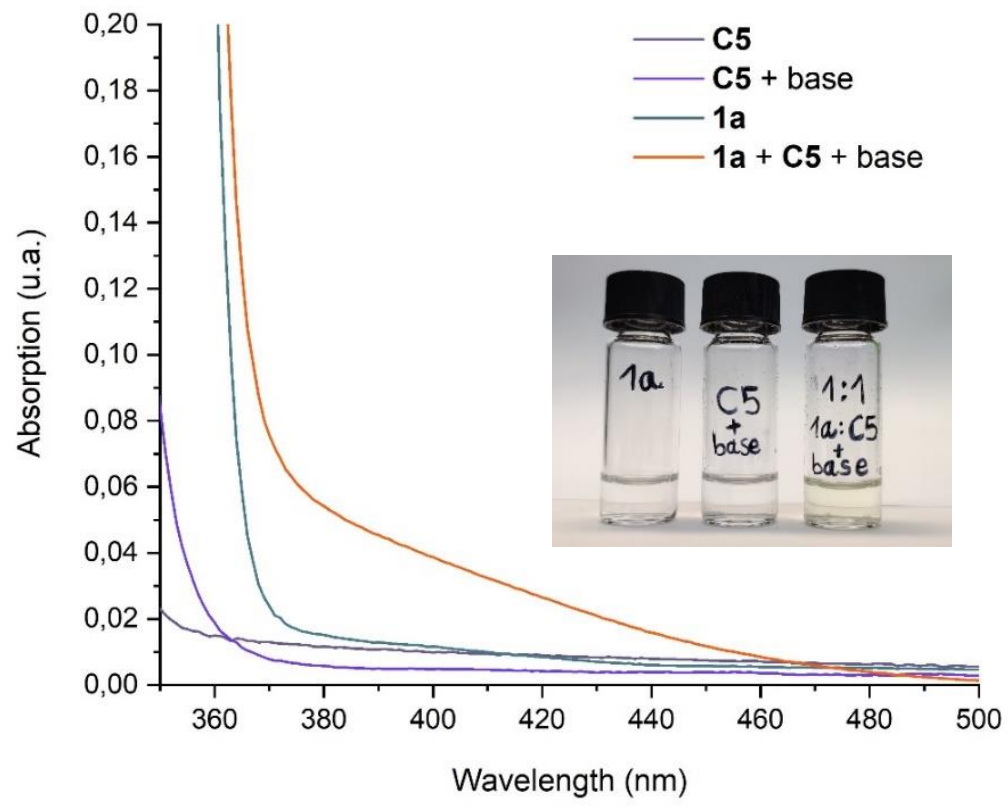

Figure S8. Optical absorption spectra, recorded in acetone in $1 \mathrm{~mm}$ path quartz cuvettes using a Shimadzu 2401PC UV-vis spectrophotometer, and visual appearance of the separate reaction components and of the colored EDA complex between catalyst $\mathbf{C 5}$ and 1a. $[\mathbf{1 a}]=0.10 \mathrm{M},[\mathbf{C 5}]=0.02 \mathrm{M},\left[\mathrm{Na}_{2} \mathrm{HPO}_{4}\right]=0.02 \mathrm{M}$. 


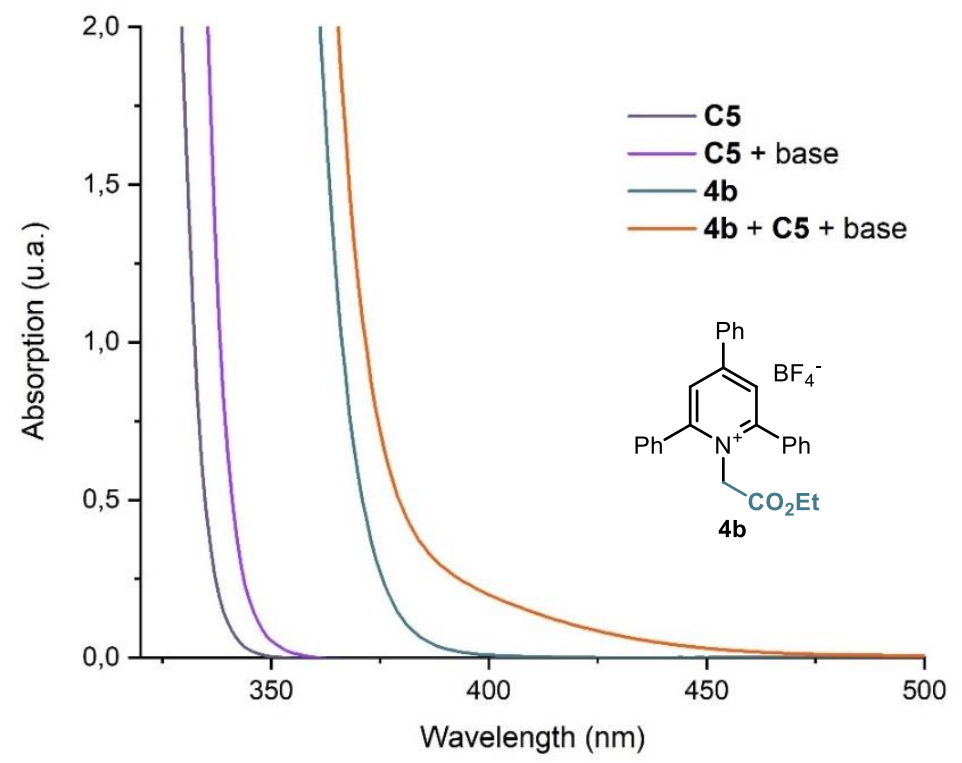

Figure S9. Optical absorption spectra, recorded in acetone in $1 \mathrm{~mm}$ path quartz cuvettes using a Shimadzu 2401PC UV-vis spectrophotometer of the colored EDA complex between catalyst $\mathbf{C 5}$ and $\mathbf{4 b}$. $[\mathbf{4 b}]=0.10 \mathrm{M},[\mathbf{C 5}]=0.01 \mathrm{M}$, $\left[\mathrm{Na}_{2} \mathrm{HPO}_{4}\right]=0.02 \mathrm{M}$.

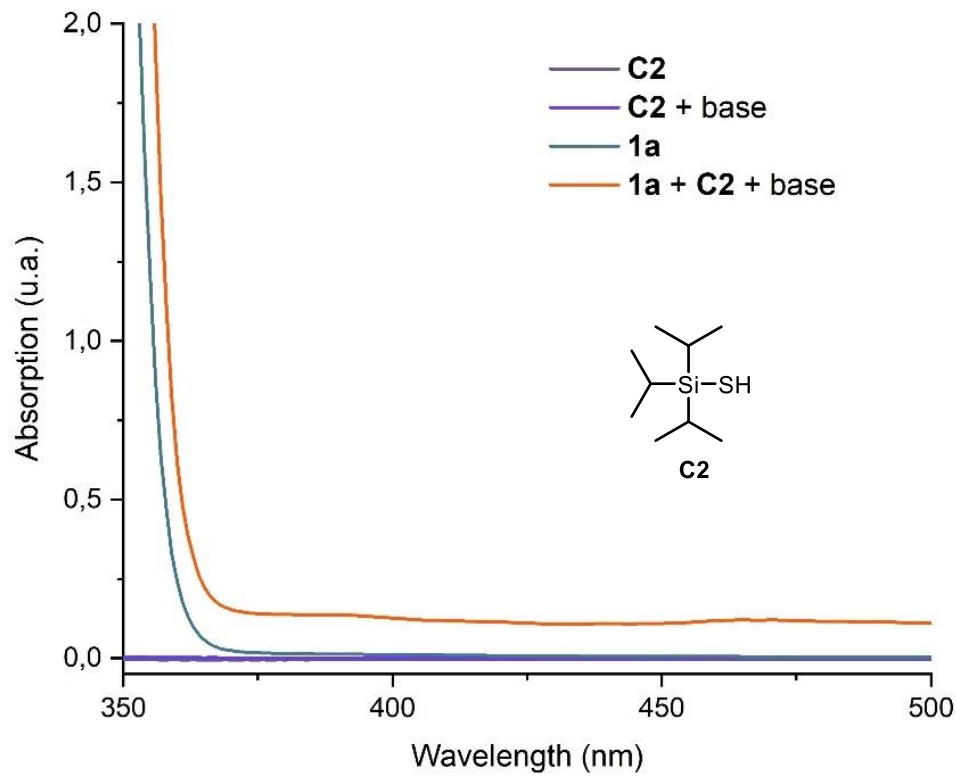

Figure S10. Optical absorption spectra, recorded in acetone in $1 \mathrm{~mm}$ path quartz cuvettes using a Shimadzu 2401PC UV-vis spectrophotometer of the colored EDA complex between catalyst $\mathbf{C 2}$ and $\mathbf{1 a}$. $[\mathbf{1 a}]=0.10 \mathrm{M},[\mathbf{C 2}]=0.02 \mathrm{M}$, $\left[\mathrm{Na}_{2} \mathrm{HPO}_{4}\right]=0.02 \mathrm{M}$. 


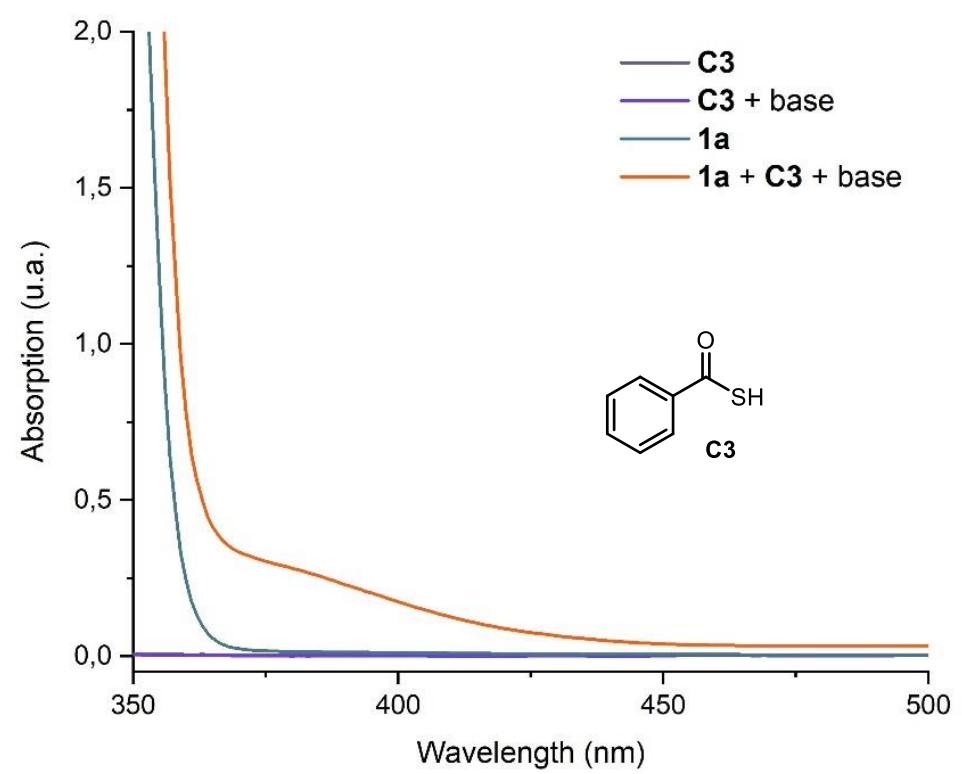

Figure S11. Optical absorption spectra, recorded in acetone in $1 \mathrm{~mm}$ path quartz cuvettes using a Shimadzu 2401PC UV-vis spectrophotometer of the colored EDA complex between catalyst $\mathbf{C 3}$ and 1a. $[\mathbf{1 a}]=0.10 \mathrm{M},[\mathbf{C 3}]=0.02 \mathrm{M}$, $\left[\mathrm{Na}_{2} \mathrm{HPO}_{4}\right]=0.02 \mathrm{M}$.

\section{E.2 Cyclic Voltammetry Measurements}

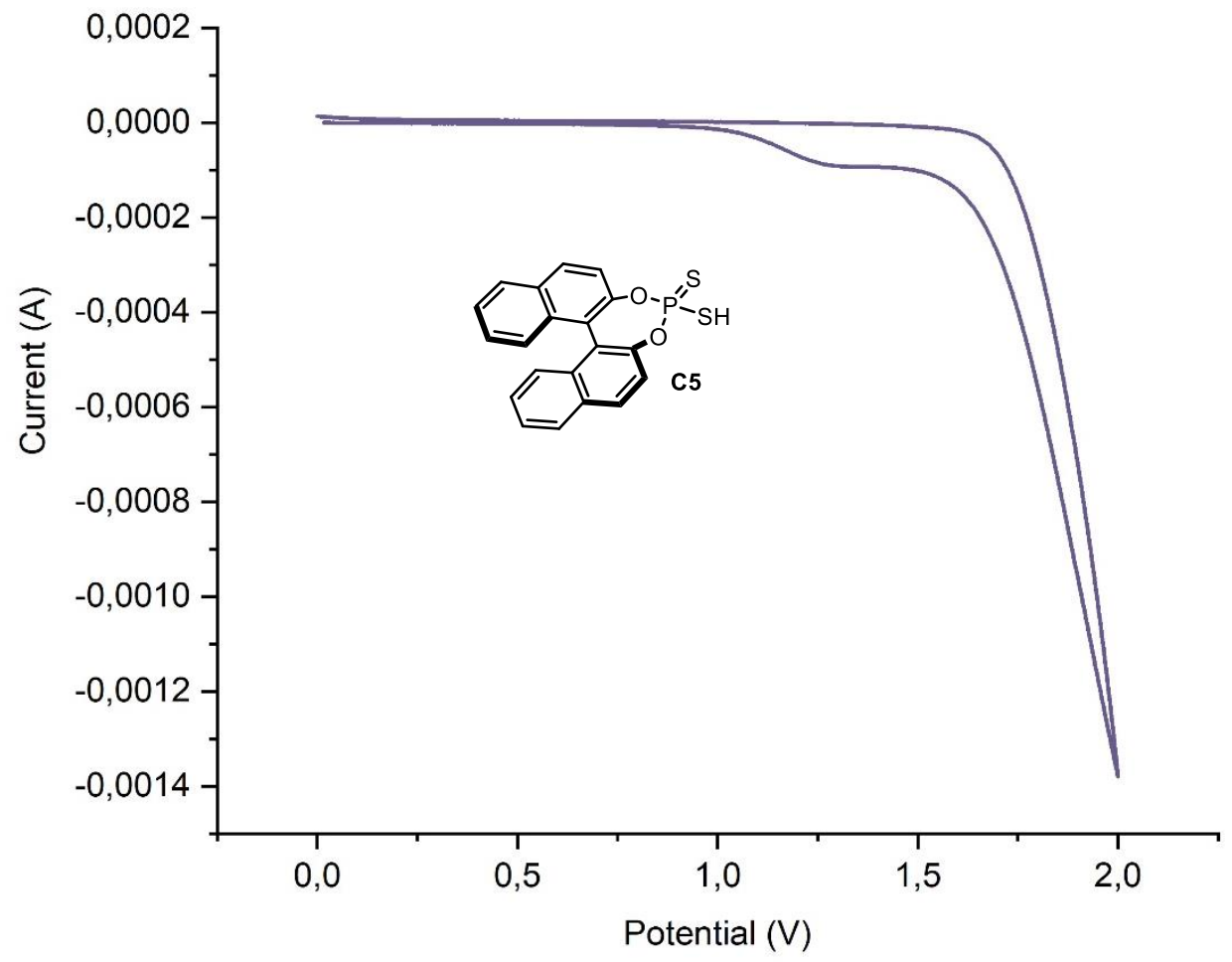

Figure S12. Cyclic voltammogram for catalyst $\mathbf{C 5}[0.02 \mathrm{M}]$ in $[0.1 \mathrm{M}] \mathrm{TBAPF} 6$ in $\mathrm{CH}_{3} \mathrm{CN}$. Measurement started by oxidation from 0 to $+2.0 \mathrm{~V}$ and finishing at $0 \mathrm{~V}$. Platinum disk working electrode, $\mathrm{Ag} / \mathrm{AgCl}(\mathrm{NaCl} 3 \mathrm{M})$ reference electrode, Pt wire auxiliary electrode. Sweep rate: $500 \mathrm{mV} / \mathrm{s}$. One irreversible oxidation observed at $+1.28 \mathrm{~V}$. 


\section{E.3 Regioselectivity Study}

Non-symmetrical cyclohexene derivatives $\mathbf{2 d}, \mathbf{2} \mathbf{h}$ and $\mathbf{2 i}$ were submitted to the reaction conditions following the General Procedure B, using thiol catalysts with different steric hindrance: catalysts C5, C6, and $\mathbf{C 7}$ were tested. The yields of products and ratio of regioisomers for each combination are reported in Table $\mathrm{S} 2$.

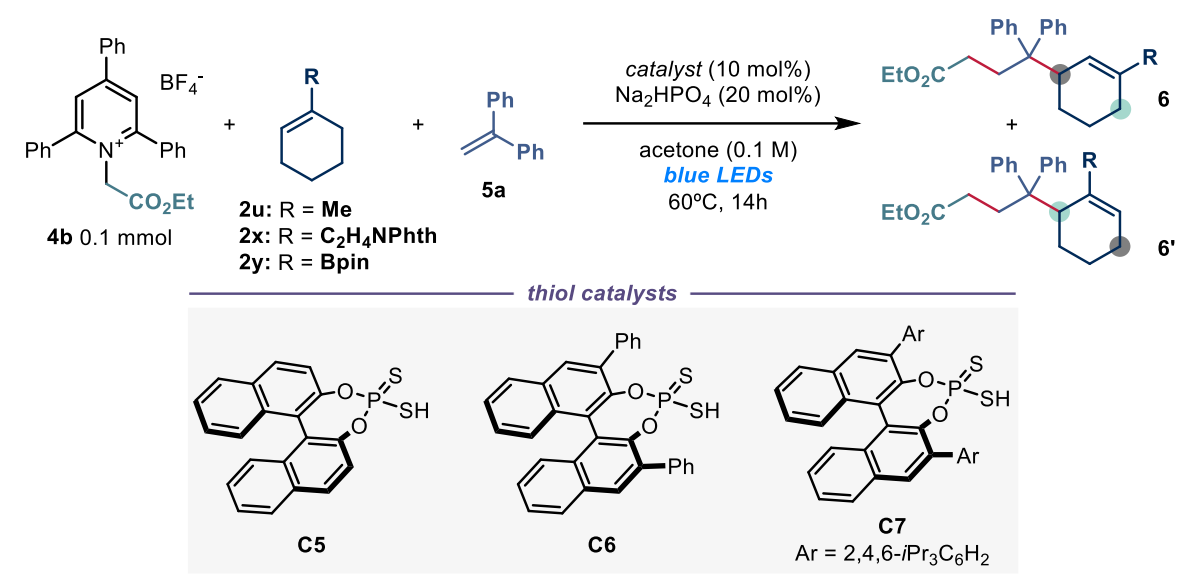

\begin{tabular}{|c|c|c|c|c|}
\hline entry & $\mathrm{R}$ & catalyst & yield $6+\mathbf{6}^{\prime}(\%)$ & $6: 6^{, b}$ \\
\hline 1 & \multirow{3}{*}{$\operatorname{Me}(\mathbf{2 d})$} & C5 & 67 & 5:1 (6u:6u') \\
\hline 2 & & C6 & 37 & $5: 1\left(6 u: 6 u^{\prime}\right)$ \\
\hline 3 & & $\mathrm{C7}$ & $26^{a}$ & 4:1 (6u:6u') \\
\hline 4 & \multirow{3}{*}{$\mathrm{C}_{2} \mathrm{H}_{4} \mathrm{NPhth}(\mathbf{2 h})$} & C5 & 62 & $6: 1\left(6 x: 6 x^{3}\right)$ \\
\hline 5 & & C6 & 54 & $7: 1\left(6 x: 6 x^{\prime}\right)$ \\
\hline 6 & & C7 & 48 & $10: 1$ (6x:6x') \\
\hline 7 & \multirow{3}{*}{ Bpin (2i) } & C5 & 45 & $1: 1.2(\mathbf{6 y} \mathbf{y}: 6 \mathbf{y})$ \\
\hline 8 & & C6 & $50^{\mathrm{a}}$ & 4:1 (6y':6y) \\
\hline 9 & & $\mathrm{C7}$ & $19^{a}$ & $4: 1\left(6 y^{\prime}: 6 y\right)$ \\
\hline
\end{tabular}

Table S2. Regioselectivity studies using non-symmetrical cyclohexene derivatives. a ${ }^{\text {Yield determined by }{ }^{1} \mathrm{H} \text { NMR }}$ analysis. ${ }^{b}$ Regioisomers ratio determined by ${ }^{1} \mathrm{H}$ NMR analysis. 

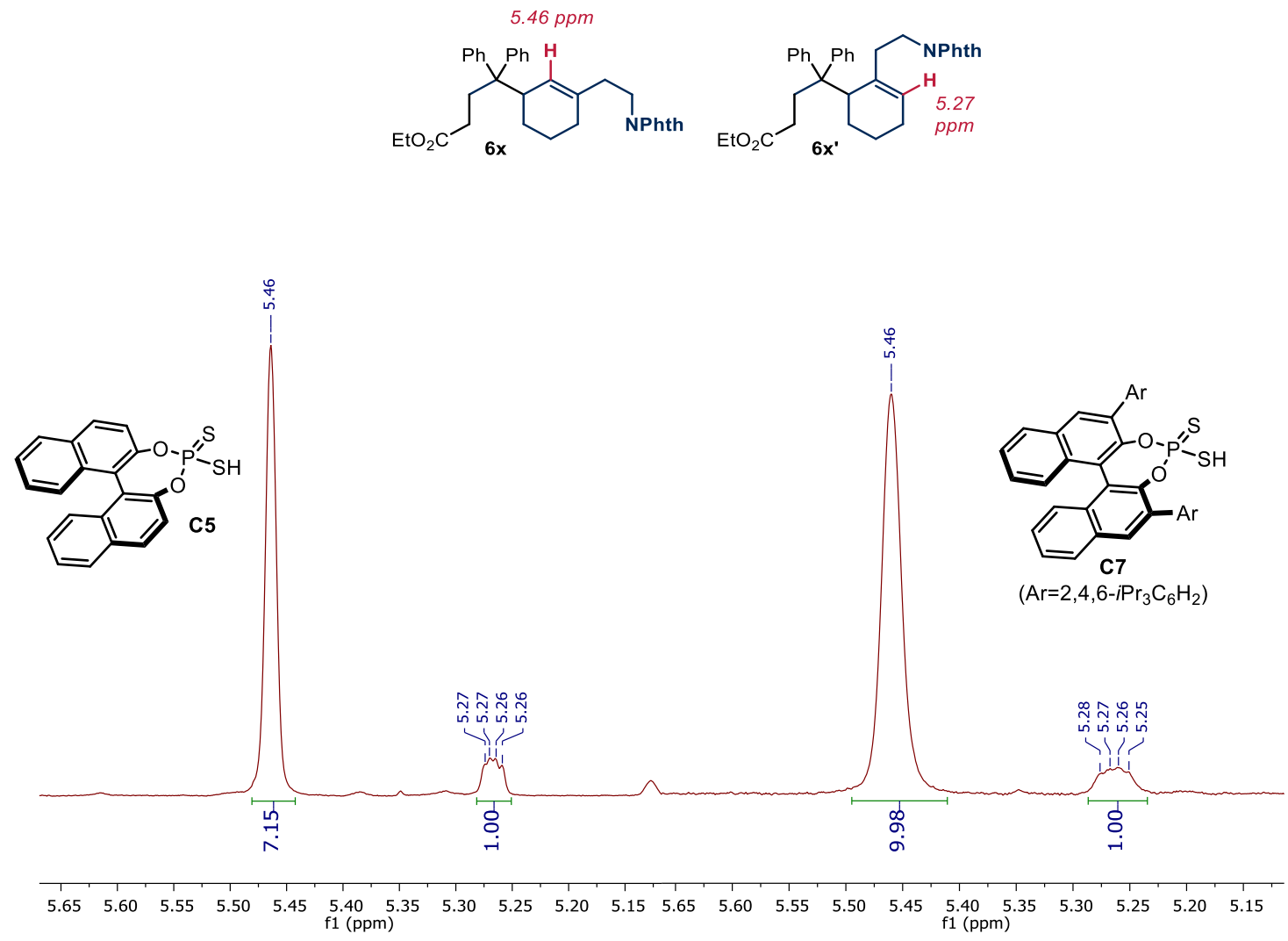

Figure S13. Regioselectivity study of $6 \mathbf{x}$ and $6 \mathbf{x}^{\prime}$ using catalysts $\mathbf{C 5}$ and $\mathbf{C 7}$.

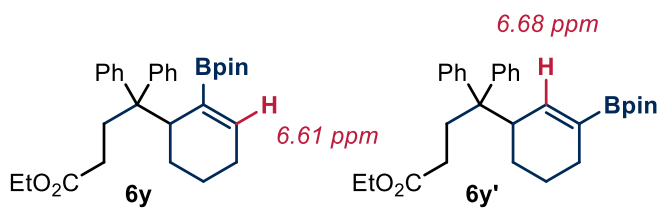
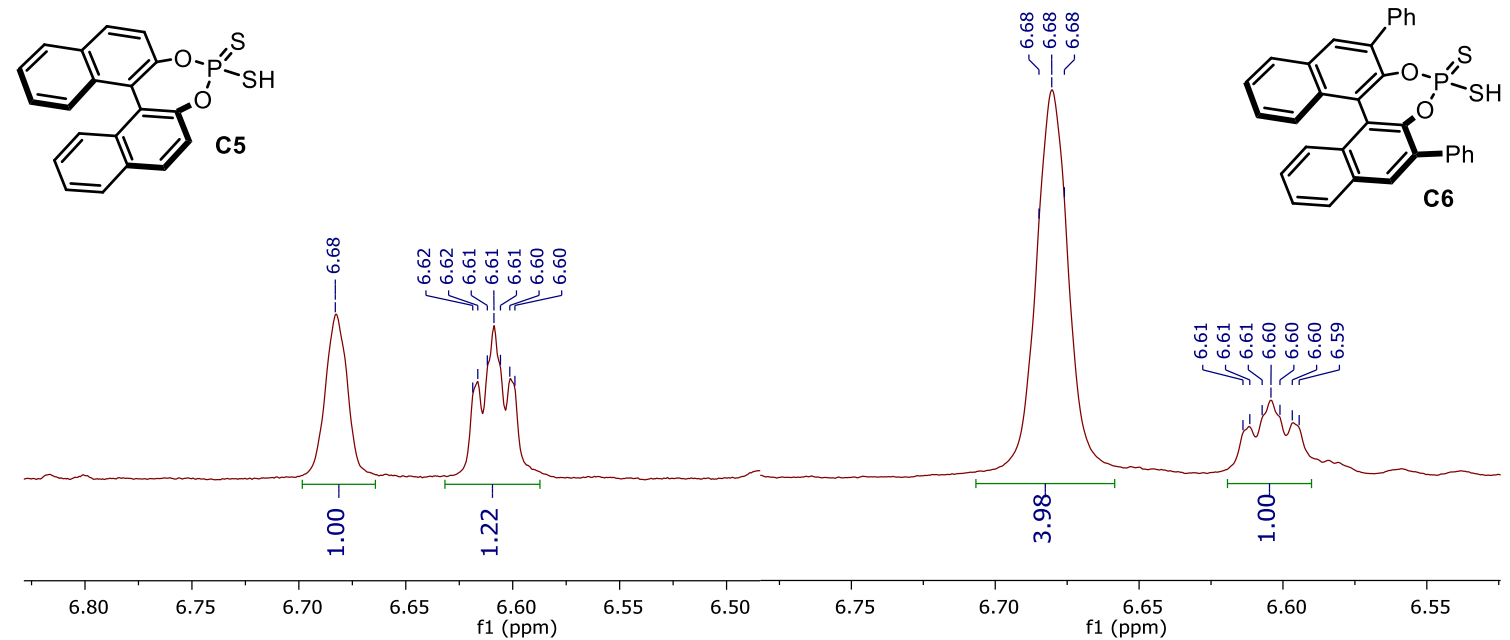

Figure S14. Regioselectivity study of 6y and 6y' using catalysts C5 and C6. 


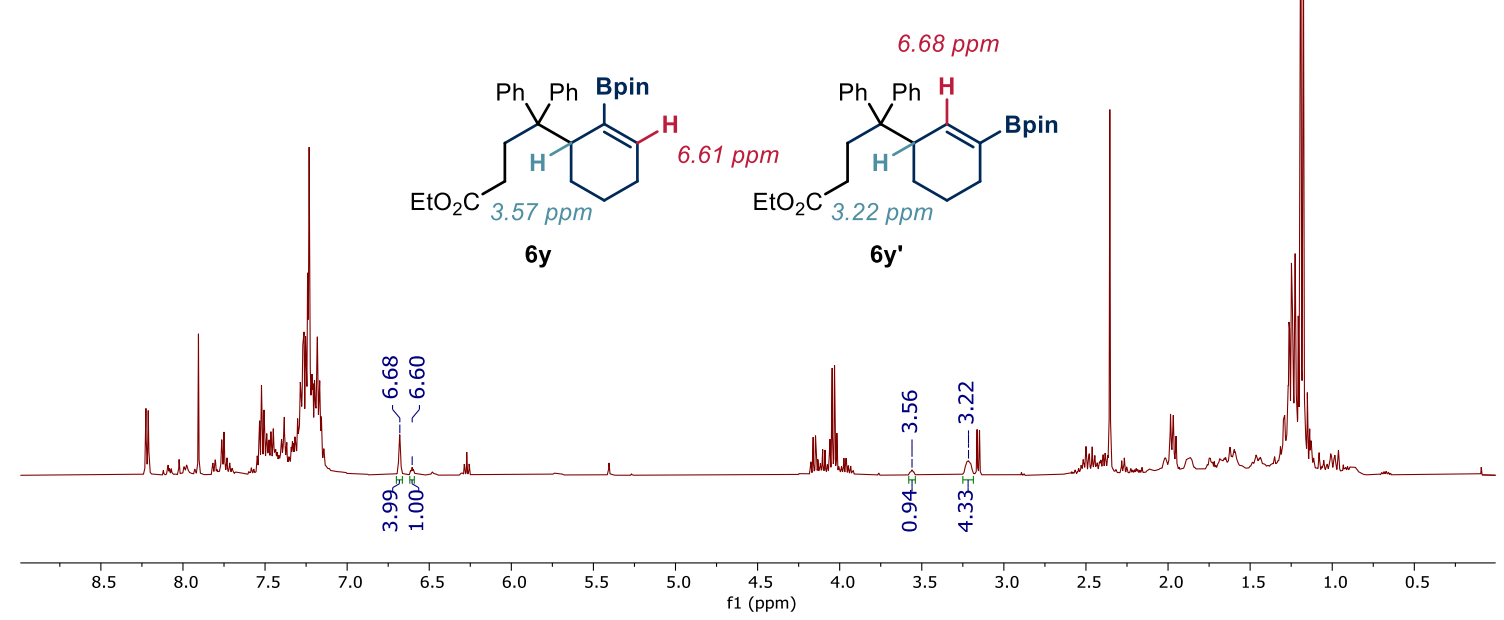

Figure S15. NMR spectrum of purified $\mathbf{6 y} / \mathbf{6 y}$ ', after column chromatography; reaction performed with catalyst $\mathbf{C 6}$.

\section{E.4 DFT calculations}

\section{Computational Methods}

Calculations were performed using the Gaussian 09 suite of programs. ${ }^{24}$ DFT was applied using B3LYP functional. ${ }^{25}$ The $6-31 \mathrm{G}(\mathrm{d}, \mathrm{p})$ basis $\mathrm{set}^{26}$ was employed for all atoms $(\mathrm{C}, \mathrm{H}, \mathrm{O}, \mathrm{S}$ and $\mathrm{P})$. Full geometry optimizations were carried out in acetone, through an implicit solvent SMD. ${ }^{27}$ The stationary points were characterized by vibrational analysis. Minima were identified by the presence of a full set of real frequencies. Reported energies are potential energies (E), enthalpies $(\mathrm{H})$ and free energies $(\mathrm{G})$ in solution, computed at $298 \mathrm{~K}$ and $1 \mathrm{~atm}$. Homolytic bond dissociation enthalpies (BDE) were also calculated at the same level of theory.

\section{Bond Dissociation Enthalpies}

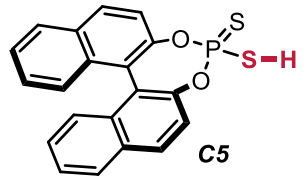

$\mathrm{BDE}(\mathrm{S}-\mathrm{H}): 83.3 \mathrm{kcal} \cdot \mathrm{mol}^{-1}$

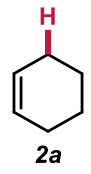

$\operatorname{BDE}(\mathrm{C}-\mathrm{H}): 82.2 \mathrm{kcal} \cdot \mathrm{mol}^{-1}$

Figure S15. Bond dissociation enthalpies (BDE) calculated for C5 and 2a. DFT Method: B3LYP/6-31G(d,p). 


\section{Computed Structures and Energies}

C5

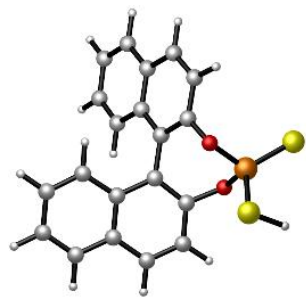

$E=-2161.232099$ Hartrees

$\mathrm{H}=-2160.934966$ Hartrees

$\mathrm{G}=-2161.003202$ Hartrees

$\mathrm{C}$

C $\quad-5.29970900$

C $\quad-6.06611800$

C $\quad-6.83161200$

C $\quad-6.84319300$

C $\quad-6.10978900$

$\mathrm{H}$

$\mathrm{H}$

$\mathrm{H}$

C

C

$\mathrm{H}$

$\mathrm{H}$

$\mathrm{H}$

C

C

$\mathrm{H}$

$\mathrm{H}$

C

C

C

C

C

C

C

$\mathrm{H}$

C

C

$\mathrm{H}$

C

$\mathrm{H}$

H

H

H

$\mathrm{O}$

$\mathrm{O}$

P

S

S

$\mathrm{H}$

$\begin{array}{llll}-4.72372500 & 5.51335700 & -5.29576000\end{array}$

$\begin{array}{llll}-4.68096300 & 3.98736100 & -3.38161400\end{array}$

$\begin{array}{llll}-6.06968700 & 4.26656200 & -1.03037600\end{array}$

$\begin{array}{llll}-7.54804000 & 6.69538900 & -0.95044100\end{array}$

$\begin{array}{llll}-7.43690100 & 8.12401500 & -3.21618200\end{array}$

$\begin{array}{llll}-6.12204400 & 7.57727400 & -5.24461800\end{array}$

$\begin{array}{lll}-7.48390000 & 5.89340200 & 0.16188400\end{array}$

$\begin{array}{llll}-6.73477700 & 4.70141200 & 0.10501500\end{array}$

$\begin{array}{llll}-8.12880300 & 7.61270400 & -0.93183300\end{array}$

$\begin{array}{llll}-7.99723300 & 6.14608900 & 1.08293400\end{array}$

$\begin{array}{llll}-5.36678400 & 2.95333900 & -1.01878000\end{array}$

$\begin{array}{llll}-5.63083800 & 1.91649600 & -1.98106000\end{array}$

$\begin{array}{llll}-4.43773100 & 2.68209700 & -0.02804200\end{array}$

$\begin{array}{llll}-6.66188200 & 2.01791600 & -2.95668800\end{array}$

$\begin{array}{llll}-4.85326200 & 0.70970800 & -1.94274100\end{array}$

$\begin{array}{llll}-3.67920200 & 1.49690800 & 0.03073400\end{array}$

$\begin{array}{llll}-6.87990200 & 1.00714200 & -3.86686100\end{array}$

$\begin{array}{llll}-7.29068300 & 2.89964700 & -2.97655900\end{array}$

$\begin{array}{llll}-5.09555500 & -0.30729800 & -2.90626500\end{array}$

$\begin{array}{llll}-3.87176800 & 0.53537200 & -0.93051600\end{array}$

$\begin{array}{llll}-2.95724100 & 1.37511400 & 0.83044200\end{array}$

$\begin{array}{lll}-6.08252000 & -0.16227200 & -3.85396800\end{array}$

$\begin{array}{llll}-7.67617400 & 1.10637300 & -4.59869500\end{array}$

$\begin{array}{llll}-4.49048100 & -1.20918900 & -2.87001400\end{array}$

$\begin{array}{llll}-3.28974700 & -0.38118900 & -0.91234800\end{array}$

$\begin{array}{lll}-6.26206500 & -0.94704800 & -4.58267600\end{array}$

$\begin{array}{llll}-6.71400100 & 3.91732700 & 1.27068200\end{array}$

$\begin{array}{llll}-4.19106000 & 3.64340000 & 0.96887800\end{array}$

$\begin{array}{llll}-5.32693900 & 3.81594700 & 2.13834500\end{array}$

$\begin{array}{llll}-5.48240800 & 2.45239400 & 3.50841800\end{array}$

$\begin{array}{llll}-4.73147800 & 5.76338200 & 2.70105200\end{array}$

$\begin{array}{llll}-5.40334500 & 5.72598300 & 3.87253800\end{array}$ 


\section{II}

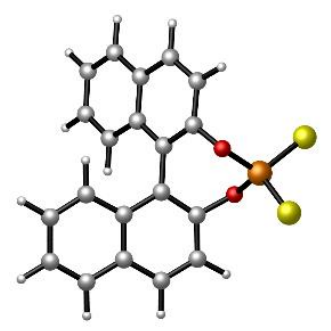

$E=-2160.567518$ Hartrees

$\mathrm{H}=-2160.279672$ Hartrees

$\mathrm{G}=-2160.348175$ Hartrees

$\mathrm{C}$

$\begin{array}{llll}-5.34657200 & 5.73580500 & -4.45150200\end{array}$

$\begin{array}{llll}\text { C } & -5.32032500 & 4.87473600 & -3.37655300\end{array}$

C $\quad-6.07300500 \quad 5.14813500 \quad-2.20036700$

C $\quad-6.82735100 \quad 6.36909400 \quad-2.14843800$

$\begin{array}{llll}\text { C } & -6.84318800 & 7.22789500 & -3.28151500\end{array}$

C $\quad-6.12396700 \quad 6.91810400 \quad-4.41280900$

$\mathrm{H} \quad-4.75935600 \quad 5.50764600 \quad-5.33613800$

$\mathrm{H} \quad-4.70922100 \quad 3.98151900 \quad-3.42202300$

$\begin{array}{llll}\text { C } & -6.07221900 & 4.27413800 & -1.05718300\end{array}$

C $\quad-7.52708800 \quad 6.71616600 \quad-0.96207200$

$\mathrm{H} \quad-7.42822000 \quad 8.14204300 \quad-3.22944900$

$\mathrm{H} \quad-6.13917900 \quad 7.58318800 \quad-5.27106200$

$\begin{array}{llll}\text { C } & -7.46140400 & 5.91321900 & 0.15033300\end{array}$

C $\quad-6.72548900 \quad 4.71482200 \quad 0.08125000$

$\mathrm{H} \quad-8.09705200 \quad 7.64001500 \quad-0.93724500$

$\mathrm{H} \quad-7.96377000 \quad 6.16997600 \quad 1.07628900$

C $\quad-5.36612400 \quad 2.96341200 \quad-1.03813600$

C $\quad-5.62982400 \quad 1.91630200 \quad-1.98871500$

$\begin{array}{llll}\text { C } & -4.43455700 & 2.70405200 & -0.04699600\end{array}$

$\begin{array}{llll}\text { C } & -6.66181100 & 2.00683700 & -2.96418100\end{array}$

$\begin{array}{llll}\text { C } & -4.85096000 & 0.71074500 & -1.93775300\end{array}$

C $\quad-3.67555700 \quad 1.52041700 \quad 0.02631900$

C $\quad-6.87921000 \quad 0.98615300 \quad-3.86336800$

$\mathrm{H} \quad-7.29138600 \quad 2.88779600 \quad-2.99300300$

C $\quad \begin{array}{llll}-5.09273700 & -0.31665400 & -2.89038500\end{array}$

C $\quad-3.86945000 \quad 0.54776000 \quad-0.92400400$

$\mathrm{H} \quad-2.95136800 \quad 1.40823600 \quad 0.82543500$

C $\quad-6.08058500 \quad-0.18226800 \quad-3.83864100$

$\mathrm{H} \quad-7.67597700 \quad 1.07672100 \quad-4.59576000$

$\mathrm{H} \quad-4.48686700 \quad-1.21754500 \quad-2.84503400$

$\mathrm{H} \quad-3.28715300 \quad-0.36831100 \quad-0.89574500$

$\mathrm{H} \quad-6.25996500 \quad-0.97468000 \quad-4.55905000$

$\begin{array}{llll}\mathrm{O} & -6.69656100 & 3.92246700 & 1.24915600\end{array}$

$\begin{array}{llll}\mathrm{O} & -4.18224300 & 3.67897200 & 0.94179000\end{array}$

$\begin{array}{llll}\mathrm{P} & -5.30760400 & 3.88867200 & 2.10323100\end{array}$

$\begin{array}{llll}\mathrm{S} & -5.43592700 & 2.44224500 & 3.47018600\end{array}$

S $\quad-4.86621600 \quad 5.54444700 \quad 3.12372000$ 


\section{Hydrogen}

$\mathrm{E}=-0.5248775$ Hartrees

$\mathrm{H}=-0.5225518$ Hartrees

$\mathrm{G}=-0.535532$ Hartrees

$\begin{array}{llll}\mathrm{H} & 0.00000000 & 0.00000000 & 0.00000000\end{array}$

$2 \mathbf{a}$

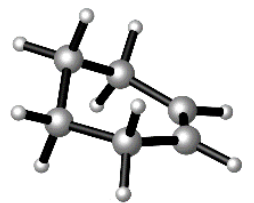

$E=-234.679273$ Hartrees

$\mathrm{H}=-234.526666$ Hartrees

$\mathrm{G}=-234.561708$ Hartrees

$\begin{array}{llll}\text { C } & -2.10823400 & -0.79654900 & 0.02049100\end{array}$

$\begin{array}{llll}\text { C } & -0.58334400 & -0.94771500 & 0.13952600\end{array}$

$\begin{array}{llll}\mathrm{C} & 0.07641800 & 0.32265000 & 0.61942900\end{array}$

$\begin{array}{llll}\text { C } & -0.59660600 & 1.34634000 & 1.15784700\end{array}$

$\begin{array}{llll}\text { C } & -2.09000800 & 1.33617200 & 1.37816700\end{array}$

$\begin{array}{llll}\text { C } & -2.68178100 & -0.07605200 & 1.24806700\end{array}$

$\mathrm{H} \quad 1.15857900 \quad 0.38860900 \quad 0.51068900$

$\mathrm{H} \quad-0.33903600 \quad-1.76736900 \quad 0.83306600$

$\mathrm{H} \quad-0.15282400-1.24433700 \quad-0.82617900$

$\mathrm{H} \quad-2.34404100 \quad-0.21145000 \quad-0.87886800$

$\mathrm{H} \quad-2.57670500 \quad-1.77919700 \quad-0.10769400$

$\mathrm{H} \quad-0.05782800 \quad 2.24311200 \quad 1.46235500$

$\mathrm{H} \quad-2.32028900 \quad 1.75613900 \quad 2.36620300$

$\mathrm{H} \quad-2.56845300 \quad 2.01302600 \quad 0.65345500$

$\mathrm{H} \quad-2.43608500 \quad-0.65507900 \quad 2.14880000$

$\mathrm{H} \quad-3.77527000 \quad-0.02410800 \quad 1.19208700$

IV

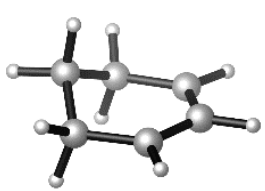

$\mathrm{E}=-234.036923$ Hartrees

$\mathrm{H}=-233.898149$ Hartrees

$\mathrm{G}=-233.933771$ Hartrees

$\begin{array}{lrcc}\mathrm{C} & -2.08793000 & -0.79340900 & 0.03581000 \\ \mathrm{C} & -0.56129400 & -0.95470700 & 0.14637100 \\ \mathrm{C} & 0.10125100 & 0.33826800 & 0.53495800 \\ \mathrm{C} & -0.59827800 & 1.31508400 & 1.23627900 \\ \mathrm{C} & -1.92209100 & 1.14600900 & 1.62849900 \\ \mathrm{C} & -2.67397400 & -0.11004400 & 1.28413900 \\ \mathrm{H} & 1.14701800 & 0.49216400 & 0.28179000 \\ \mathrm{H} & -0.32912400 & -1.72789700 & 0.89858400 \\ \mathrm{H} & -0.14722700 & -1.32876800 & -0.79850200 \\ \mathrm{H} & -2.31298100 & -0.17429700 & -0.84207900\end{array}$


$\mathrm{H} \quad-2.56389200 \quad-1.76683400 \quad-0.12521200$

$\begin{array}{llll}\mathrm{H} & -0.08580900 & 2.23859500 & 1.50177100\end{array}$

$\begin{array}{llll}\mathrm{H} & -2.42115800 & 1.91640100 & 2.21061200\end{array}$

$\mathrm{H} \quad-2.63309200 \quad-0.80943800 \quad 2.13640900$

$\begin{array}{lllll}\mathrm{H} & -3.73847200 & 0.11003800 & 1.13455600 .\end{array}$ 


\section{F. References}

1. Tanabe, S.; Mitsunuma, H.; Kanai, M. Catalytic Allylation of Aldehydes Using Unactivated Alkenes. J. Am. Chem. Soc. 2020, 142, 12374-12381.

2. Shapiro, N. D.; Rauniyar, V.; Hamilton, G. L.; Wu, J.; Toste, F. D. Asymmetric Additions to Dienes Catalysed by a Dithiophosphoric Acid. Nature. 2011, 470, 245-249.

3. Fernandes, A.; Laye, C.; Pramanik, S.; Palmeira, D.; Pekel, Ö. Ö.; Massip, S.; Schmidtmann, M.; Müller, T.; Robert, F.; Landais, Y. Chiral Memory in Silyl-Pyridinium and Quinolinium Cations. J. Am. Chem. Soc. 2020, 142, 564-572.

4. Horn, E. J.; Rosen, B. R.; Chen, Y.; Tang, J.; Chen, K.; Eastgate, M. D.; Baran, P. S. Scalable and Sustainable Electrochemical Allylic C-H Oxidation. Nature 2016, 533, 77-81.

5. Leibler, I.; Tekle-Smith, M. A.; Doyle, A. A General Strategy for C(Sp3)-H Functionalization with Nucleophiles Using Methyl Radical as a Hydrogen Atom Abstractor. 2021. https://doi.org/10.26434/CHEMRXIV.13644218.V1.

6. Qin, T.; Cornella, J.; Li, C.; Malins, L. R.; Edwards, J. T.; Kawamura, S.; Maxwell, B. D.; Eastgate, M. D.; Baran, P. S. Organic Chemistry: A General Alkyl-Alkyl Cross-Coupling Enabled by Redox-Active Esters and Alkylzinc Reagents. Science, 2016, 352, 801-805.

7. Ni, S.; Garrido-Castro, A. F.; Merchant, R. R.; de Gruyter, J. N.; Schmitt, D. C.; Mousseau, J. J.; Gallego, G. M.; Yang, S.; Collins, M. R.; Qiao, J. X.; Yeung, K. S.; Langley, D. R.; Poss, M. A.; Scola, P. M.; Qin, T.; Baran, P. S. A General Amino Acid Synthesis Enabled by Innate Radical Cross-Coupling. Angew. Chem., Int. Ed. 2018, 57, 14560-14565.

8. Chen, T. G.; Zhang, H.; Mykhailiuk, P. K.; Merchant, R. R.; Smith, C. A.; Qin, T.; Baran, P. S. Quaternary Centers by Nickel-Catalyzed Cross-Coupling of Tertiary Carboxylic Acids and (Hetero)Aryl Zinc Reagents. Angew. Chem., Int. Ed. 2019, 58, 2454-2458.

9. Zeng, X.; Yan, W.; Zacate, S. B.; Chao, T. H.; Sun, X.; Cao, Z.; Bradford, K. G. E.; Paeth, M.; Tyndall, S. B.; Yang, K.; Kuo, T. C.; Cheng, M. J.; Liu, W. Copper-Catalyzed Decarboxylative Difluoromethylation. J. Am. Chem. Soc. 2019, 141, 11398-11403.

10. Bosque, I.; Bach, T. 3-Acetoxyquinuclidine as Catalyst in Electron Donor-Acceptor Complex-Mediated Reactions Triggered by Visible Light. ACS Catal. 2019, 9, 9103-9109.

11. Klauck, F. J. R.; Yoon, H.; James, M. J.; Lautens, M.; Glorius, F. Visible-Light-Mediated Deaminative Three-Component Dicarbofunctionalization of Styrenes with Benzylic Radicals. ACS Catal. 2019, 9, 236-241.

12. James, M. J.; Strieth-Kalthoff, F.; Sandfort, F.; Klauck, F. J. R.; Wagener, F.; Glorius, F. Visible-Light-Mediated Charge Transfer Enables C-C Bond Formation with Traceless Acceptor Groups. Chem. - A Eur. J. 2019, 25, 8240-8244.

13. Zhang, C.-S.; Bao, L.; Chen, K.-Q.; Wang, Z.-X.; Chen, X.-Y. Photoinduced $\alpha$-Alkenylation of Katritzky Salts: Synthesis of $\beta, \gamma$-Unsaturated Esters. Org. Lett. 2021, 23, 1577-1581.

14. Laroche, B.; Tang, X.; Archer, G.; Di Sanza, R.; Melchiorre, P. Photochemical Chemoselective Alkylation of Tryptophan-Containing Peptides. Org. Lett. 2021, 23, 285-289.

15. Liao, J.; Guan, W.; Boscoe, B. P.; Tucker, J. W.; Tomlin, J. W.; Garnsey, M. R.; Watson, M. P. Transforming Benzylic Amines into Diarylmethanes: Cross-Couplings of Benzylic Pyridinium Salts via C-N Bond Activation. Org. Lett. 2018, 20, 3030-3033.

16. Wan, C.; Song, R.-J.; Li, J.-H. Electrooxidative 1,2-Bromoesterification of Alkenes with Acids and N -Bromosuccinimide. Org. Lett. 2019, 21, 2800-2803. 
17. Beck, R.; Camadanli, S.; Flörke, U.; Klein, H.-F. Reactivity Diversification - Synthesis and Exchange Reactions of Cobalt and Iron 2-Alkenylpyridine/-Pyrazine Complexes Obtained by Vinylic C(Sp2)-H Activation. Eur. J. Inorg. Chem. 2015, 2015, 2543-2559.

18. Chatalova-Sazepin, C.; Wang, Q.; Sammis, G. M.; Zhu, J. Copper-Catalyzed Intermolecular Carboetherification of Unactivated Alkenes by Alkyl Nitriles and Alcohols. Angew. Chem., Int. Ed. 2015, 54, 5443-5446.

19. Ritz, M. D.; Parsons, A. M.; Palermo, P. N.; Jones, W. D. Bisoxazoline-Pincer Ligated CobaltCatalyzed Hydrogenation of Alkenes. Polyhedron 2020, 180, 114416.

20. Tang, J.; Hackenberger, D.; Goossen, L. J. Branched Arylalkenes from Cinnamates: Selectivity Inversion in Heck Reactions by Carboxylates as Deciduous Directing Groups. Angew. Chem., Int. Ed. 2016, 55 , 11296-11299.

21. Milan, M.; Carboni, G.; Salamone, M.; Costas, M.; Bietti, M. Tuning Selectivity in Aliphatic $\mathrm{C}-\mathrm{H}$ Bond Oxidation of $\mathrm{N}$-Alkylamides and Phthalimides Catalyzed by Manganese Complexes. ACS Catal. 2017, 7, 5903-5911.

22. Zhang, J.; Stanciu, C.; Wang, B.; Hussain, M. M.; Da, C.; Carroll, P. J.; Dreher, S. D.; Walsh, P. J. Palladium-Catalyzed Allylic Substitution with $\left(\eta^{6}\right.$-Arene- $\left.\mathrm{CH}_{2} \mathrm{Z}\right) \mathrm{Cr}(\mathrm{CO})_{3}$-Based Nucleophiles. J. Am. Chem. Soc. 2011, 133, 20552-20560.

23. Thompson, J. L. T.; Davies, H. M. L., Enhancement of Cyclopropanation Chemistry in the Silver-Catalyzed Reactions of Aryldiazoacetates. J. Am. Chem. Soc. 2007, 129, 6090-6091.

24. Gaussian 09, Revision B.1, Frisch, M. J., Trucks, G. W., Schlegel, H. B., Scuseria, G. E., Robb, M. A., Cheeseman, J. R., Scalmani, G., Barone, V., Mennucci, B., Petersson, G. A., Nakatsuji, H., Caricato, M., Li, X., Hratchian, H. P., Izmaylov, A. F., Bloino, J., Zheng, G., Sonnenberg, J. L., Hada, M., Ehara, M., Toyota, K., Fukuda, R., Hasegawa, J., Ishida, M., Nakajima, T., Honda, Y., Kitao, O., Nakai, H., Vreven, T., Montgomery, J. A., Peralta, Jr. J. E., Ogliaro, F., Bearpark, M., Heyd, J. J., Brothers, E., Kudin, K. N., Staroverov, V. N., Kobayashi, R., Normand, J., Raghavachari, K., Rendell, A., Burant, J. C., Iyengar, S. S., Tomasi, J., Cossi, M., Rega, N., Millam, J. M., Klene, M., Knox, J. E., Cross, J. B., Bakken, V., Adamo, C., Jaramillo, J., Gomperts, R., Stratmann, R. E., Yazyev, O., Austin, A. J., Cammi, R., Pomelli, C., Ochterski, J. W., Martin, R.L., Morokuma, K.,, Zakrzewski, V. G., Voth, G. A., Salvador, P., Dannenberg, J. J., Dapprich, S., Daniels, A. D., Farkas, Ö., Foresman, J. B., Ortiz, J. V., Cioslowski, J., Fox, D. J. Gaussian, Inc., Wallingford CT 2009.

25. Becke, A. D. Density-functional Thermochemistry. III. The Role of Exact Exchange. $J$. Chem. Phys. 1993, 98, 5648-5652.

26. Hehre, W. J.; Ditchfield, R.; Pople, J. A. Self-Consistent Molecular Orbital Methods. XII. Further Extensions of Gaussian-Type Basis Sets for Use in Molecular Orbital Studies of Organic Molecules. J. Chem. Phys. 1972, 56, 2257-2261.

27. Marenich, A. V.; Cramer, C. J.; Truhlar, D. G. Universal Solvation Model Based on Solute Electron Density and on a Continuum Model of the Solvent Defined by the Bulk Dielectric Constant and Atomic Surface Tensions. J. Phys. Chem. B 2009, 113, 6378-6396. 


\section{G. NMR Spectra}

${ }^{1} \mathrm{H}$ NMR (400 MHz, $\mathrm{CDCl}_{3}$ ) of $\mathbf{1 a}$<smiles>O=C(Cc1ccc(C(F)(F)F)cc1)N1C(=O)c2c(Cl)c(Cl)c(Cl)c(Cl)c2C1=O</smiles>

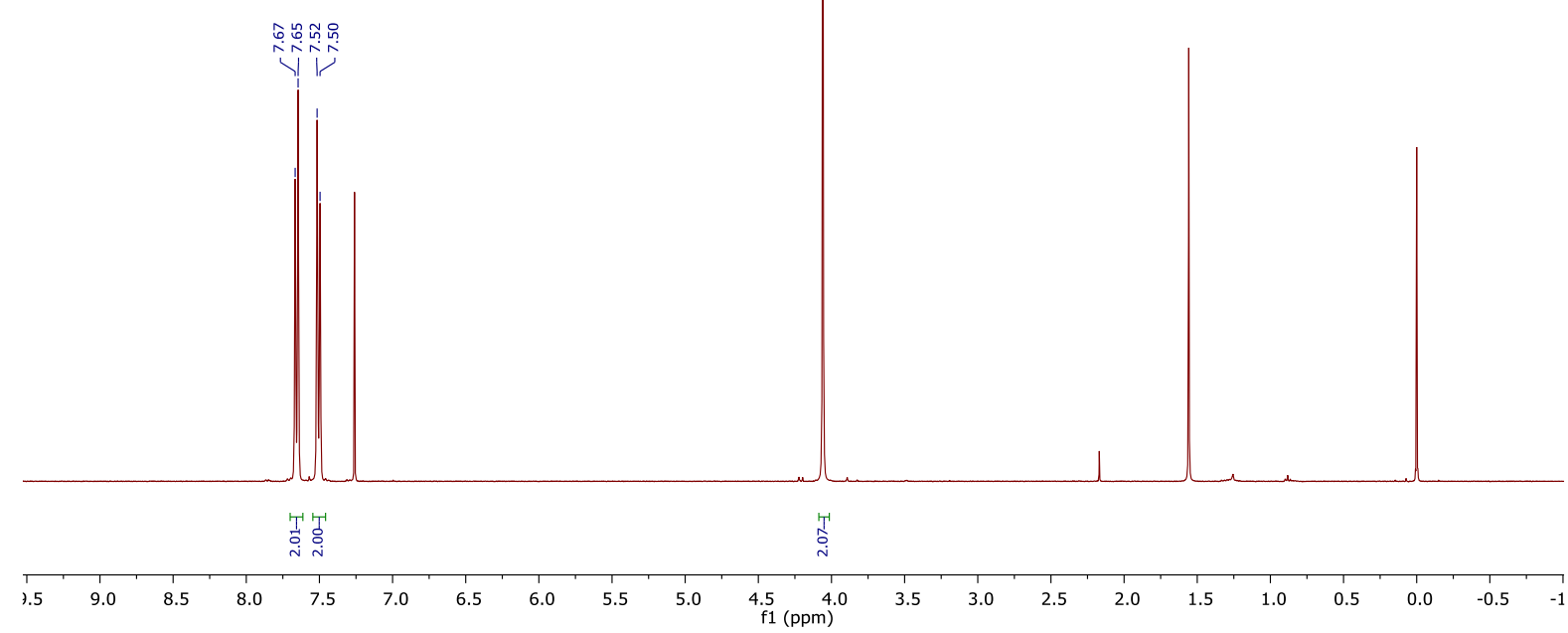

${ }^{13} \mathrm{C}$ NMR (101 MHz, $\mathrm{CDCl}_{3}$ ) of $\mathbf{1 a}$<smiles>O=C(Cc1ccc(C(F)(F)F)cc1)N1C(=O)c2c(Cl)c(Cl)c(Cl)c(Cl)c2C1=O</smiles>

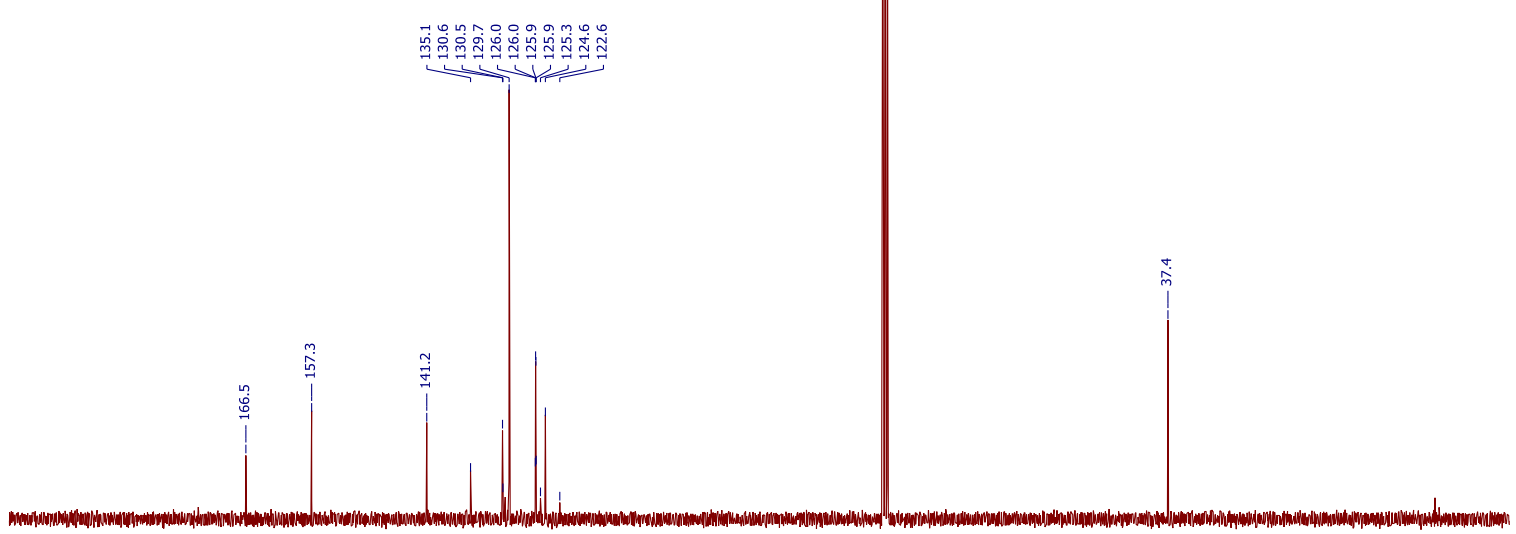

$\begin{array}{llllllllll}190 & 180 & 170 & 160 & 150 & 140 & 130 & 120 & 110 & \begin{array}{l}100 \\ \mathrm{f} 1(\mathrm{ppm})\end{array}\end{array}$

${ }^{19} \mathrm{~F} \mathrm{NMR}\left(376 \mathrm{MHz}, \mathrm{CDCl}_{3}\right)$ of $\mathbf{1 a}$ 


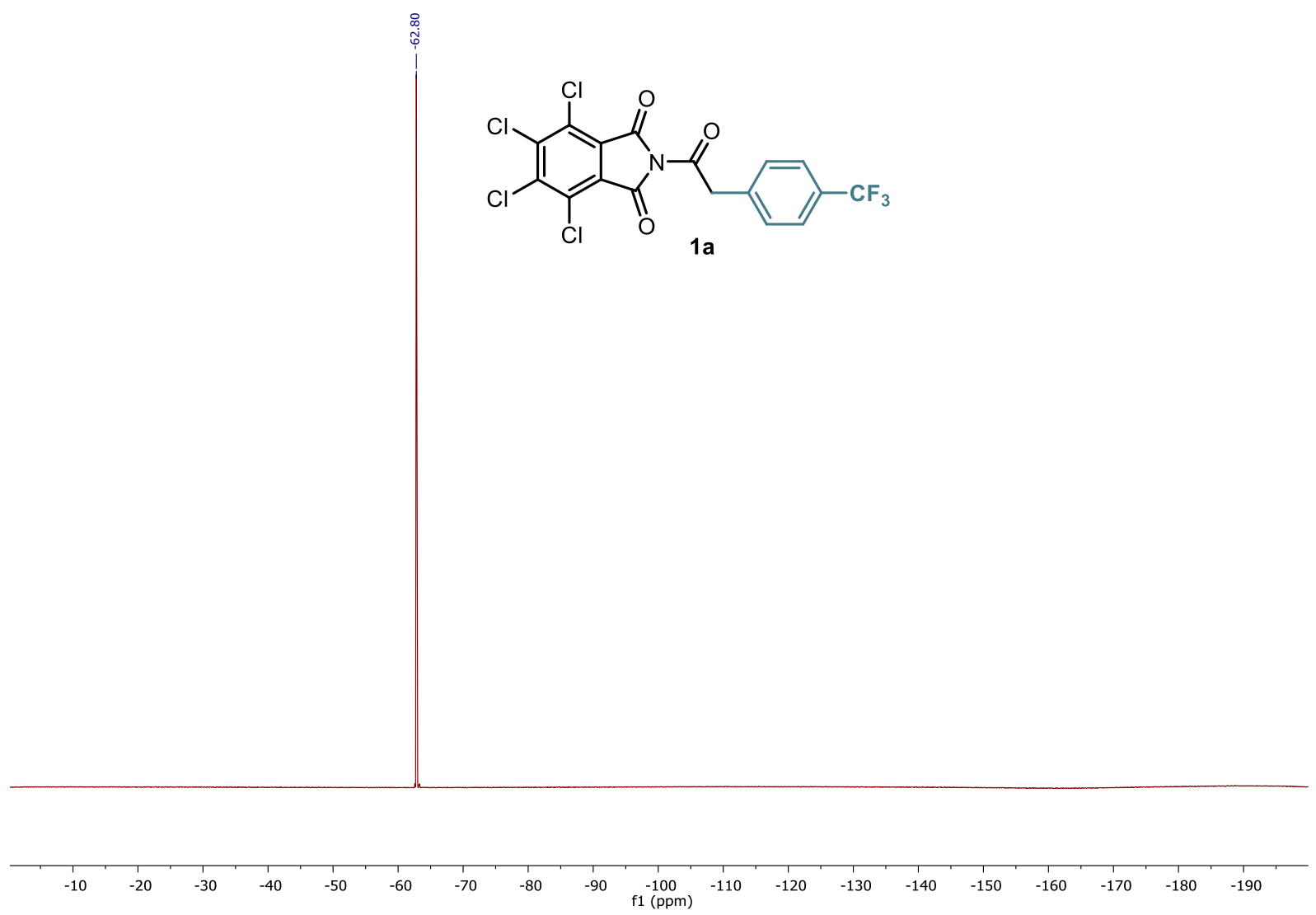

S48 
${ }^{1} \mathrm{H}$ NMR (400 MHz, $\mathrm{CDCl}_{3}$ ) of $\mathbf{1 b}$

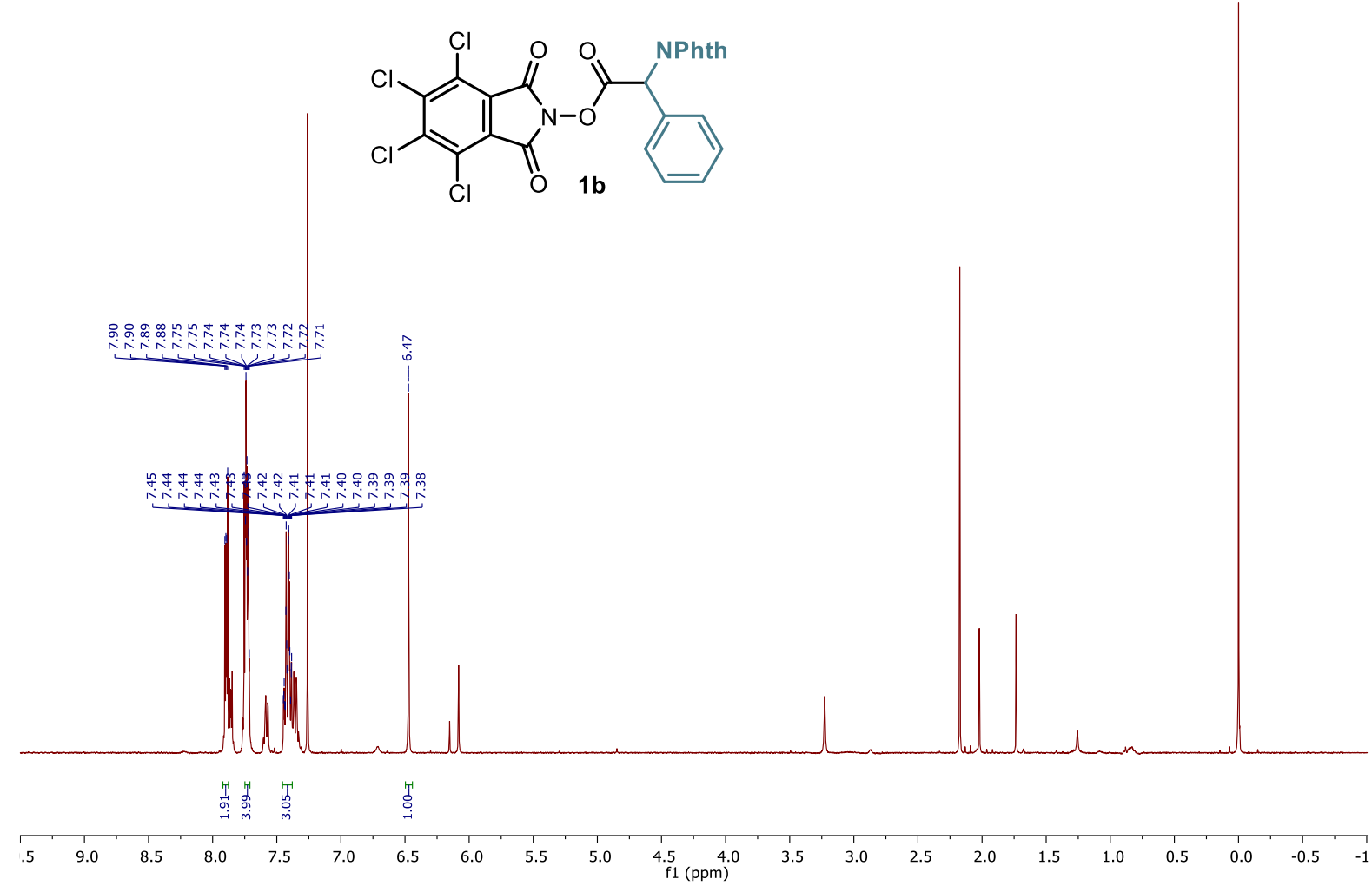

${ }^{13} \mathrm{C}$ NMR $\left(101 \mathrm{MHz}, \mathrm{CDCl}_{3}\right)$ of $\mathbf{1 b}$<smiles>O=C(ON1C(=O)c2c(Cl)c(Cl)c(Cl)c(Cl)c2C1=O)C(c1ccccc1)c1ccccc1</smiles>

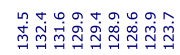
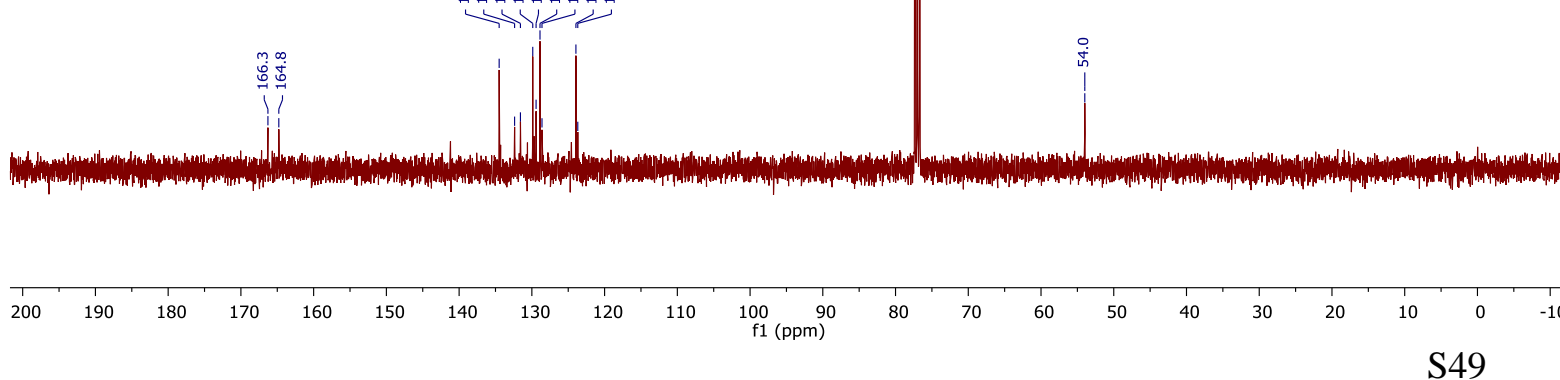
${ }^{1} \mathrm{H}$ NMR $\left(400 \mathrm{MHz}, \mathrm{CDCl}_{3}\right)$ of $\mathbf{1 d}$<smiles>CC(C)(C(=O)ON1C(=O)c2c(Cl)c(Cl)c(Cl)c(Cl)c2C1=O)c1ccccc1</smiles>

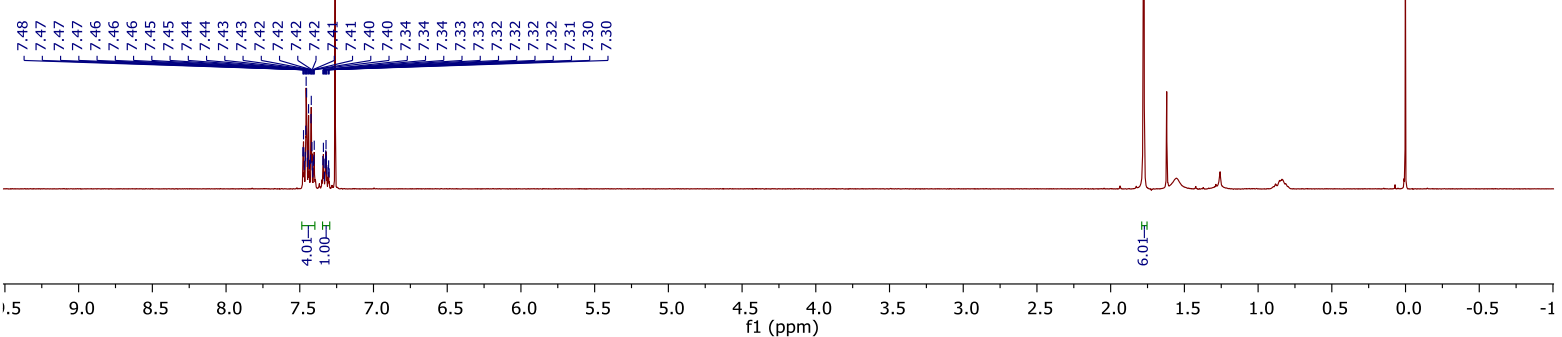

${ }^{13} \mathrm{C}$ NMR (101 MHz, $\mathrm{CDCl}_{3}$ ) of $\mathbf{1 d}$<smiles>CC(C)(C(=O)ON1C(=O)c2c(Cl)c(Cl)c(Cl)c(Cl)c2C1=O)c1ccccc1</smiles>

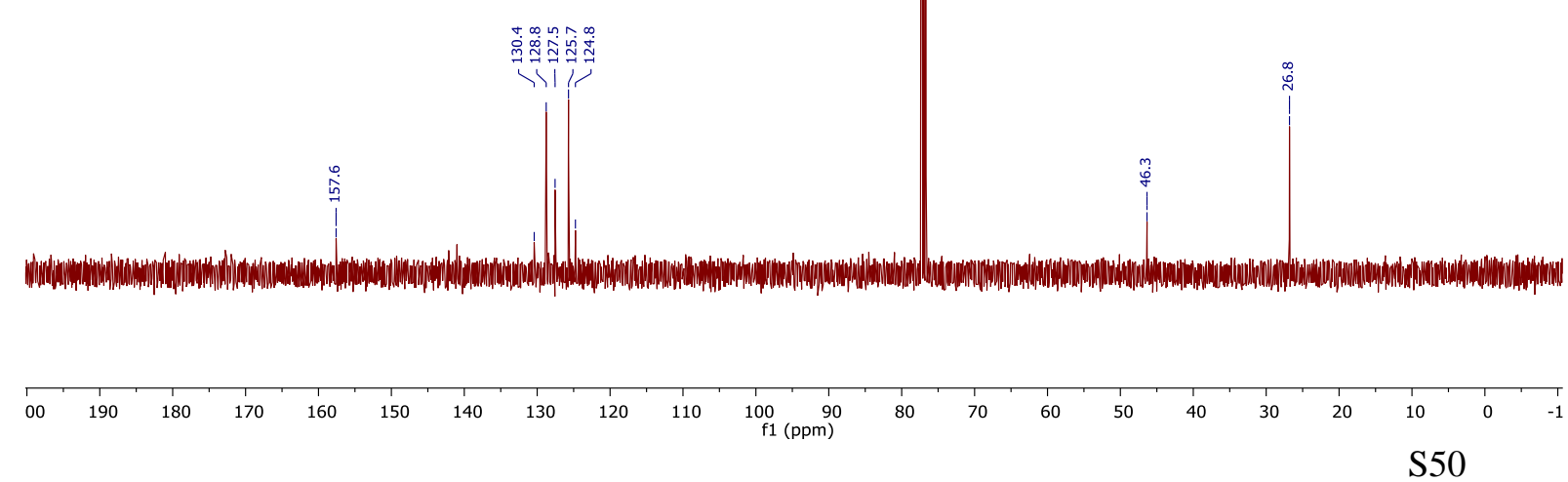


${ }^{1} \mathrm{H}$ NMR (400 MHz, $\mathrm{CDCl}_{3}$ ) of $\mathbf{1 e}$

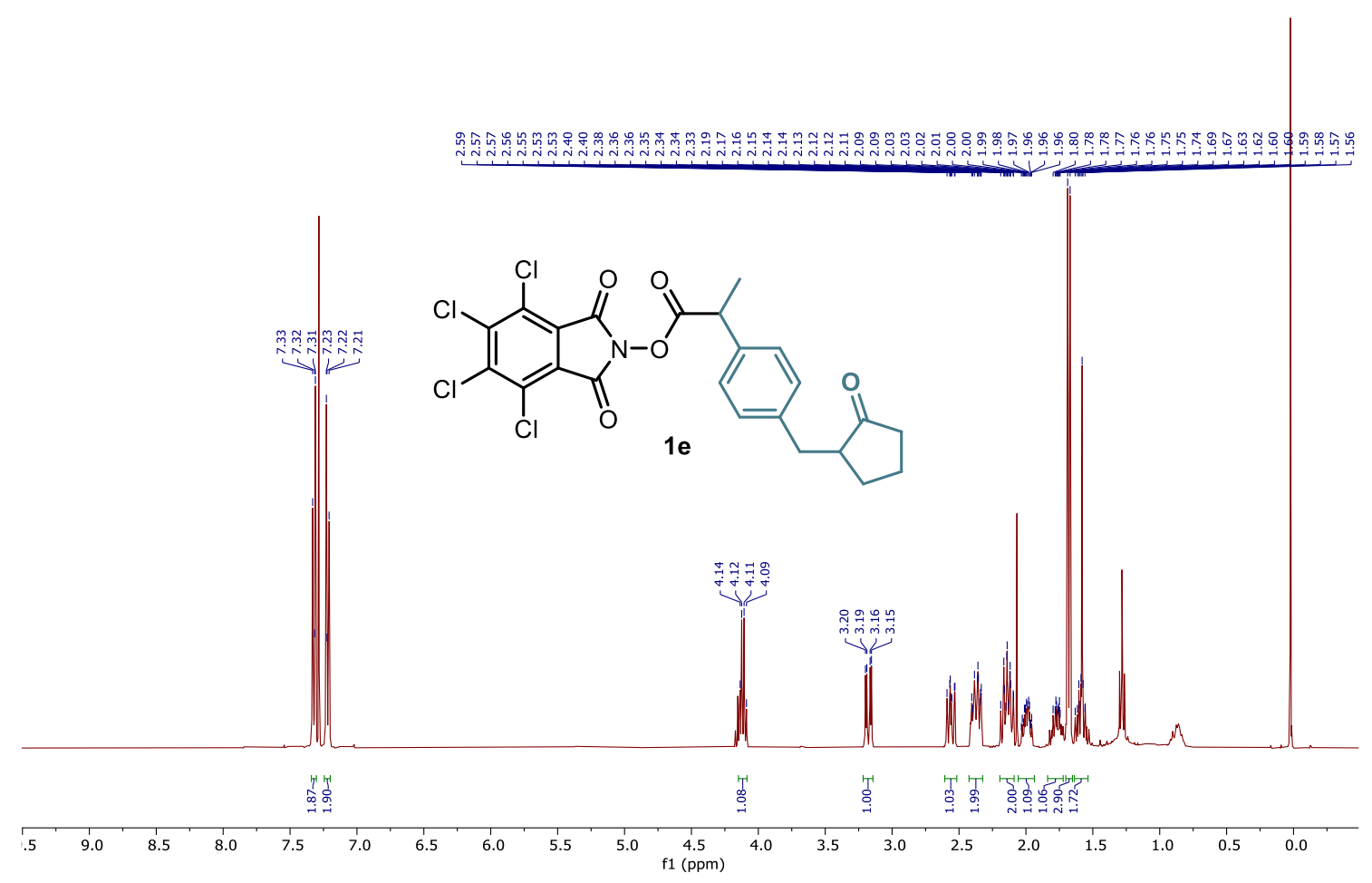

${ }^{13} \mathrm{C}$ NMR $\left(101 \mathrm{MHz}, \mathrm{CDCl}_{3}\right)$ of $\mathbf{1 e}$
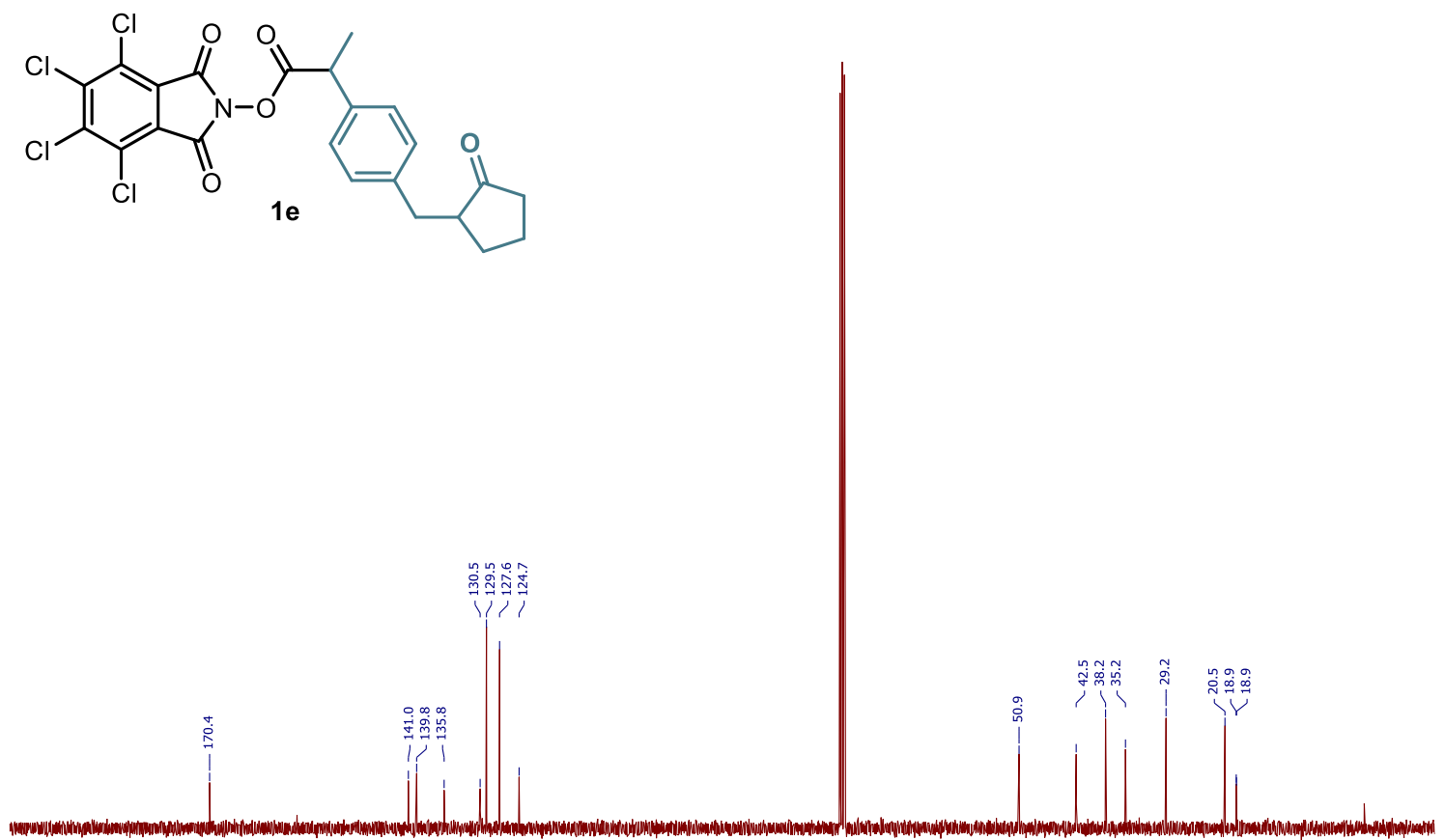

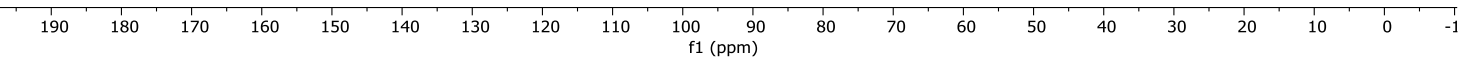


${ }^{1} \mathrm{H}$ NMR (400 MHz, $\mathrm{CDCl}_{3}$ ) of $\mathbf{1 g}$

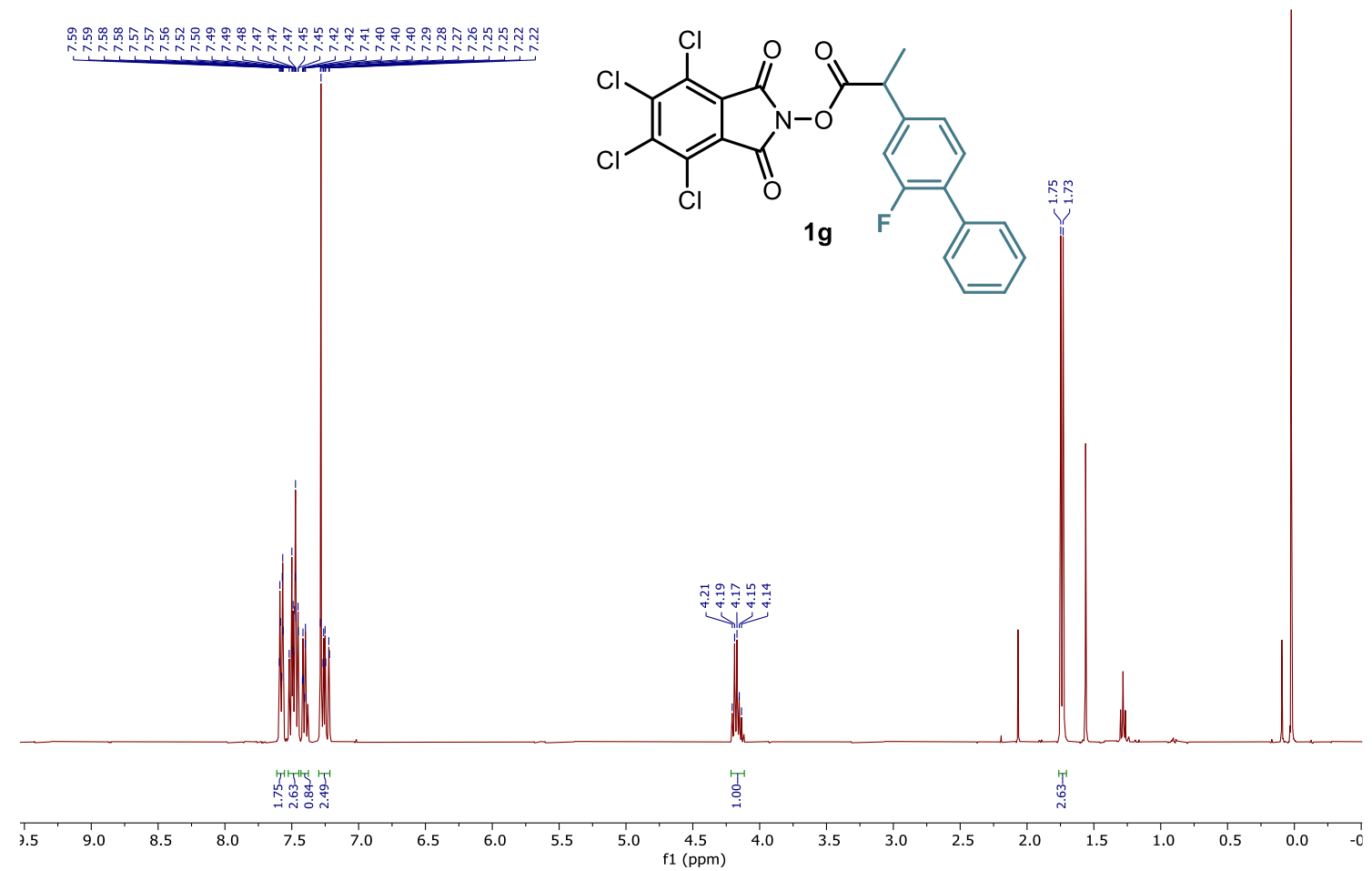

${ }^{13} \mathrm{C}$ NMR $\left(101 \mathrm{MHz}, \mathrm{CDCl}_{3}\right)$ of $\mathbf{1 g}$

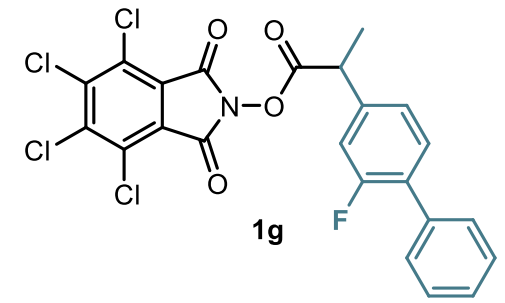

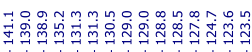

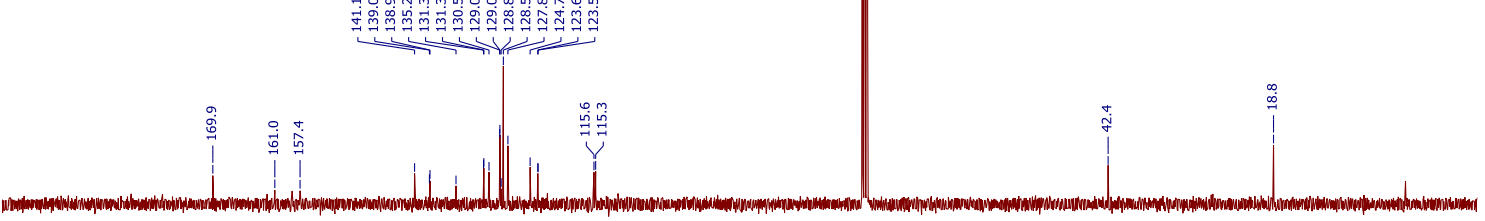

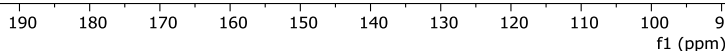


${ }^{19} \mathrm{~F}$ NMR $\left(376 \mathrm{MHz}, \mathrm{CDCl}_{3}\right)$ of $\mathbf{1 g}$
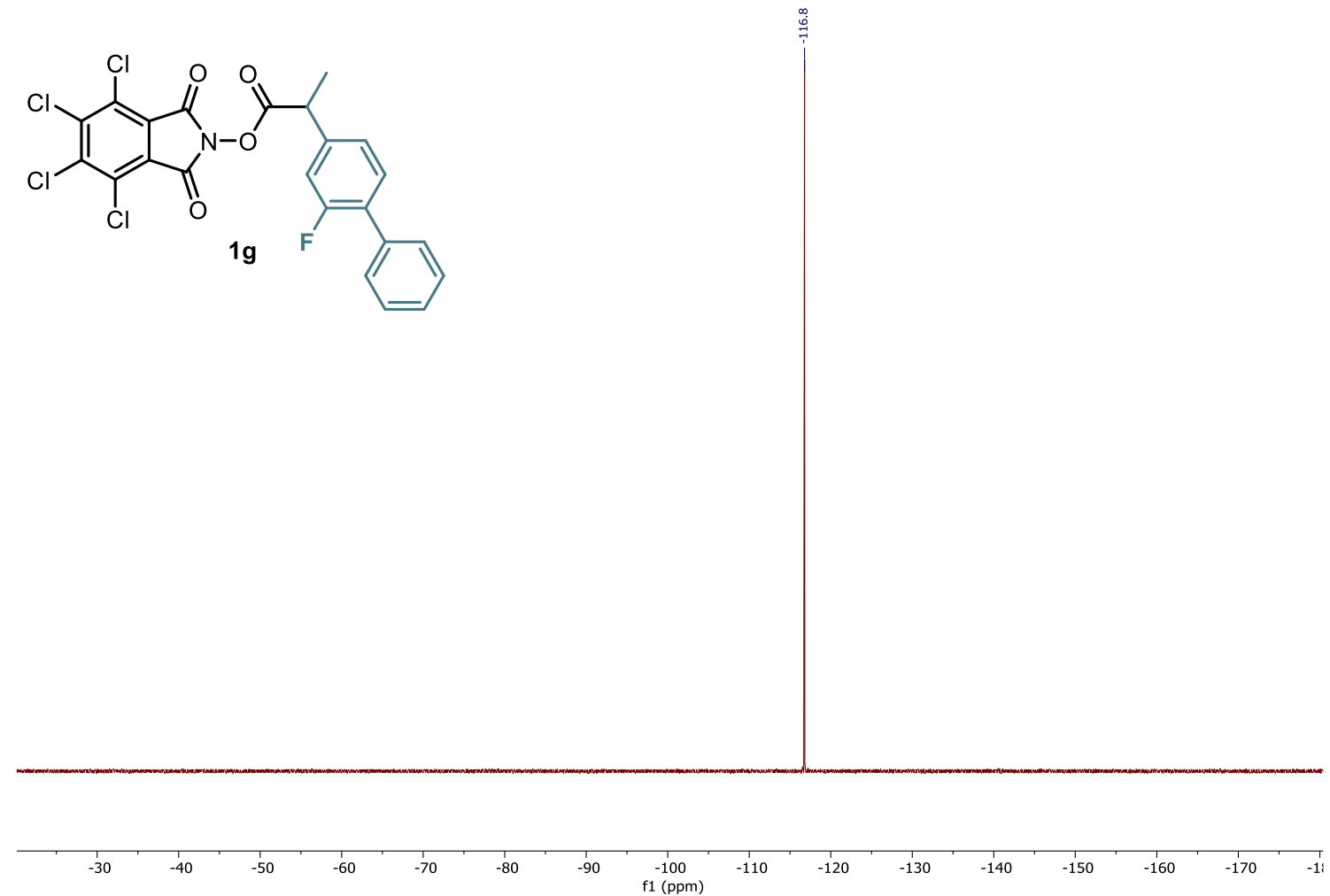
${ }^{1} \mathrm{H}$ NMR (400 MHz, $\mathrm{CDCl}_{3}$ ) of $\mathbf{1 n}$<smiles>CC(C)(C)NCCCC(=O)ON1C(=O)c2c(Cl)c(Cl)c(Cl)c(Cl)c2C1=O</smiles>

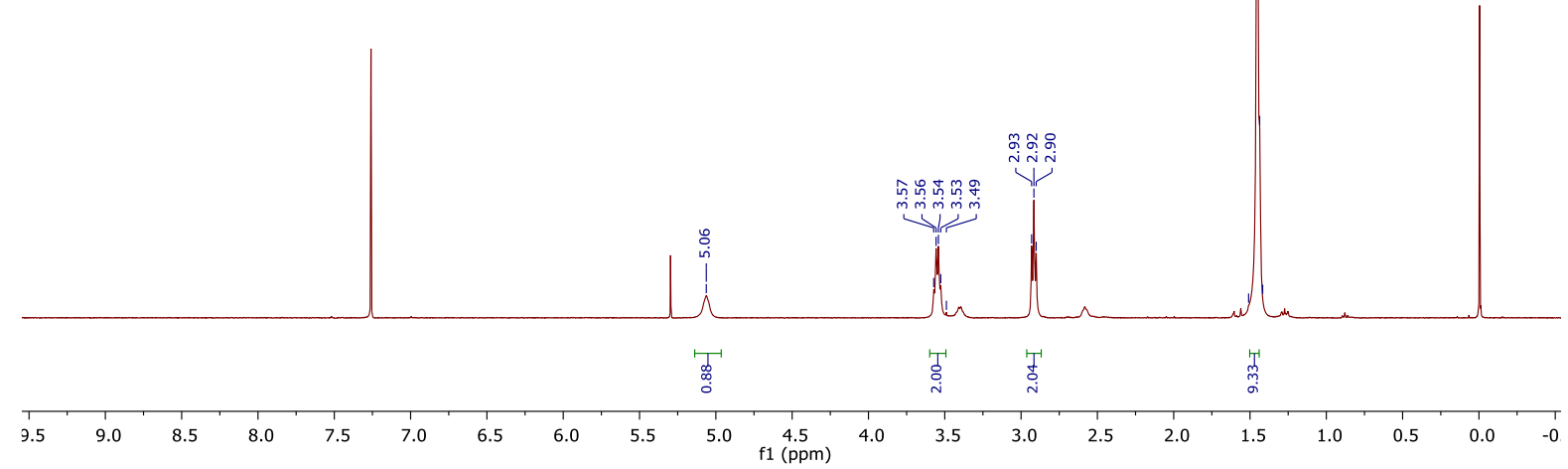

${ }^{13} \mathrm{C}$ NMR (101 MHz, $\mathrm{CDCl}_{3}$ ) of $\mathbf{1 n}$<smiles>CC(C)(C)NCCCC(=O)ON1C(=O)c2c(Cl)c(Cl)c(Cl)c(Cl)c2C1=O</smiles>

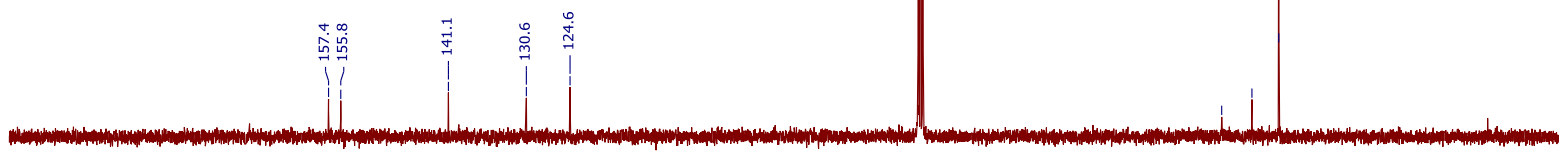

$\begin{array}{lllllllllll}200 & 190 & 180 & 170 & 160 & 150 & 140 & 130 & 120 & 110 & 100 \\ \text { f1 (ppm) }\end{array}$ 
${ }^{1} \mathrm{H}$ NMR (400 MHz, $\mathrm{CDCl}_{3}$ ) of

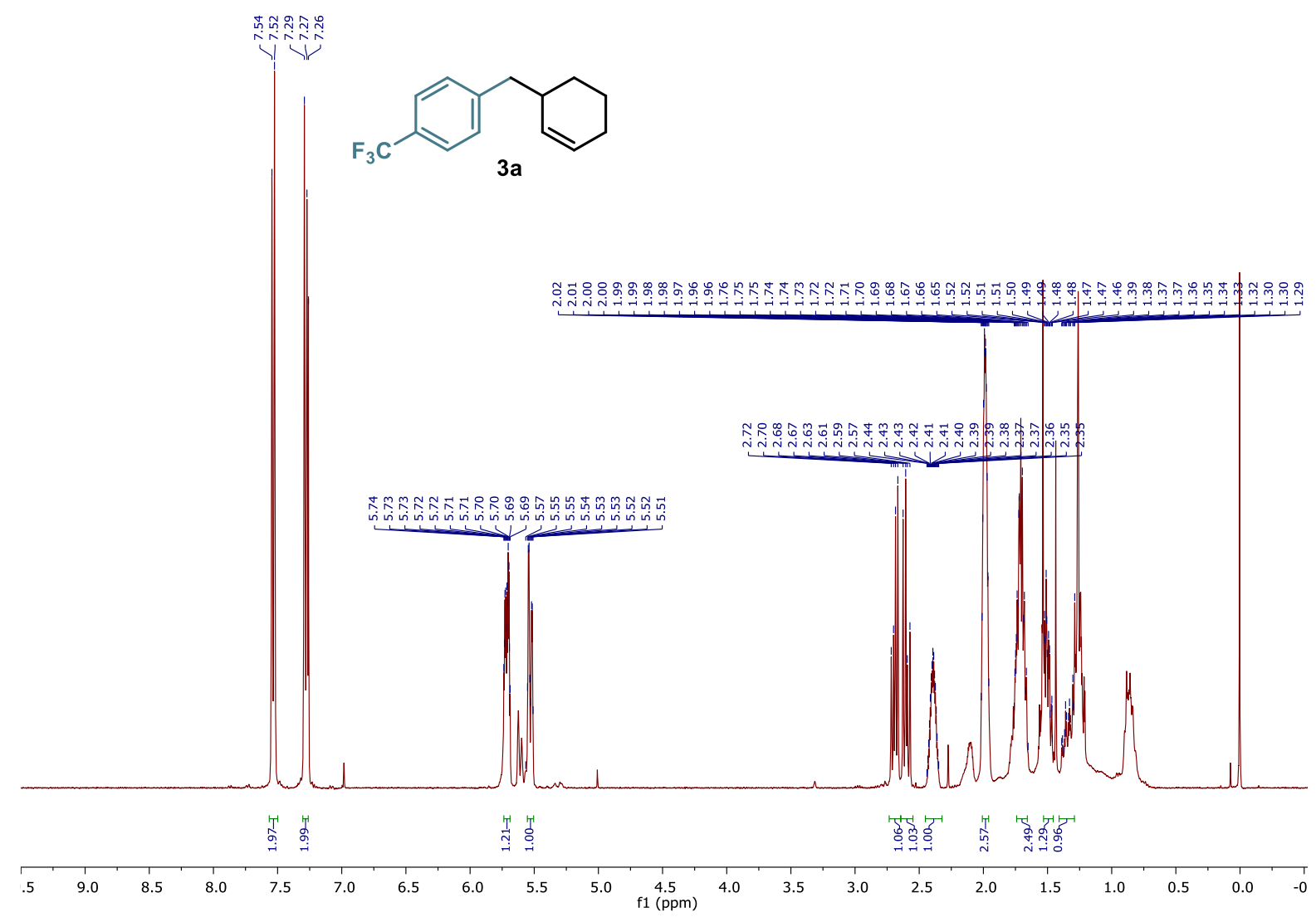

${ }^{13} \mathrm{C}$ NMR $\left(101 \mathrm{MHz}, \mathrm{CDCl}_{3}\right)$ of $\mathbf{3 a}$<smiles>FC(F)(F)c1ccc(CC2C=CCCC2)cc1</smiles>

3a

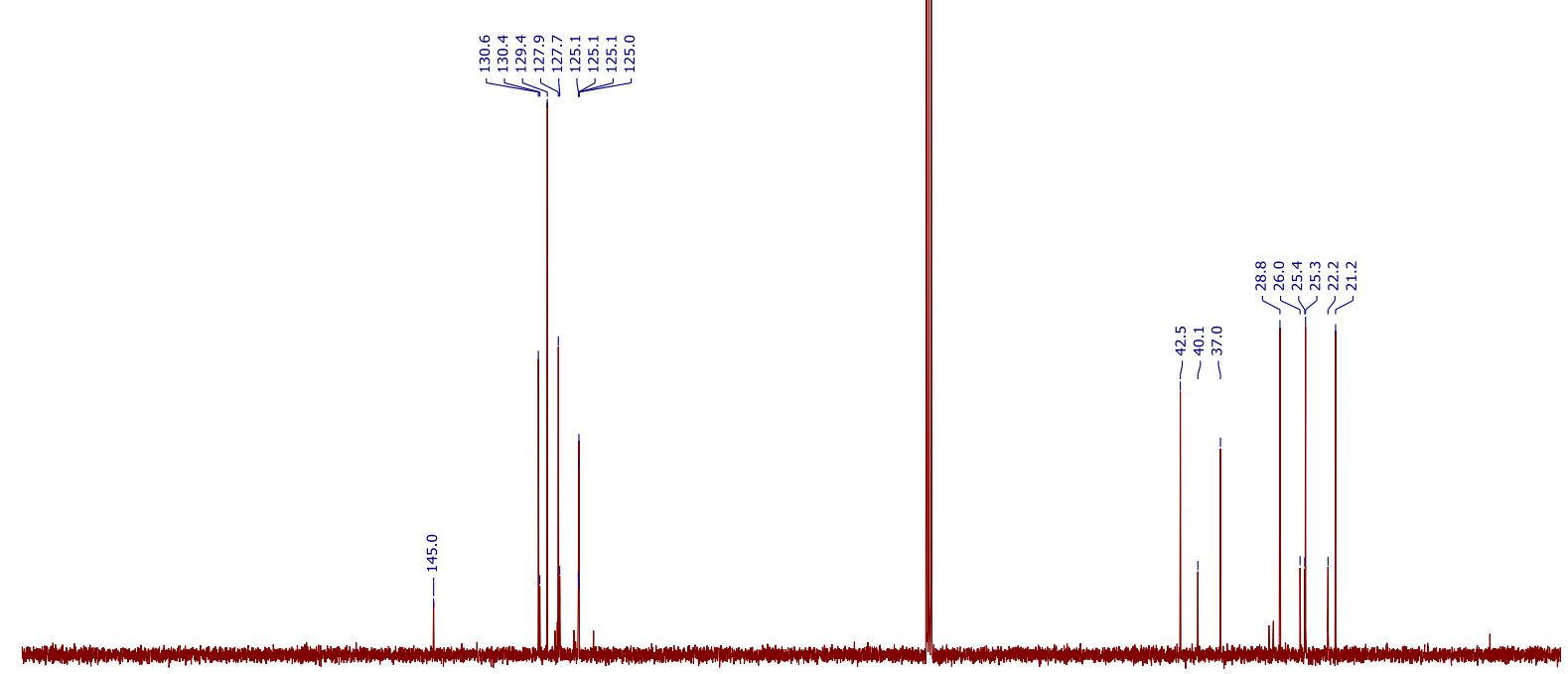

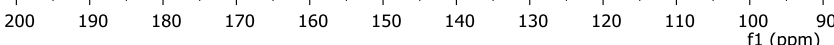


${ }^{19} \mathrm{~F}$ NMR (376 MHz, $\mathrm{CDCl}_{3}$ ) of $\mathbf{3 a}$

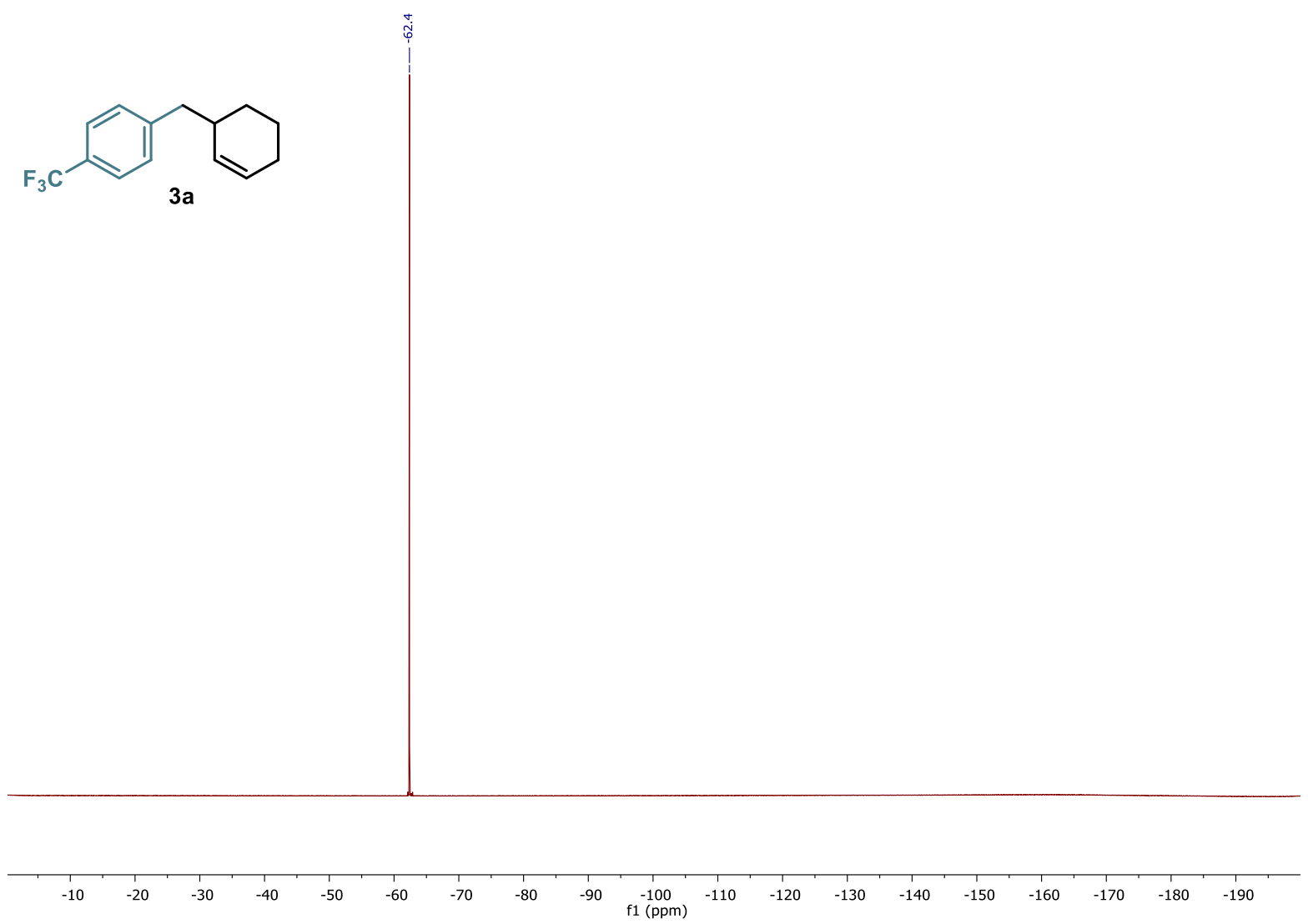


${ }^{1} \mathrm{H}$ NMR $\left(500 \mathrm{MHz}, \mathrm{CDCl}_{3}\right)$ of $\mathbf{3 b}$<smiles>N=C(N)C(c1ccccc1)C1C=CCCC1</smiles>

3b

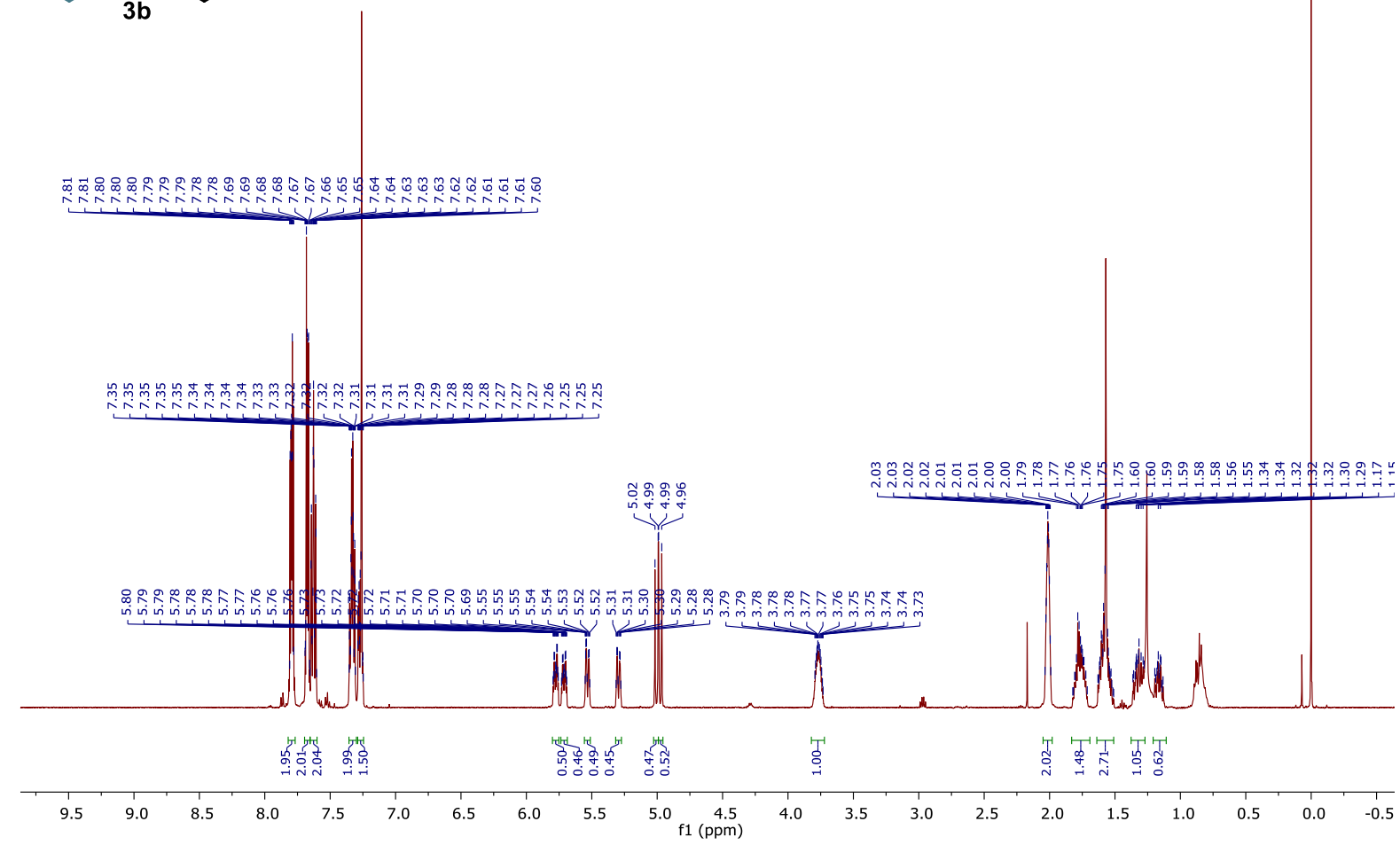

$\left.{ }^{13} \mathrm{C} \mathrm{NMR} \mathrm{(126} \mathrm{MHz,} \mathrm{CDCl}_{3}\right)$ of $\mathbf{3 b}$<smiles>[NH2+]C(c1ccccc1)C1C=CCCC1</smiles>

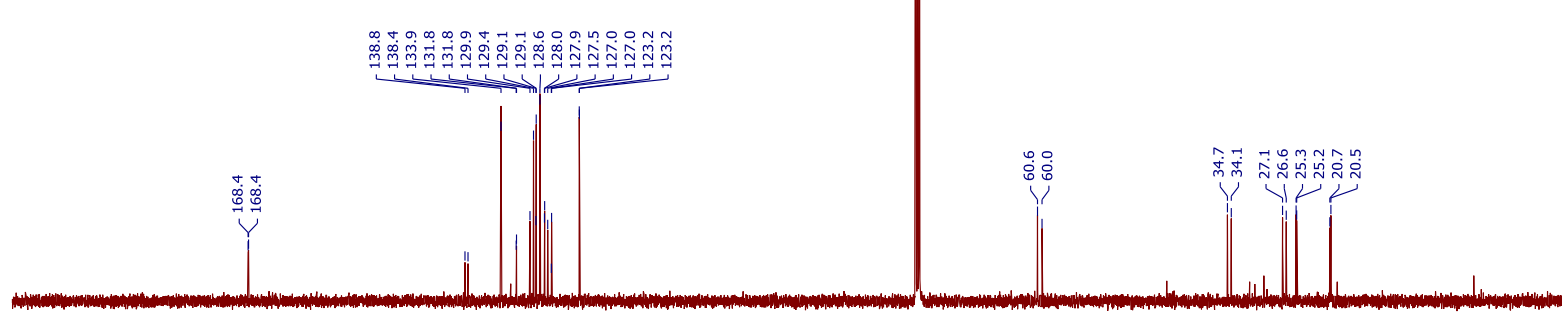

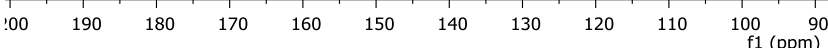


${ }^{1} \mathrm{H}$ NMR (400 MHz, $\mathrm{CDCl}_{3}$ ) of $\mathbf{3 c}$

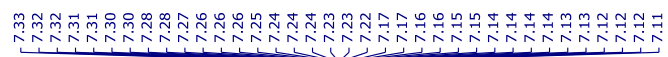<smiles>C1=CC(C(c2ccccc2)c2ccccc2)CCC1</smiles>

3c

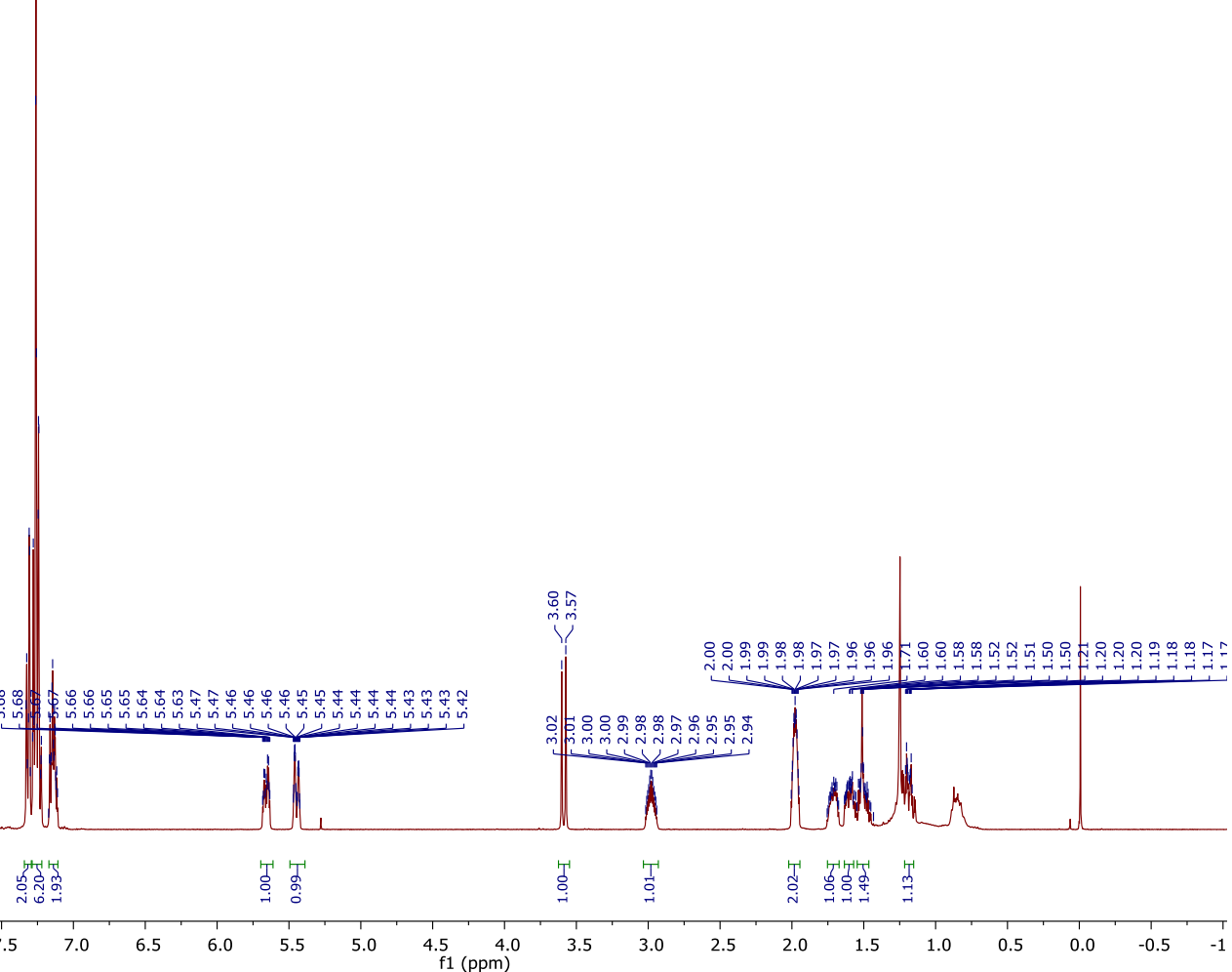

${ }^{13} \mathrm{C}$ NMR (101 MHz, $\left.\mathrm{CDCl}_{3}\right)$ of $\mathbf{3 c}$<smiles>C1=CC(C(c2ccccc2)c2ccccc2)CCC1</smiles>

$3 c$

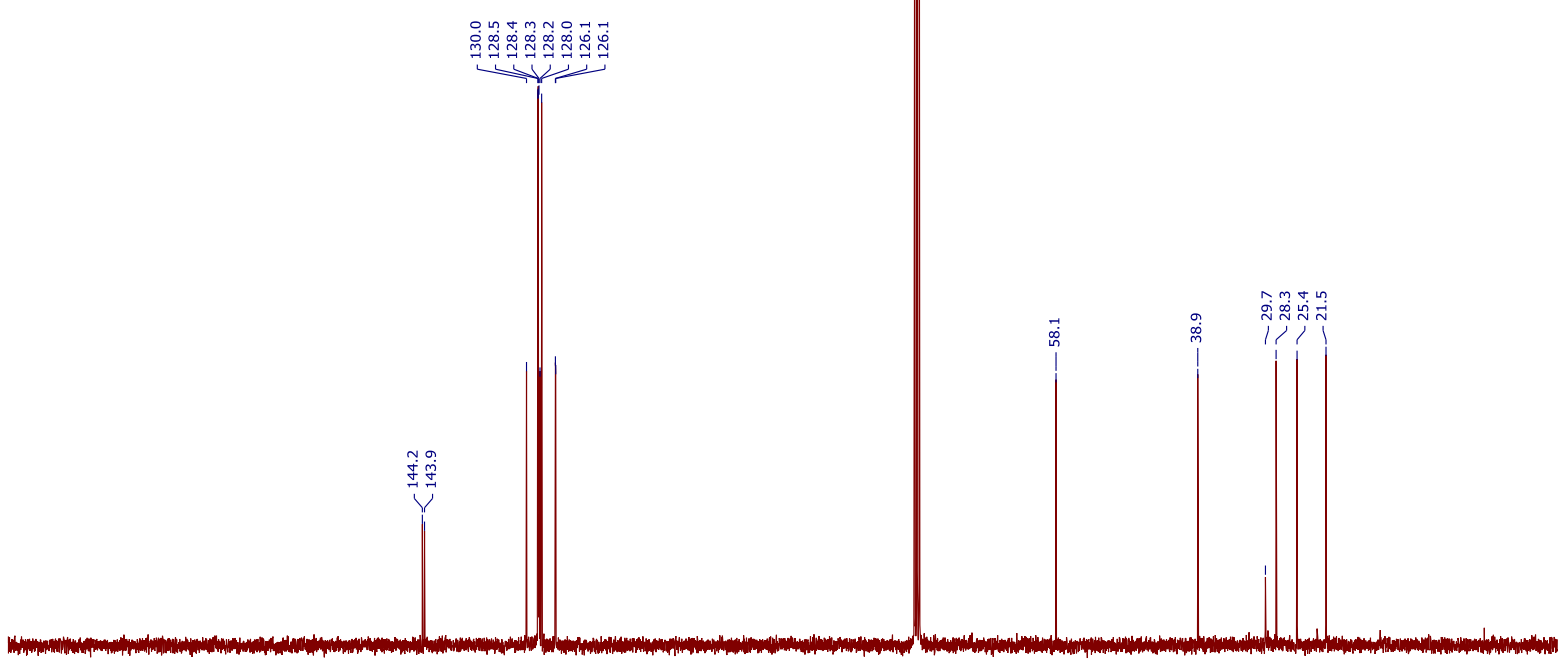

$\begin{array}{lllllllllll}00 & 190 & 180 & 170 & 160 & 150 & 140 & 130 & 120 & 110 & 100 \\ f 1(\mathrm{ppm}) & 90\end{array}$

80

50

30 
${ }^{1} \mathrm{H}$ NMR $\left(500 \mathrm{MHz}, \mathrm{CDCl}_{3}\right)$ of $\mathbf{3 d}$<smiles>CC(C)(c1ccccc1)C1C=CCCC1</smiles>

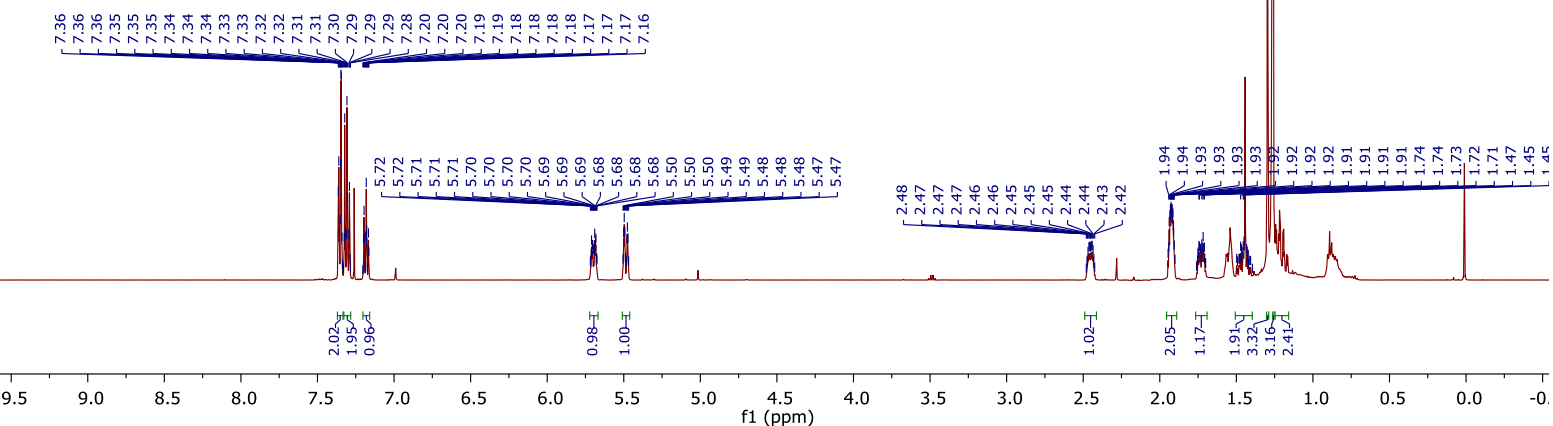

${ }^{13} \mathrm{C}$ NMR $\left(126 \mathrm{MHz}, \mathrm{CDCl}_{3}\right)$ of $\mathbf{3 d}$<smiles>CC(C)(c1ccccc1)C1C=CCCC1</smiles>

3d

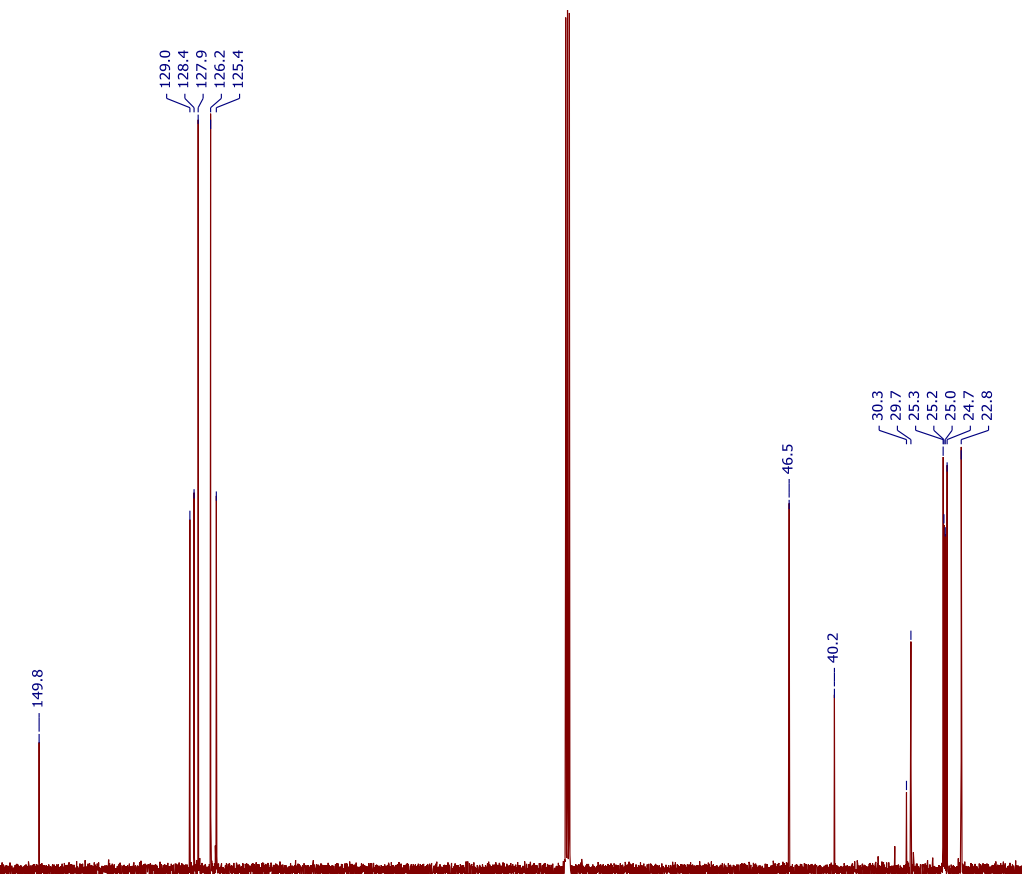

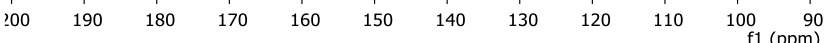
80 $60 \quad 50$ 20 
${ }^{1} \mathrm{H}$ NMR $\left(400 \mathrm{MHz}, \mathrm{CDCl}_{3}\right)$ of $\mathbf{3 e}$

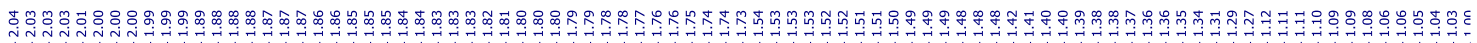<smiles>CC(C)(C)C1=CC(C(C)(C)c2ccccc2)CCC1</smiles>
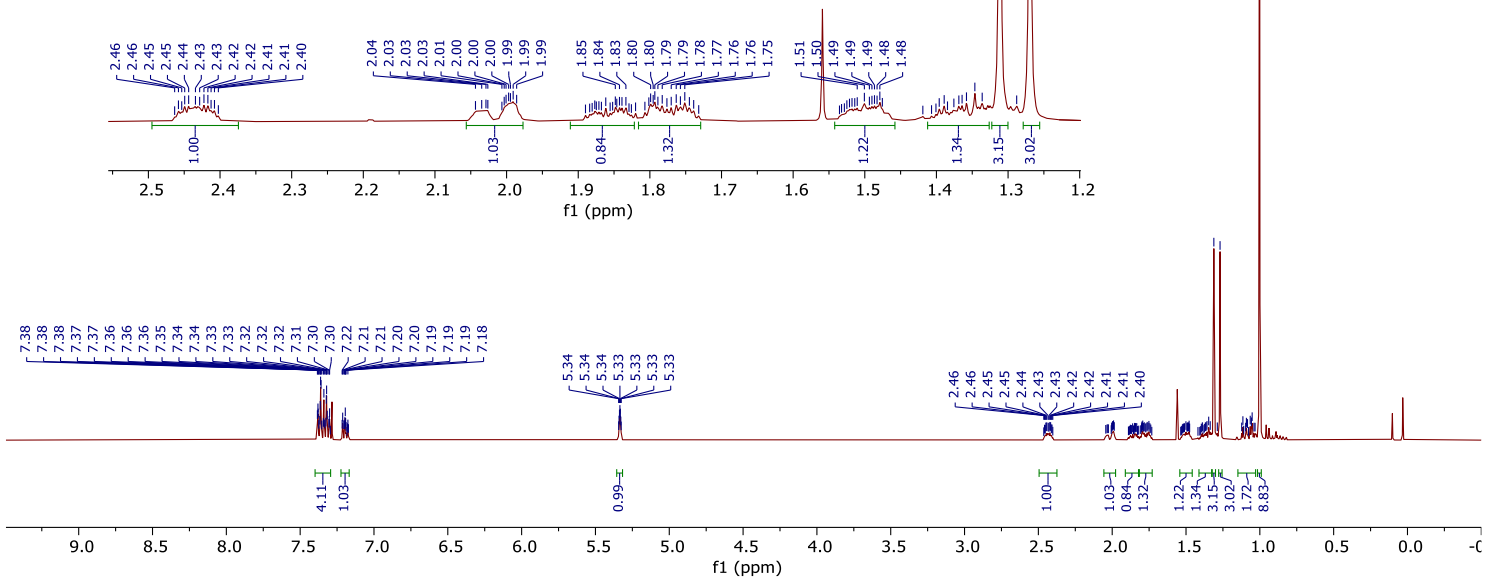

${ }^{13} \mathrm{C}$ NMR $\left(101 \mathrm{MHz}, \mathrm{CDCl}_{3}\right)$ of $\mathbf{3 e}$<smiles>CC(C)(C)C1=CC(C(C)(C)c2ccccc2)CCC1</smiles>

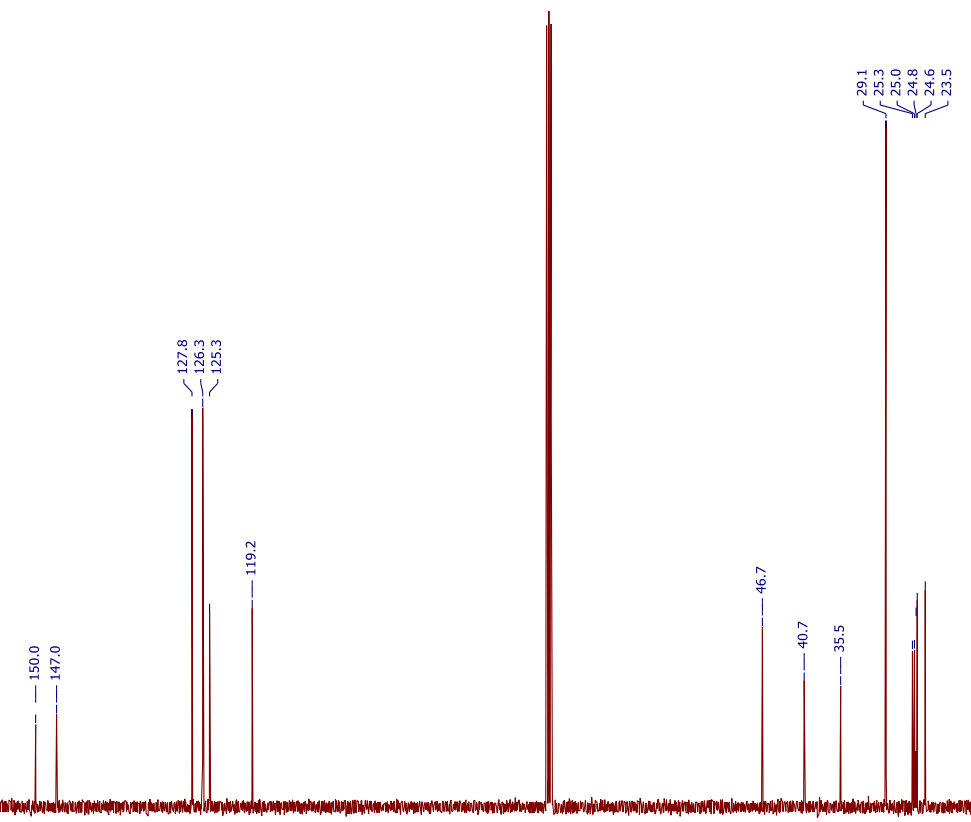

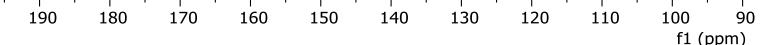


${ }^{1} \mathrm{H}$ NMR $\left(500 \mathrm{MHz}, \mathrm{CDCl}_{3}\right)$ of $\mathbf{3 f}$<smiles>COc1ccc2c(c1)c(CC1C=CCCC1)c(C)n2C(=O)c1ccc(Cl)cc1</smiles>

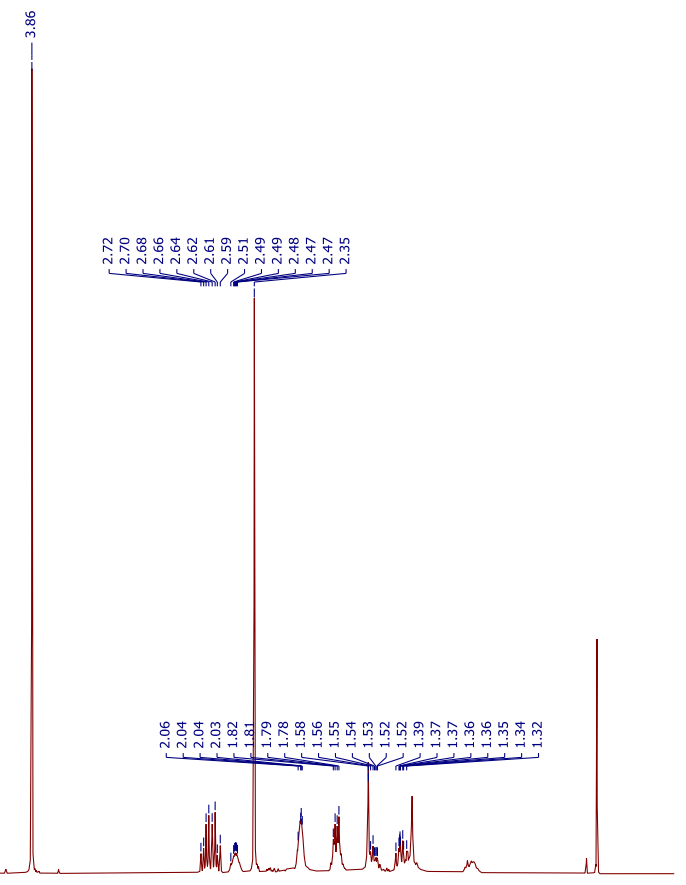

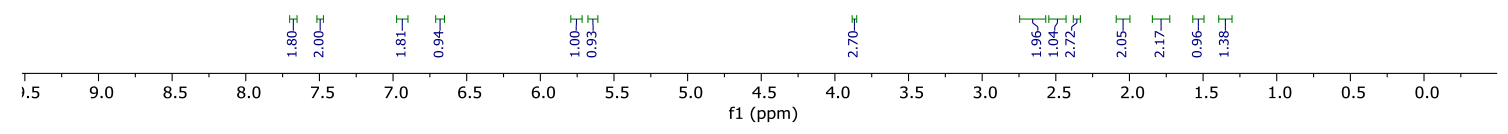

${ }^{13} \mathrm{C}$ NMR $\left(126 \mathrm{MHz}, \mathrm{CDCl}_{3}\right)$ of $\mathbf{3 f}$<smiles>COc1ccc2c(c1)c(CC1C=CCCC1)c(C)n2C(=O)c1ccc(Cl)cc1</smiles>
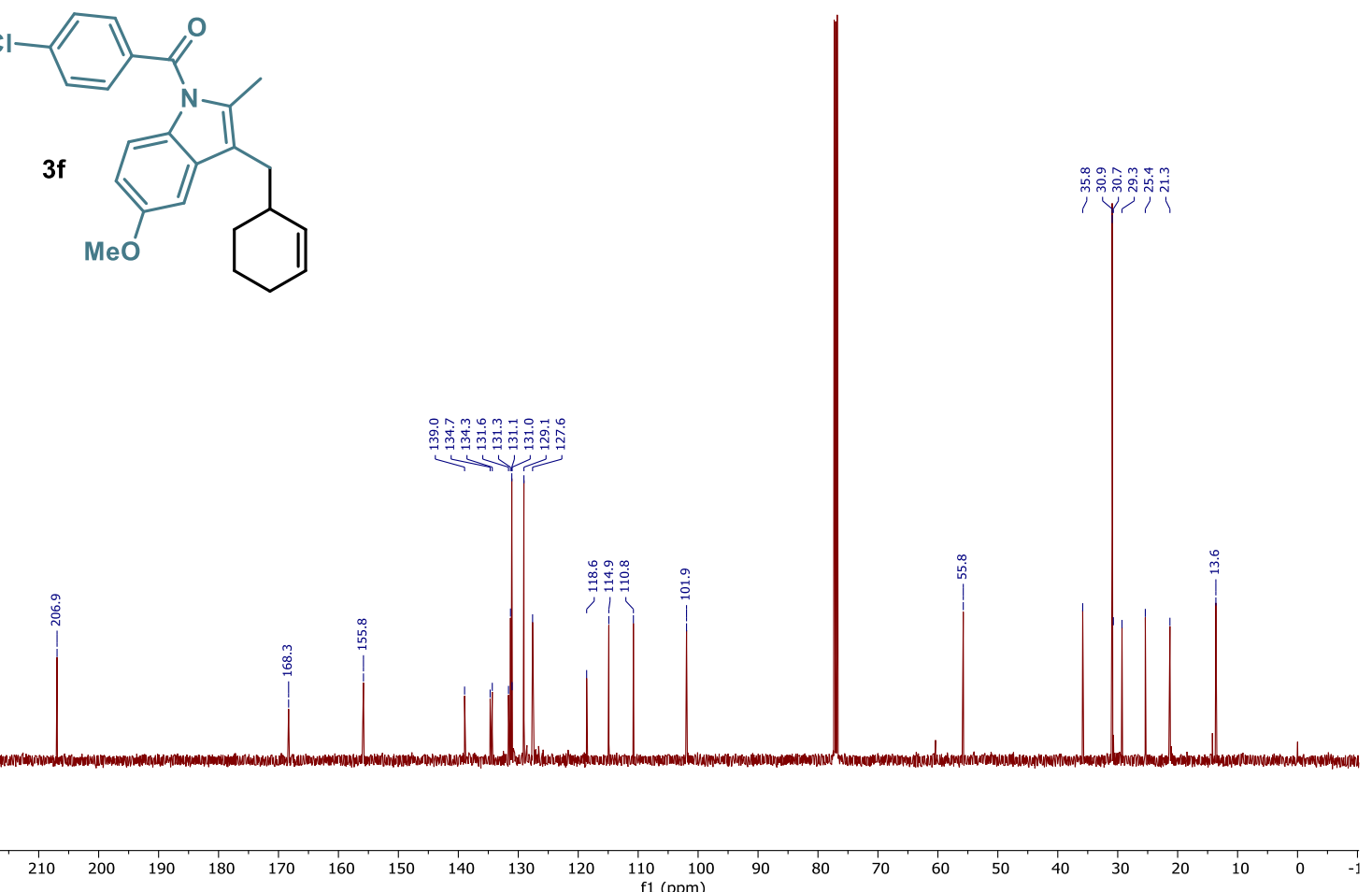
${ }^{1} \mathrm{H} \mathrm{NMR}\left(400 \mathrm{MHz}, \mathrm{CDCl}_{3}\right)$ of $\mathbf{3 g}$

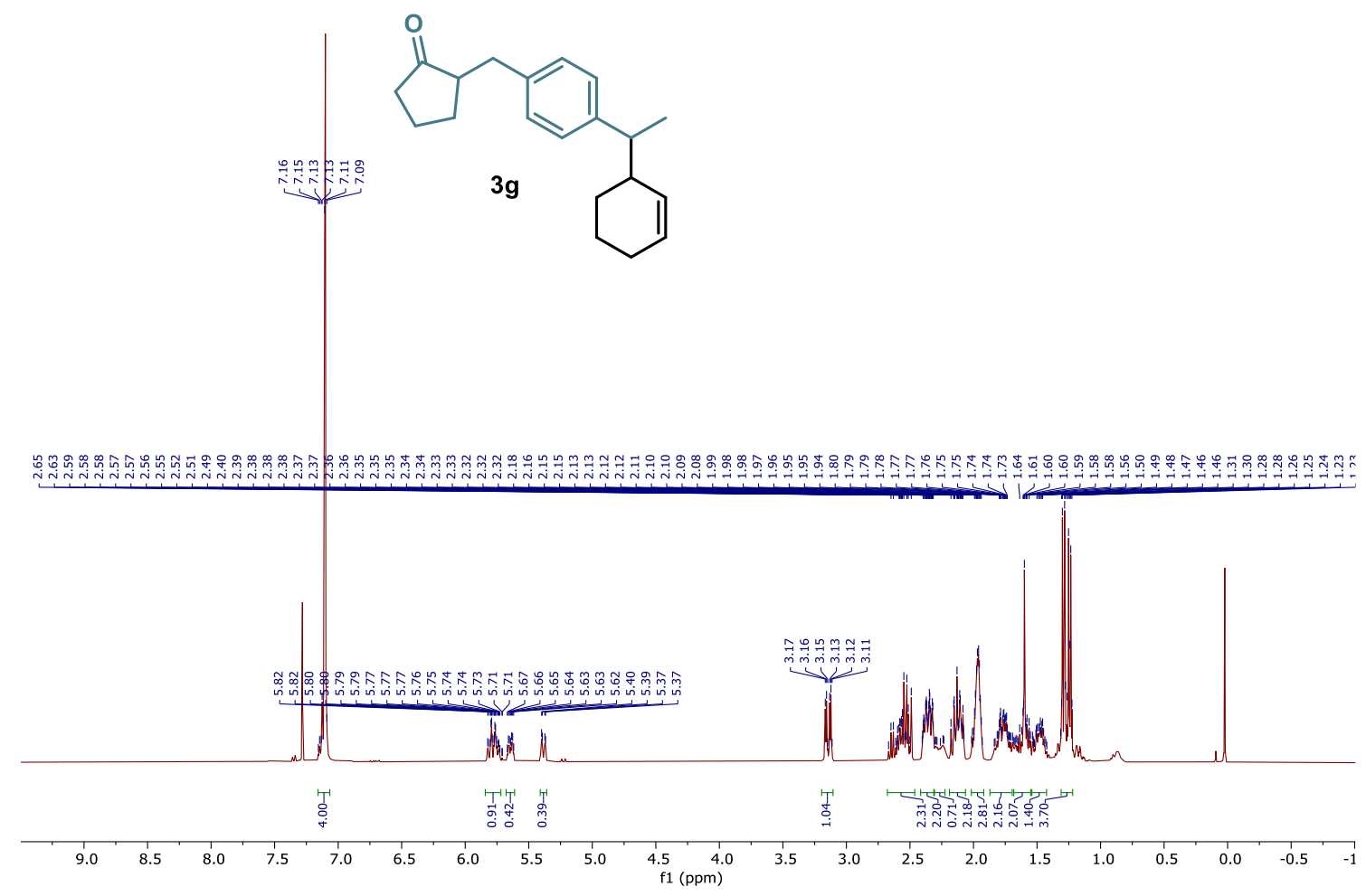

${ }^{13} \mathrm{C} \mathrm{NMR}\left(101 \mathrm{MHz}, \mathrm{CDCl}_{3}\right)$ of $\mathbf{3 g}$<smiles>CC(c1ccc(CC2CCCC2=O)cc1)C1C=CCCC1</smiles>

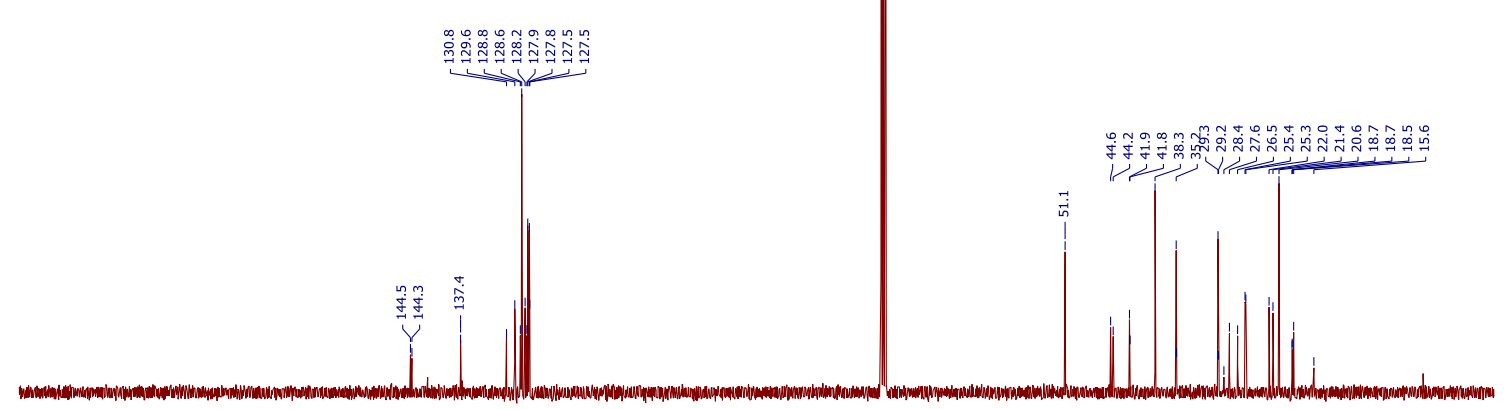

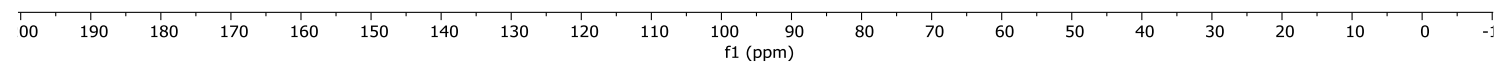


${ }^{1} \mathrm{H}$ NMR (400 MHz, $\mathrm{CDCl}_{3}$ ) of $\mathbf{3 h}$

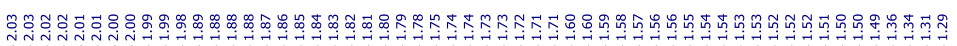

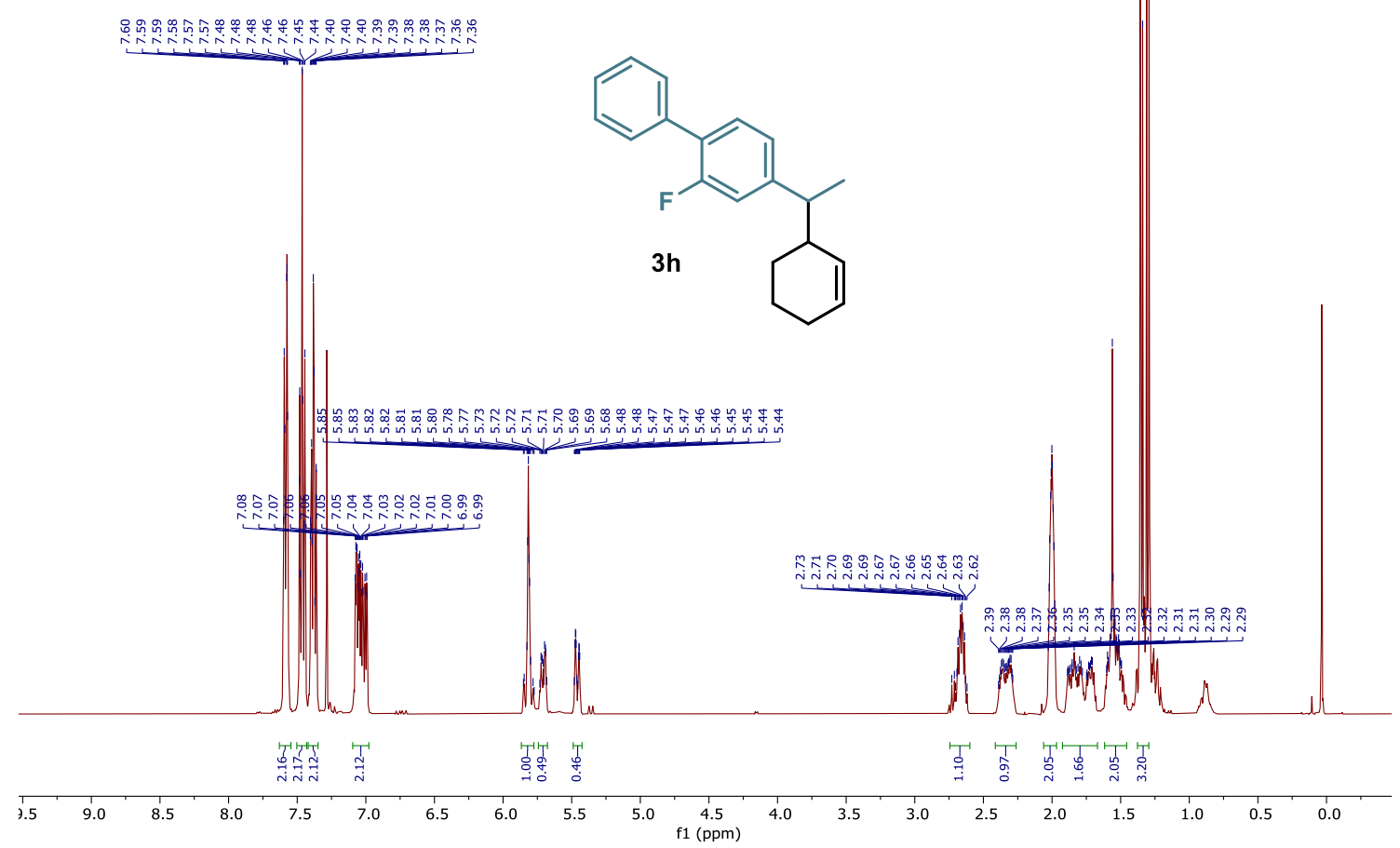

${ }^{13} \mathrm{C}$ NMR (101 MHz, $\left.\mathrm{CDCl}_{3}\right)$ of $\mathbf{3 h}$
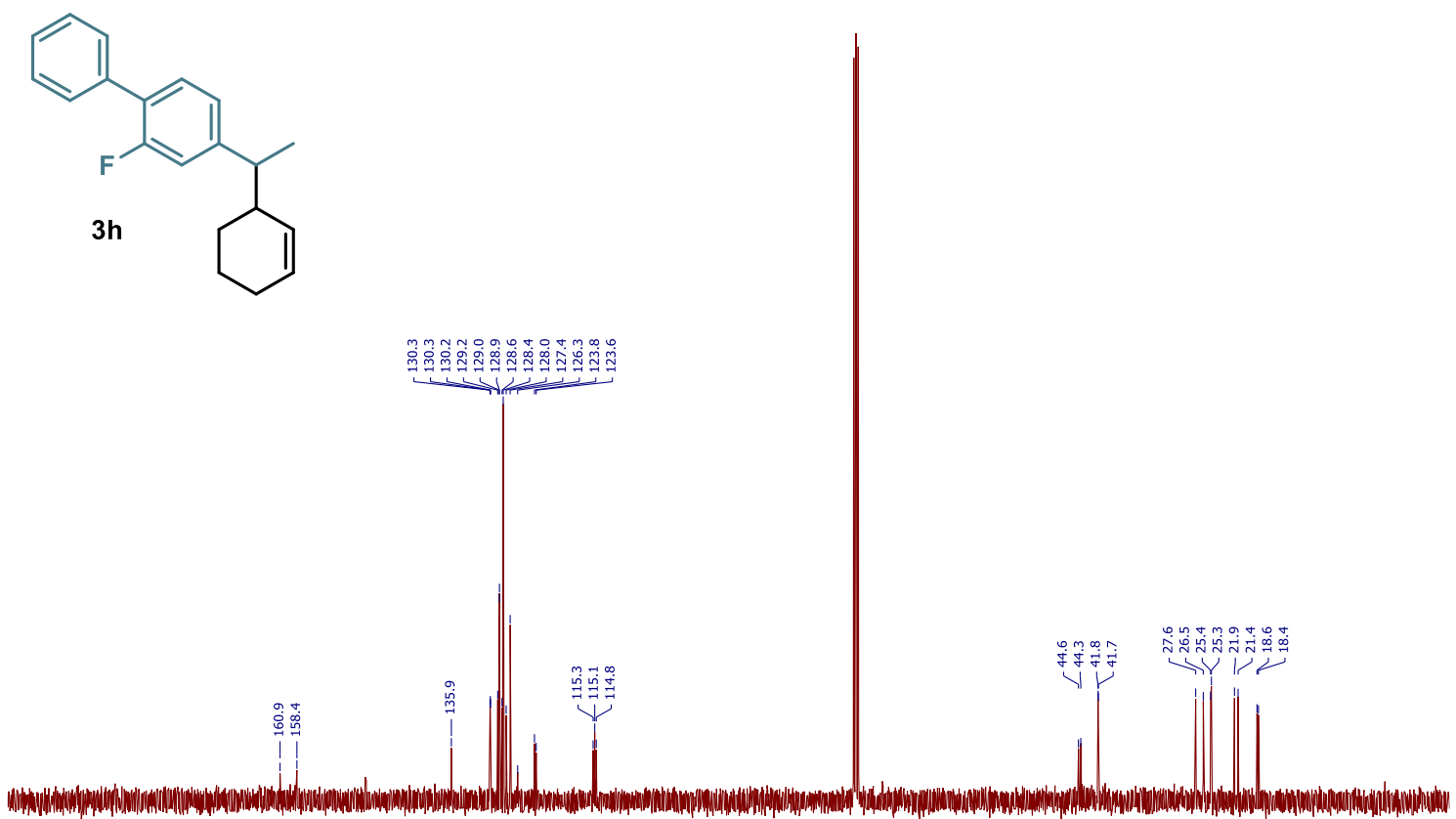

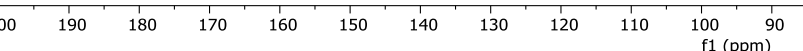


${ }^{19} \mathrm{~F}$ NMR (376 MHz, $\mathrm{CDCl}_{3}$ ) of $\mathbf{3 h}$

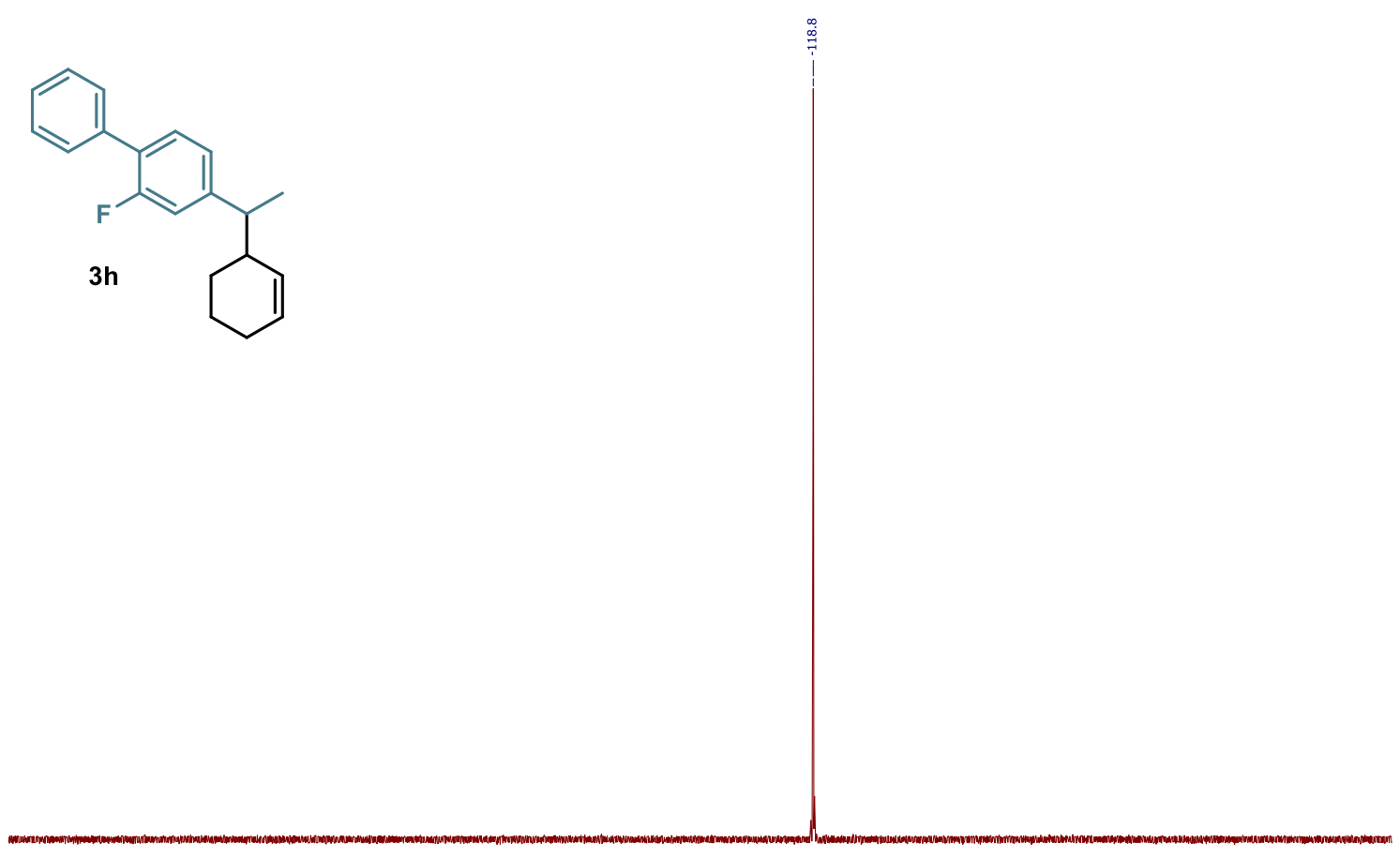

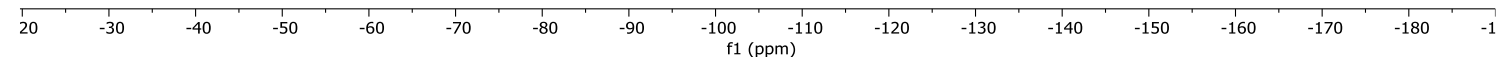


${ }^{1} \mathrm{H}$ NMR $\left(500 \mathrm{MHz}, \mathrm{CDCl}_{3}\right.$ ) of $\mathbf{3 i}$

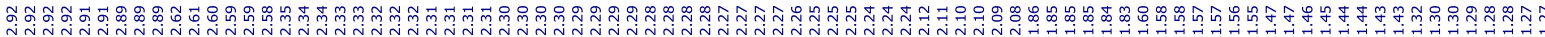

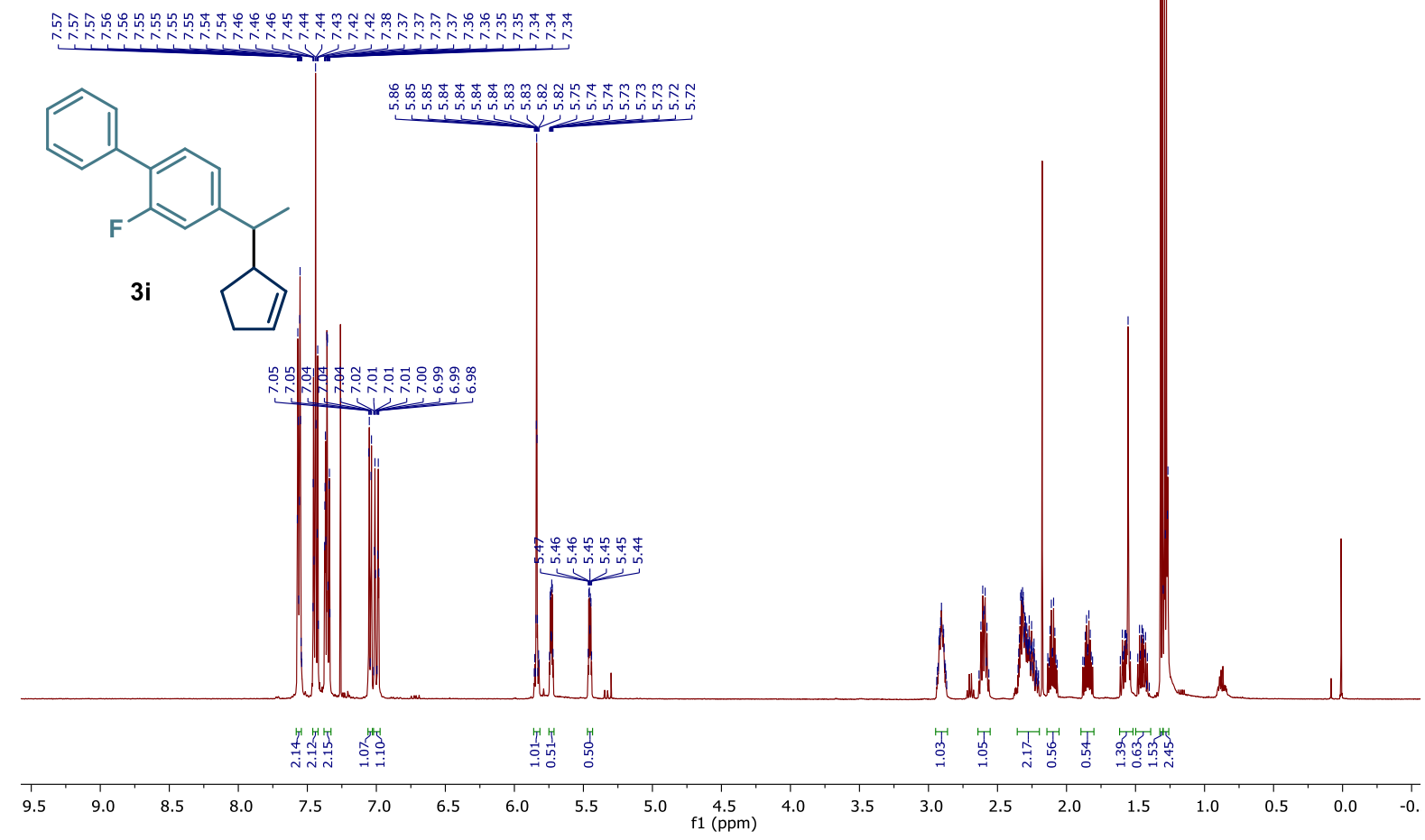

${ }^{13} \mathrm{C}$ NMR $\left(126 \mathrm{MHz}, \mathrm{CDCl}_{3}\right)$ of $\mathbf{3 i}$

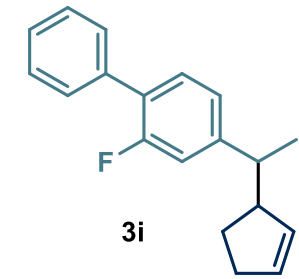

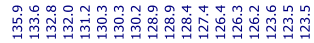

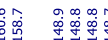

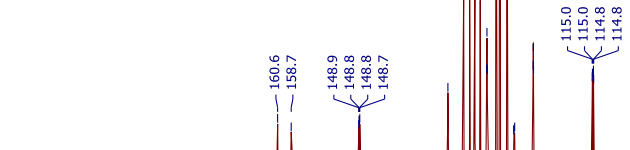

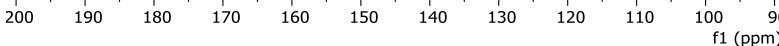
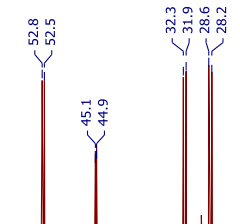
${ }^{19} \mathrm{~F}$ NMR $\left(376 \mathrm{MHz}, \mathrm{CDCl}_{3}\right)$ of $\mathbf{3 i}$

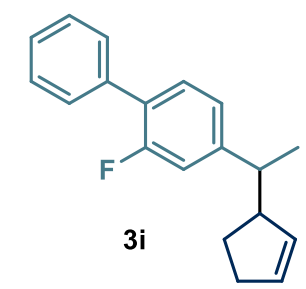

$\stackrel{\infty}{\sharp}$

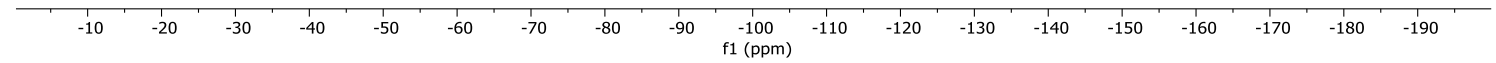


${ }^{1} \mathrm{H}$ NMR (400 MHz, $\left.\mathrm{CDCl}_{3}\right)$ of $\mathbf{3 j}$

gong<smiles>CC(c1ccc(-c2ccccc2)c(F)c1)C1C=C(C(C)(C)C)CCC1</smiles>

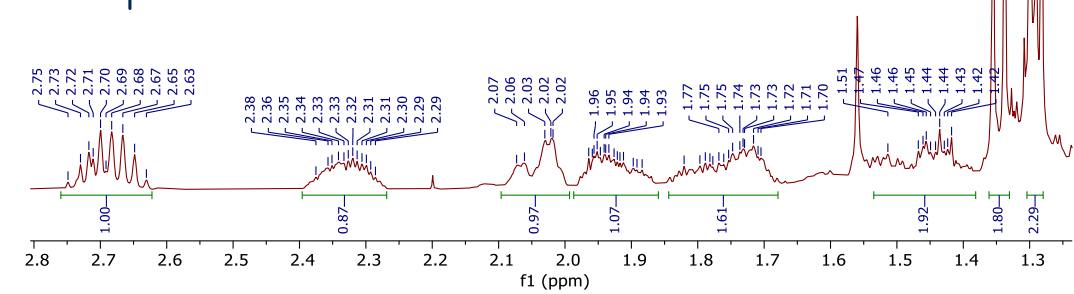

管
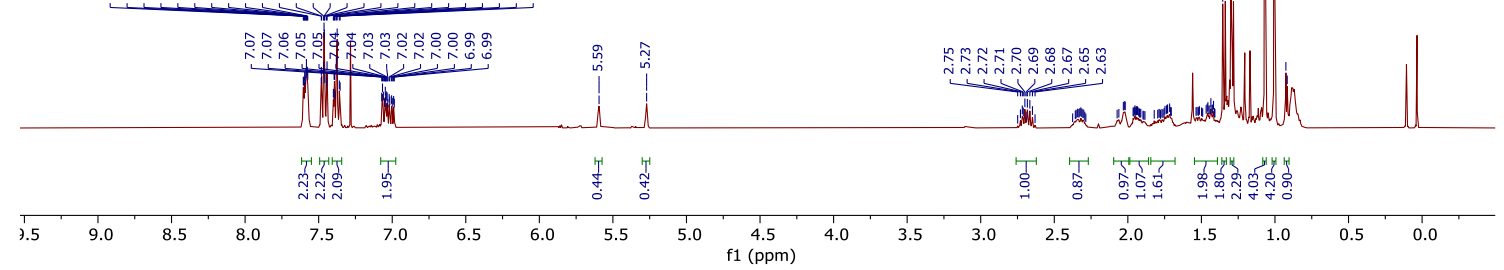

${ }^{13} \mathrm{C}$ NMR $\left(101 \mathrm{MHz}, \mathrm{CDCl}_{3}\right)$ of $\mathbf{3 j}$<smiles>CC(c1ccc(-c2ccccc2)c(F)c1)C1C=C(C(C)(C)C)CCC1</smiles>

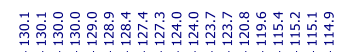

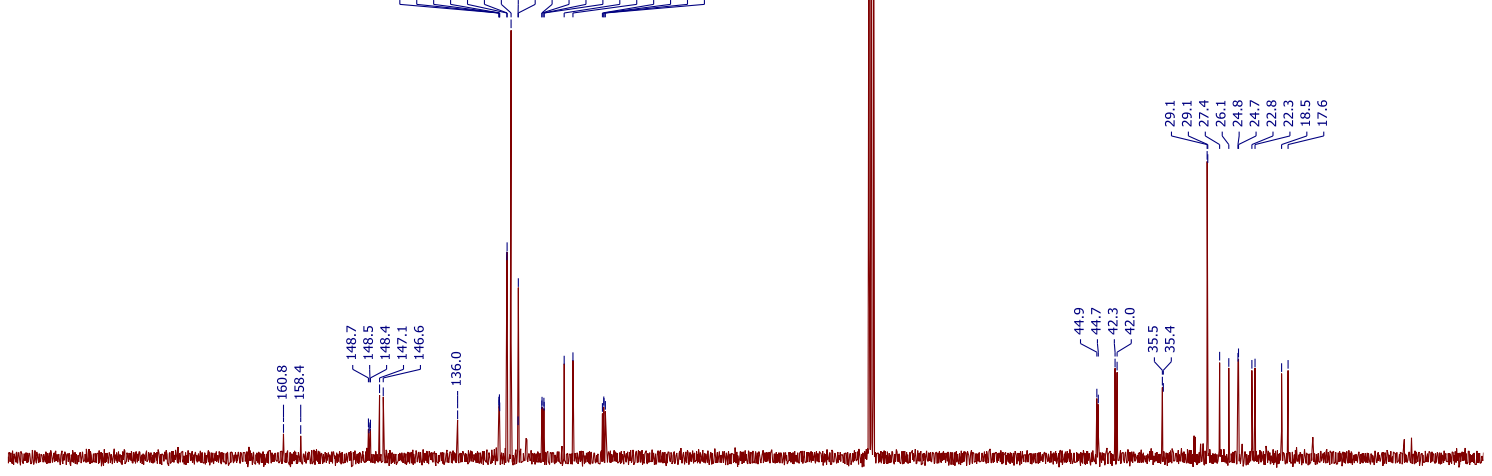

$00 \quad 190$
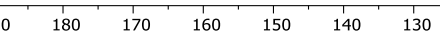

$100 \quad 90$
f1 (ppm) 
${ }^{19} \mathrm{~F}$ NMR $\left(376 \mathrm{MHz}, \mathrm{CDCl}_{3}\right)$ of $\mathbf{3 j}$

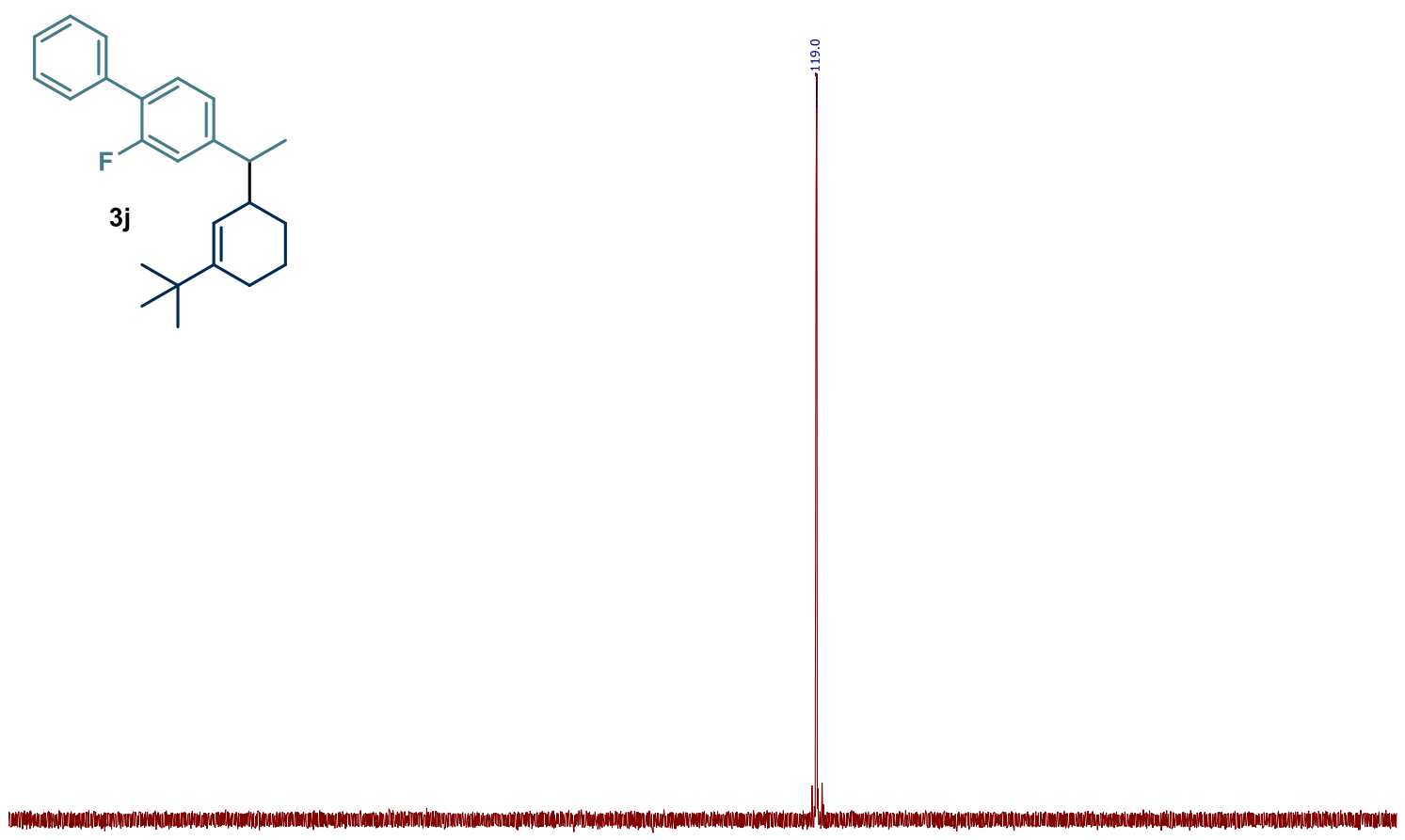

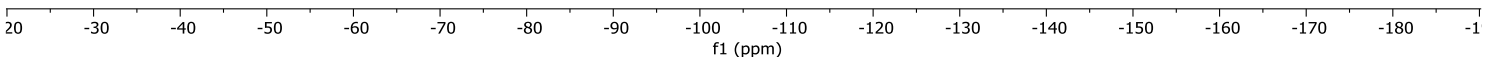


${ }^{1} \mathrm{H}$ NMR $\left(500 \mathrm{MHz}, \mathrm{CDCl}_{3}\right.$ ) of $\mathbf{3 k}$

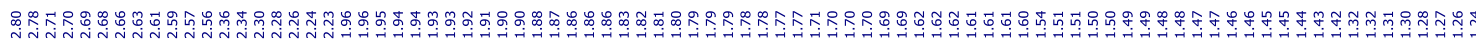

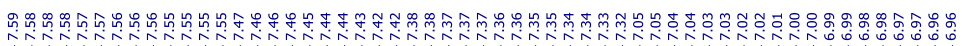
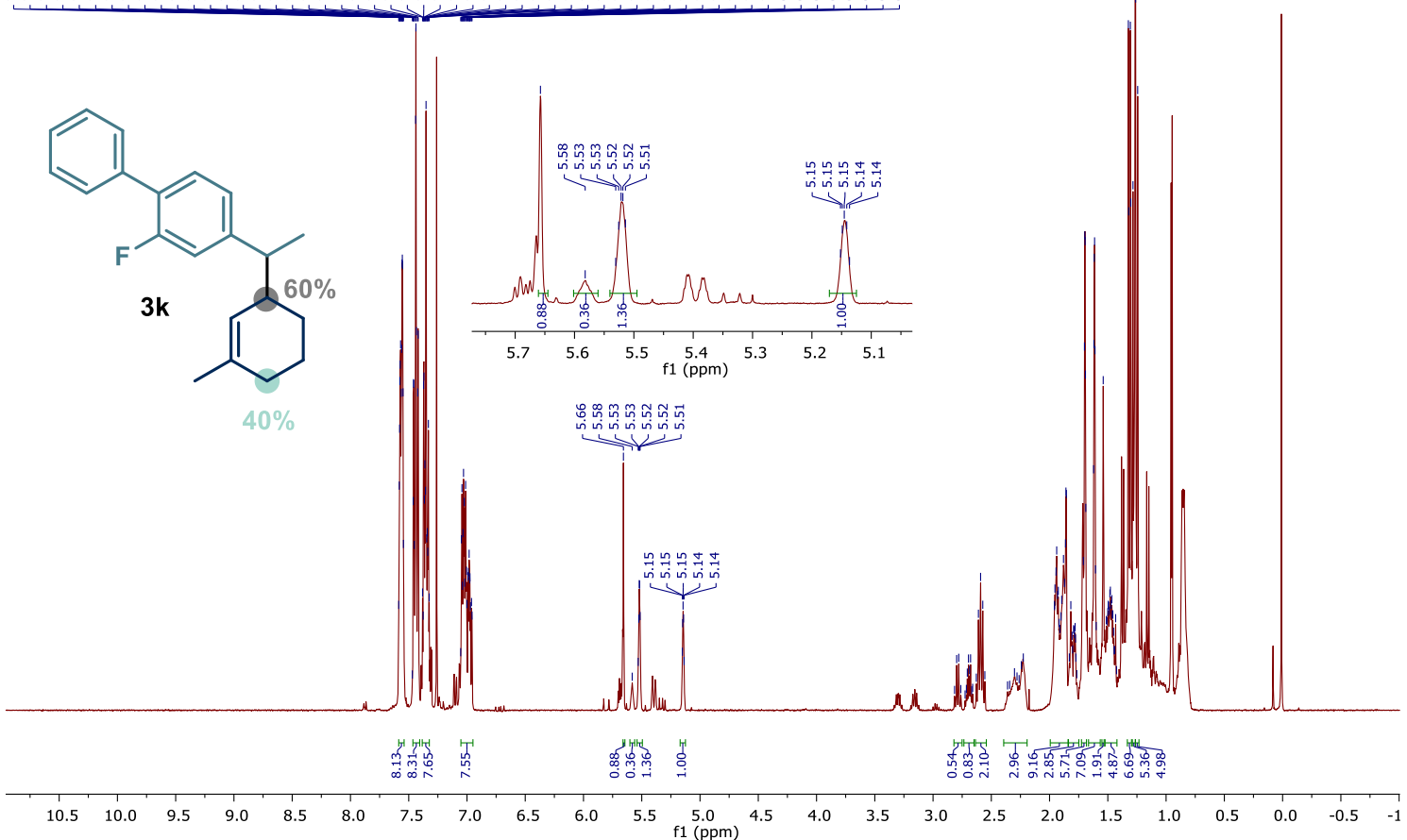

$\left.{ }^{13} \mathrm{C} \mathrm{NMR} \mathrm{(126} \mathrm{MHz,} \mathrm{CDCl}_{3}\right)$ of $\mathbf{3 k}$
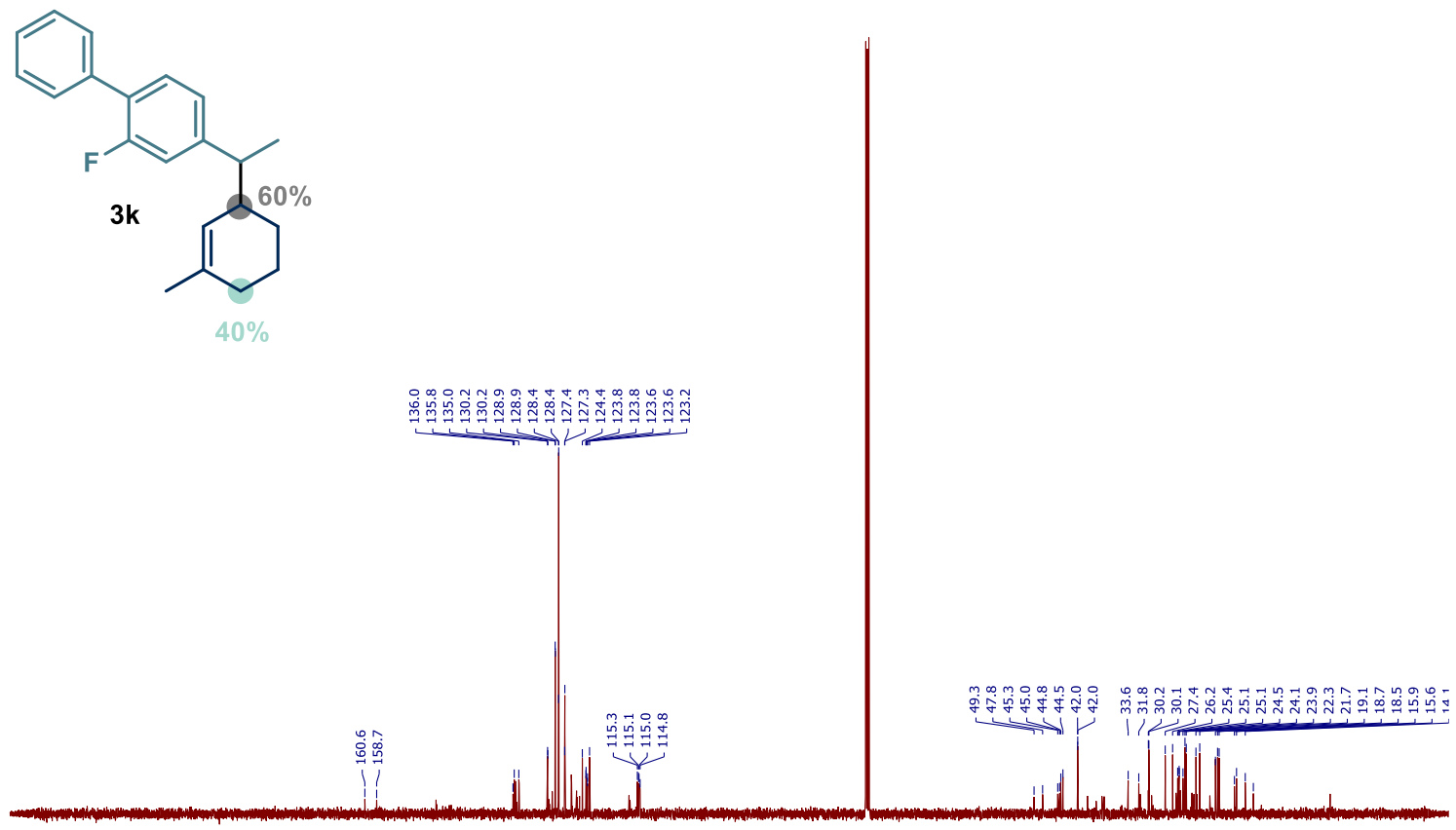

$\begin{array}{lllllllllllllllllllllllllllllllll}210 & 200 & 190 & 180 & 170 & 160 & 150 & 140 & 130 & 120 & 110 & 100 & 90 & 80 & 70 & 60 & 50 & 40 & 30 & 20 & 10 & 0 & -10\end{array}$ 
${ }^{19} \mathrm{~F}$ NMR (376 MHz, $\mathrm{CDCl}_{3}$ ) of $\mathbf{3 k}$

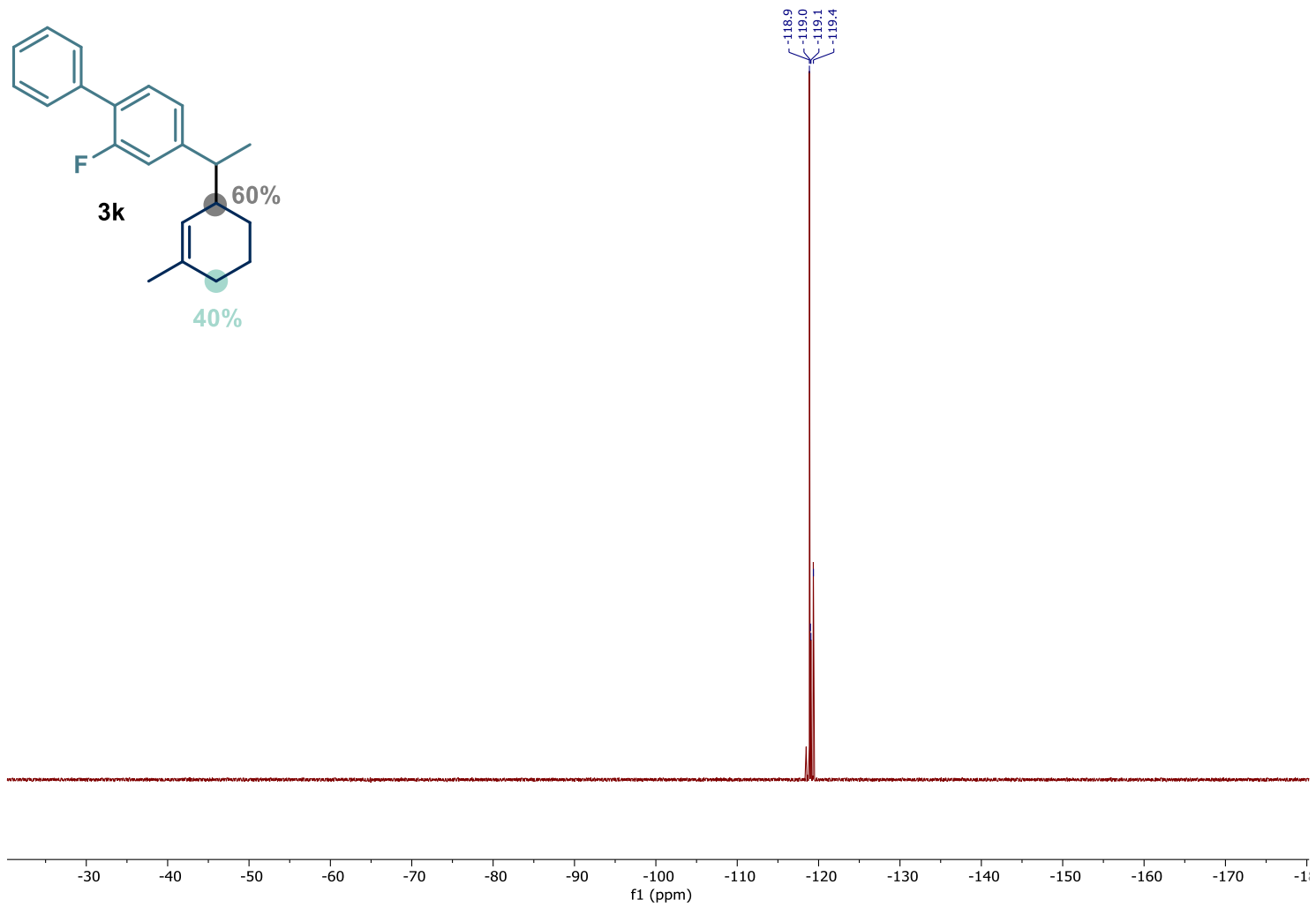


${ }^{1} \mathrm{H}$ NMR (500 MHz, $\left.\mathrm{CDCl}_{3}\right)$ of $\mathbf{3 I}$<smiles>CC(=O)C(c1ccccc1)C1C=CCCC1</smiles>

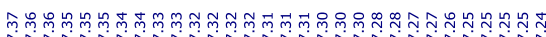

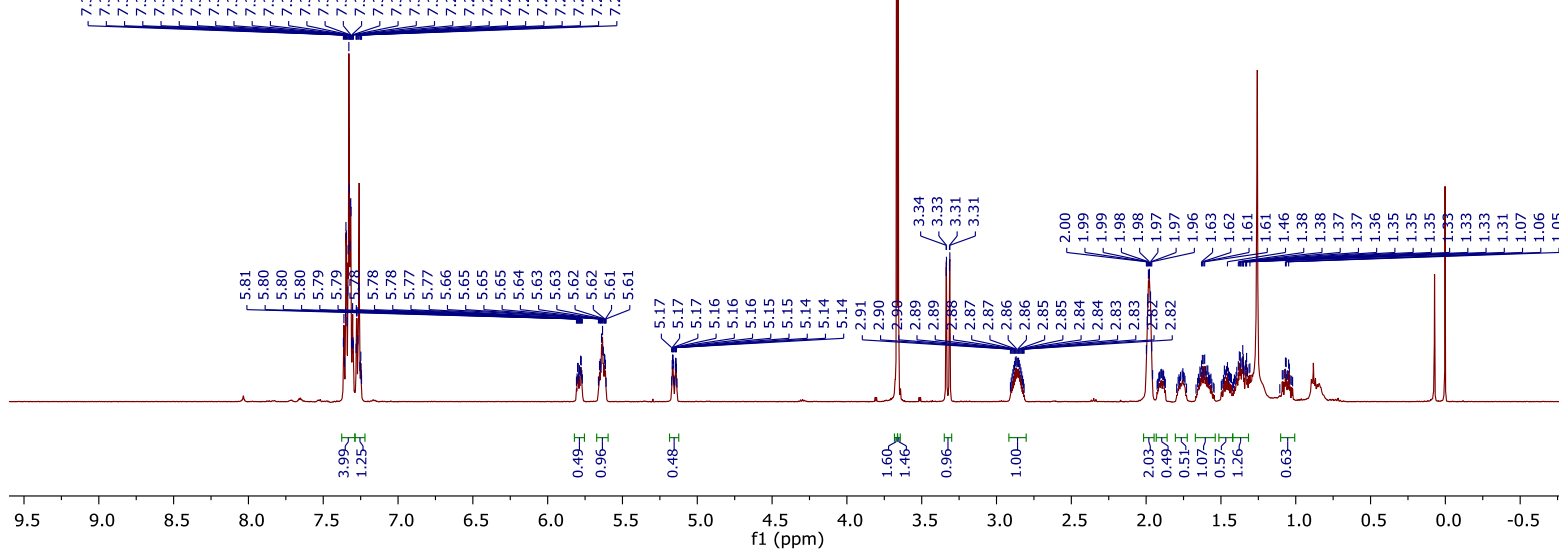

${ }^{13} \mathrm{C} \mathrm{NMR}\left(126 \mathrm{MHz}, \mathrm{CDCl}_{3}\right)$ of $\mathbf{3 l}$<smiles>CC(=O)C(c1ccccc1)C1C=CCCC1</smiles>

31
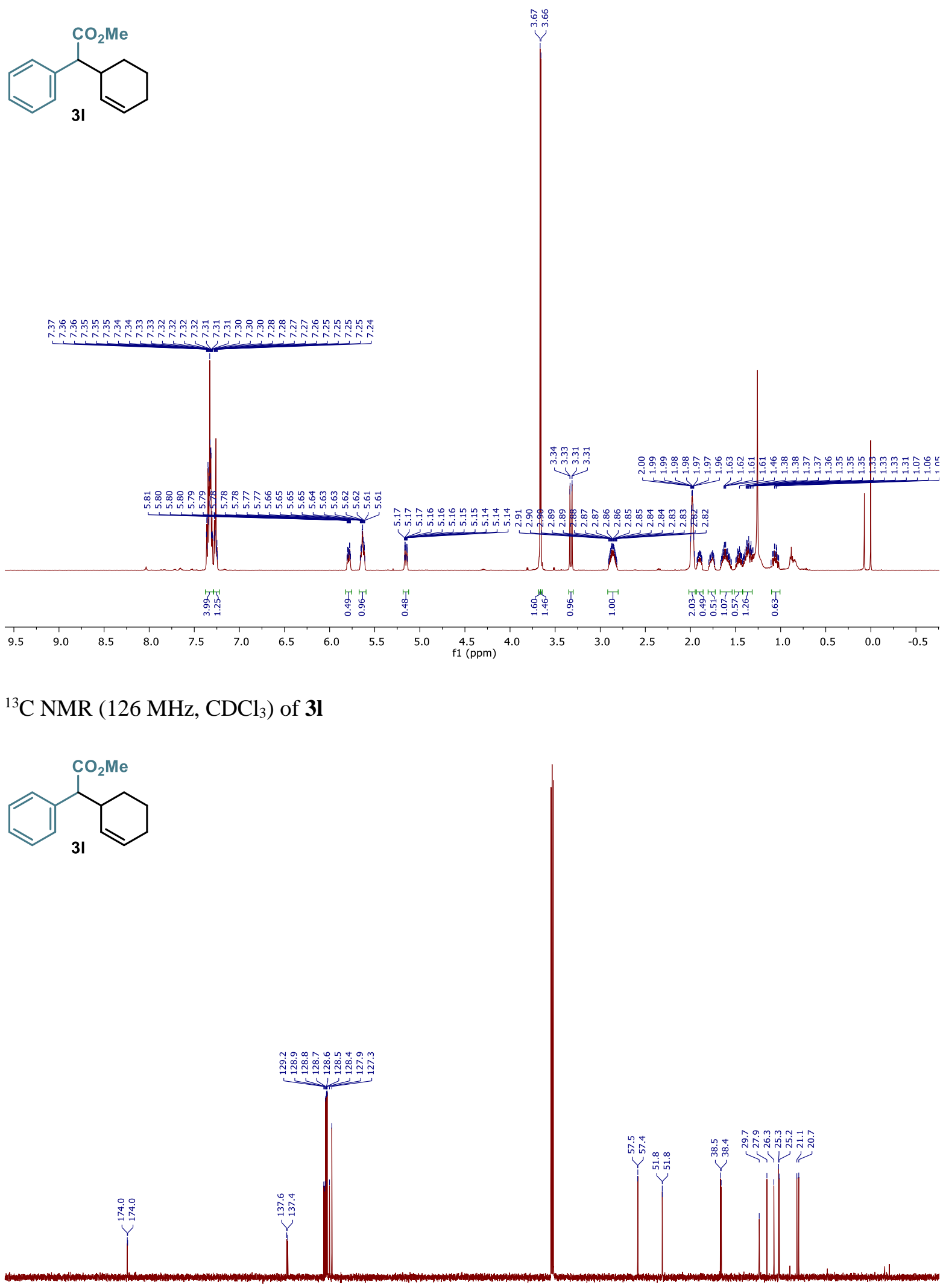

$\begin{array}{lllllllllll}200 & 190 & 180 & 170 & 160 & 150 & 140 & 130 & 120 & 110 & 100\end{array}$ $80 \quad 70$ 1. 40 20 
${ }^{1} \mathrm{H} \mathrm{NMR}\left(500 \mathrm{MHz}, \mathrm{CDCl}_{3}\right)$ of $\mathbf{6 a}$<smiles>CCOC(=O)CCC(c1ccccc1)(c1ccccc1)C1C=CCCC1</smiles>

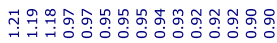

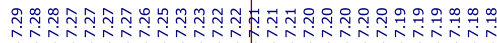

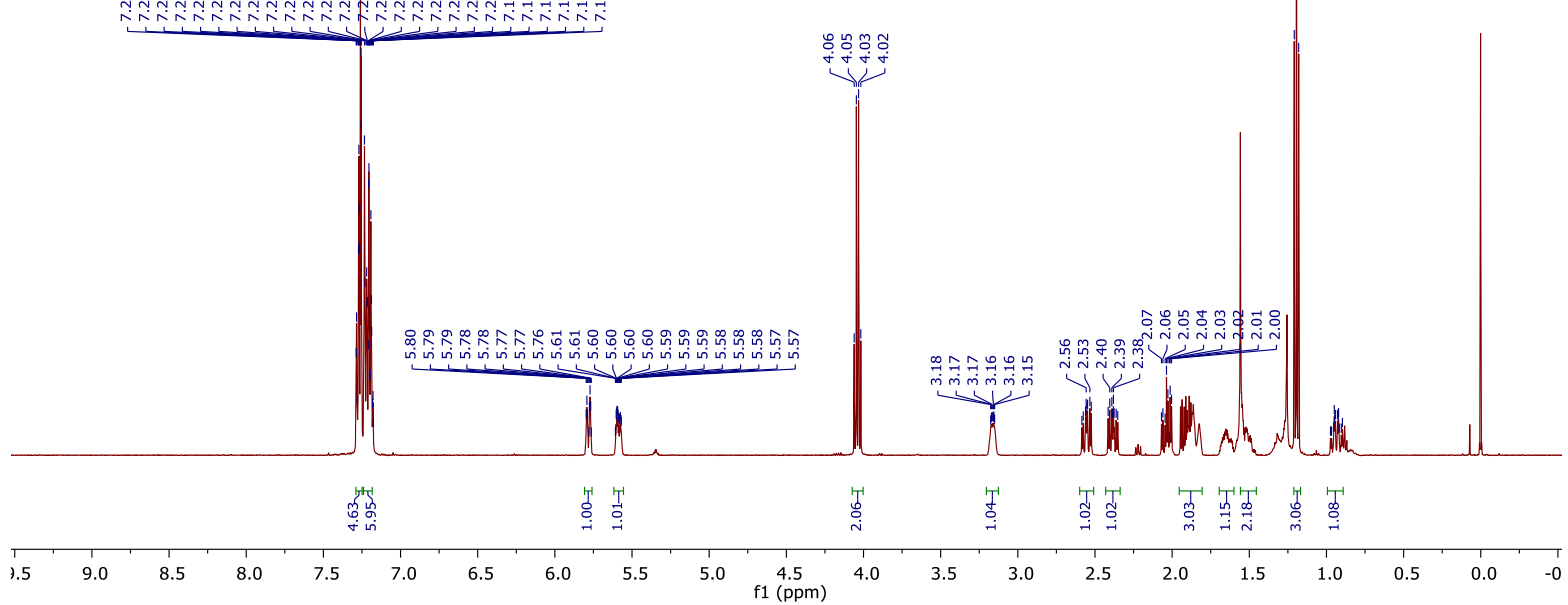

${ }^{13} \mathrm{C}$ NMR (126 MHz, $\left.\mathrm{CDCl}_{3}\right)$ of $\mathbf{6 a}$ 

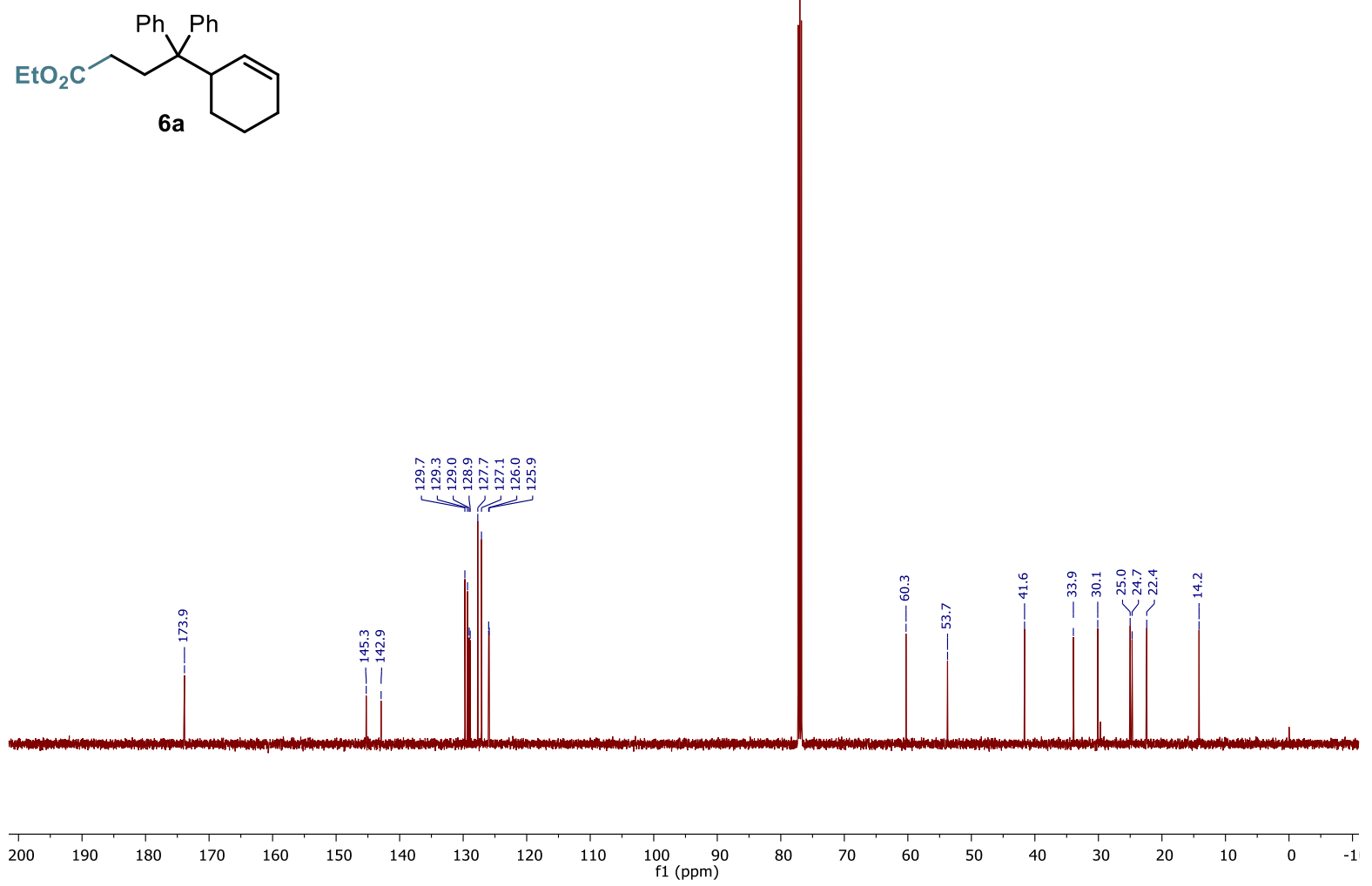

${ }^{1} \mathrm{H}$ NMR (400 MHz, $\left.\mathrm{CDCl}_{3}\right)$ of $\mathbf{6 b}$
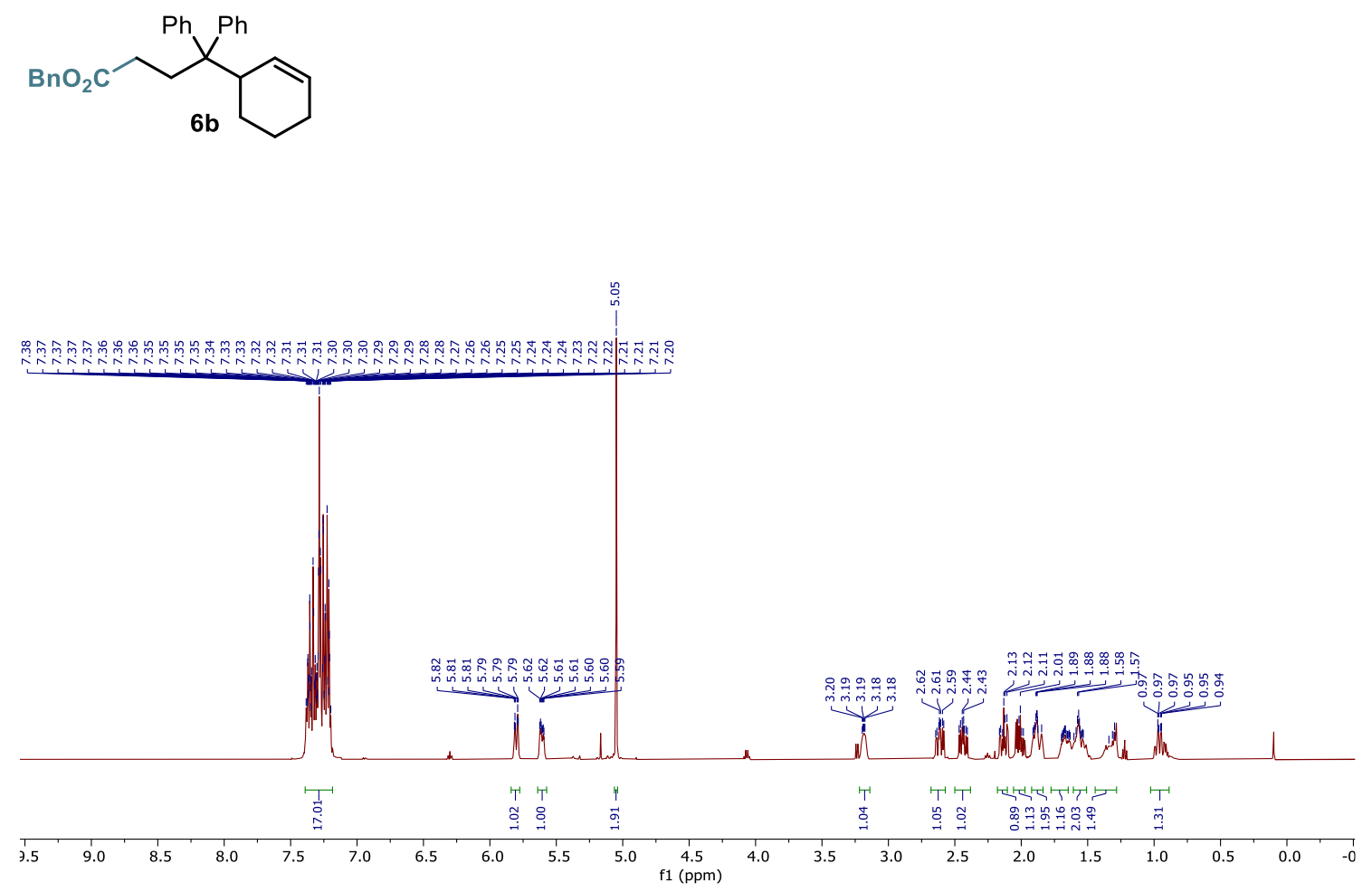

${ }^{13} \mathrm{C}$ NMR $\left(126 \mathrm{MHz}, \mathrm{CDCl}_{3}\right)$ of $\mathbf{6 b}$

S73 

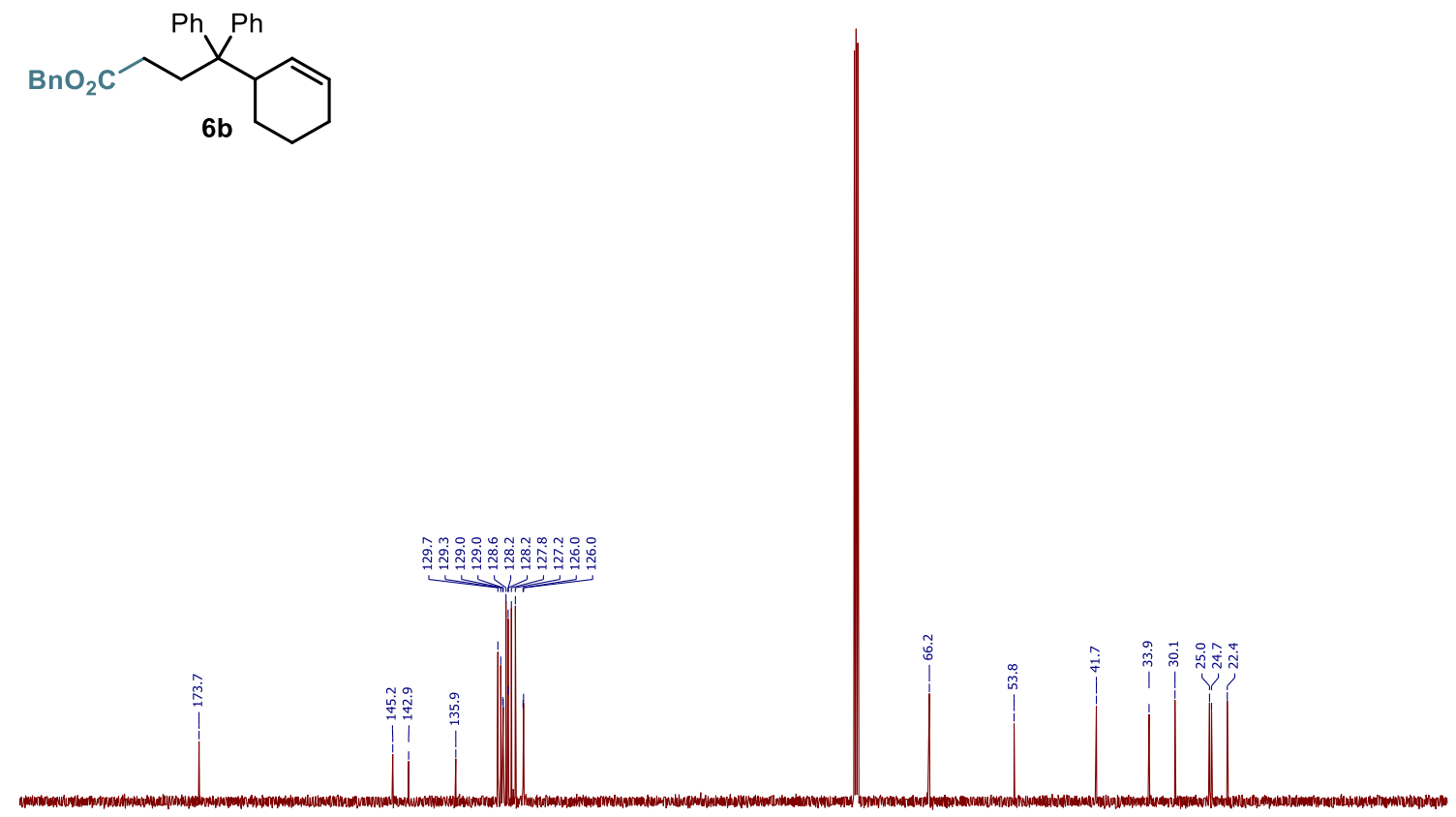

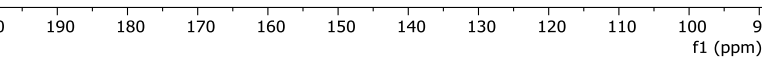

${ }^{1} \mathrm{H}$ NMR $\left(400 \mathrm{MHz}, \mathrm{CDCl}_{3}\right)$ of $\mathbf{6 c}$<smiles>N#CCCC(c1ccccc1)(c1ccccc1)C1C=CCCC1</smiles>

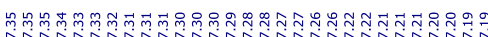

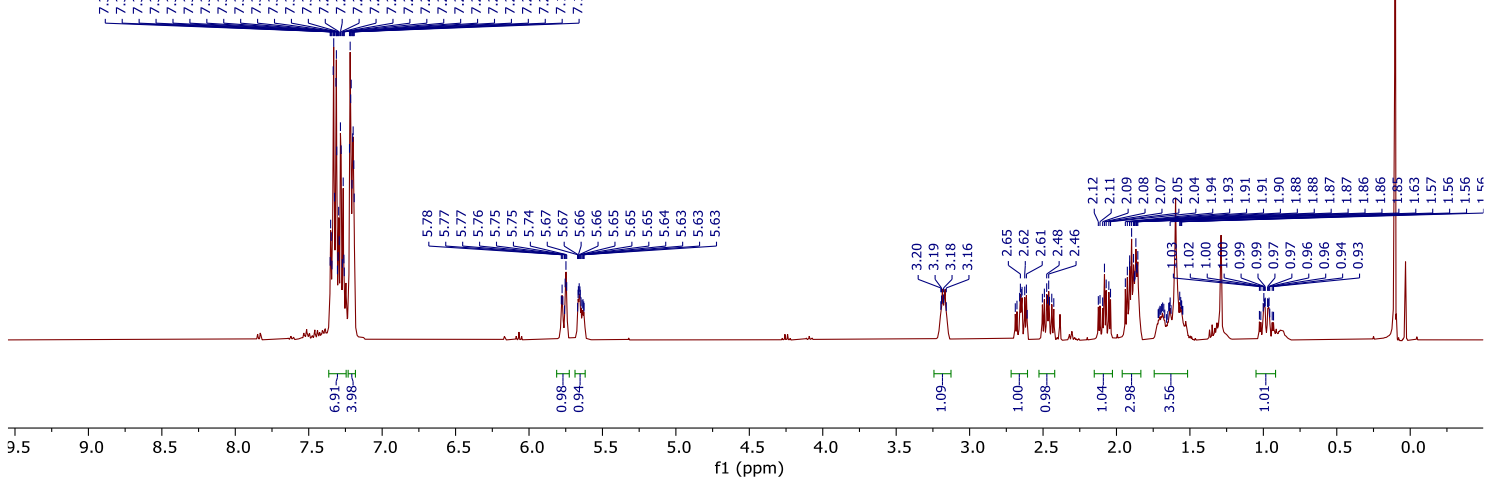

${ }^{13} \mathrm{C}$ NMR $\left(101 \mathrm{MHz}, \mathrm{CDCl}_{3}\right)$ of $\mathbf{6 c}$ 


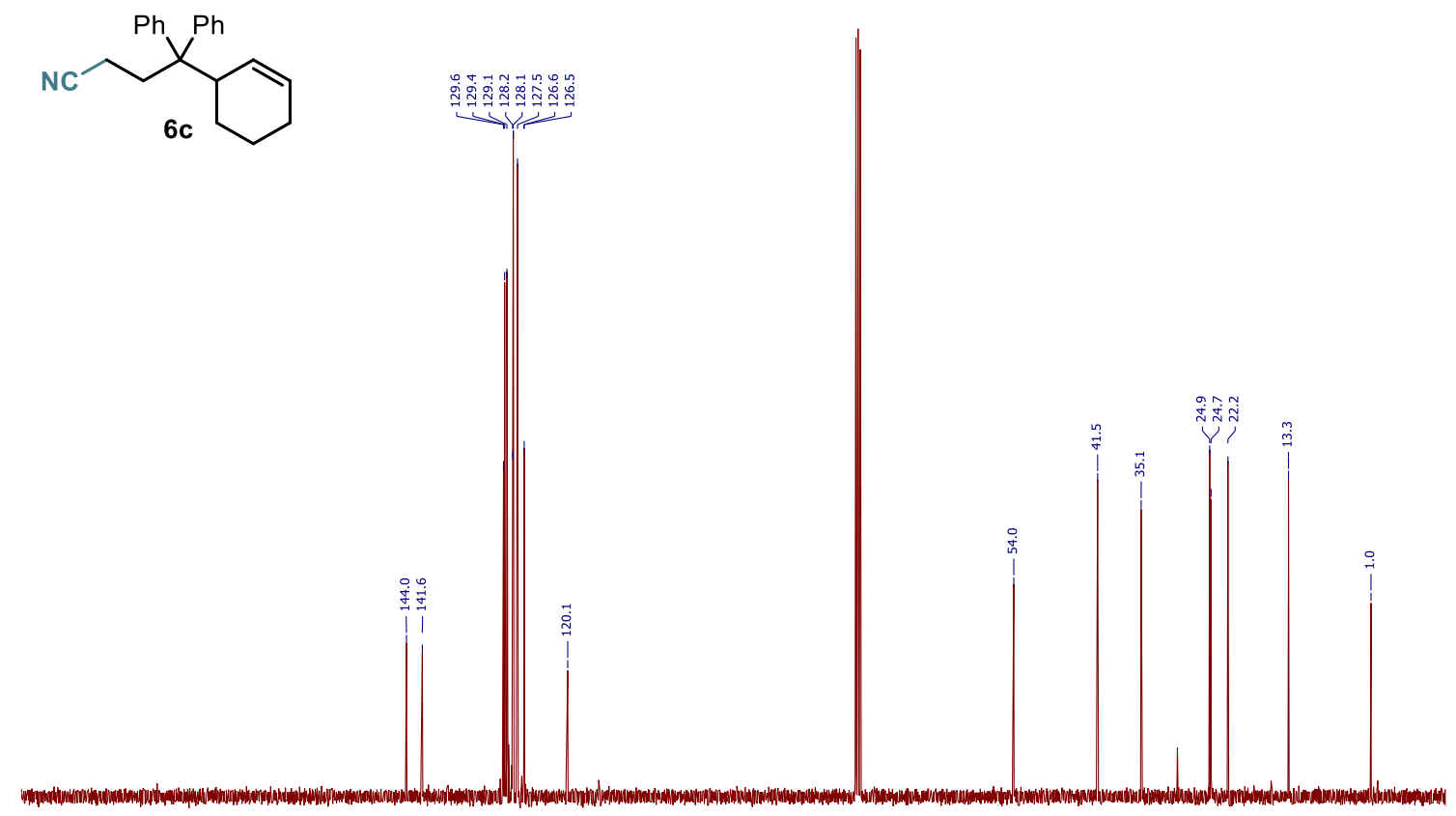

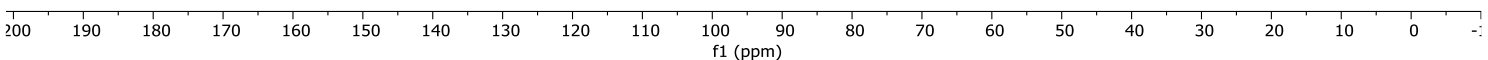

${ }^{1} \mathrm{H}$ NMR (400 MHz, $\mathrm{CDCl}_{3}$ ) of $\mathbf{6 d}$

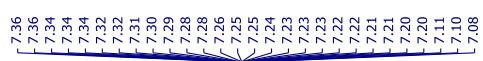

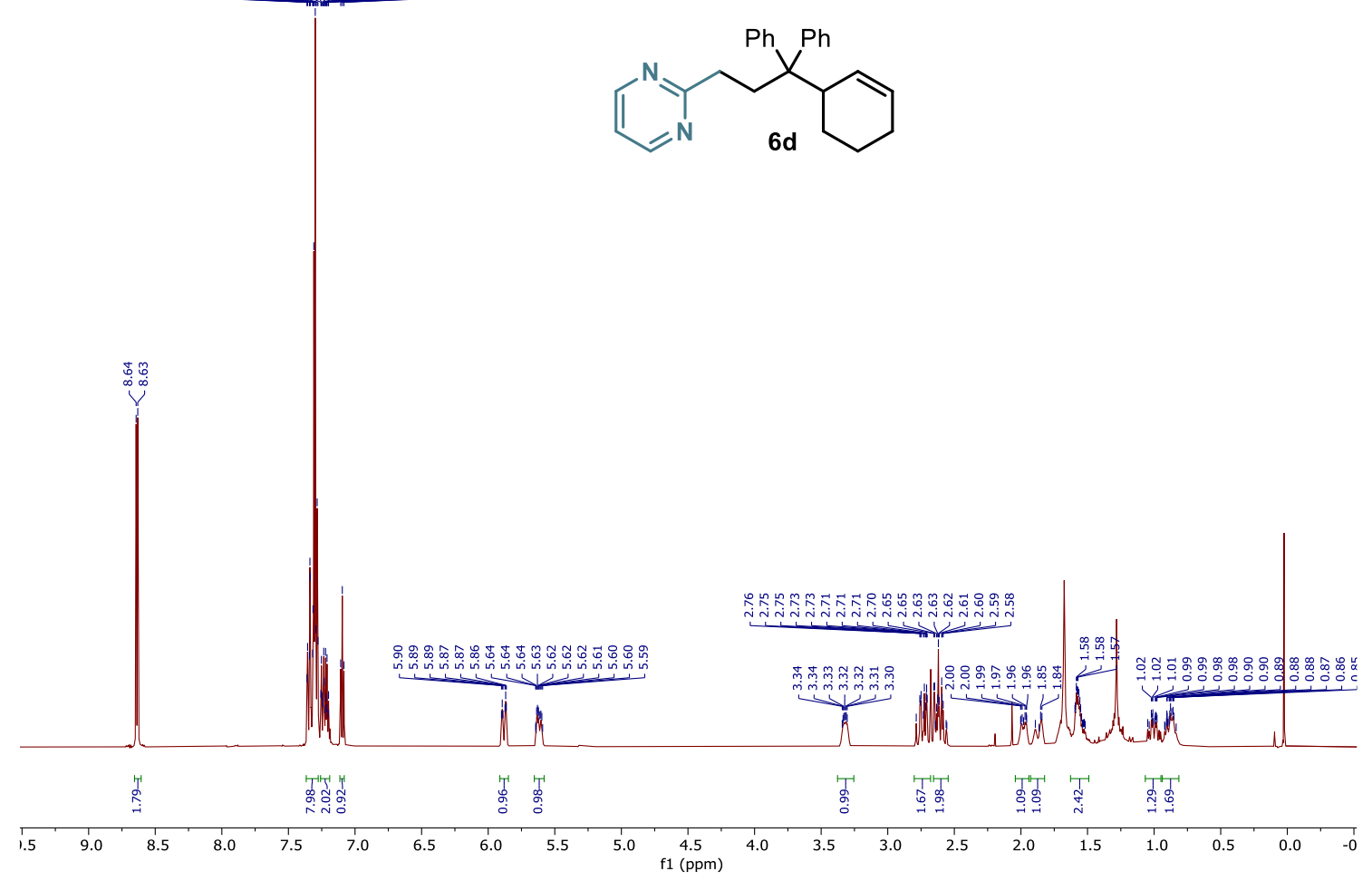

${ }^{13} \mathrm{C}$ NMR (101 MHz, $\left.\mathrm{CDCl}_{3}\right)$ of $\mathbf{6 d}$ 

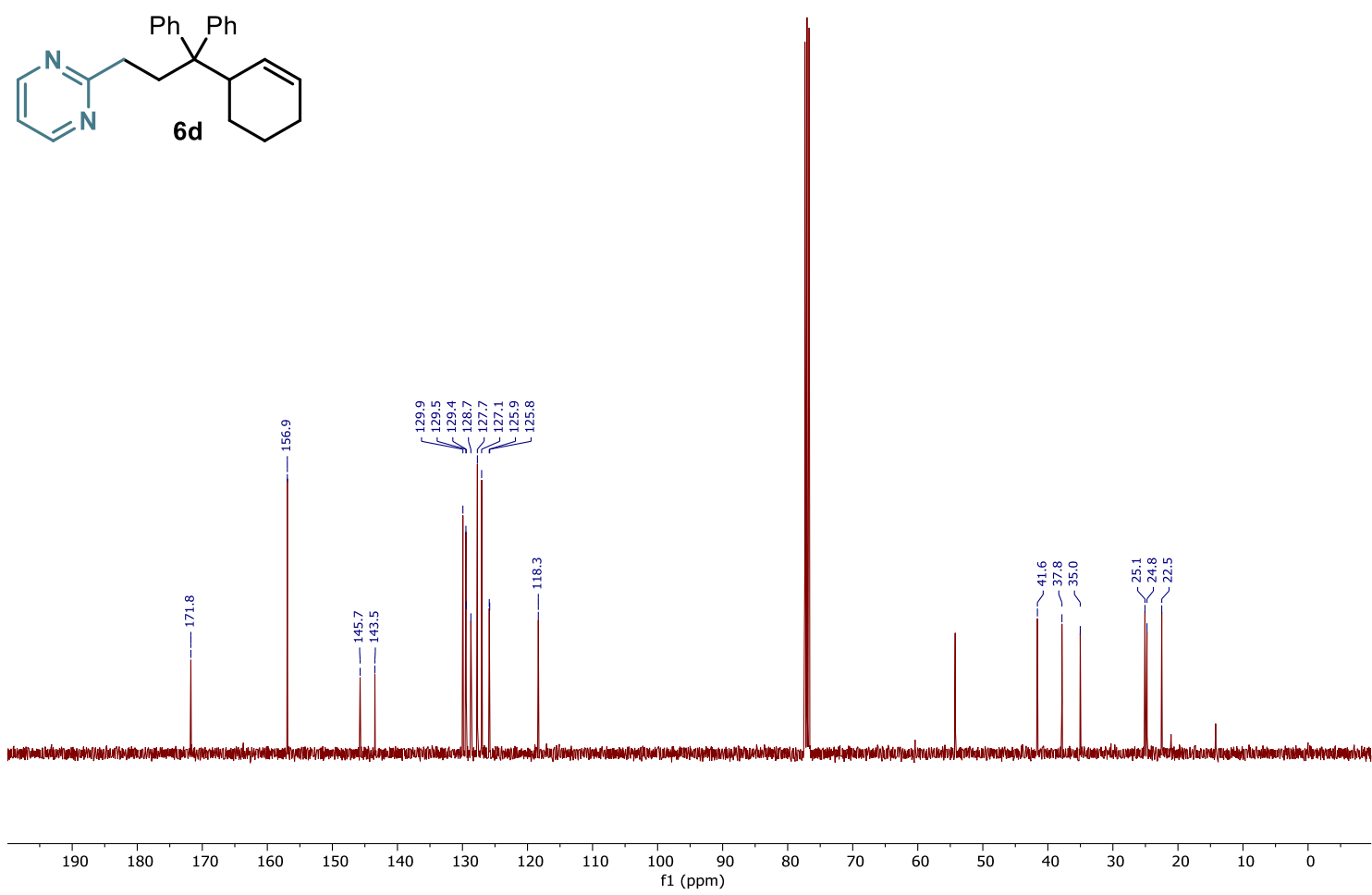

${ }^{1} \mathrm{H}$ NMR (400 MHz, $\left.\mathrm{CDCl}_{3}\right)$ of $\mathbf{6 e}$

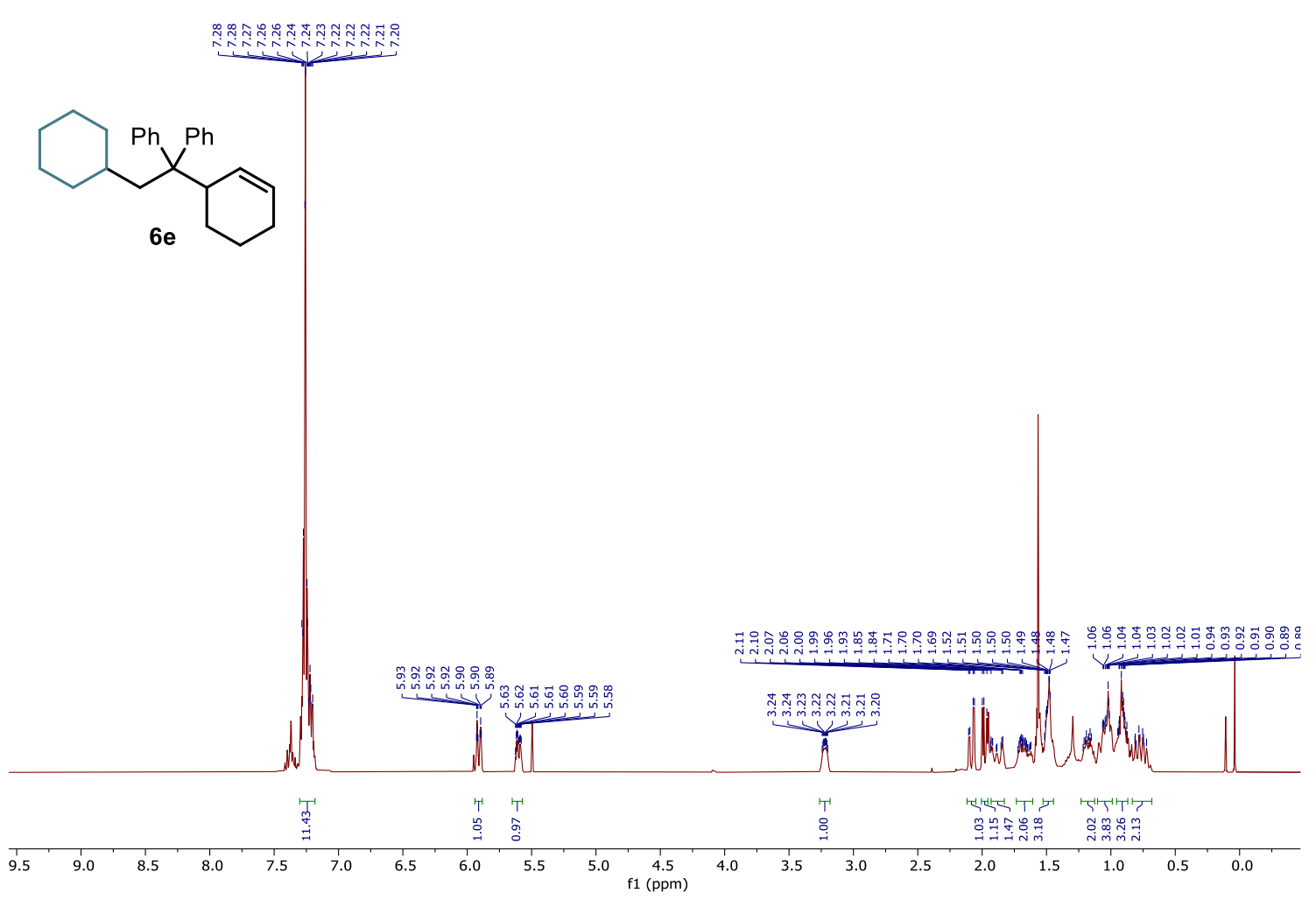

S76 
${ }^{13} \mathrm{C}$ NMR $\left(101 \mathrm{MHz}, \mathrm{CDCl}_{3}\right)$ of $\mathbf{6 e}$
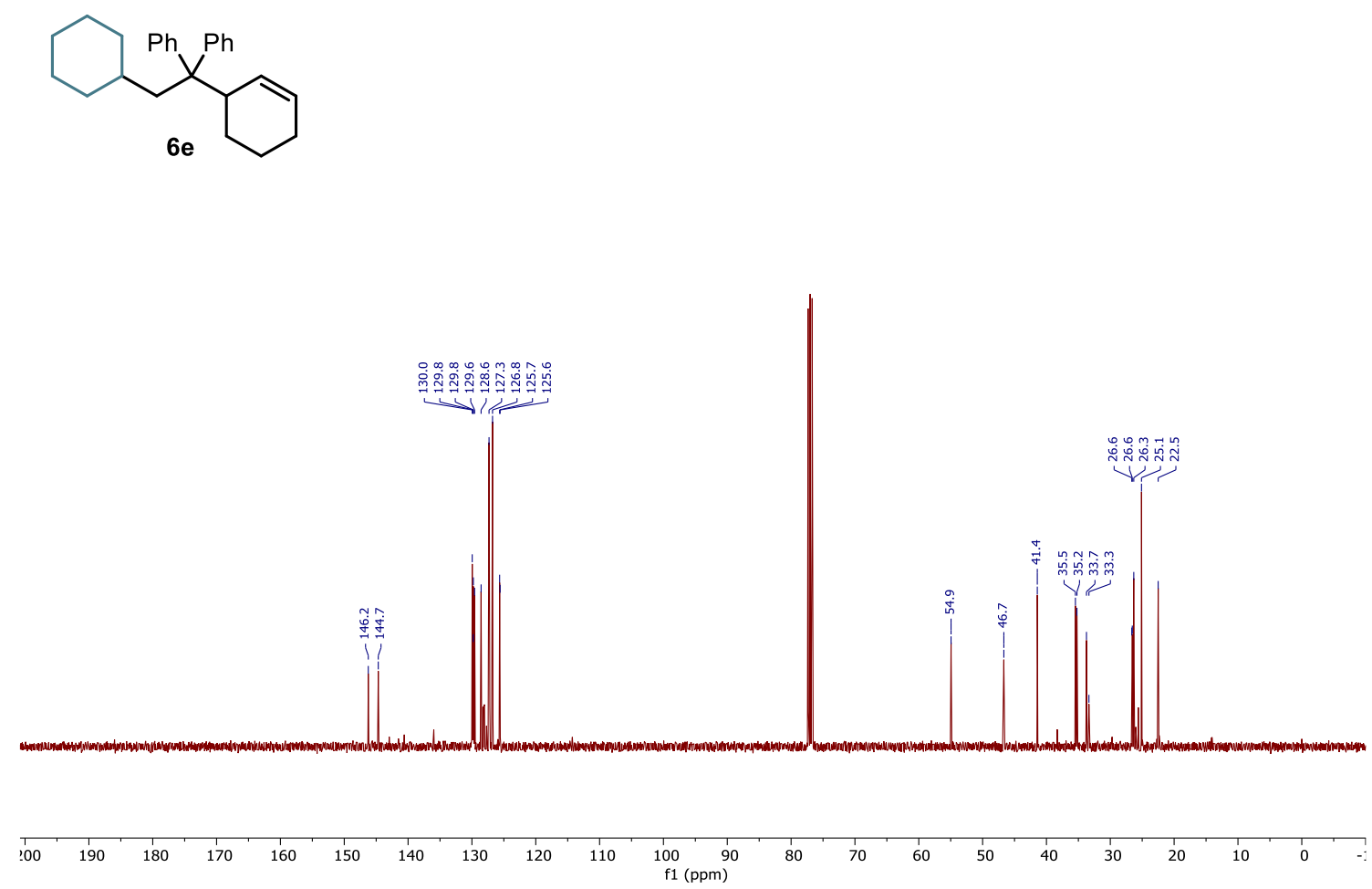

${ }^{1} \mathrm{H}$ NMR (500 MHz, $\left.\mathrm{CDCl}_{3}\right)$ of $6 \mathbf{f}$
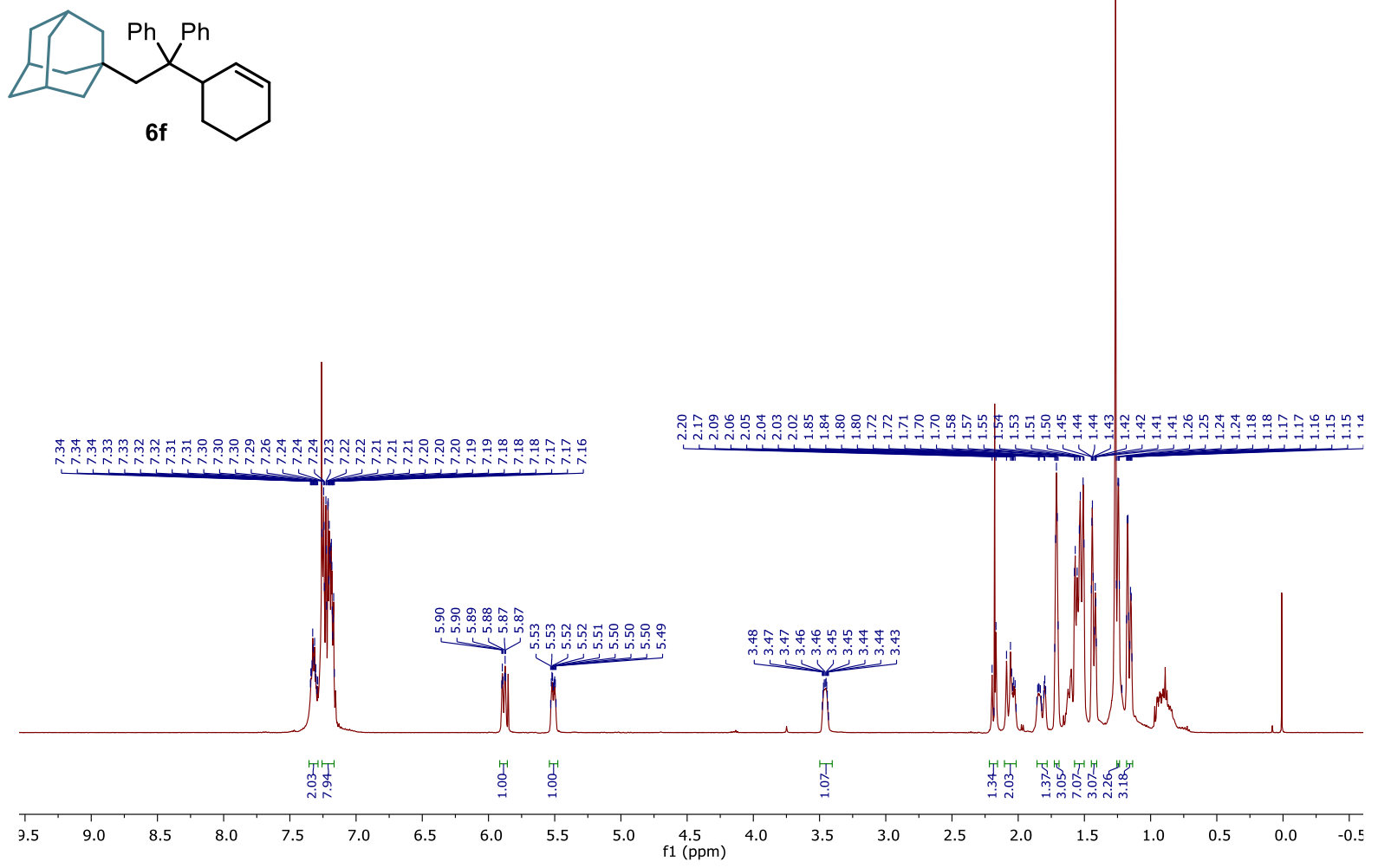

S77 
${ }^{13} \mathrm{C}$ NMR $\left(126 \mathrm{MHz}, \mathrm{CDCl}_{3}\right)$ of $\mathbf{6 f}$
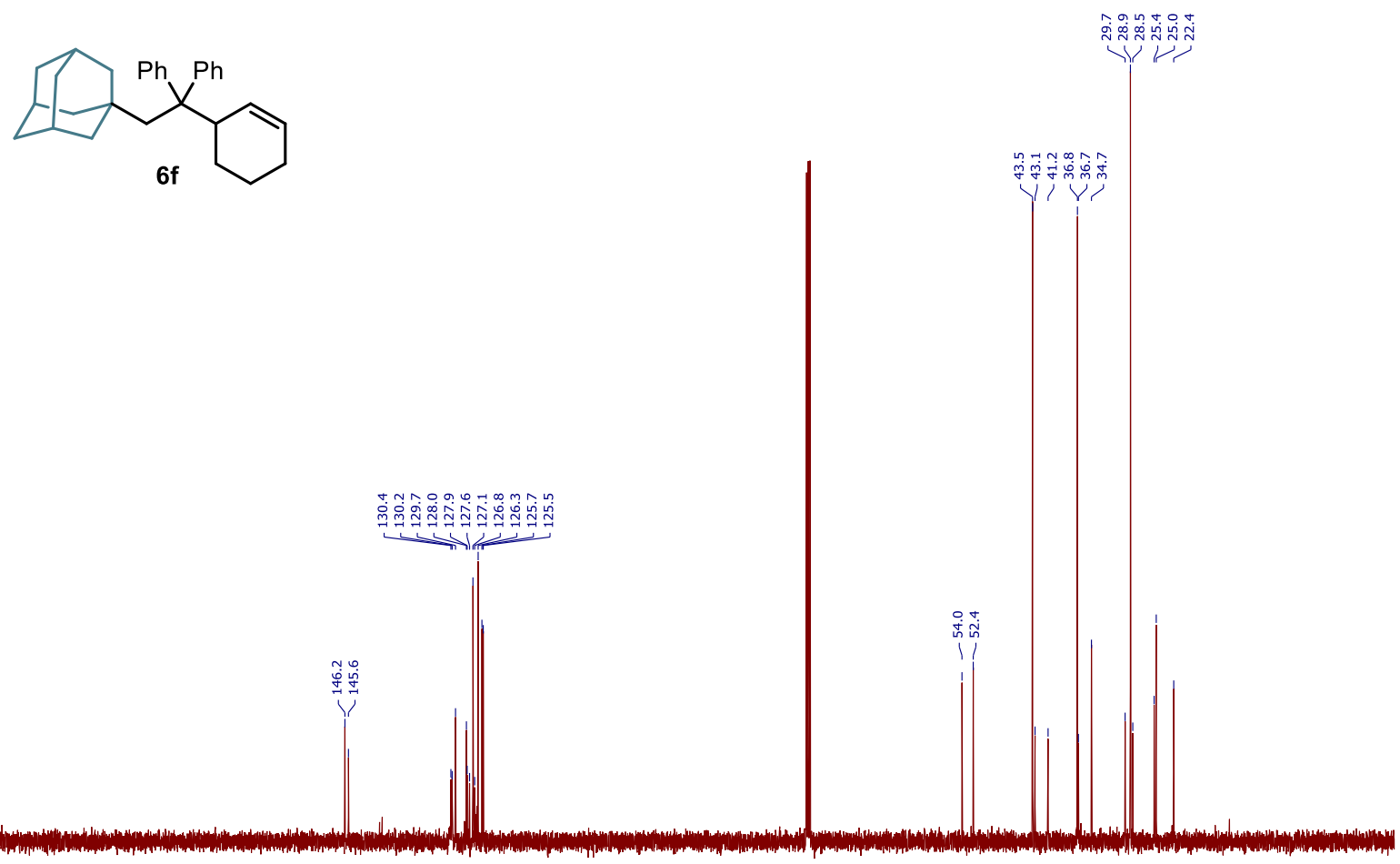

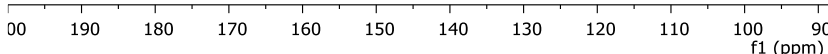

${ }^{1} \mathrm{H} \mathrm{NMR}\left(400 \mathrm{MHz}, \mathrm{CDCl}_{3}\right.$ ) of $\mathbf{6 g}$ 

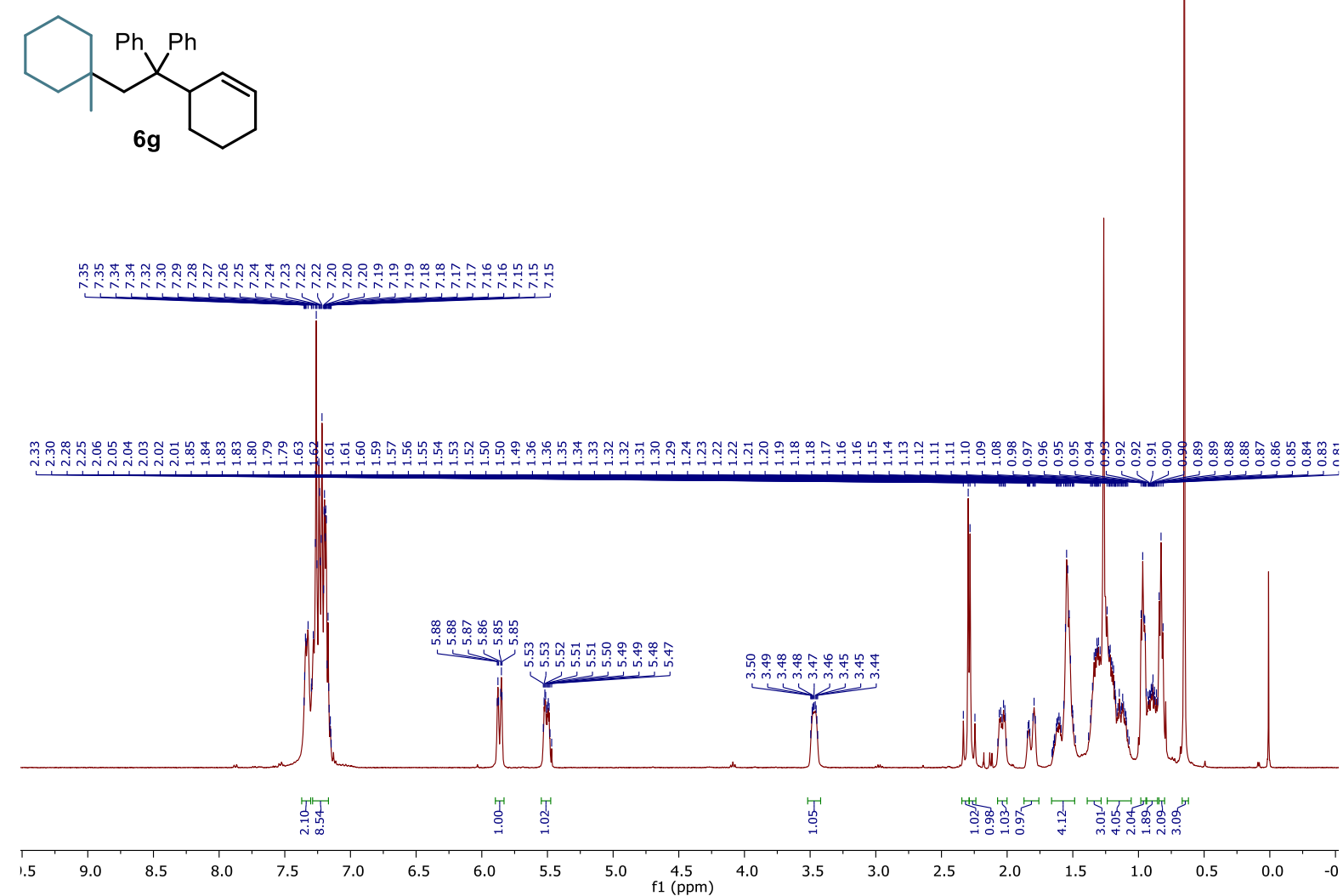

${ }^{13} \mathrm{C} \mathrm{NMR}\left(101 \mathrm{MHz}, \mathrm{CDCl}_{3}\right)$ of $\mathbf{6 g}$
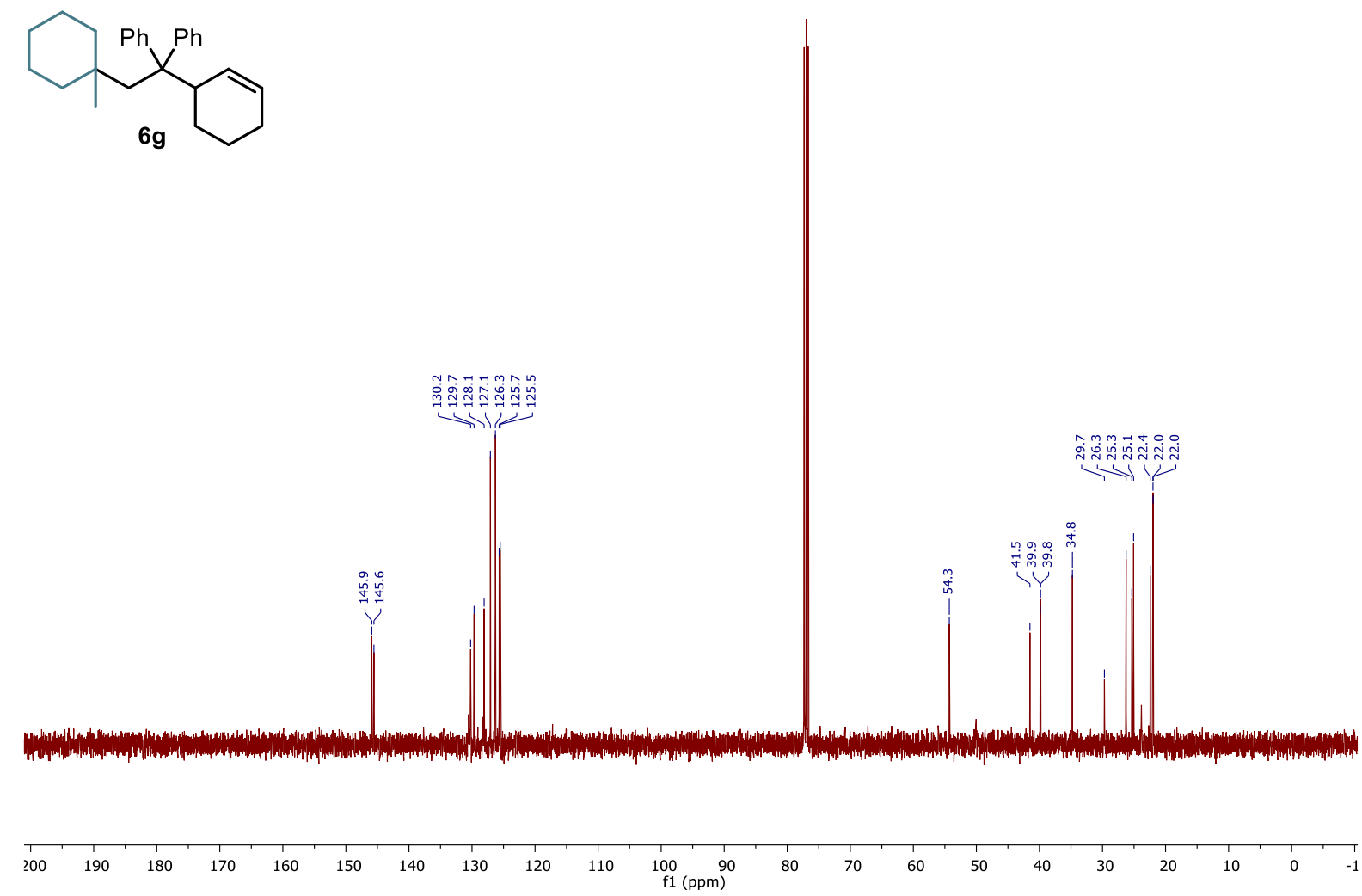

${ }^{1} \mathrm{H}$ NMR $\left(400 \mathrm{MHz}, \mathrm{CDCl}_{3}\right)$ of $\mathbf{6 h}$ 


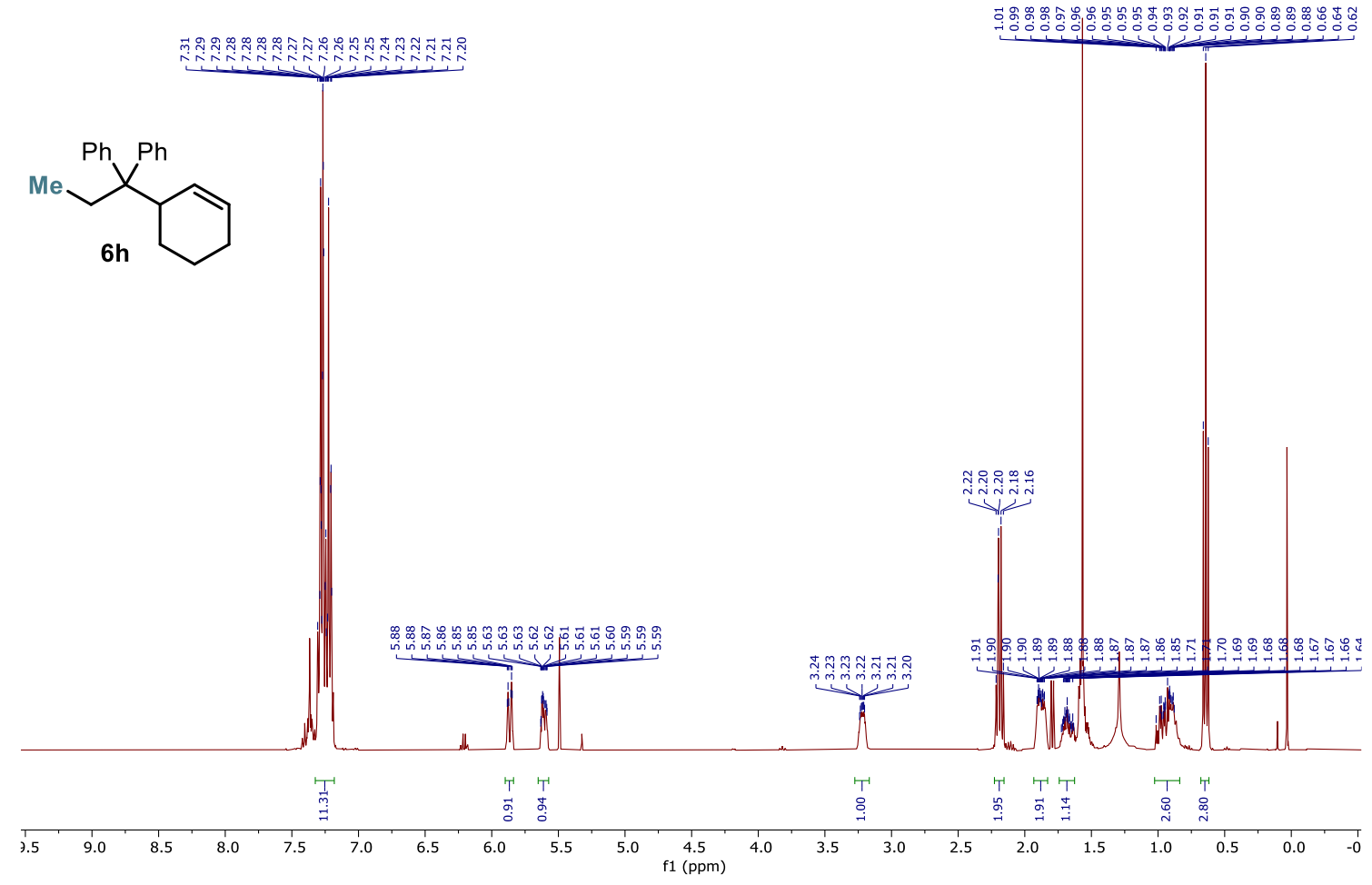

${ }^{13} \mathrm{C}$ NMR (101 MHz, $\mathrm{CDCl}_{3}$ ) of $\mathbf{6 h}$<smiles>CCC(c1ccccc1)(c1ccccc1)C1C=CCCC1</smiles>

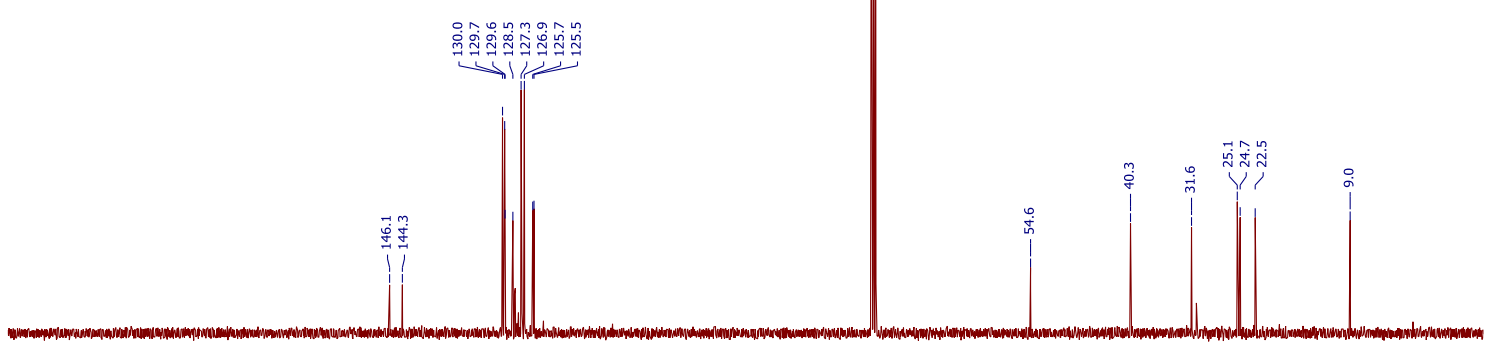

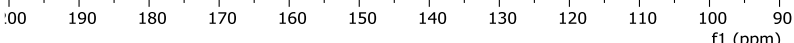

${ }^{1} \mathrm{H}$ NMR $\left(500 \mathrm{MHz}, \mathrm{CDCl}_{3}\right)$ of $\mathbf{6 i}$ 


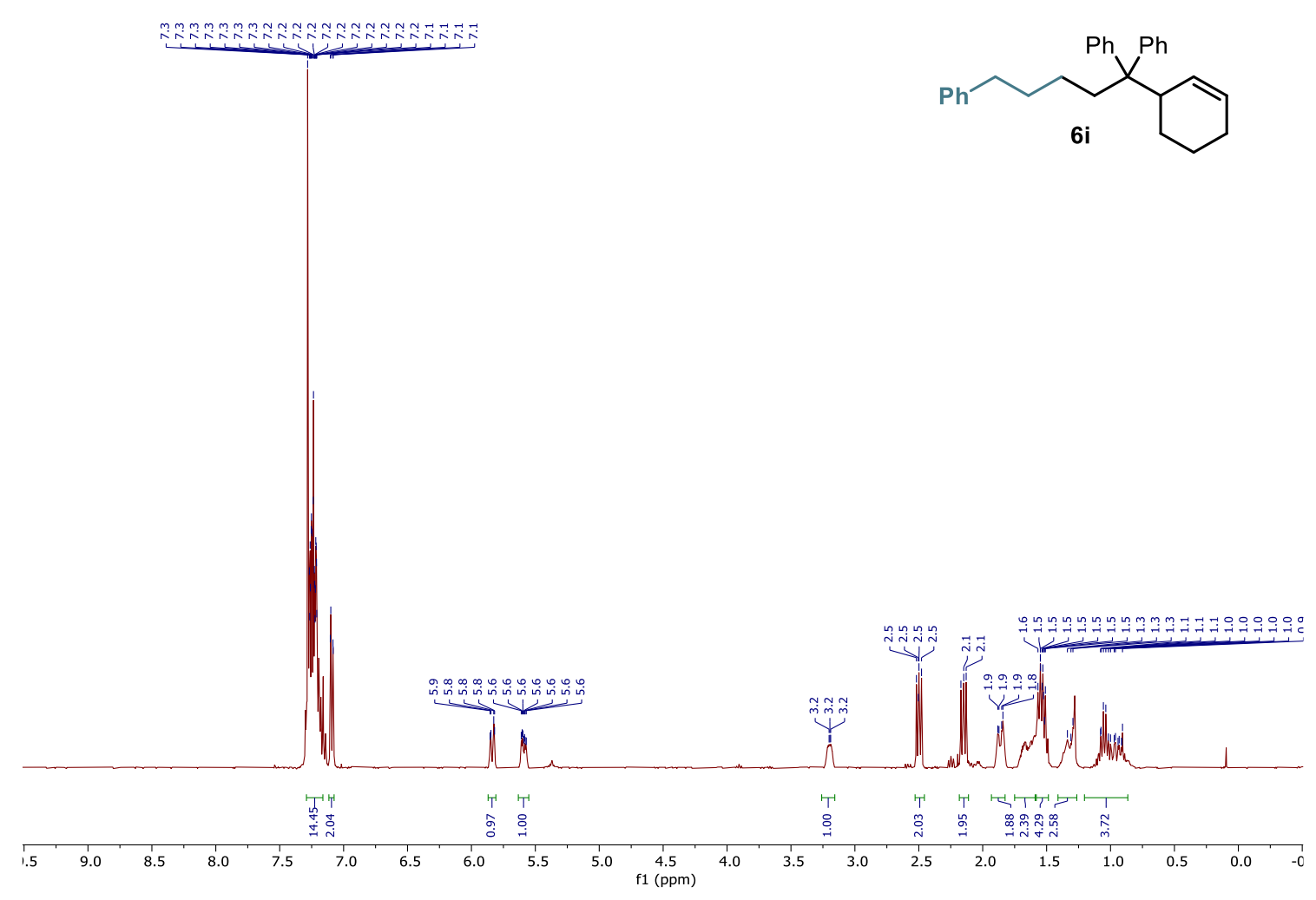

${ }^{13} \mathrm{C} \mathrm{NMR}\left(101 \mathrm{MHz}, \mathrm{CDCl}_{3}\right)$ of $\mathbf{6 i}$
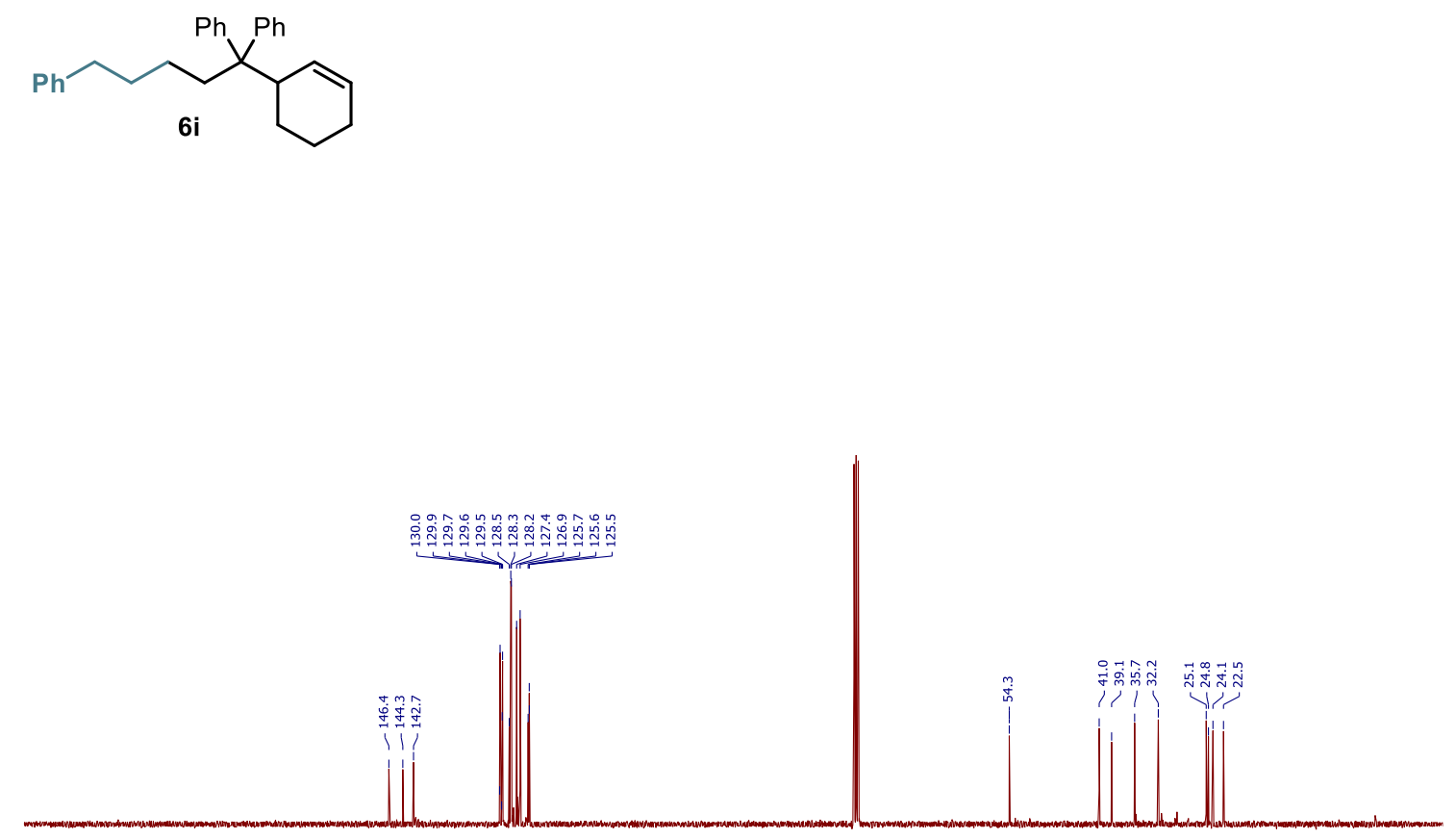

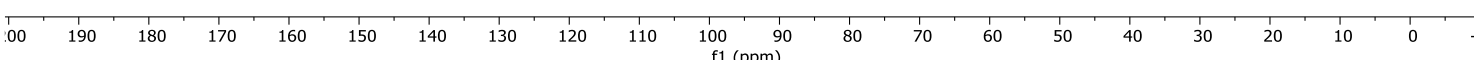

${ }^{1} \mathrm{H}$ NMR $\left(500 \mathrm{MHz}, \mathrm{CDCl}_{3}\right)$ of $\mathbf{6 j}$ 


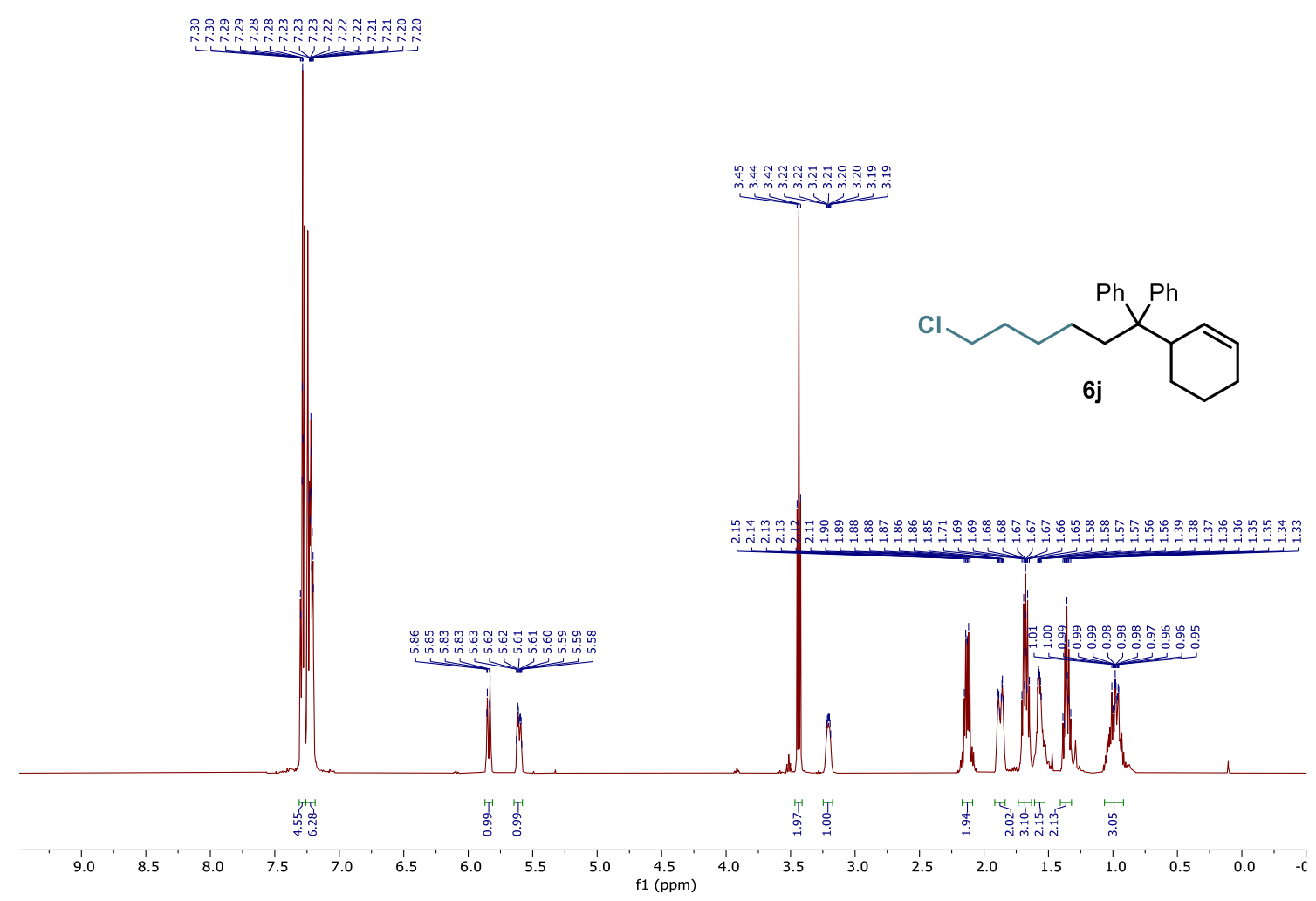

${ }^{13} \mathrm{C} \mathrm{NMR}\left(126 \mathrm{MHz}, \mathrm{CDCl}_{3}\right)$ of $\mathbf{6 j}$

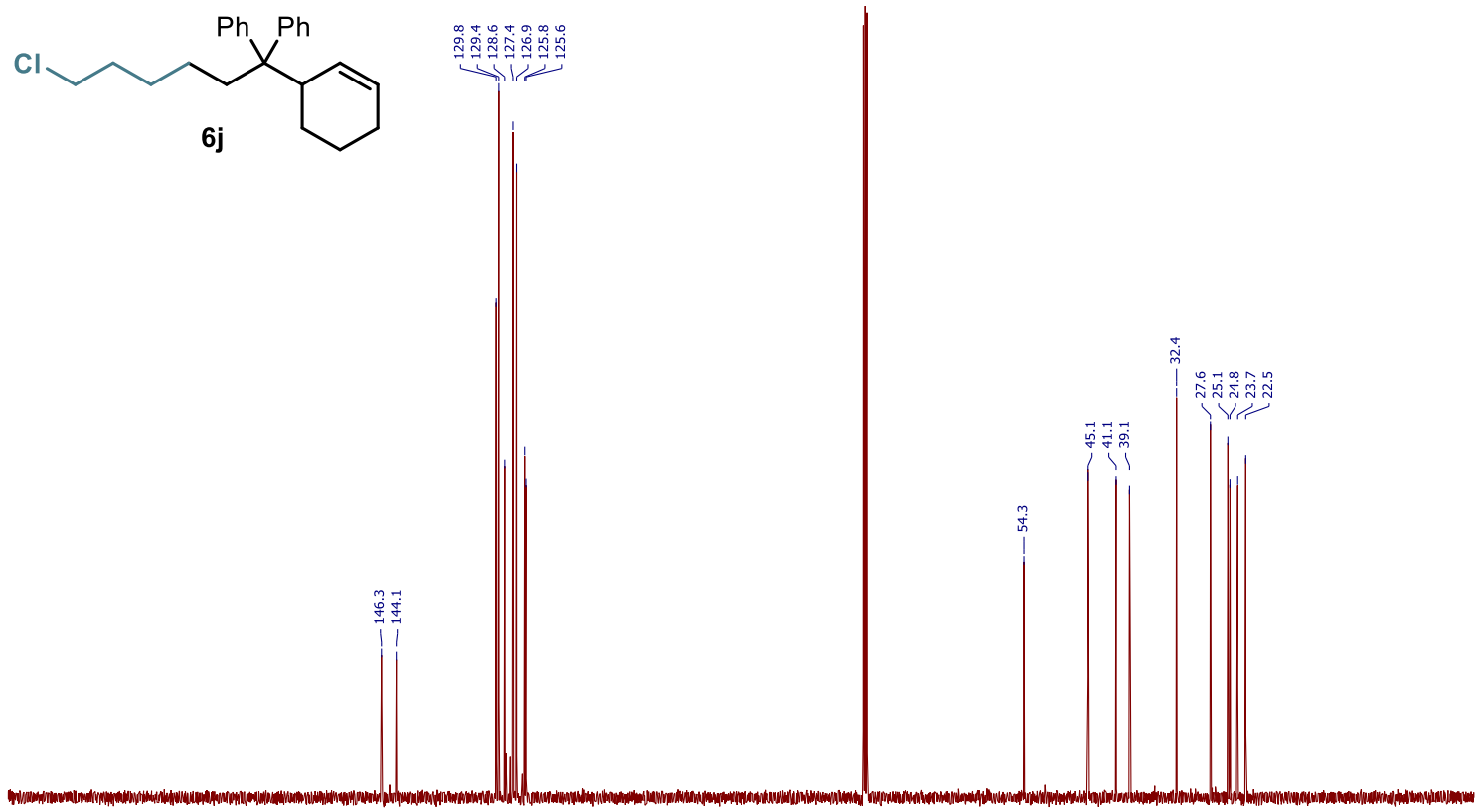

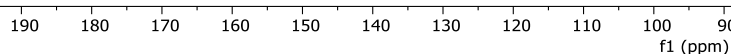

${ }^{1} \mathrm{H}$ NMR $\left(500 \mathrm{MHz}, \mathrm{CDCl}_{3}\right)$ of $\mathbf{6 k}$ 

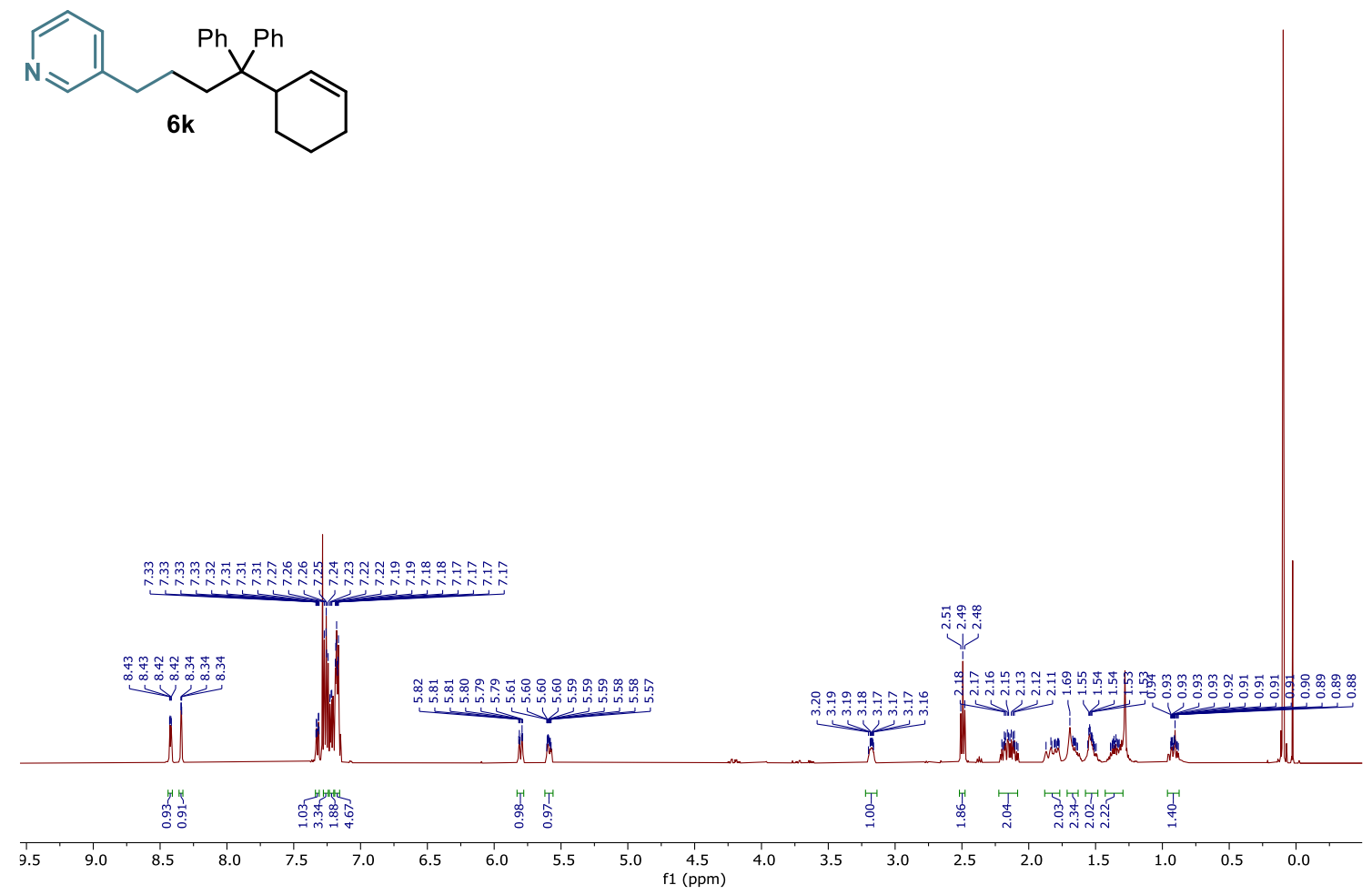

${ }^{13} \mathrm{C} \mathrm{NMR}\left(101 \mathrm{MHz}, \mathrm{CDCl}_{3}\right)$ of $\mathbf{6 j}$<smiles>C1=CC(C(CCCc2cccnc2)(c2ccccc2)c2ccccc2)CCC1</smiles>

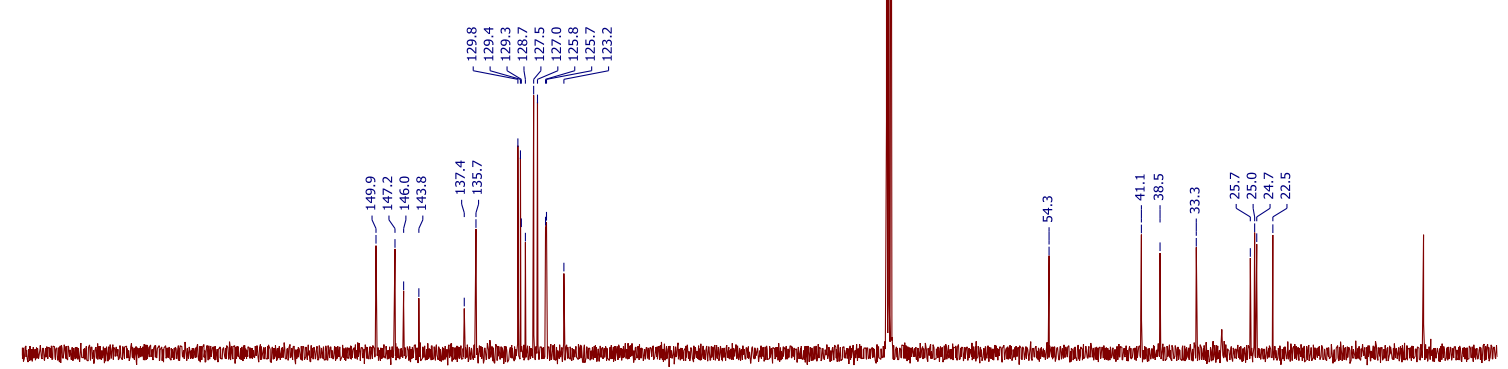

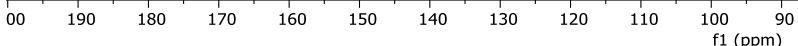

${ }^{1} \mathrm{H}$ NMR $\left(500 \mathrm{MHz}, \mathrm{CDCl}_{3}\right)$ of $\mathbf{6}$ 


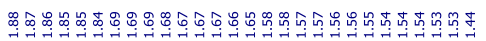
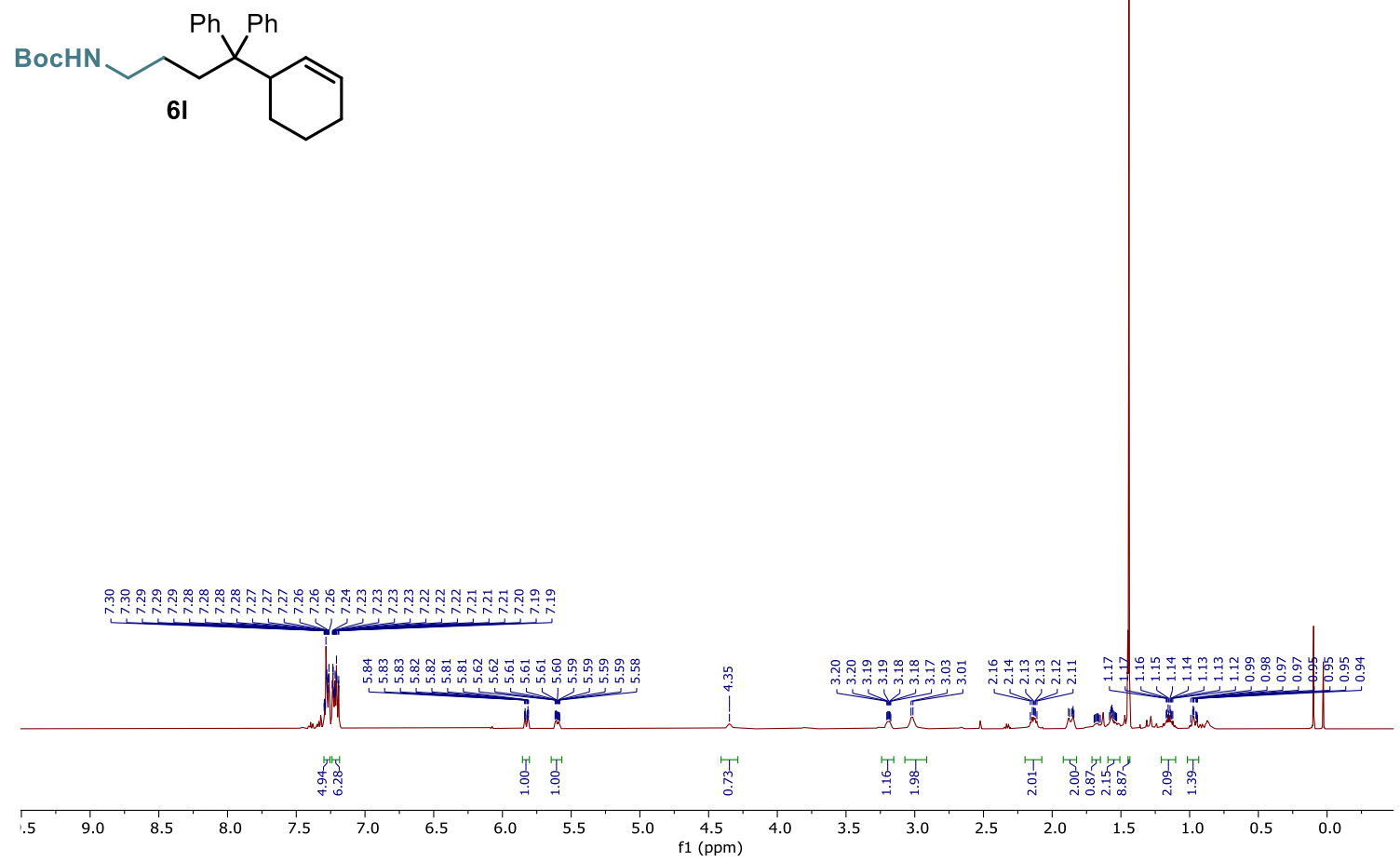

${ }^{13} \mathrm{C}$ NMR $\left(101 \mathrm{MHz}, \mathrm{CDCl}_{3}\right)$ of $\mathbf{6}$
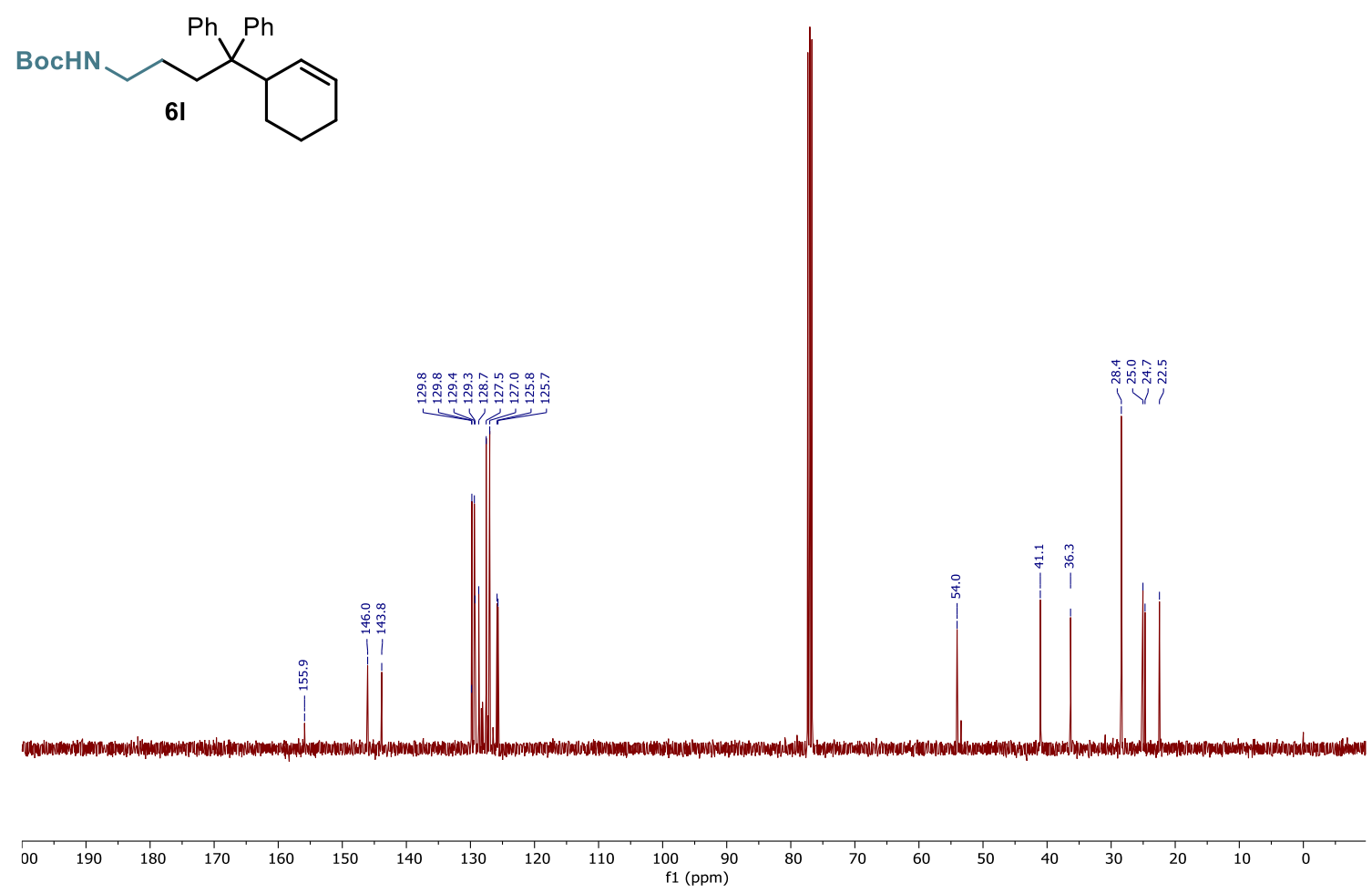

S84 
${ }^{1} \mathrm{H}$ NMR $\left(400 \mathrm{MHz}, \mathrm{CDCl}_{3}\right)$ of $\mathbf{6 m}$

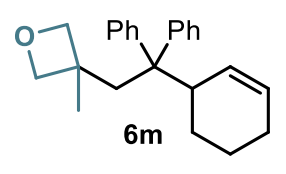

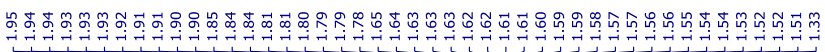

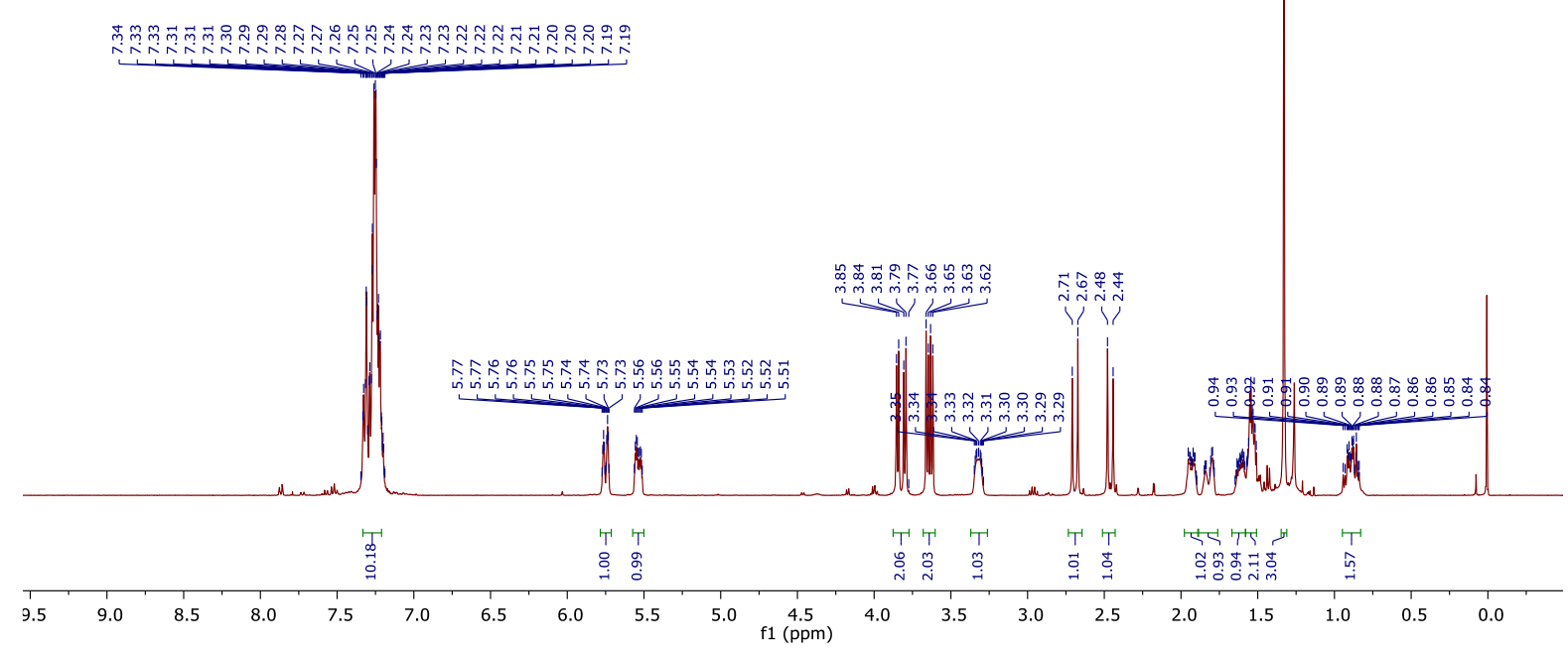

${ }^{13} \mathrm{C}$ NMR (101 MHz, $\left.\mathrm{CDCl}_{3}\right)$ of $\mathbf{6 m}$
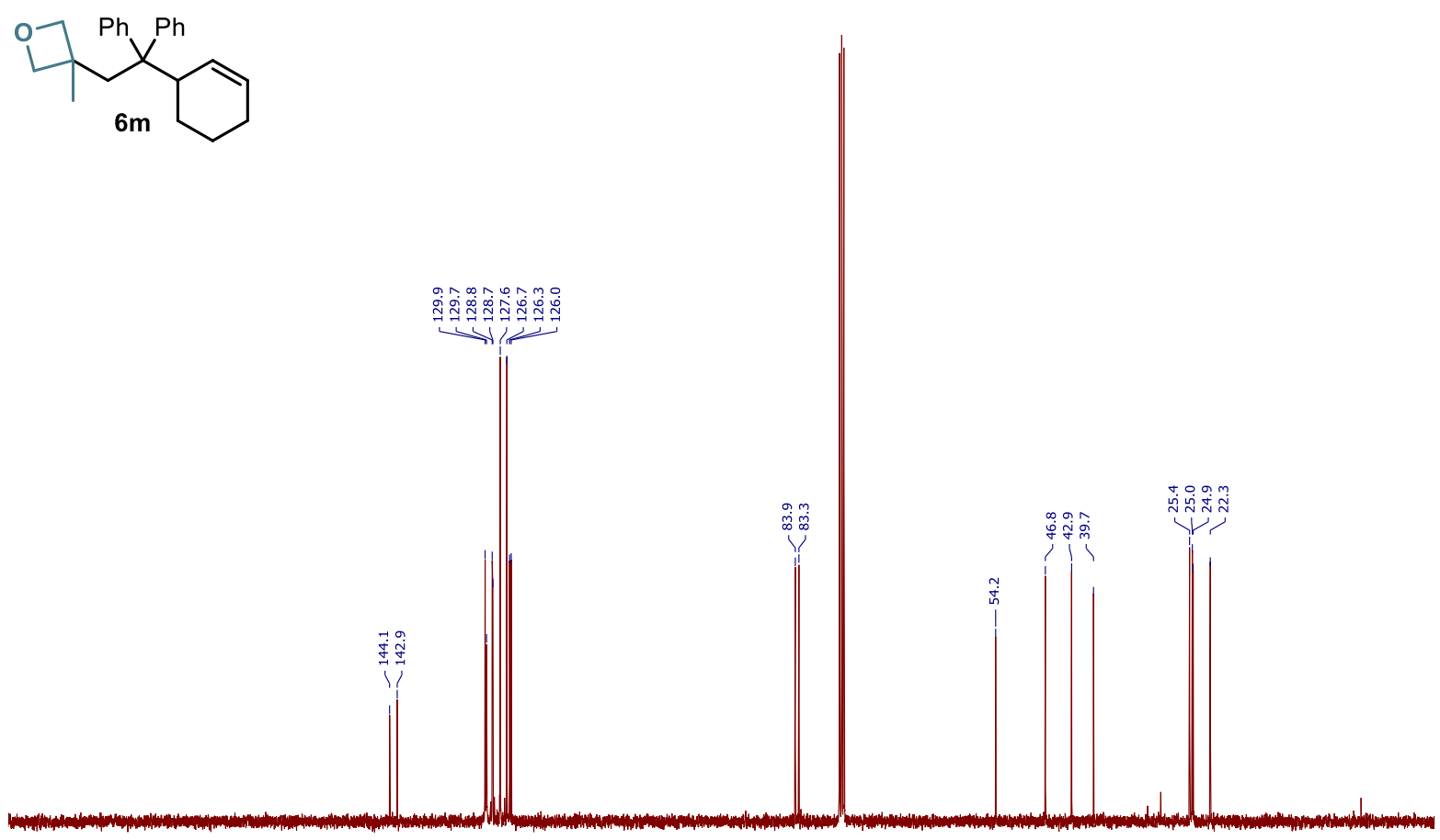

$\begin{array}{lllllllllll}00 & 190 & 180 & 170 & 160 & 150 & 140 & 130 & 120 & 110 & 100 \\ \mathrm{f} 1(\mathrm{ppm})\end{array}$ 
${ }^{1} \mathrm{H}$ NMR $\left(500 \mathrm{MHz}, \mathrm{CDCl}_{3}\right)$ of $\mathbf{6 n}$

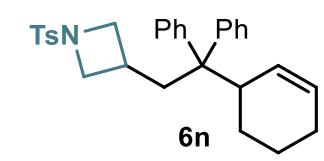

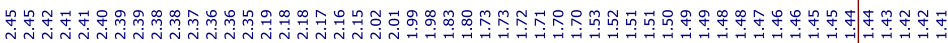

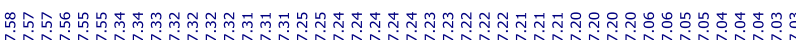

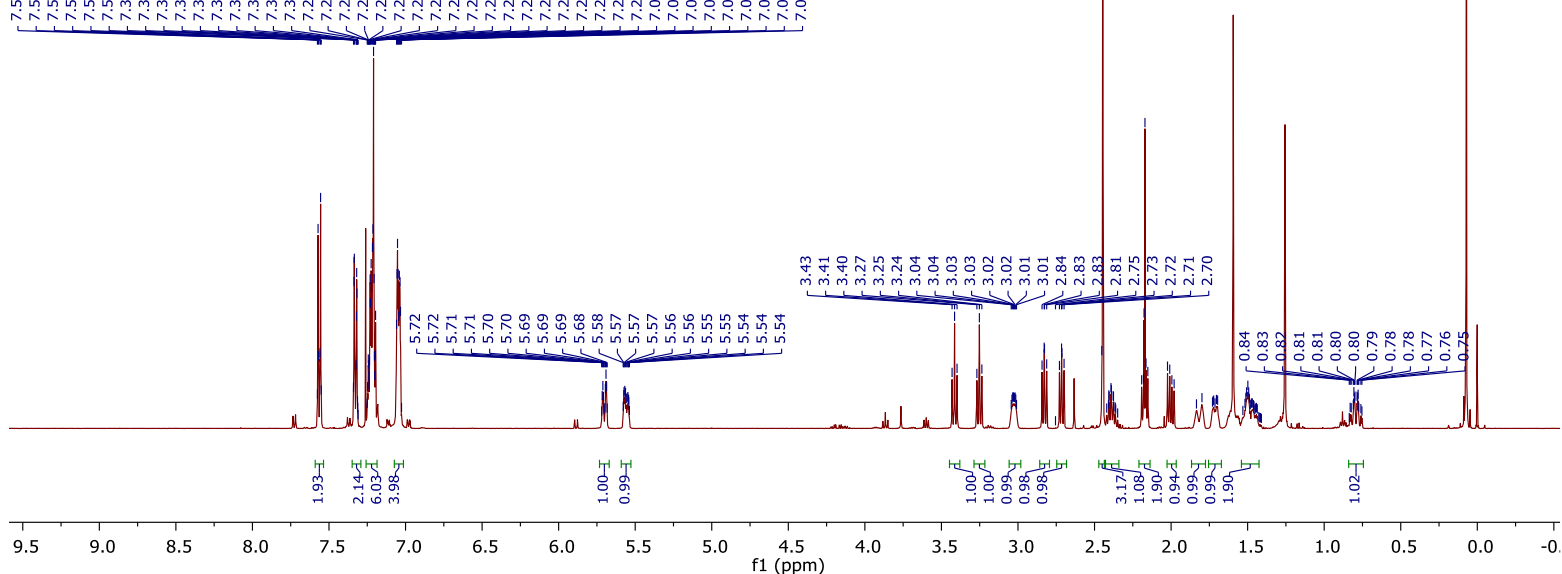

${ }^{13} \mathrm{C}$ NMR $\left(126 \mathrm{MHz}, \mathrm{CDCl}_{3}\right)$ of $\mathbf{6 n}$ 

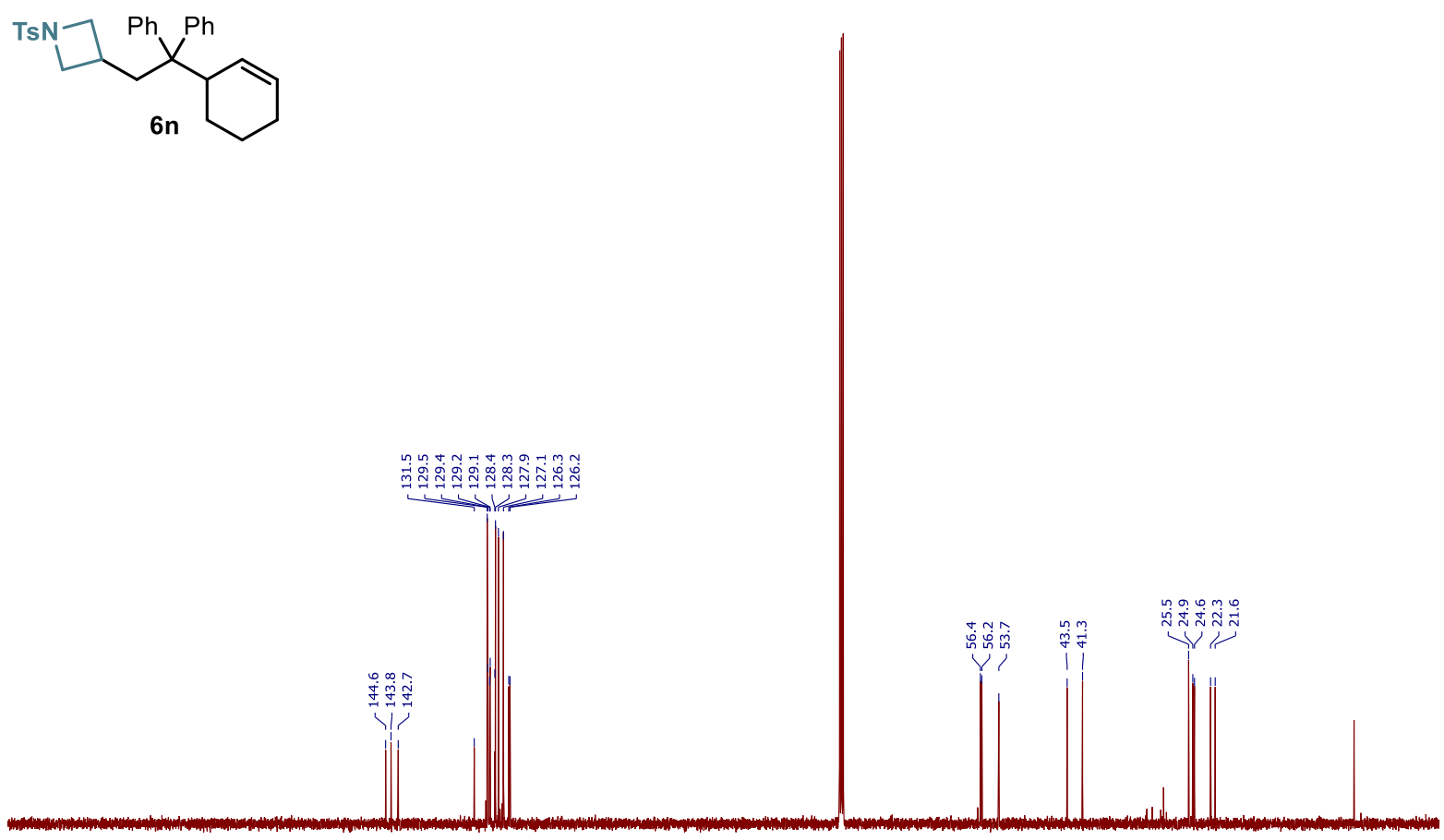

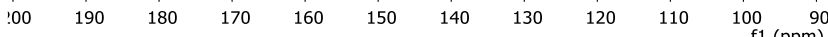

${ }^{1} \mathrm{H}$ NMR $\left(500 \mathrm{MHz}, \mathrm{CDCl}_{3}\right)$ of $\mathbf{6 o}$

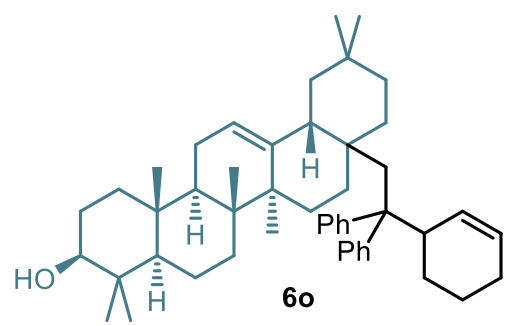

60 

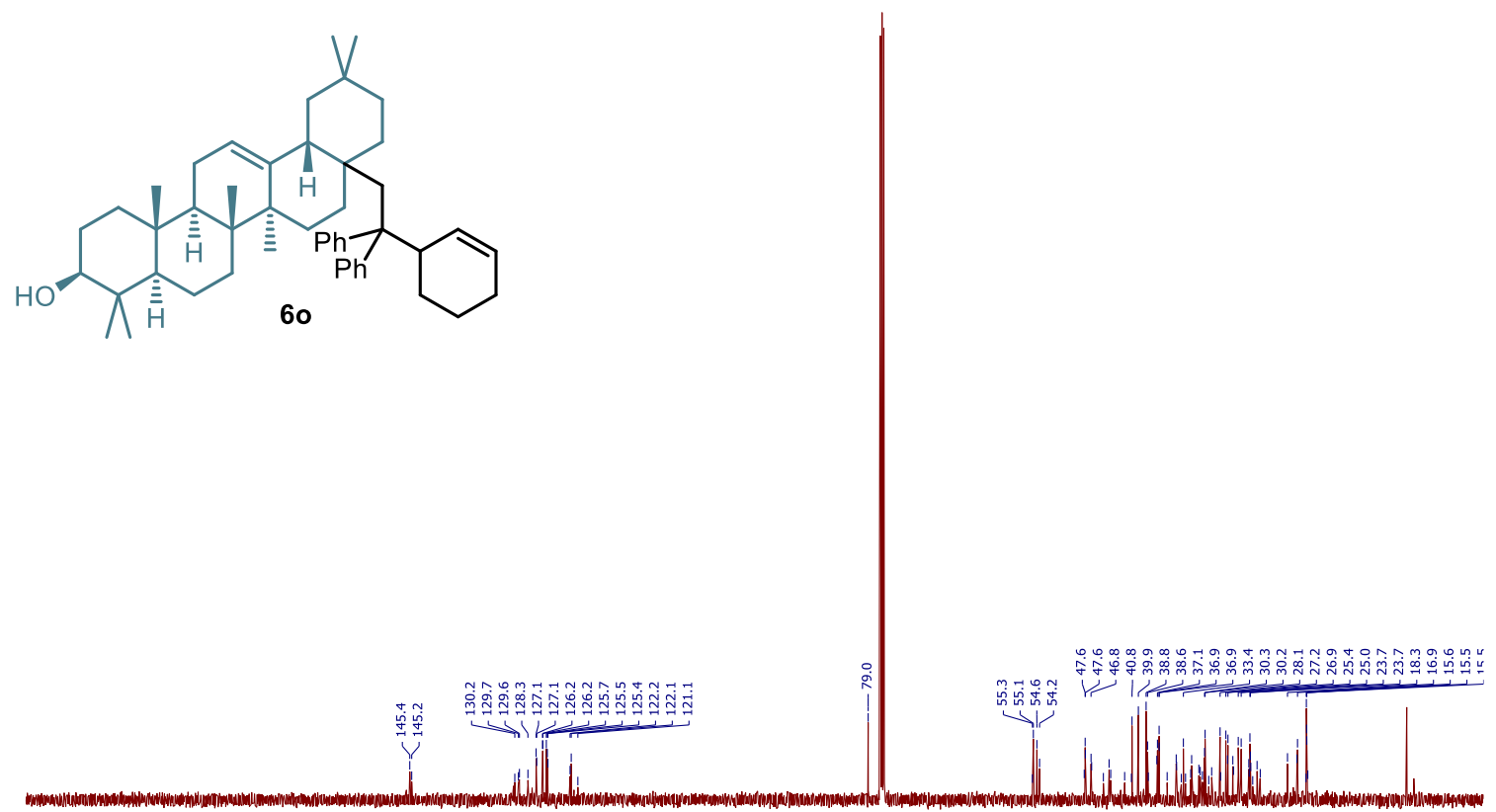

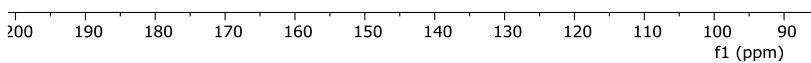

${ }^{1} \mathrm{H}$ NMR $\left(500 \mathrm{MHz}, \mathrm{CDCl}_{3}\right)$ of $\mathbf{6 p}$
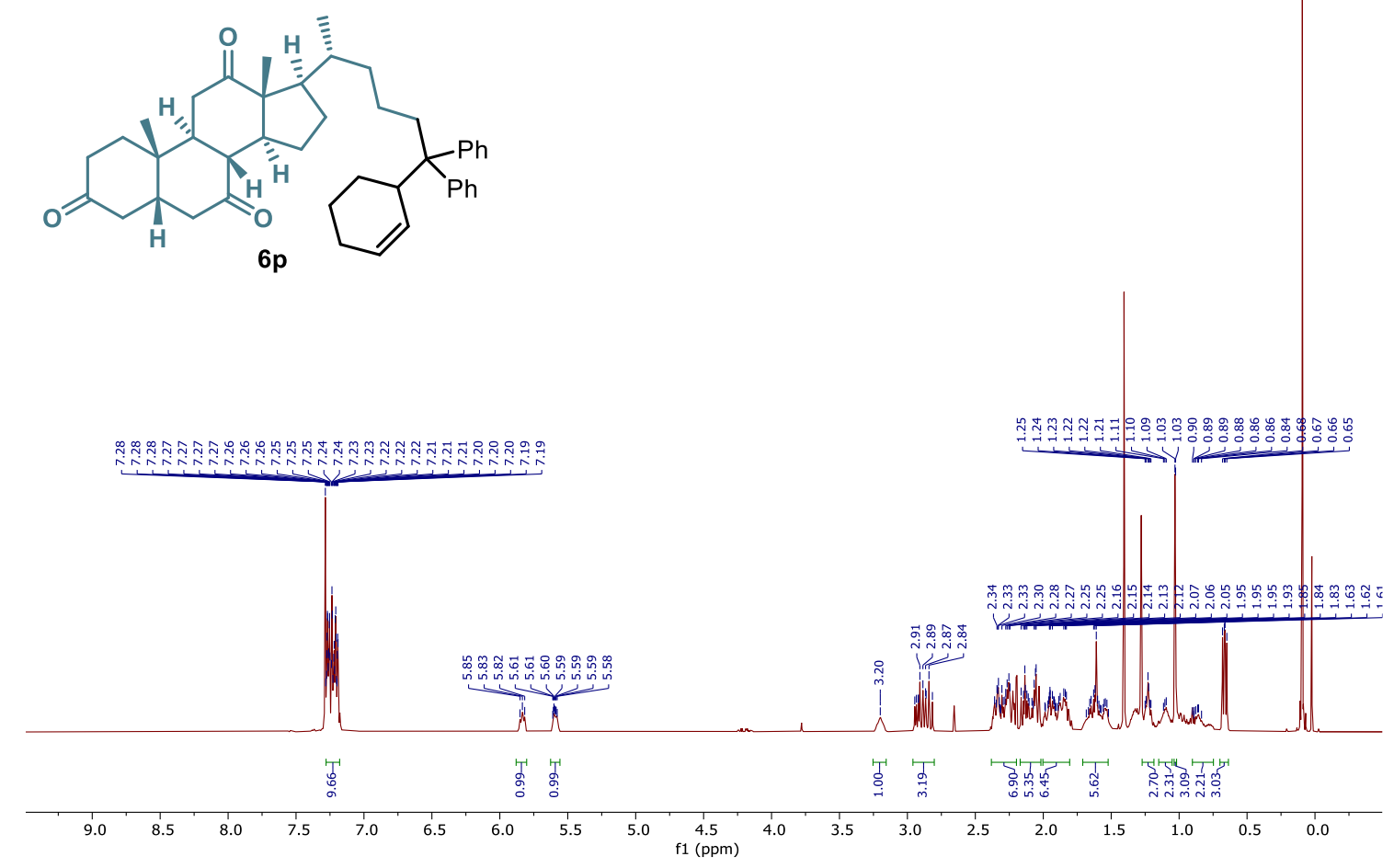

${ }^{13} \mathrm{C}$ NMR $\left(126 \mathrm{MHz}, \mathrm{CDCl}_{3}\right)$ of $\mathbf{6 p}$ 


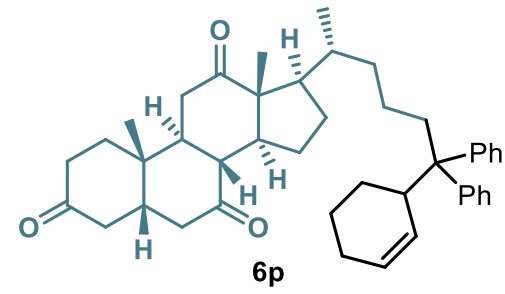

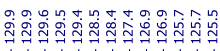

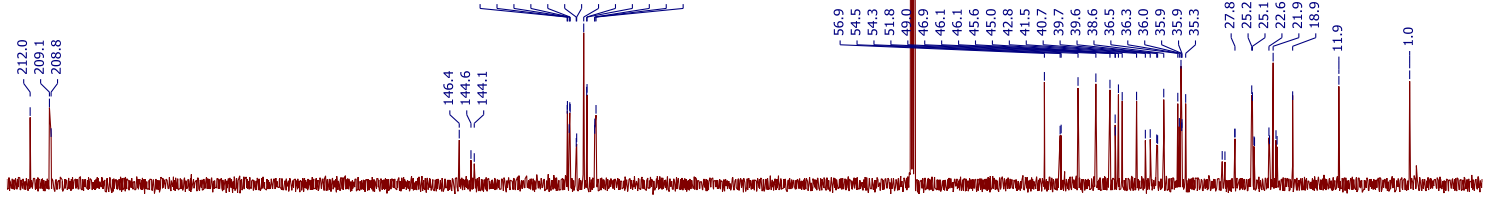

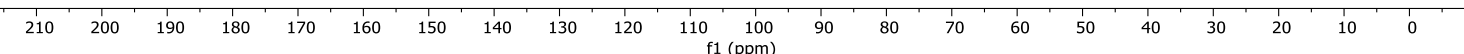


${ }^{1} \mathrm{H}$ NMR $\left(500 \mathrm{MHz}, \mathrm{CDCl}_{3}\right)$ of $\mathbf{6 q}$<smiles></smiles>

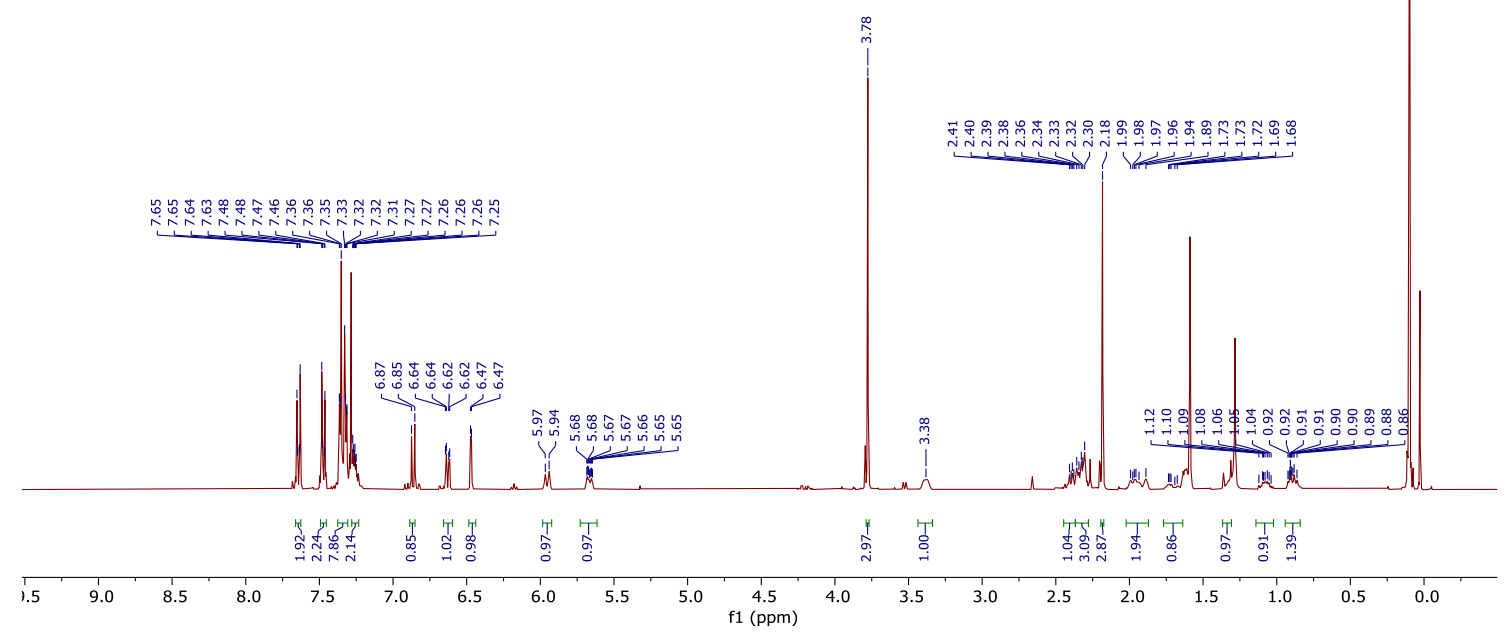

${ }^{13} \mathrm{C}$ NMR (101 MHz, $\left.\mathrm{CDCl}_{3}\right)$ of $\mathbf{6 q}$<smiles></smiles>

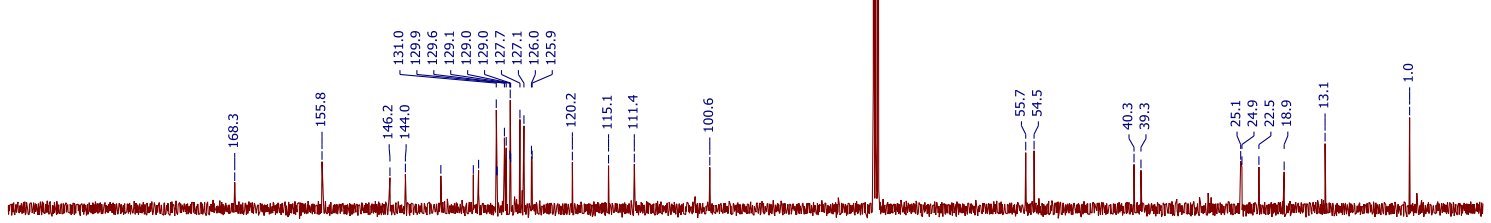

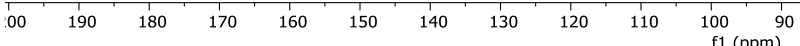


${ }^{1} \mathrm{H}$ NMR (400 MHz, $\left.\mathrm{CDCl}_{3}\right)$ of $\mathbf{6 r}$

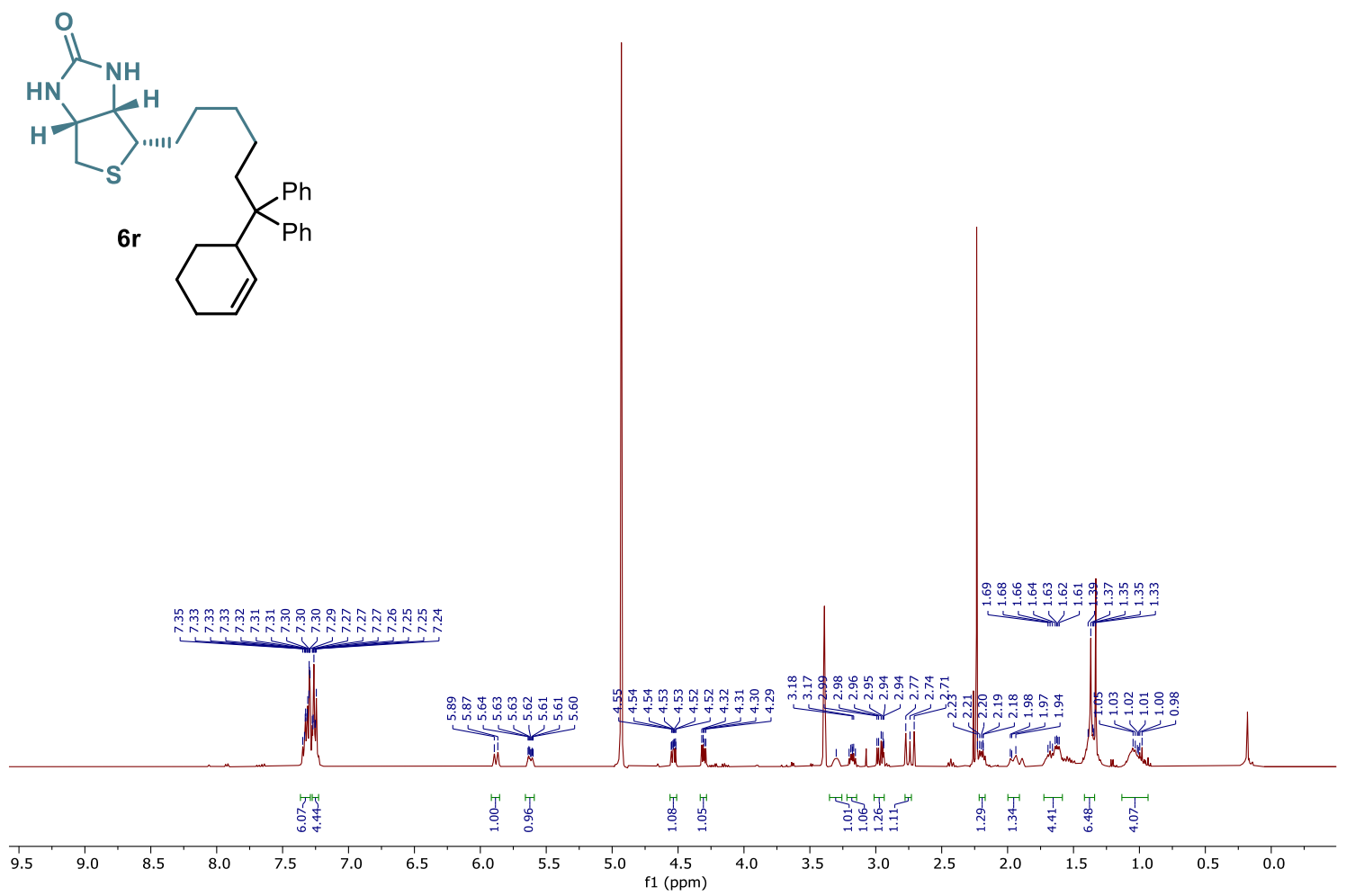

${ }^{13} \mathrm{C}$ NMR (101 MHz, $\left.\mathrm{CDCl}_{3}\right)$ of $\mathbf{6 r}$<smiles>O=C1NC2CS[C@H](CCCCCC(c3ccccc3)(c3ccccc3)C3C=CCCC3)[C@H]2N1</smiles>

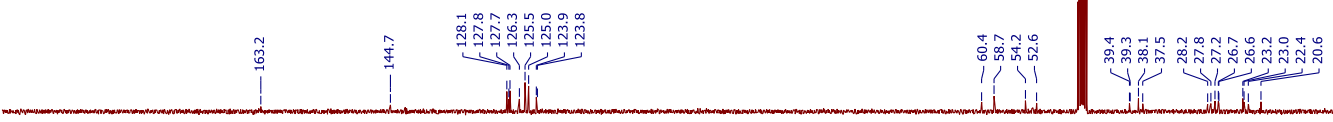

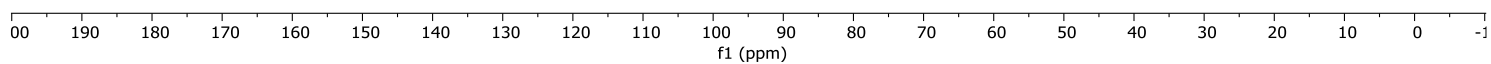


${ }^{1} \mathrm{H}$ NMR $\left(500 \mathrm{MHz}, \mathrm{CDCl}_{3}\right)$ of $\mathbf{6 s}$<smiles>CCOC(=O)CCC(c1ccccc1)(c1ccccc1)C1C=CCC1</smiles>

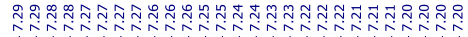

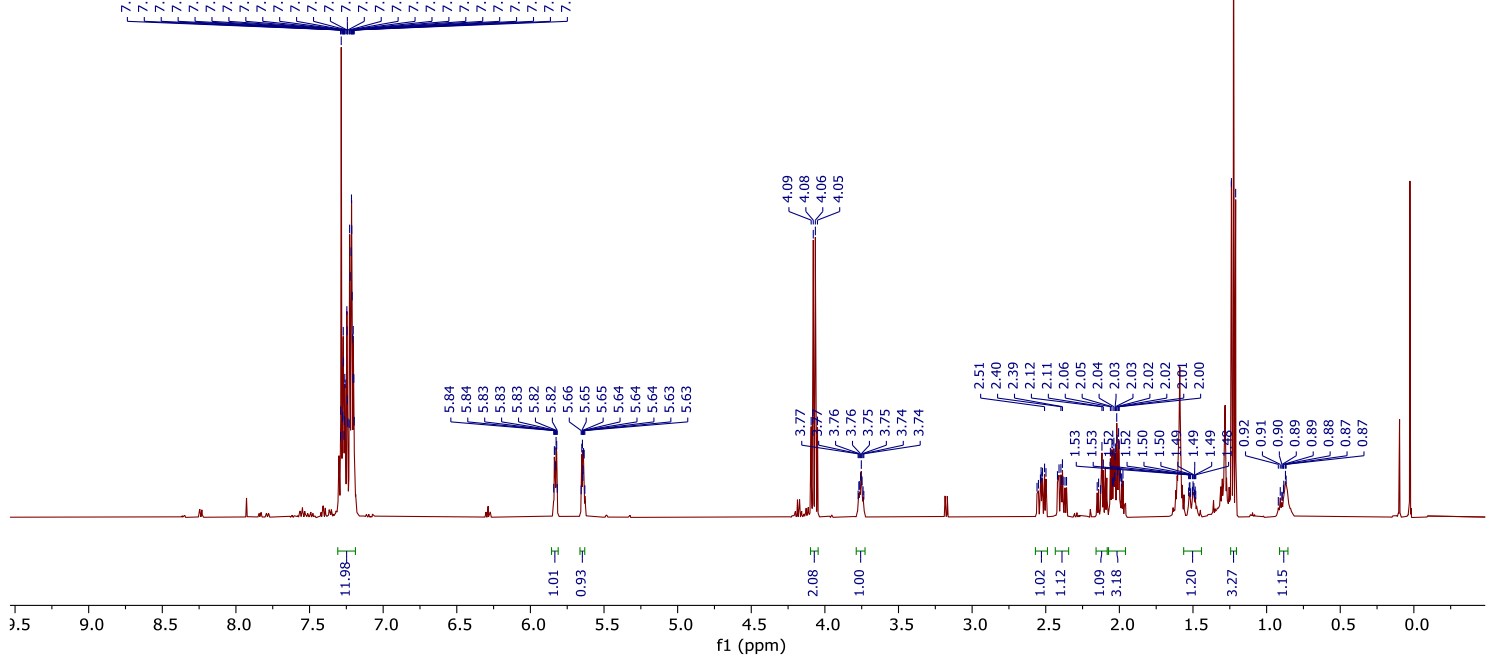

${ }^{13} \mathrm{C}$ NMR $\left(126 \mathrm{MHz}, \mathrm{CDCl}_{3}\right)$ of $\mathbf{6 s}$<smiles>CCOC(=O)CCC(c1ccccc1)(c1ccccc1)C1C=CCC1</smiles>

$6 s$

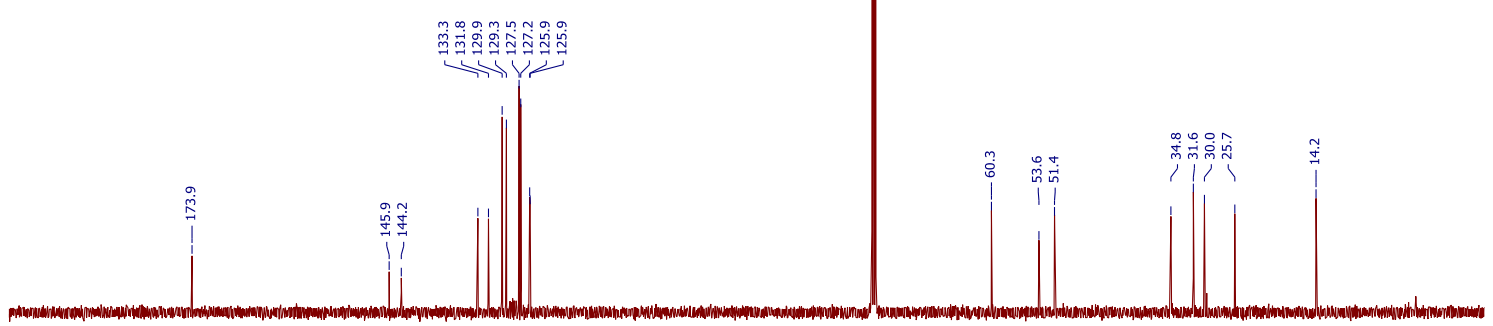


${ }^{1} \mathrm{H}$ NMR (400 MHz, $\left.\mathrm{CDCl}_{3}\right)$ of $\mathbf{6 t}$

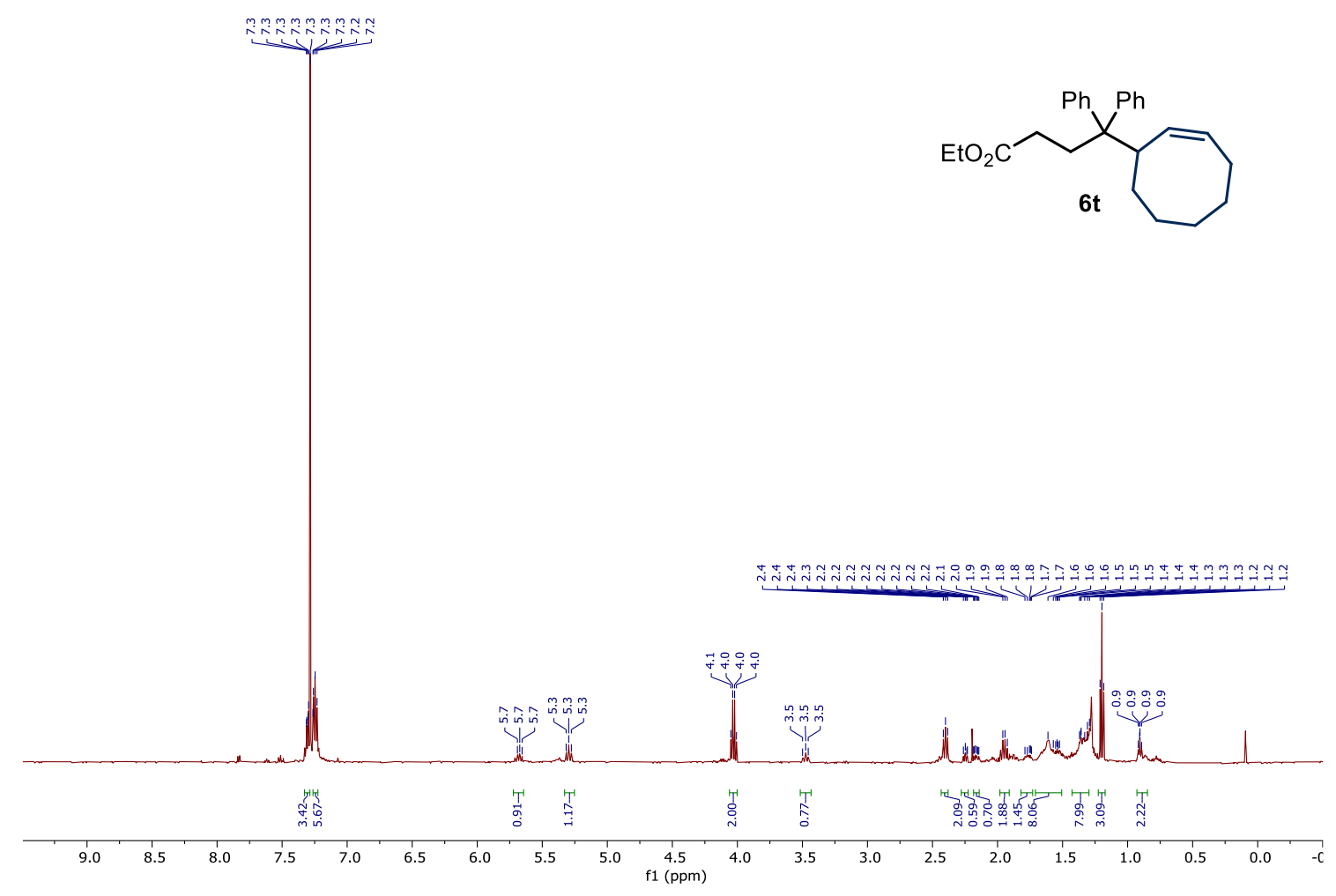

${ }^{13} \mathrm{C}$ NMR (101 MHz, $\left.\mathrm{CDCl}_{3}\right)$ of $\mathbf{6 t}$
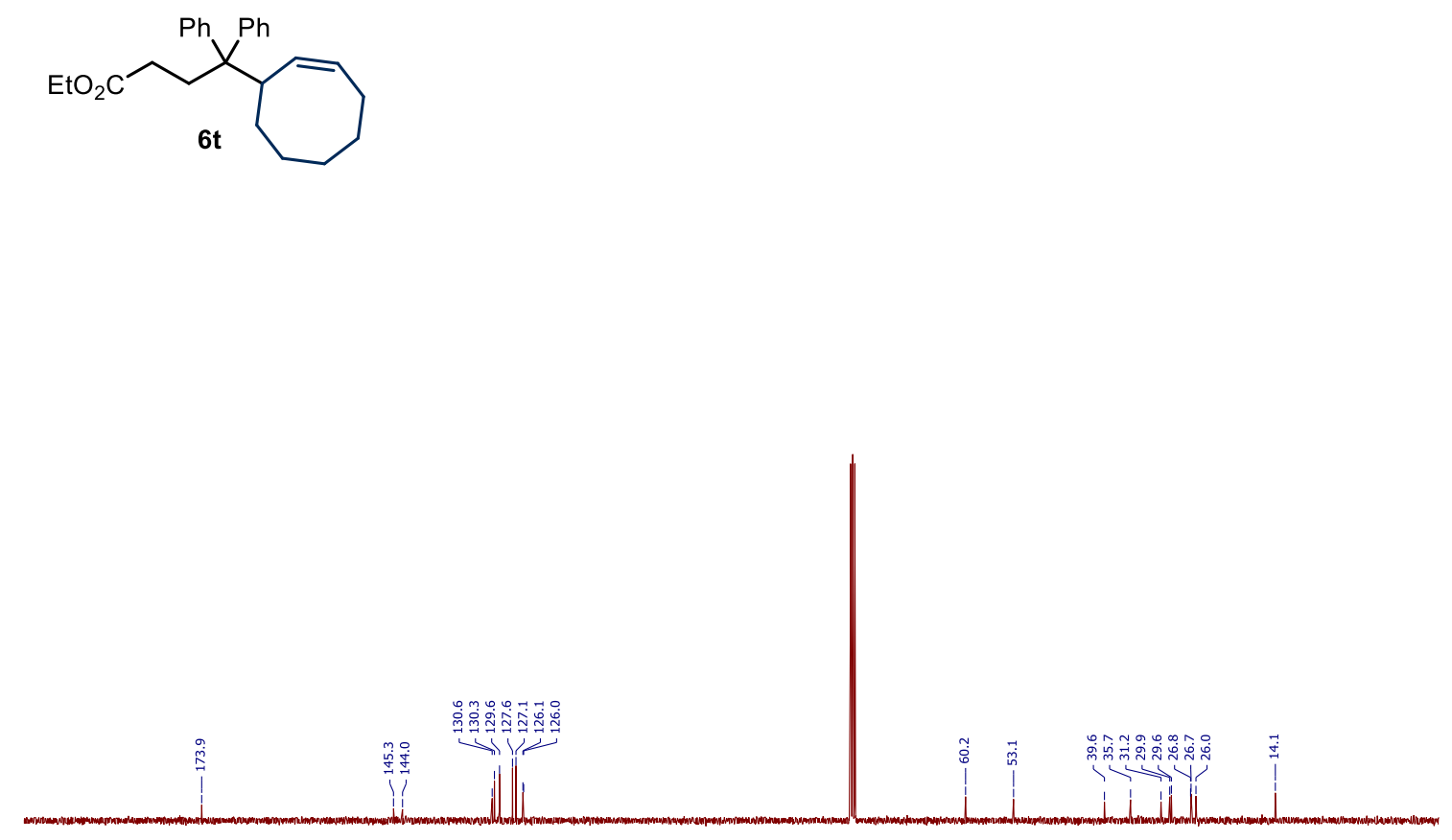

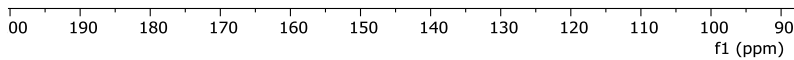


${ }^{1} \mathrm{H}$ NMR (400 MHz, $\left.\mathrm{CDCl}_{3}\right)$ of $\mathbf{6 u}$<smiles>CCOC(=O)CCCC(c1ccccc1)(c1ccccc1)C1C=C(C)CCC1</smiles>

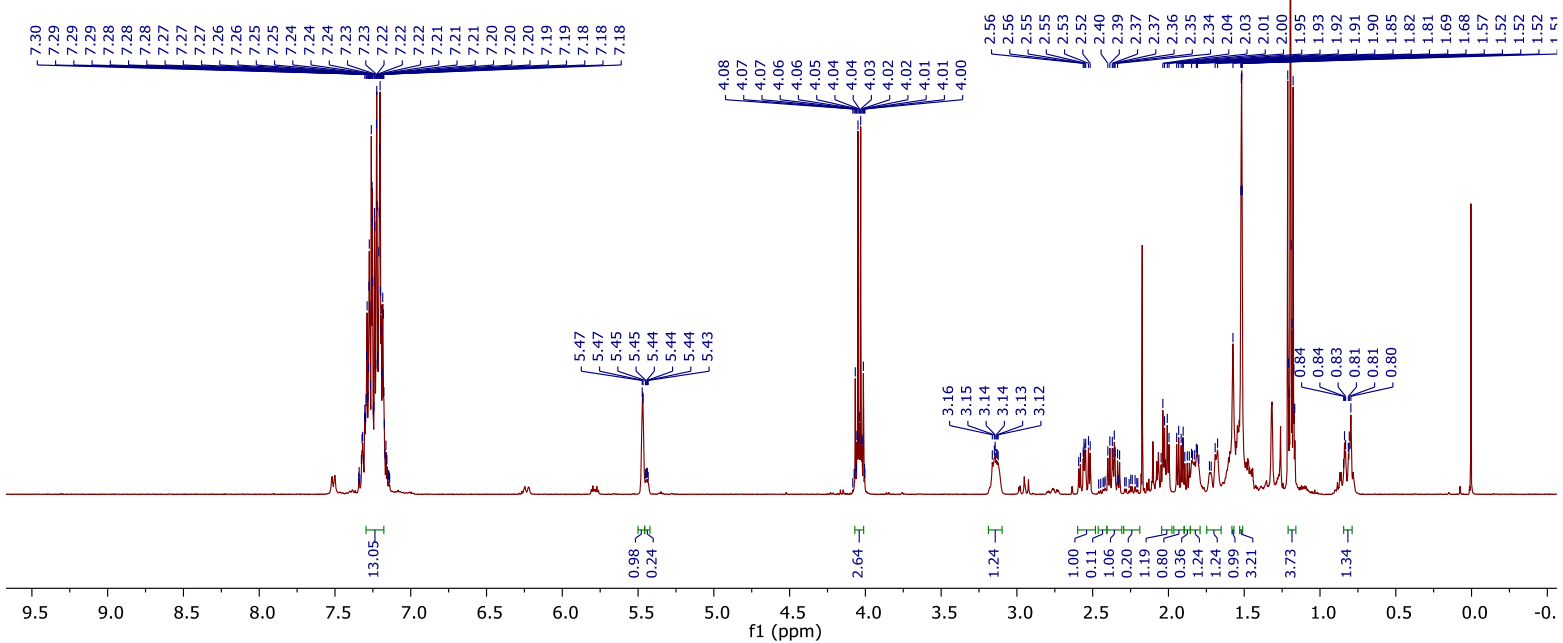

${ }^{13} \mathrm{C}$ NMR $\left(101 \mathrm{MHz}, \mathrm{CDCl}_{3}\right)$ of $\mathbf{6 u}$<smiles>CCOC(=O)CCCC(c1ccccc1)(c1ccccc1)C1C=C(C)CCC1</smiles>

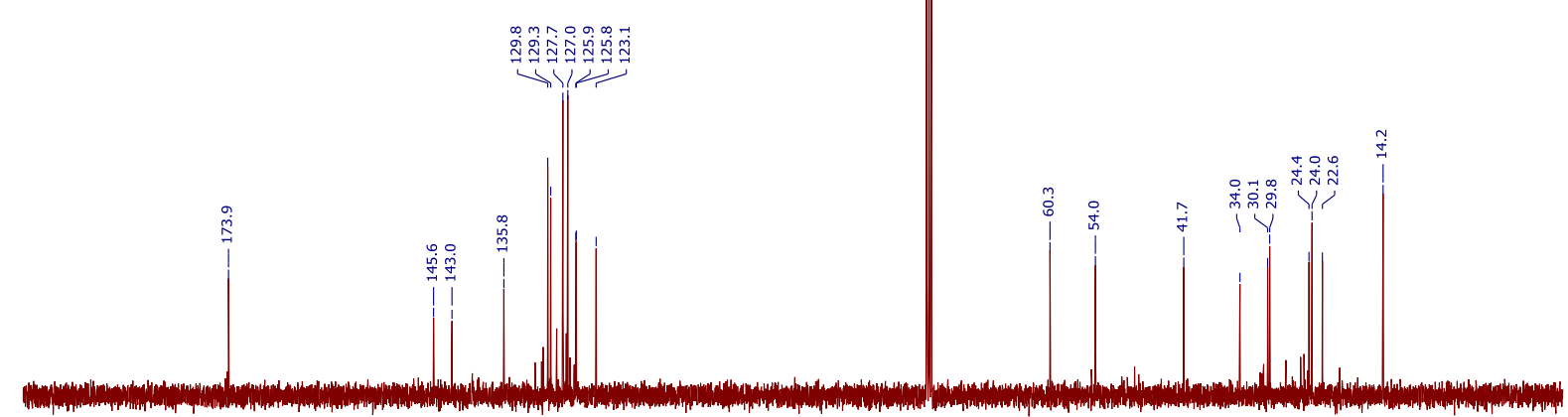

$\begin{array}{lllllllllll}200 & 190 & 180 & 170 & 160 & 150 & 140 & 130 & 120 & 110 & 100 \\ \mathrm{f} 1(\mathrm{ppm}) & 90\end{array}$ 
${ }^{1} \mathrm{H}$ NMR (400 MHz, $\mathrm{CDCl}_{3}$ ) of $\mathbf{6 v}$

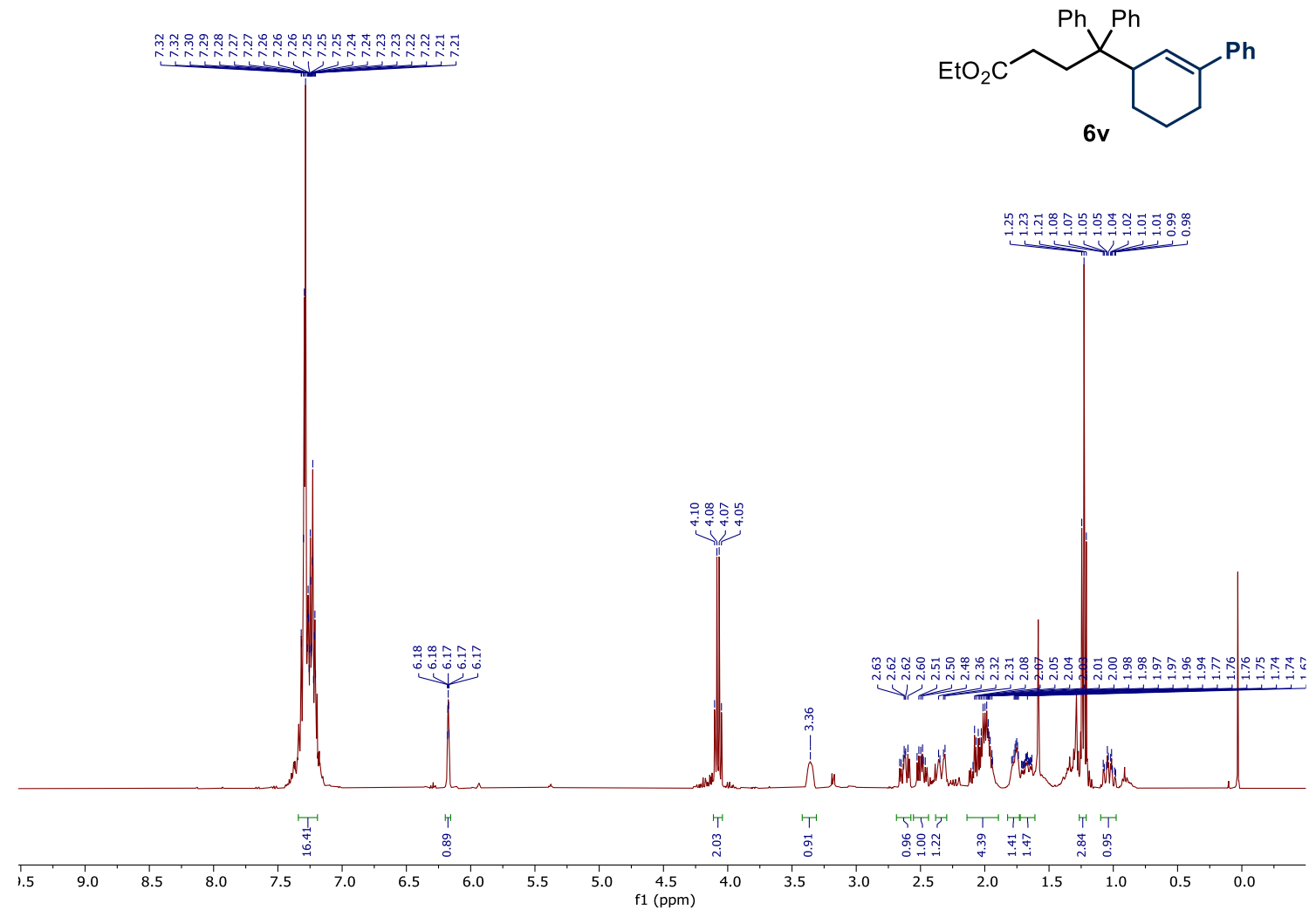

${ }^{13} \mathrm{C}$ NMR (101 MHz, $\left.\mathrm{CDCl}_{3}\right)$ of $\mathbf{6 v}$

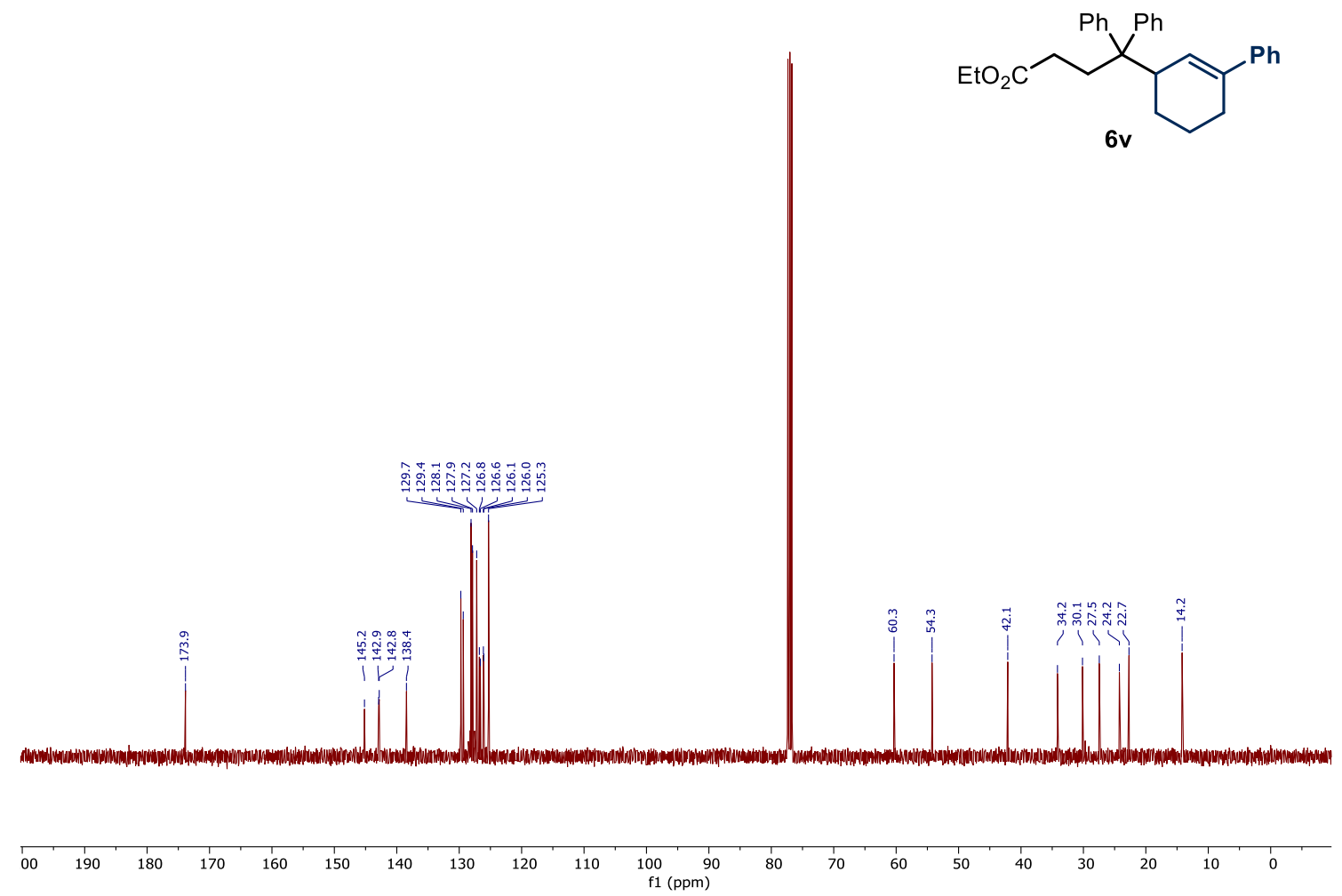


${ }^{1} \mathrm{H}$ NMR $\left(500 \mathrm{MHz}, \mathrm{CDCl}_{3}\right)$ of $\mathbf{6 w}$

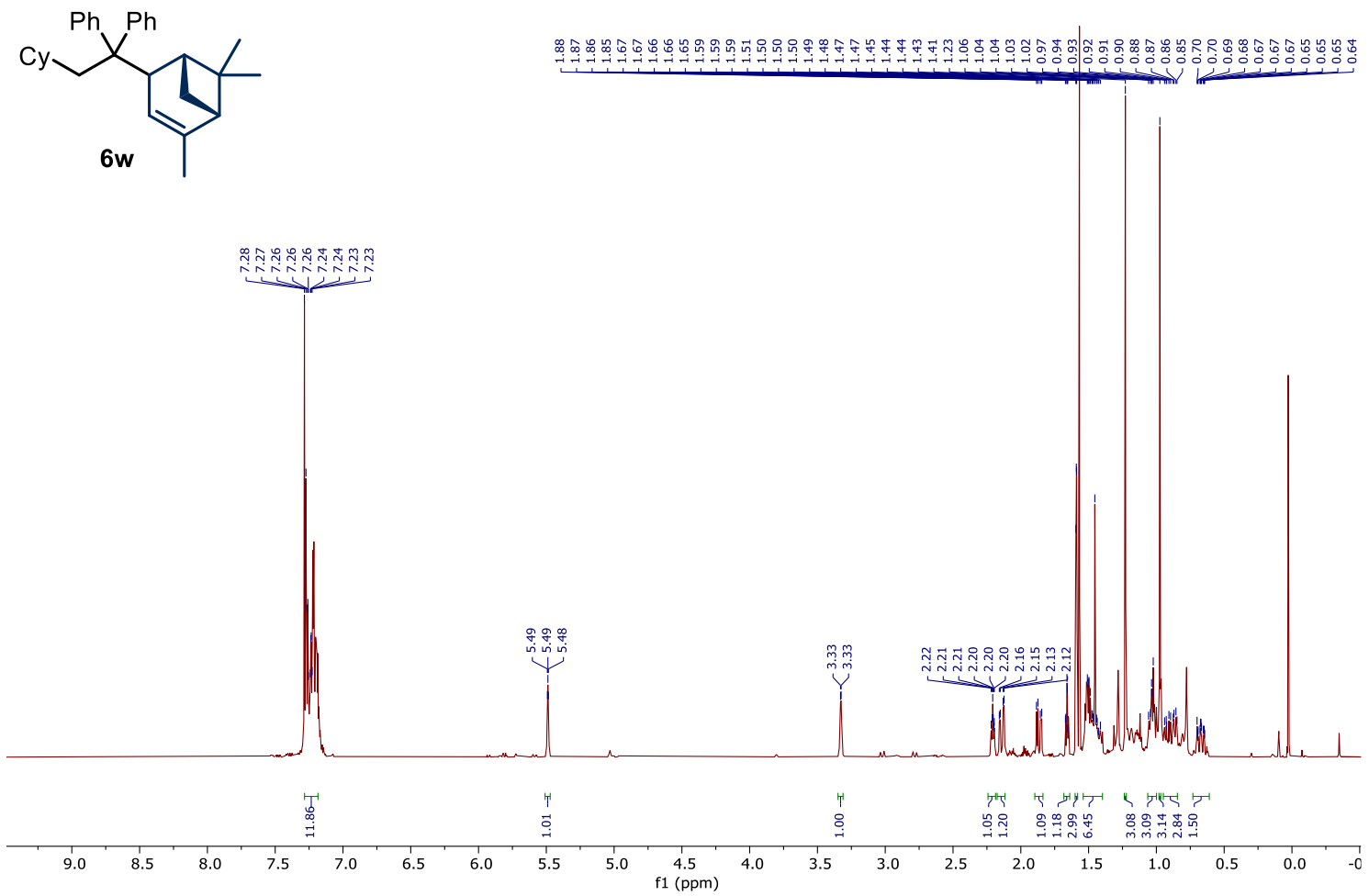

${ }^{13} \mathrm{C}$ NMR (101 MHz, $\left.\mathrm{CDCl}_{3}\right)$ of $\mathbf{6 w}$
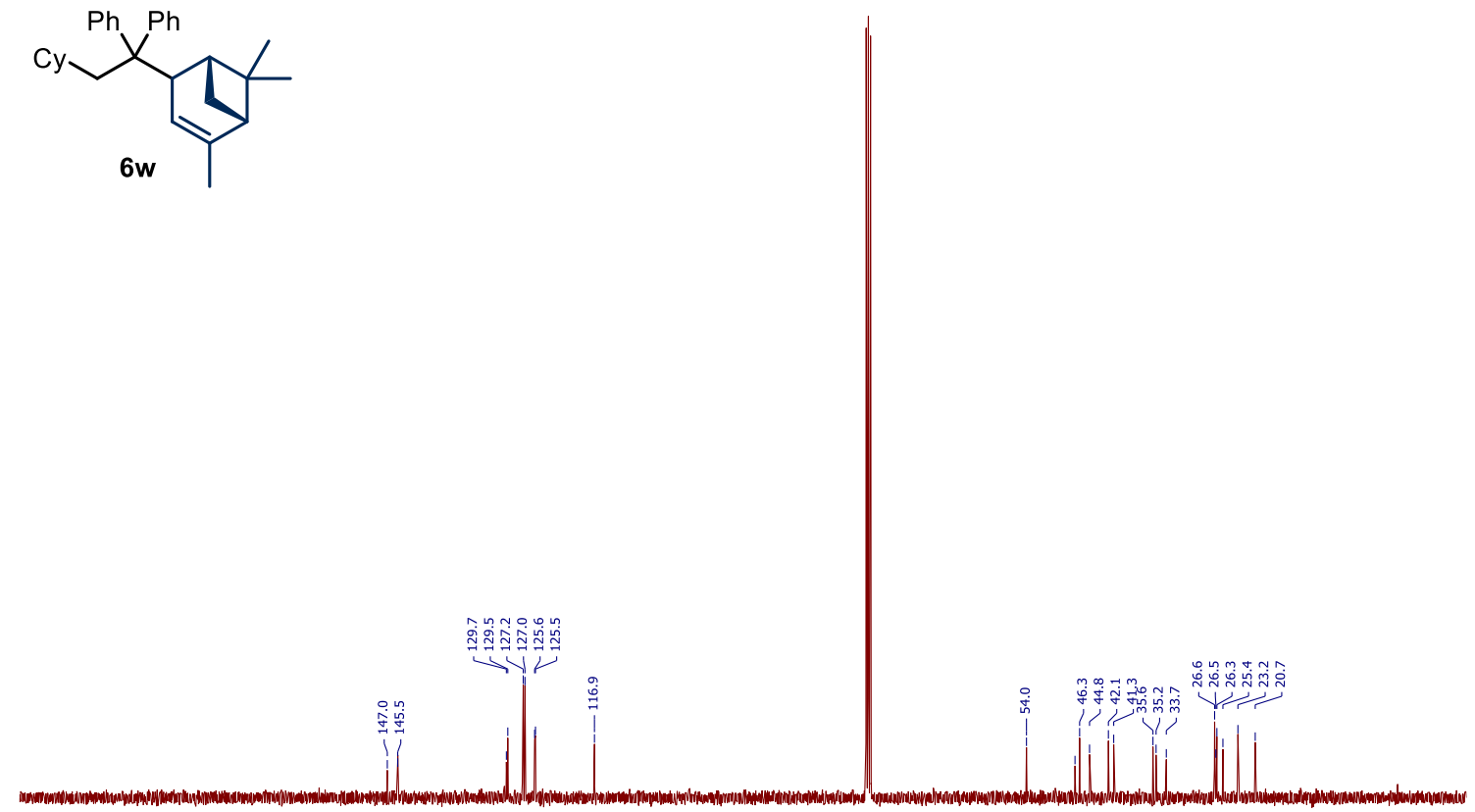

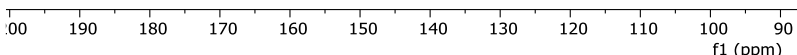


${ }^{1} \mathrm{H} \mathrm{NMR}\left(\mathbf{5 0 0} \mathrm{MHz}, \mathrm{CDCl}_{3}\right)$ of $\mathbf{6 x}$<smiles>CCOC(=O)CCCC(c1ccccc1)(c1ccccc1)C1C=C(CCN=P)CCC1C(CCCNc1ccccc1)(c1ccccc1)c1ccccc1</smiles>
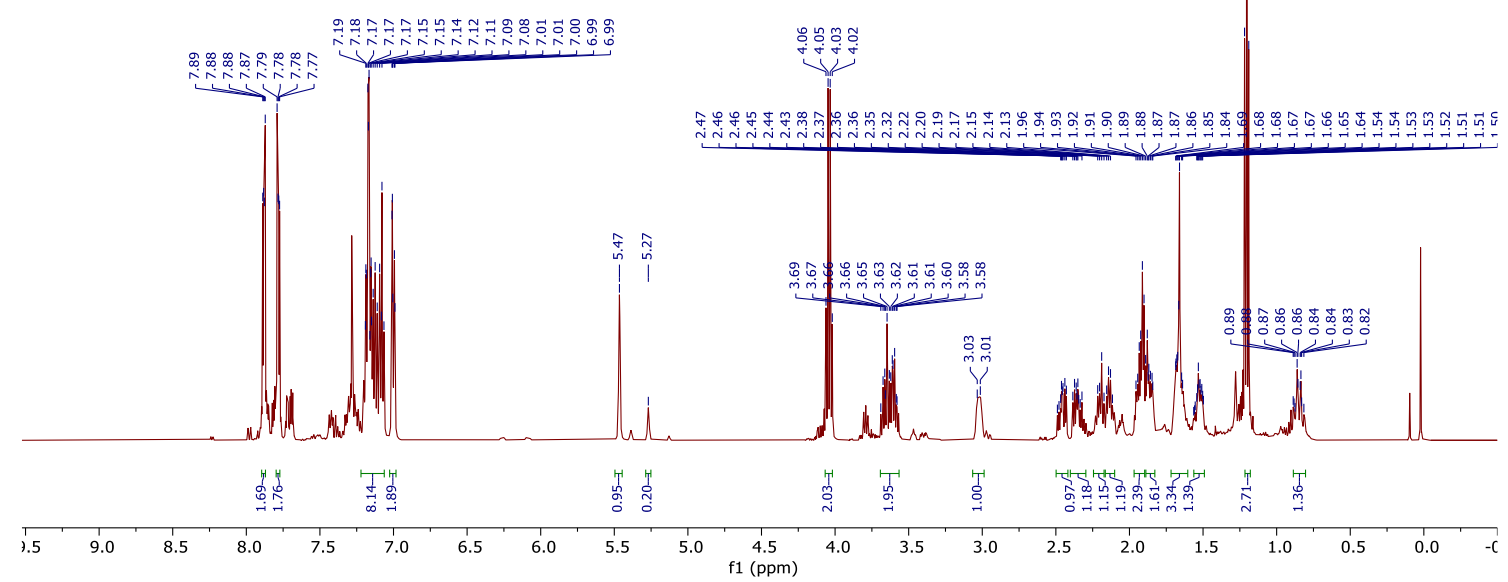

${ }^{13} \mathrm{C}$ NMR $\left(126 \mathrm{MHz}, \mathrm{CDCl}_{3}\right)$ of $\mathbf{6} \mathbf{x}$<smiles>CCOC(=O)CCCC(c1ccccc1)(c1ccccc1)C1CCCC=C1CCN=Pc1ccccc1</smiles>

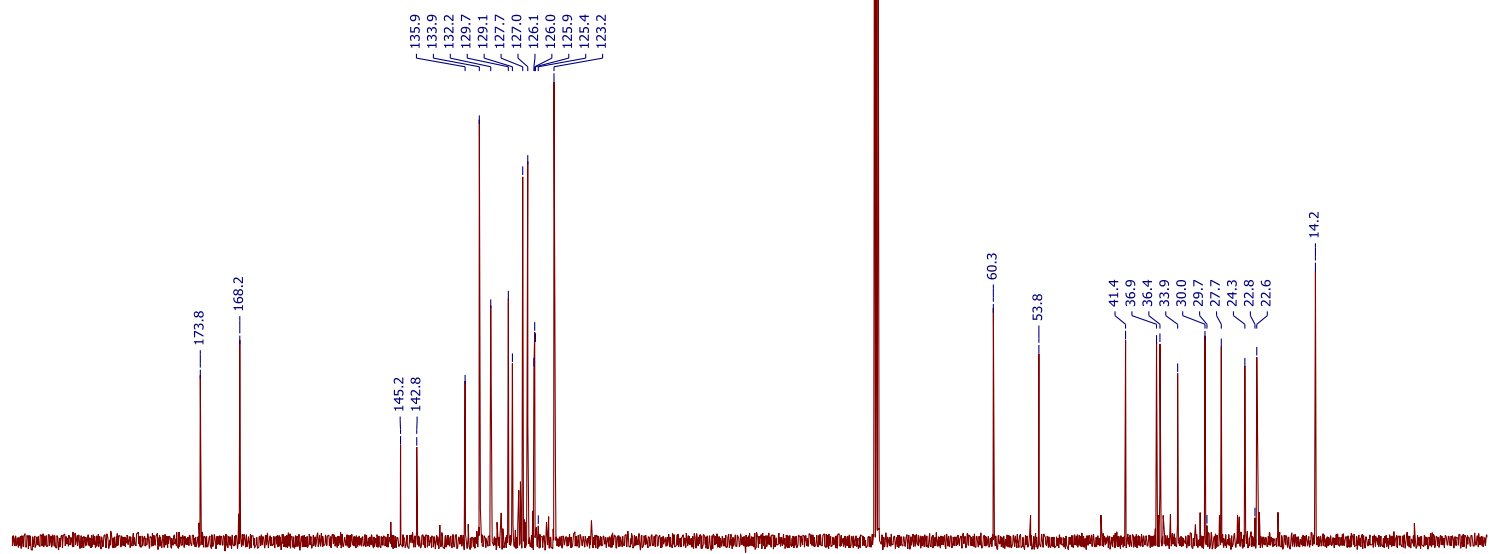

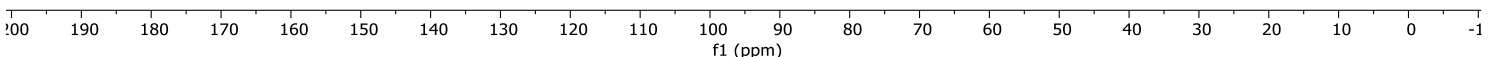


${ }^{1} \mathrm{H}$ NMR $\left(500 \mathrm{MHz}, \mathrm{CDCl}_{3}\right)$ of $\mathbf{6 y}$<smiles>CCOC(=O)CCCC(c1ccccc1)(c1ccccc1)C1C=C(Cc2ccccc2)CCC1</smiles>

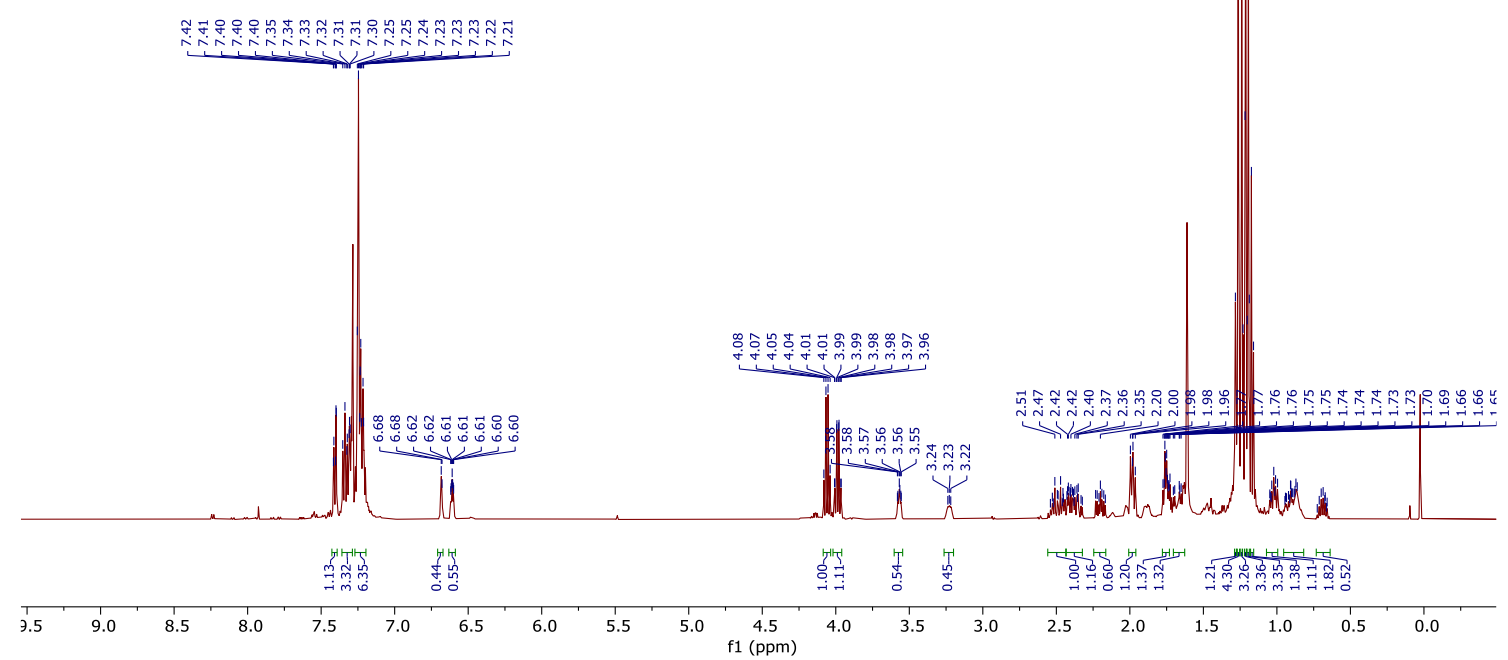

${ }^{13} \mathrm{C}$ NMR $\left(101 \mathrm{MHz}, \mathrm{CDCl}_{3}\right)$ of $\mathbf{6 y}$<smiles>CCOC(=O)CCCC(c1ccccc1)(c1ccccc1)C1C=C(Cc2ccccc2)CCC1</smiles>

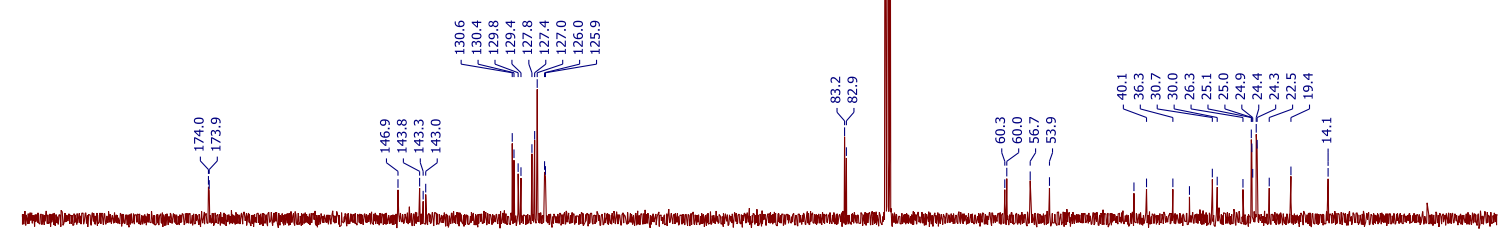

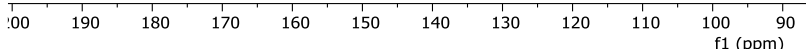


${ }^{1} \mathrm{H} \mathrm{NMR}\left(\mathbf{5 0 0} \mathrm{MHz}, \mathrm{CDCl}_{3}\right)$ of $\mathbf{6 z}$

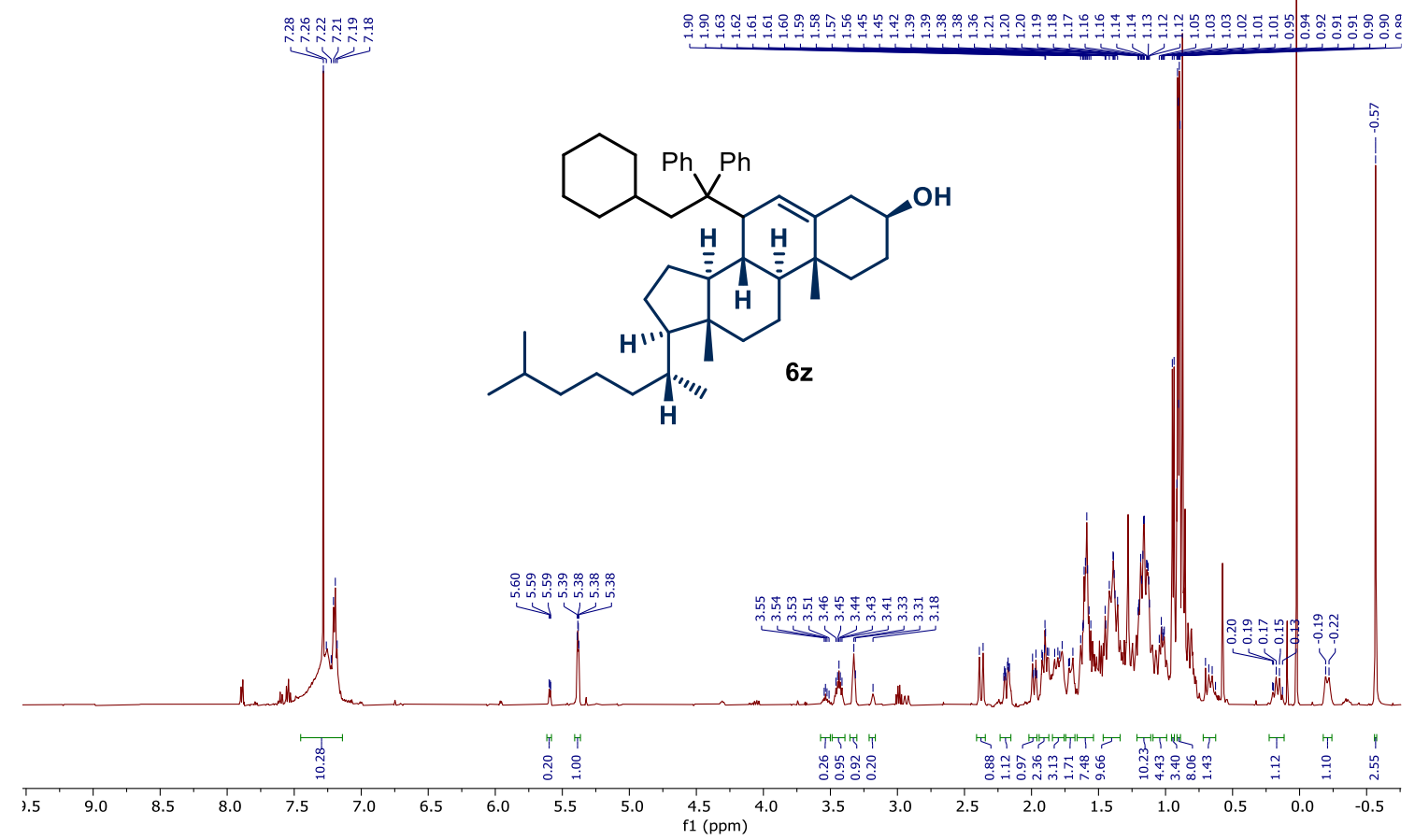

${ }^{13} \mathrm{C}$ NMR $\left(126 \mathrm{MHz}, \mathrm{CDCl}_{3}\right)$ of $\mathbf{6 z}$

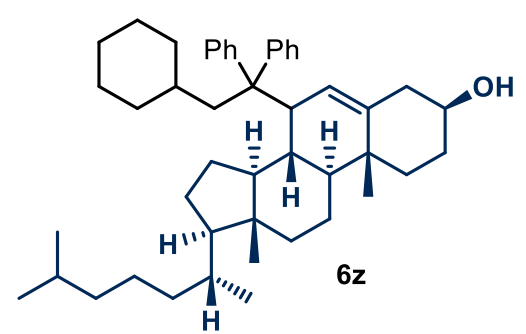

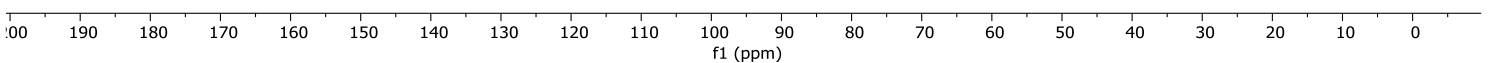


${ }^{1} \mathrm{H}$ NMR $\left(\mathbf{5 0 0 ~ M H z}, \mathrm{CDCl}_{3}\right)$ of $\mathbf{6 a a}$

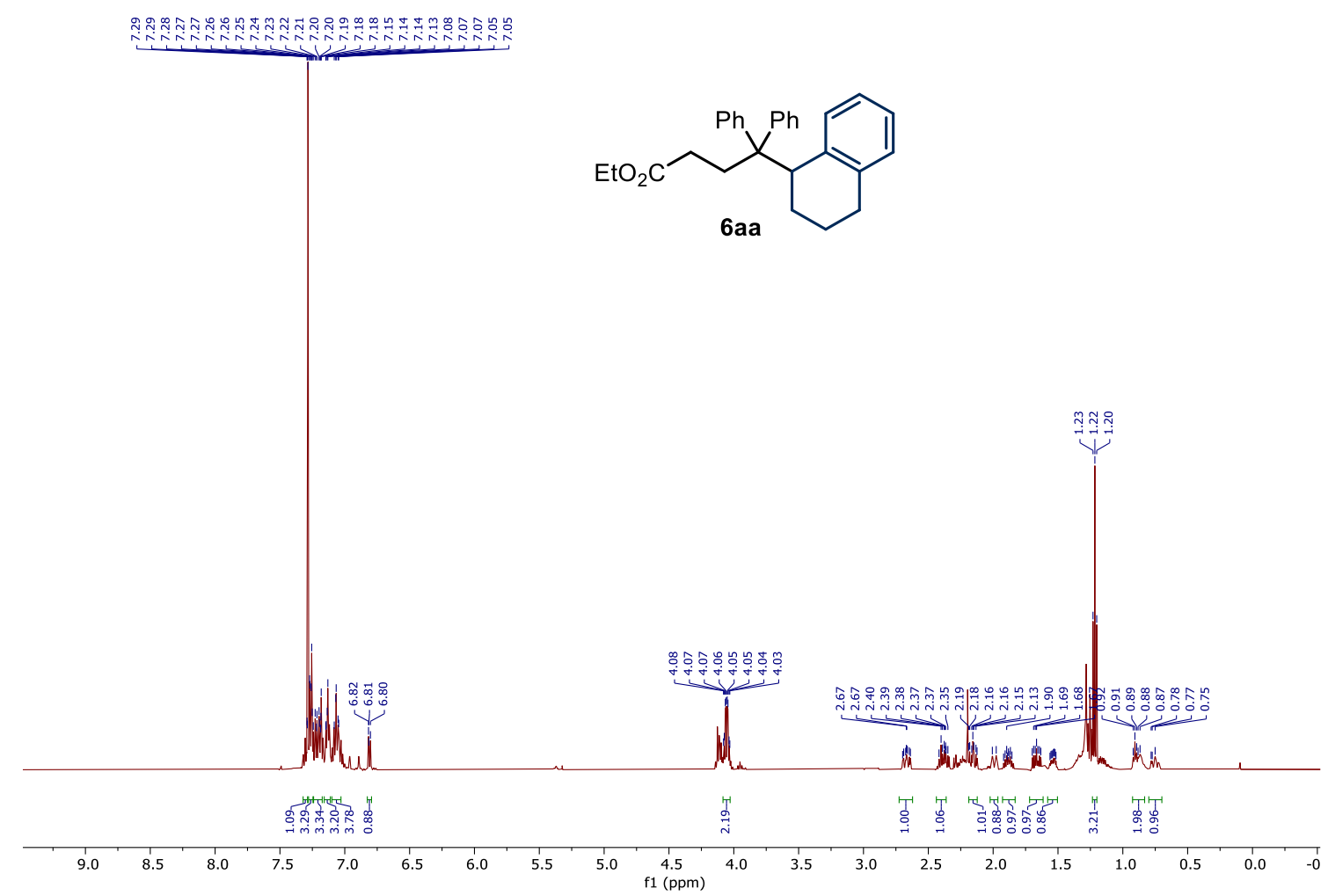

${ }^{13} \mathrm{C}$ NMR $\left(126 \mathrm{MHz}, \mathrm{CDCl}_{3}\right)$ of $\mathbf{6 a a}$<smiles>CCOC(=O)CCC(c1ccccc1)(c1ccccc1)C1CCCc2ccccc21</smiles>

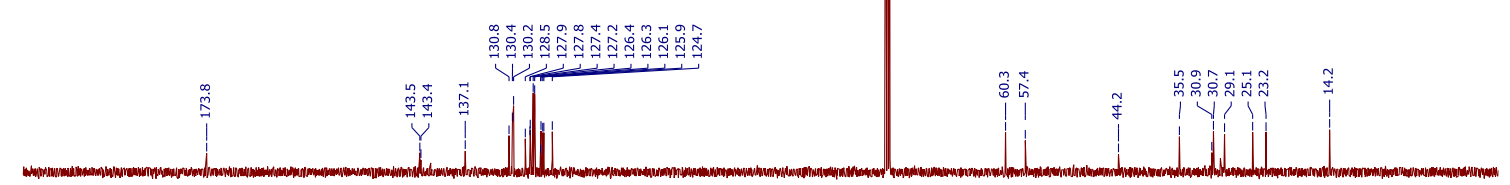

$\begin{array}{llllllllll}190 & 180 & 170 & 160 & 150 & 140 & 130 & 120 & 110 & \begin{array}{r}100 \\ \text { f1 (ppm) }\end{array}\end{array}$ 
${ }^{1} \mathrm{H}$ NMR $\left(400 \mathrm{MHz}, \mathrm{CDCl}_{3}\right)$ of $\mathbf{6 b b}$
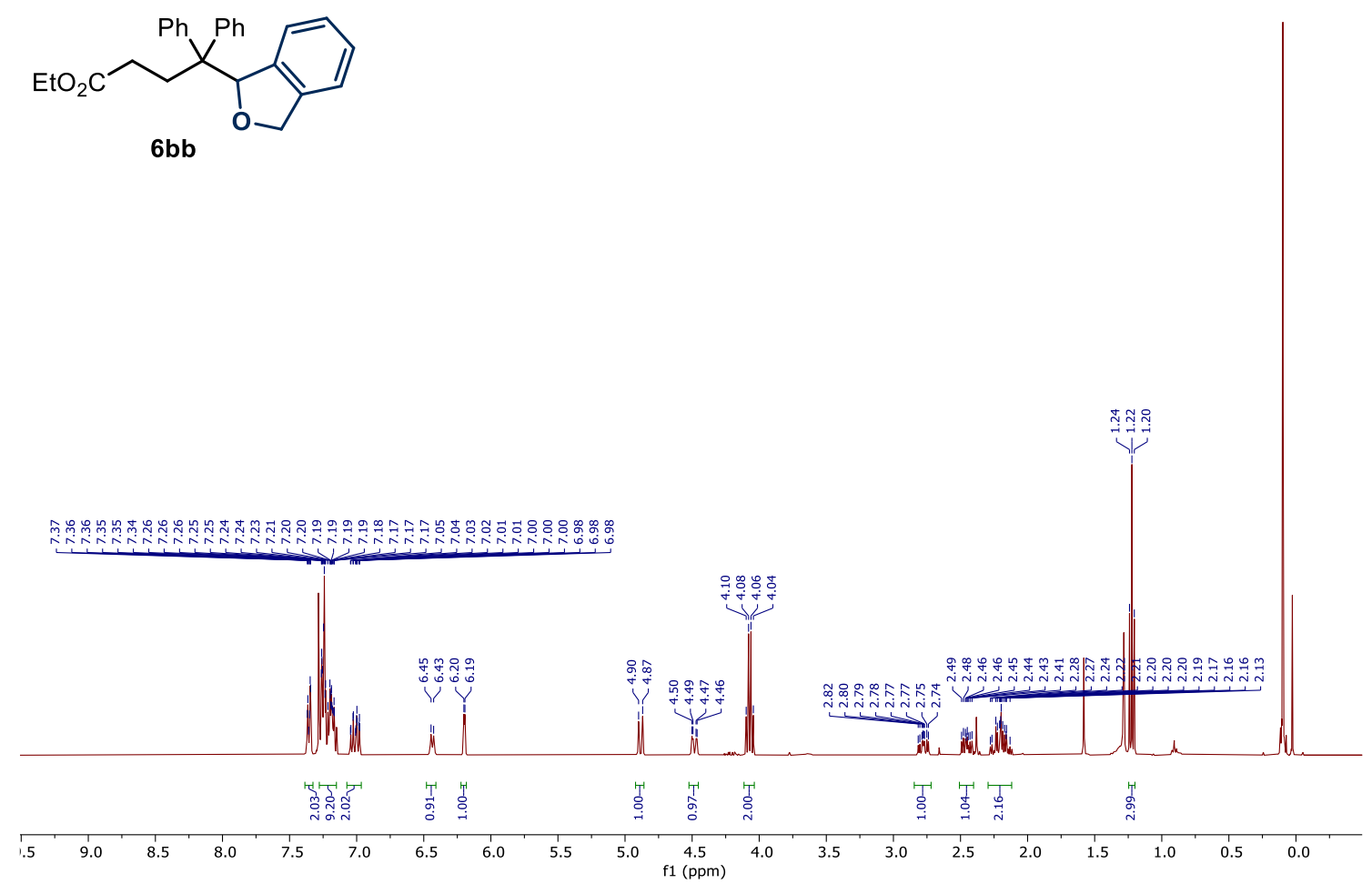

${ }^{13} \mathrm{C}$ NMR (101 MHz, $\left.\mathrm{CDCl}_{3}\right)$ of $\mathbf{6 b b}$

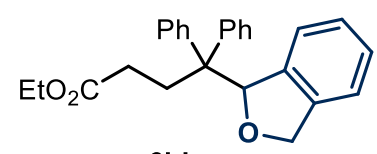

$6 b b$

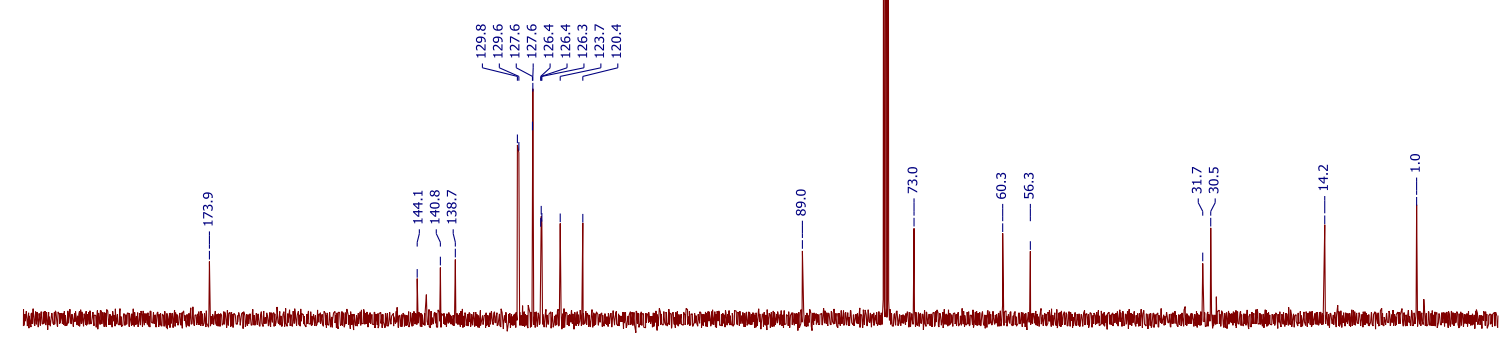

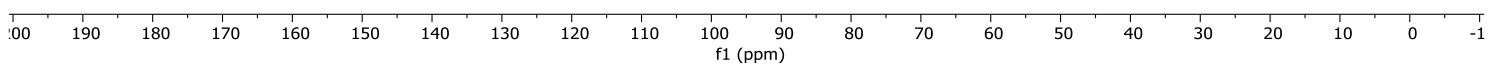


${ }^{1} \mathrm{H} \mathrm{NMR}\left(\mathbf{5 0 0} \mathrm{MHz}, \mathrm{CDCl}_{3}\right)$ of $\mathbf{6 c c}$

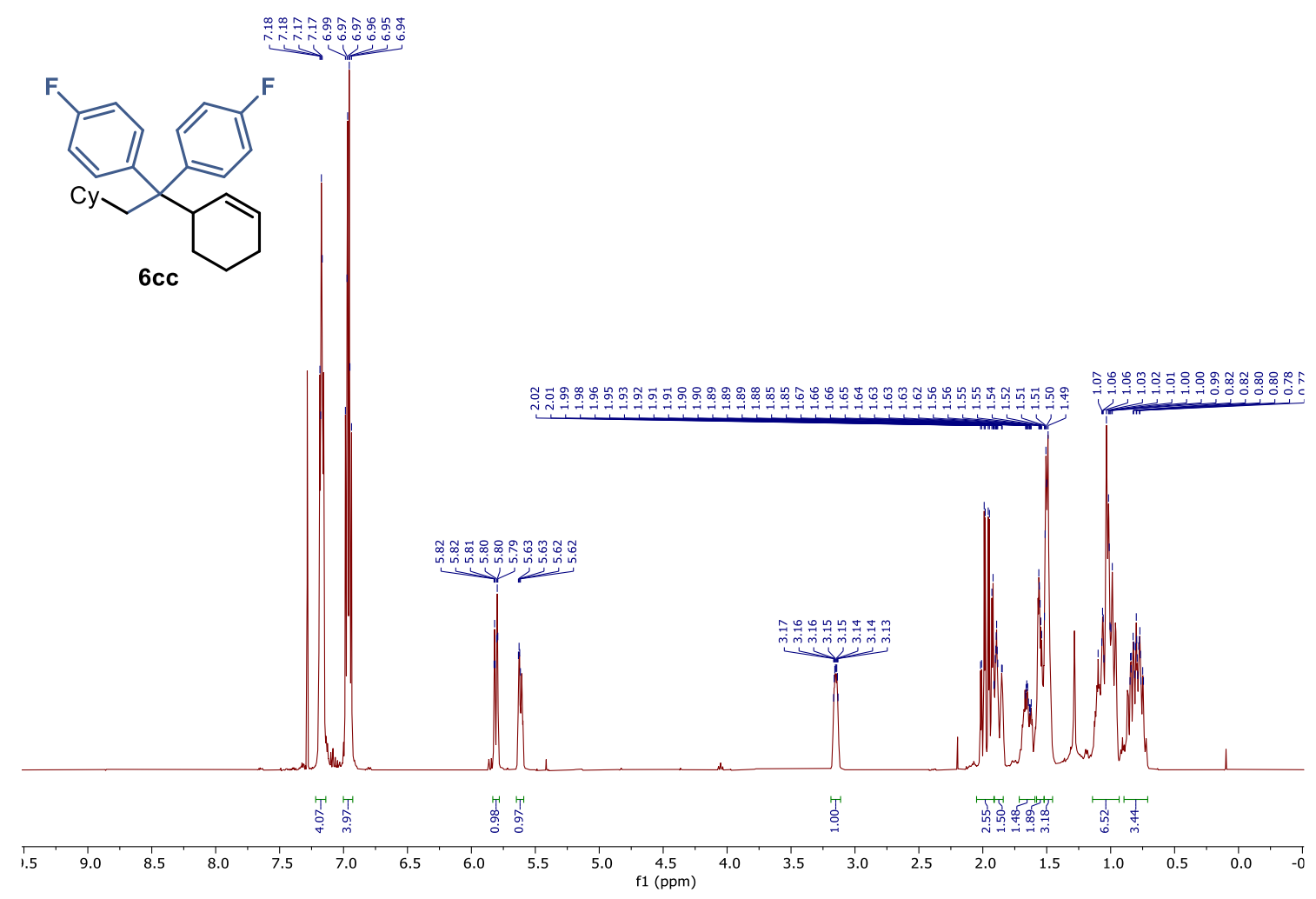

${ }^{13} \mathrm{C}$ NMR $\left(126 \mathrm{MHz}, \mathrm{CDCl}_{3}\right)$ of $\mathbf{6 c c}$
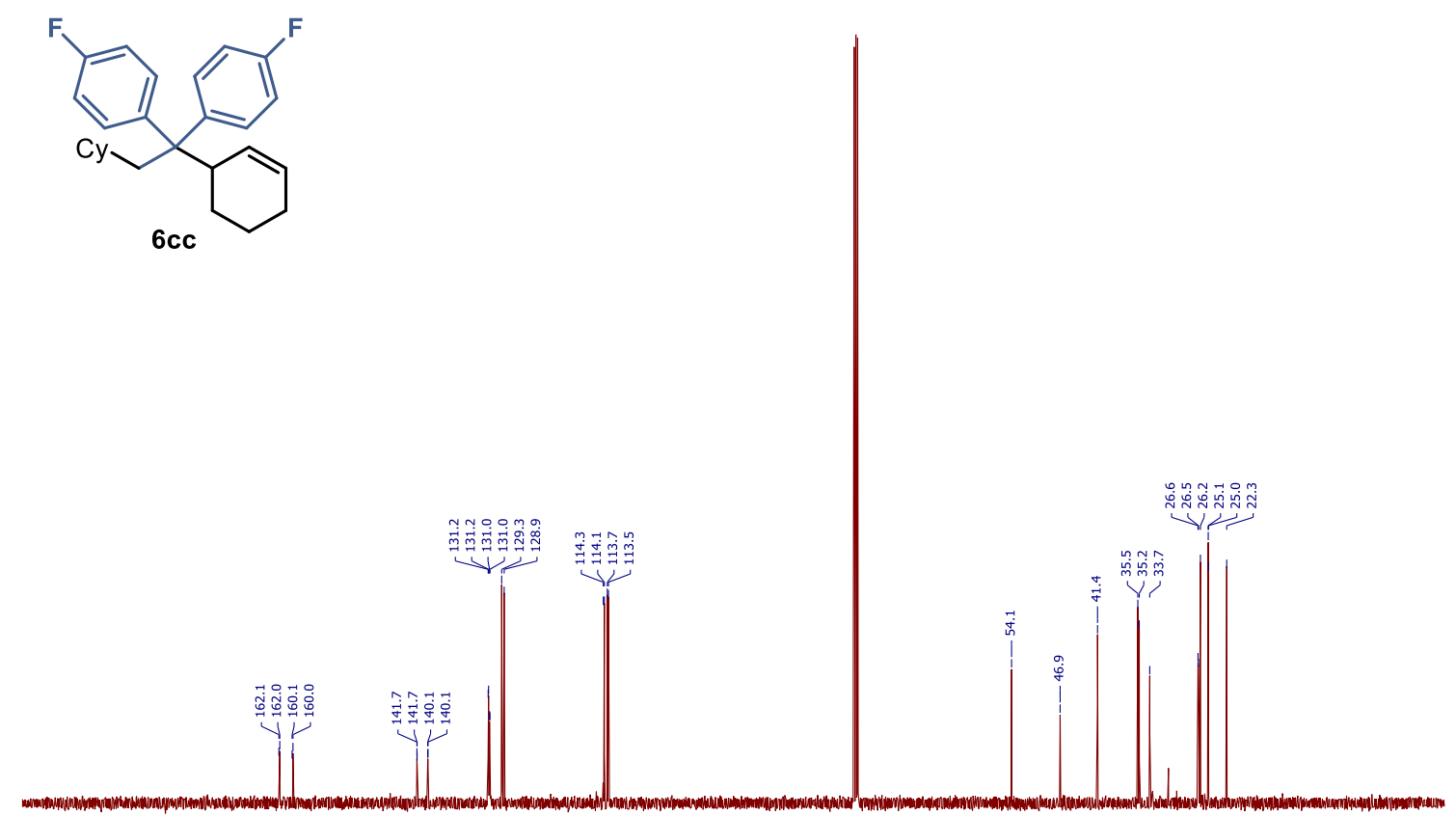

$\begin{array}{llllllllll}190 & 180 & 170 & 160 & 150 & 140 & 130 & 120 & 110 & \begin{array}{r}100 \\ \text { f1 (ppm) }\end{array}\end{array}$ 
${ }^{19} \mathrm{~F} \mathrm{NMR}\left(376 \mathrm{MHz}, \mathrm{CDCl}_{3}\right)$ of $\mathbf{6 c c}$
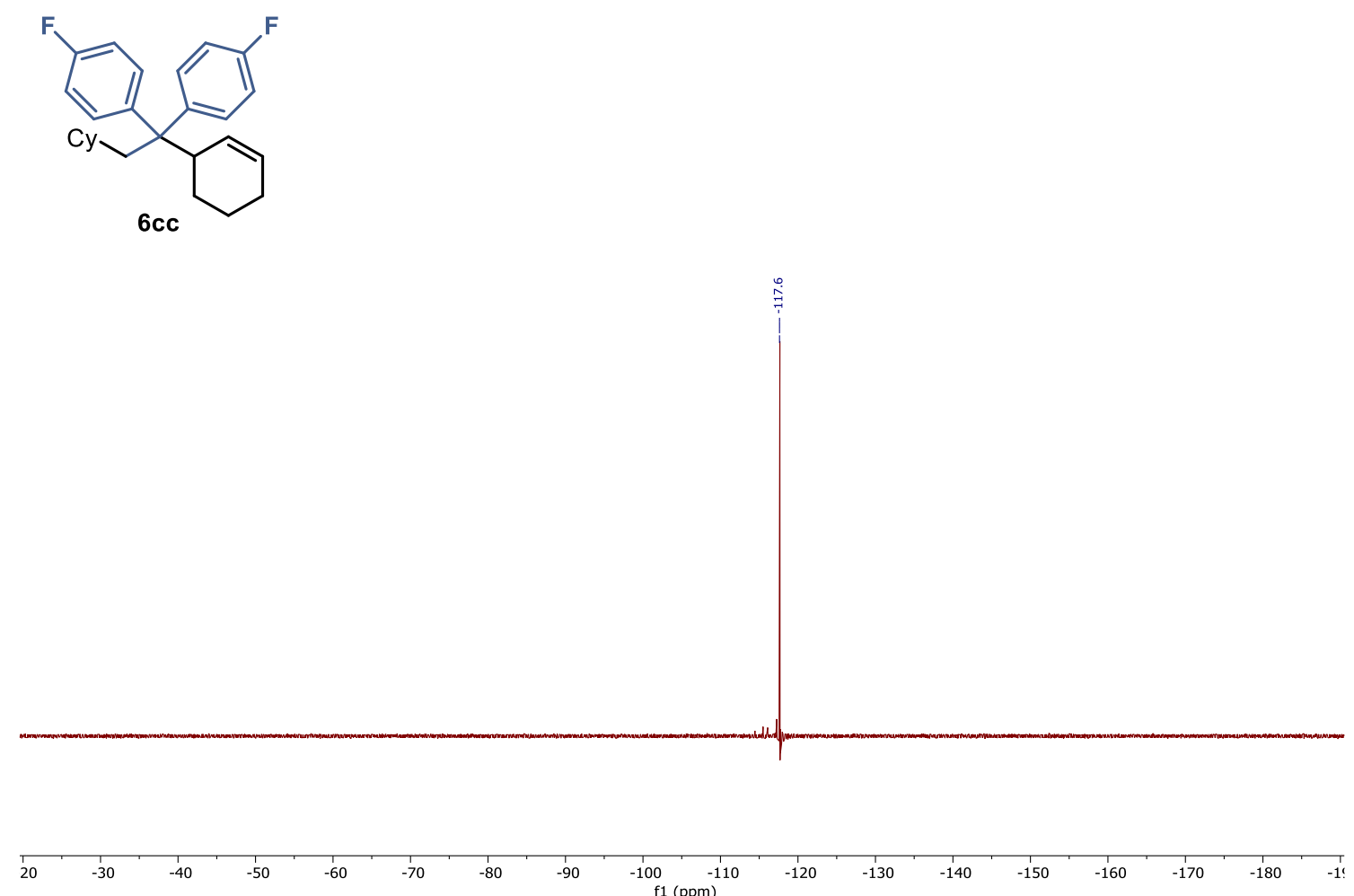


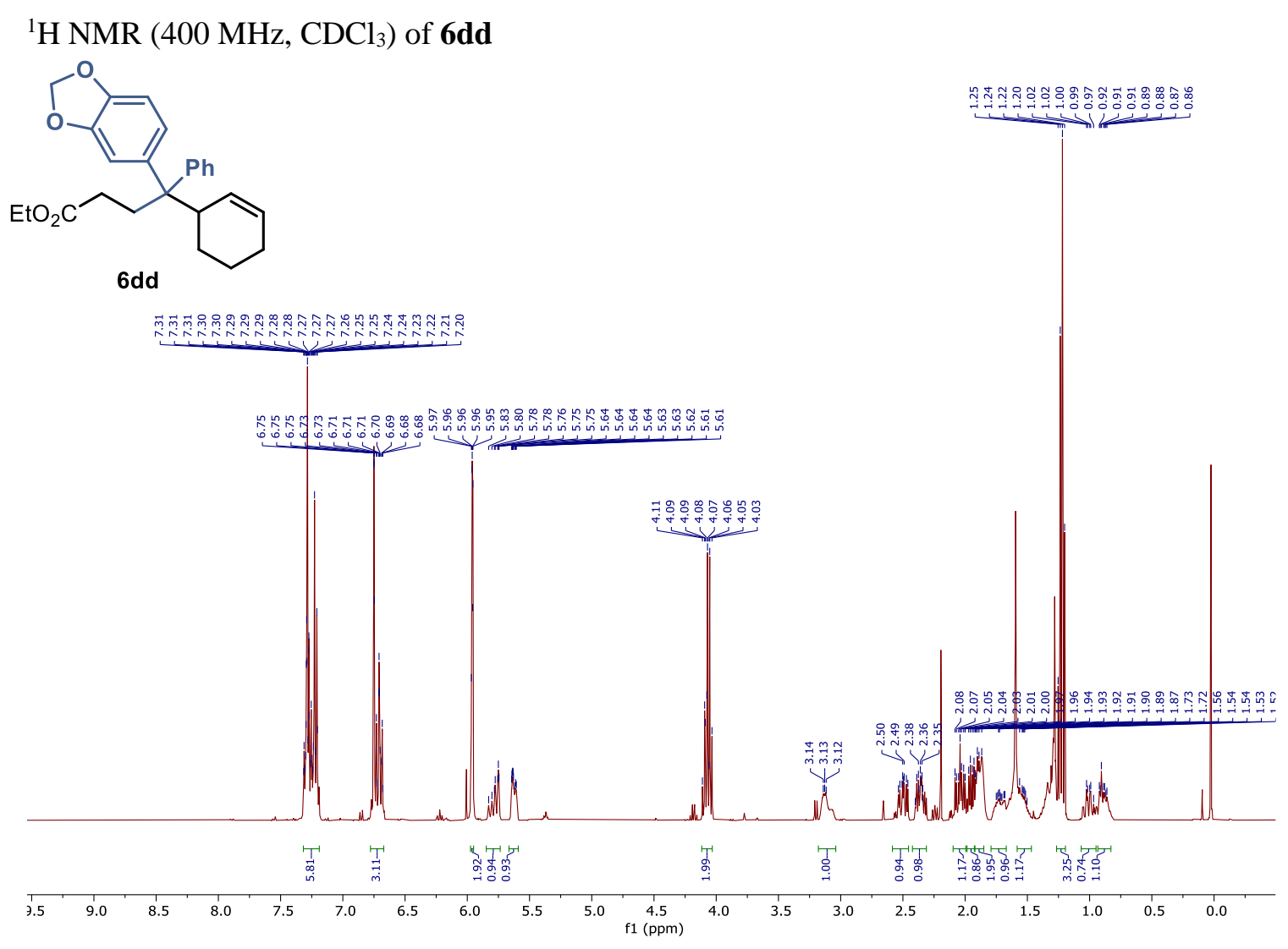

${ }^{13} \mathrm{C}$ NMR $\left(101 \mathrm{MHz}, \mathrm{CDCl}_{3}\right)$ of $\mathbf{6 d d}$
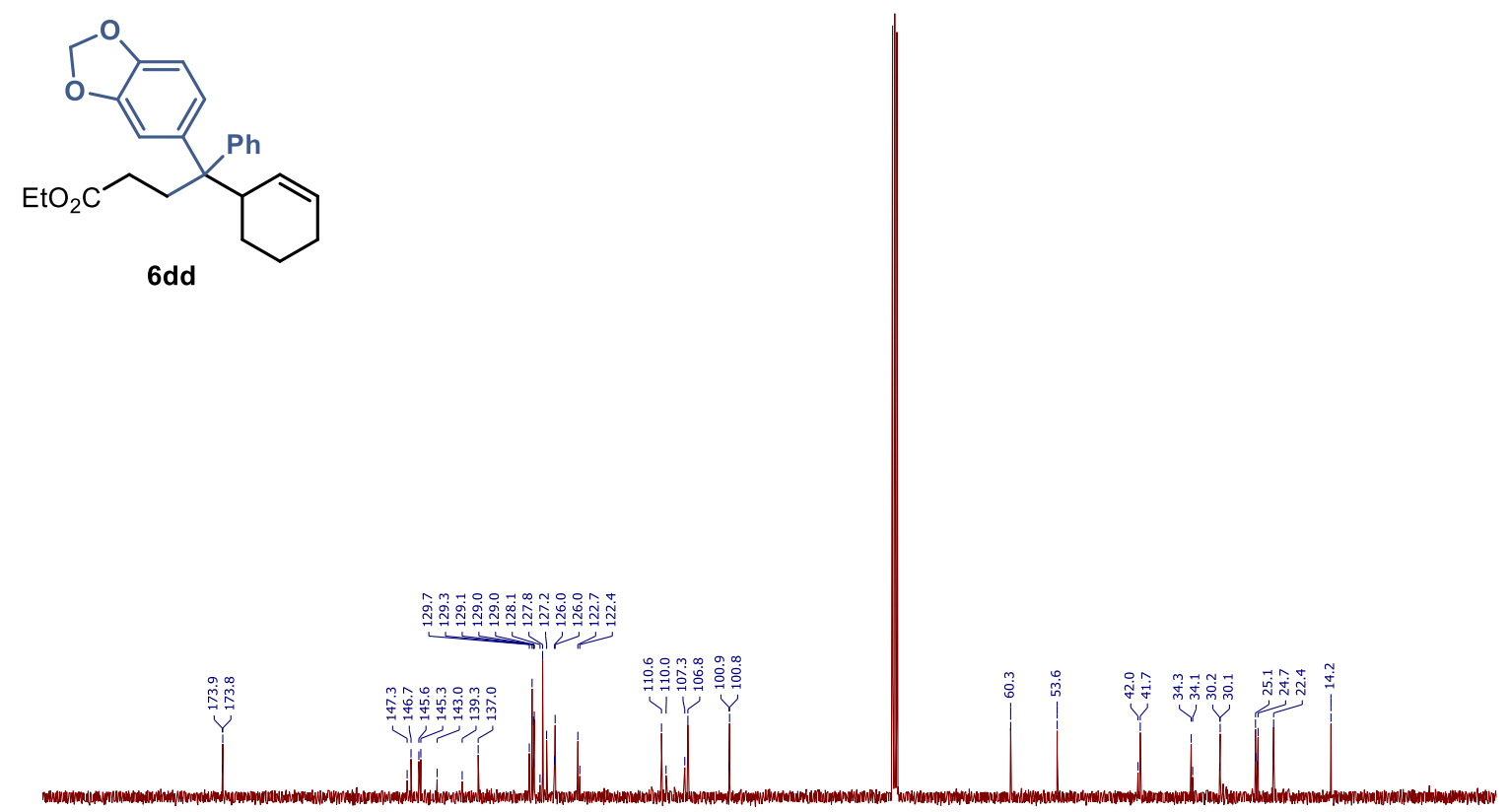
${ }^{1} \mathrm{H}$ NMR (400 MHz, $\mathrm{CDCl}_{3}$ ) of 6ee
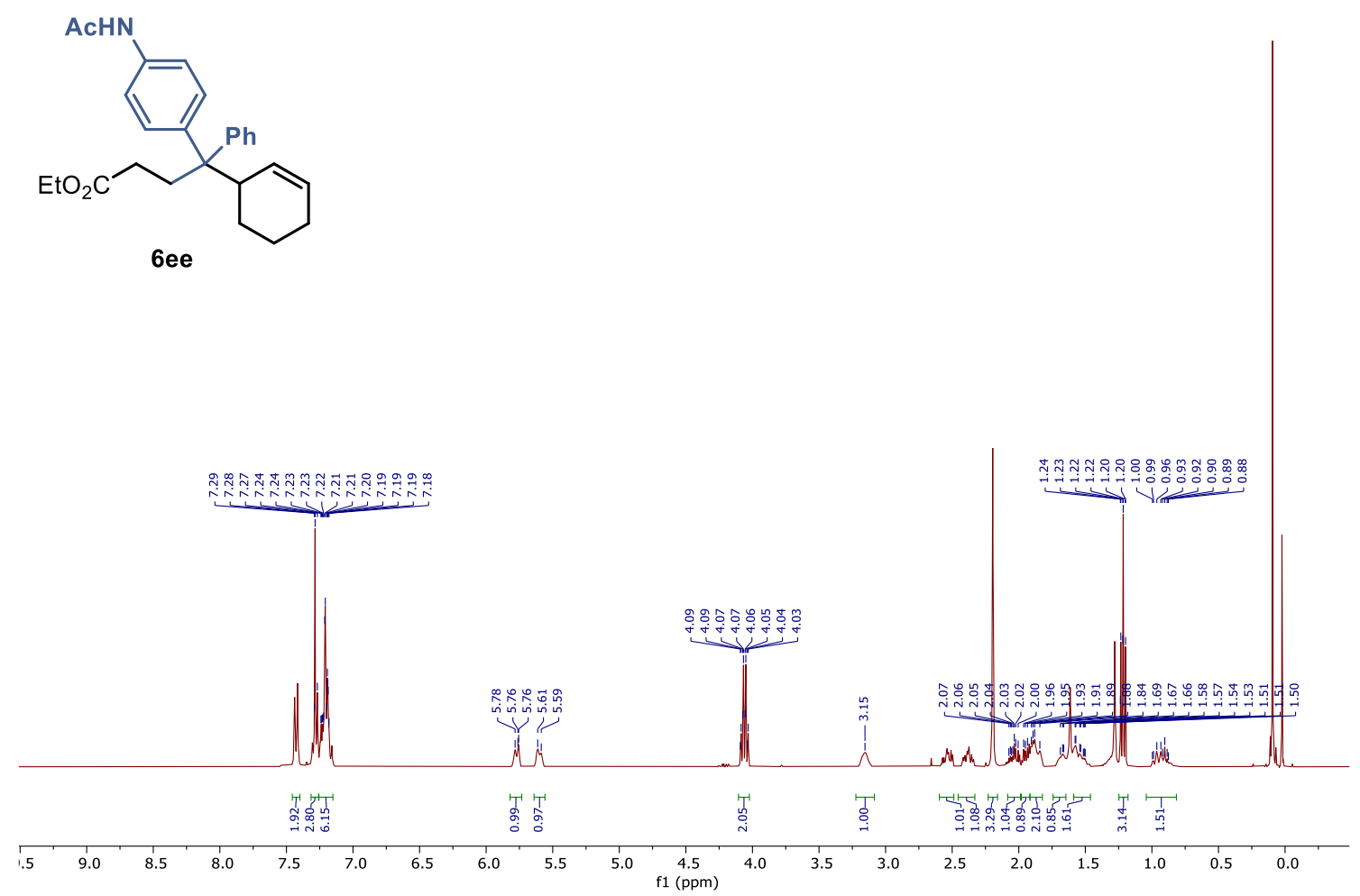

${ }^{13} \mathrm{C}$ NMR $\left(101 \mathrm{MHz}, \mathrm{CDCl}_{3}\right)$ of $\mathbf{6 e e}$

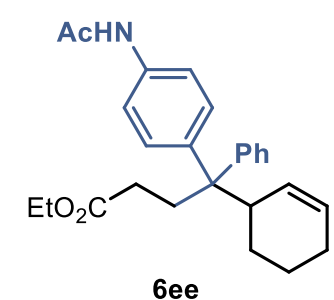

6ee

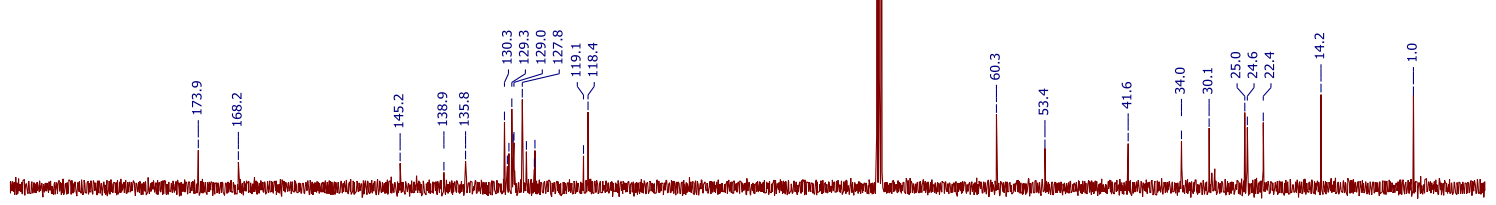

$\begin{array}{lllllllllll}100 & 190 & 180 & 170 & 160 & 150 & 140 & 130 & 120 & 110 & \begin{array}{c}100 \\ \mathrm{f} 1(\mathrm{ppm})\end{array}\end{array}$ 
${ }^{1} \mathrm{H}$ NMR $\left(500 \mathrm{MHz}, \mathrm{CDCl}_{3}\right)$ of $\mathbf{6 f f}$

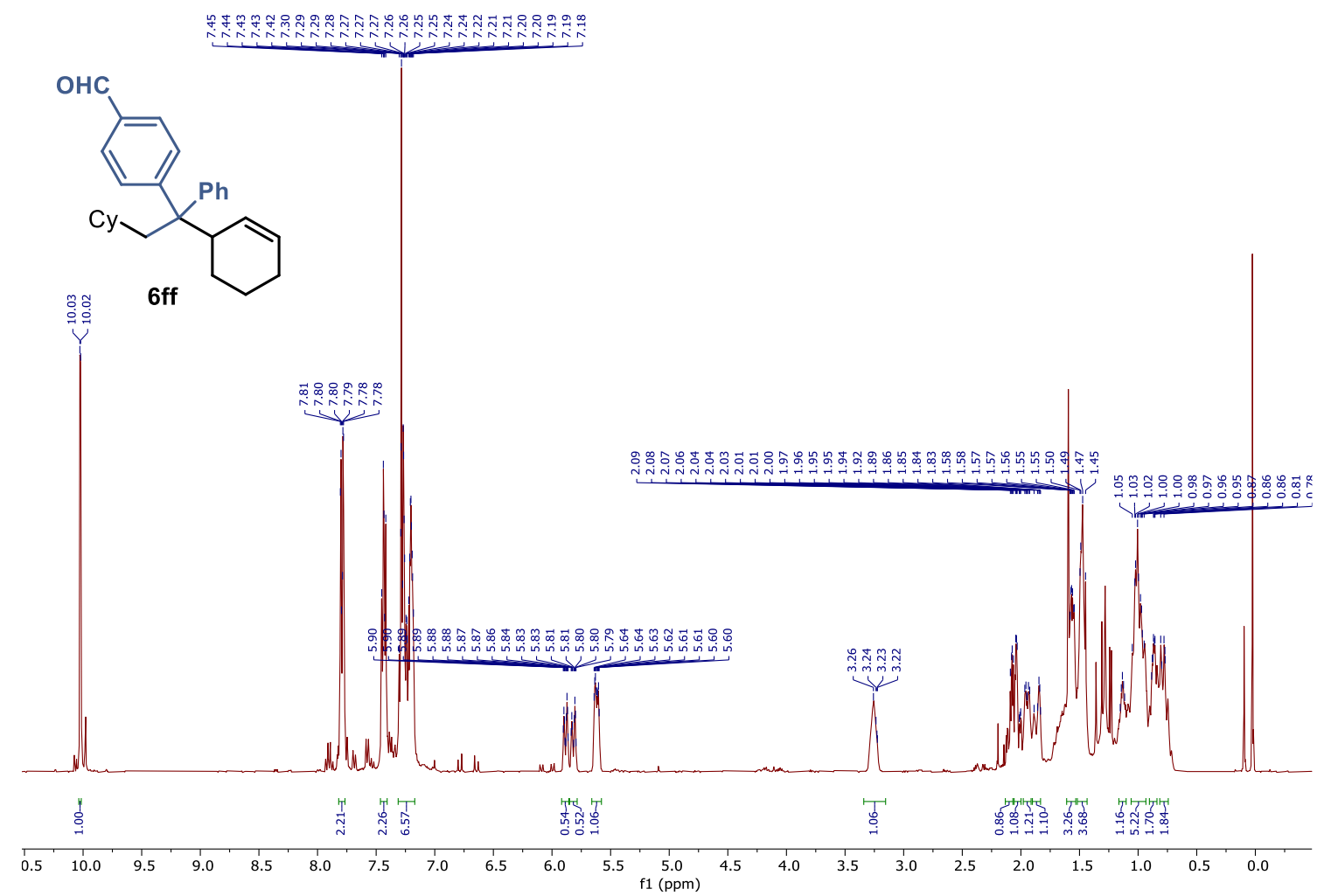

${ }^{13} \mathrm{C}$ NMR $\left(101 \mathrm{MHz}, \mathrm{CDCl}_{3}\right)$ of $\mathbf{6 f f}$ 

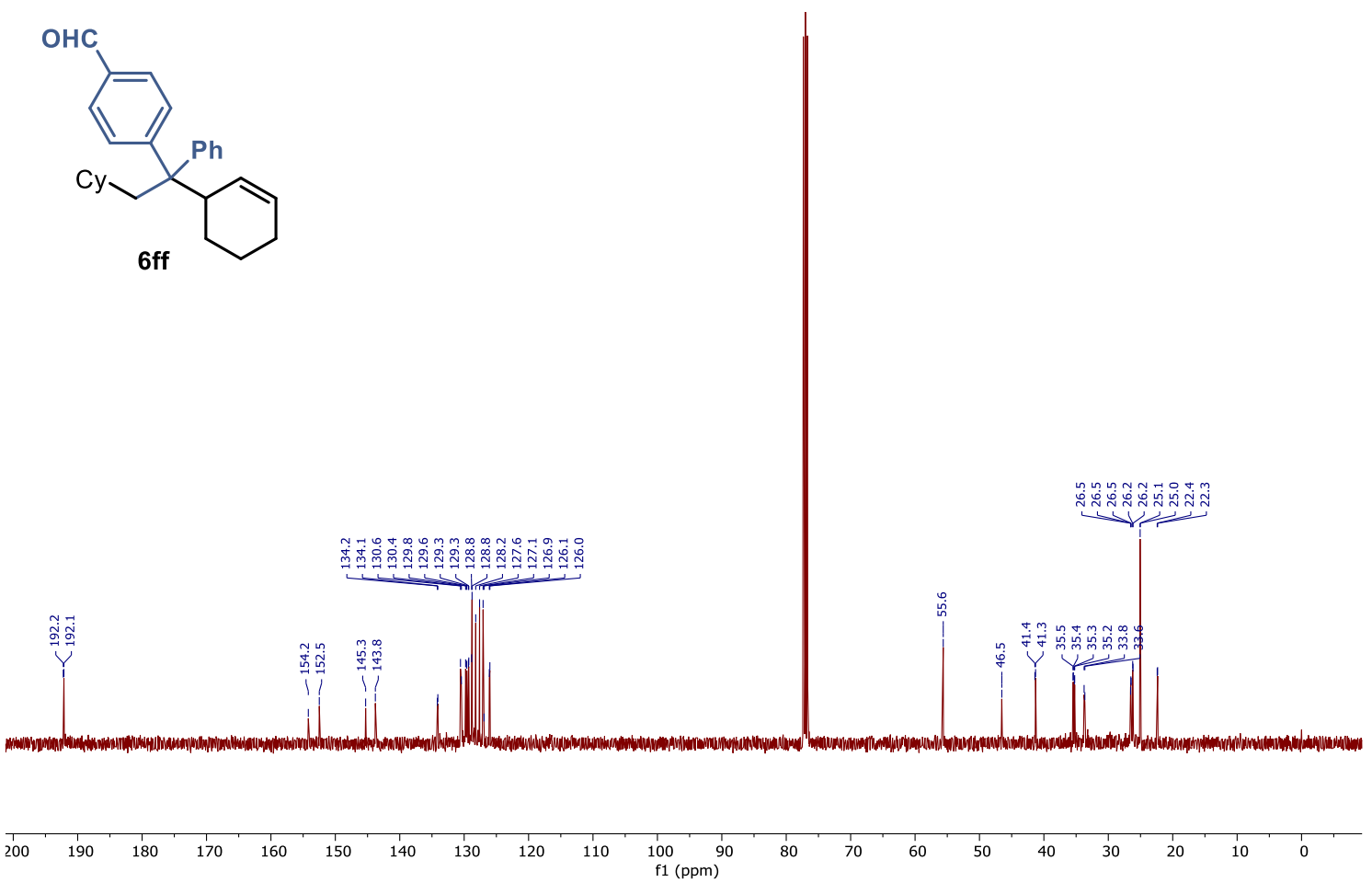

${ }^{1} \mathrm{H}$ NMR $\left(400 \mathrm{MHz}, \mathrm{CDCl}_{3}\right)$ of $\mathbf{6 g g}$
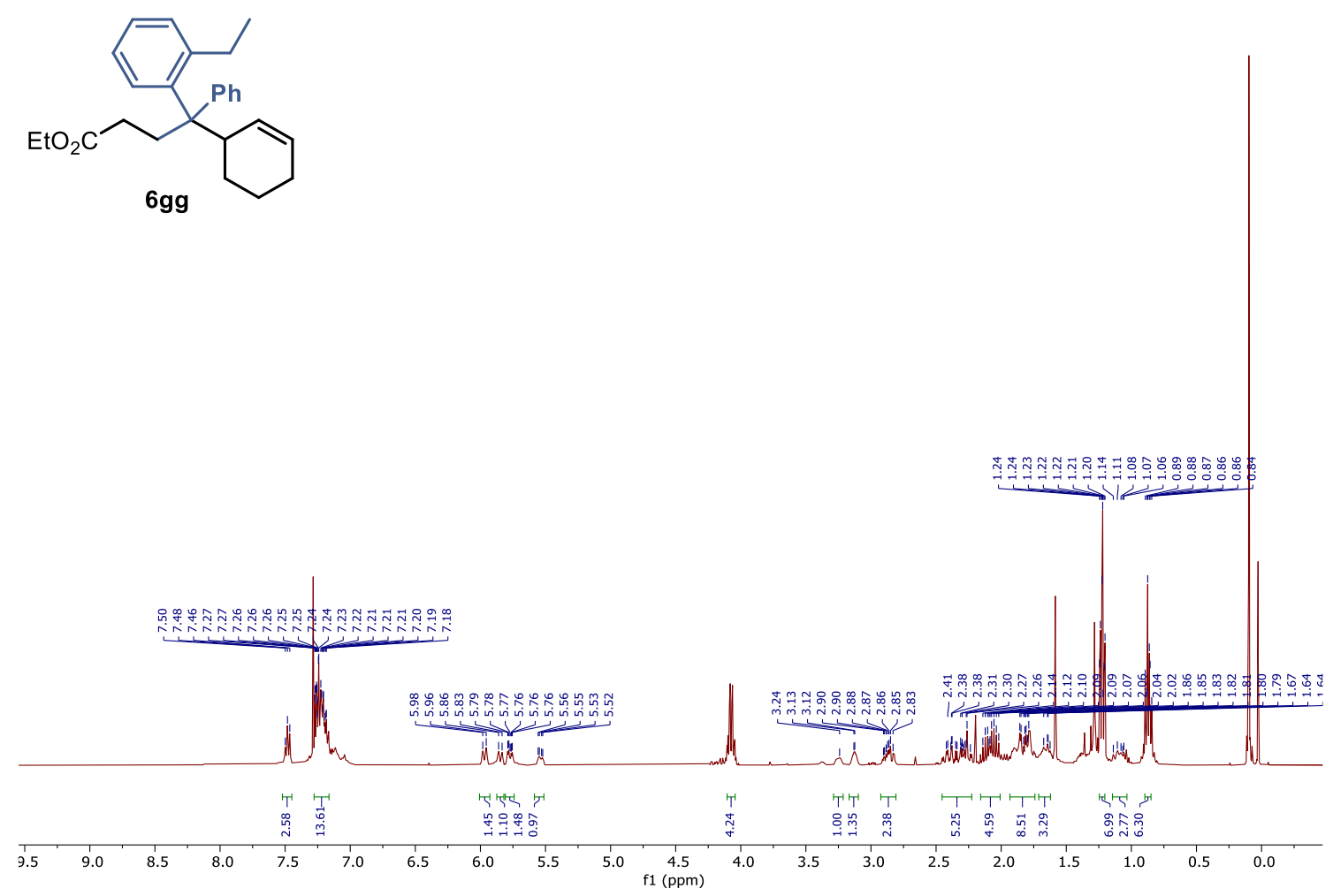
${ }^{13} \mathrm{C}$ NMR (101 MHz, $\left.\mathrm{CDCl}_{3}\right)$ of $\mathbf{6 g g}$

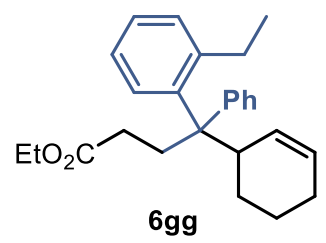

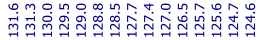

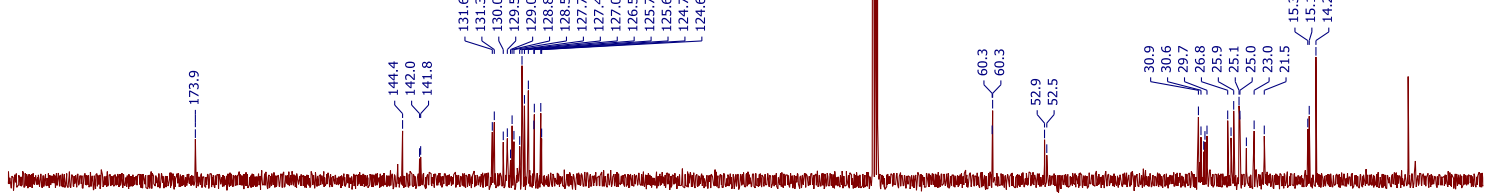

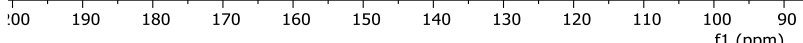

${ }^{1} \mathrm{H}$ NMR (400 MHz, $\mathrm{CDCl}_{3}$ ) of $\mathbf{6 h h}$<smiles>CCOC(=O)CCC(c1ccccc1)(c1ccsc1)C1C=CCCC1</smiles>

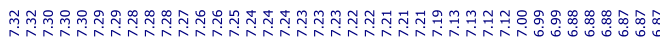

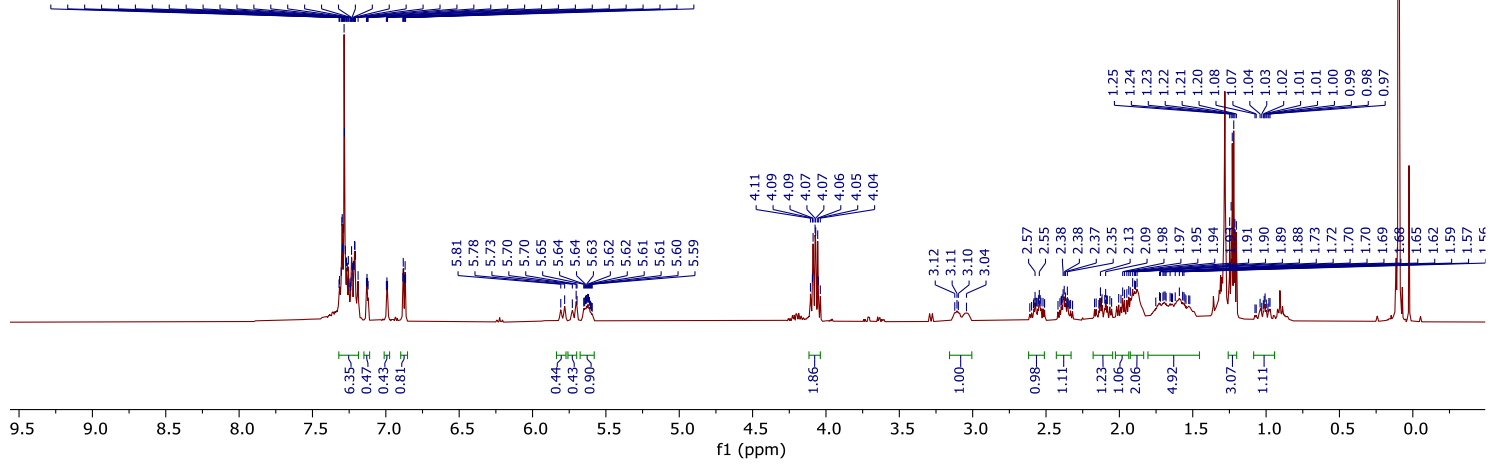


${ }^{13} \mathrm{C}$ NMR (101 MHz, $\left.\mathrm{CDCl}_{3}\right)$ of $\mathbf{6 h h}$

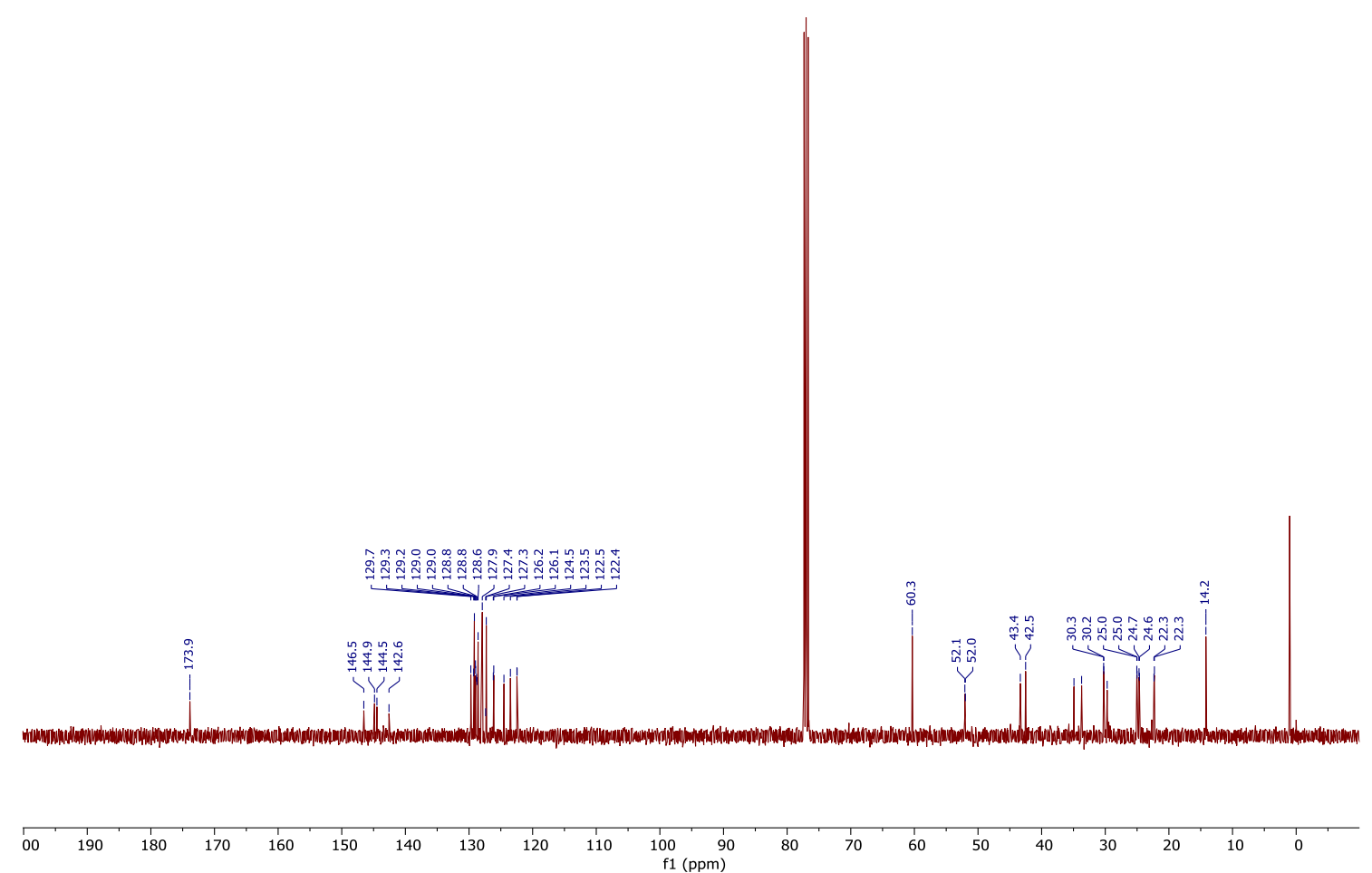

${ }^{1} \mathrm{H} \mathrm{NMR}\left(\mathbf{5 0 0} \mathrm{MHz}, \mathrm{CDCl}_{3}\right)$ of $\mathbf{6 i i}$

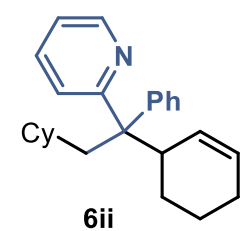

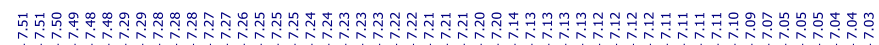

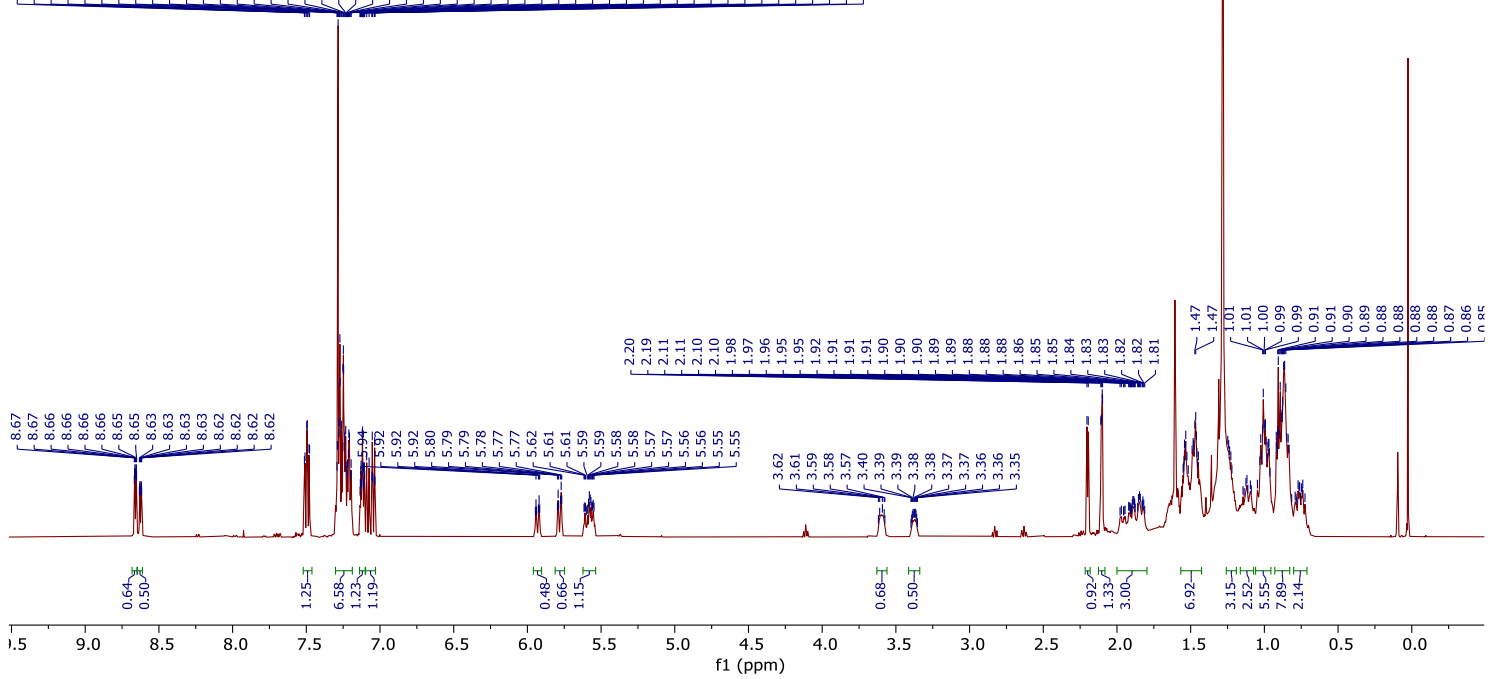


${ }^{13} \mathrm{C}$ NMR $\left(126 \mathrm{MHz}, \mathrm{CDCl}_{3}\right)$ of $\mathbf{6 i i}$<smiles>ClCC(C=C1CCCCC1)(c1ccccn1)c1ccccn1</smiles>
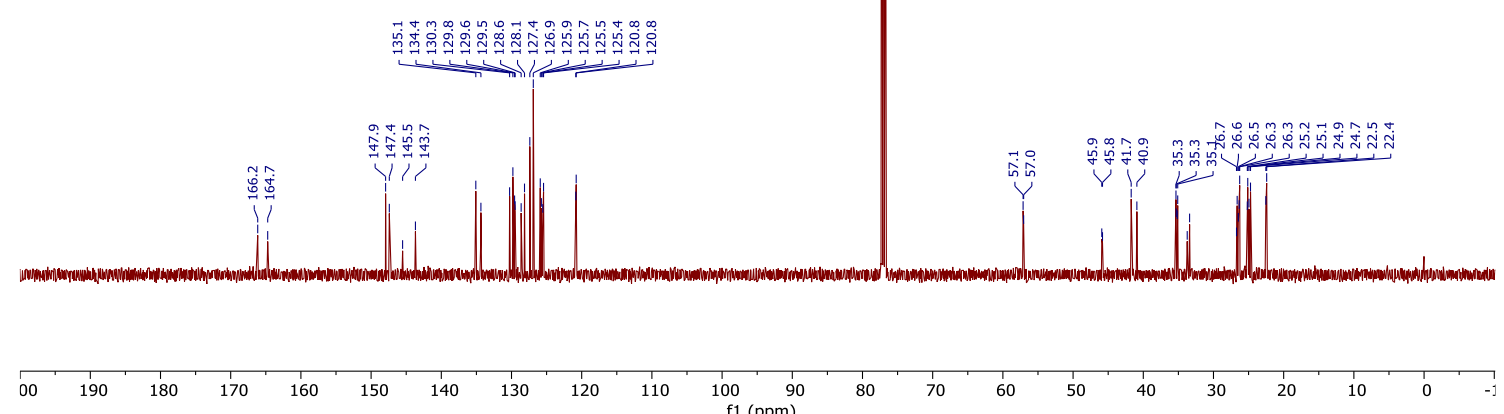

${ }^{1} \mathrm{H} \mathrm{NMR}\left(500 \mathrm{MHz}, \mathrm{CDCl}_{3}\right.$ ) of $\mathbf{6 j j}$

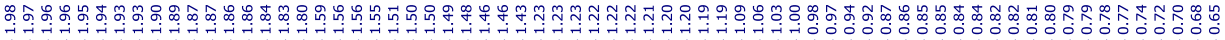<smiles>CC(C)C(=O)OC(C)(C)Oc1ccc(C(CCl)(CCl)C2C=CCCC2)cc1</smiles>

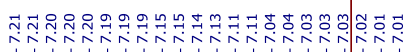

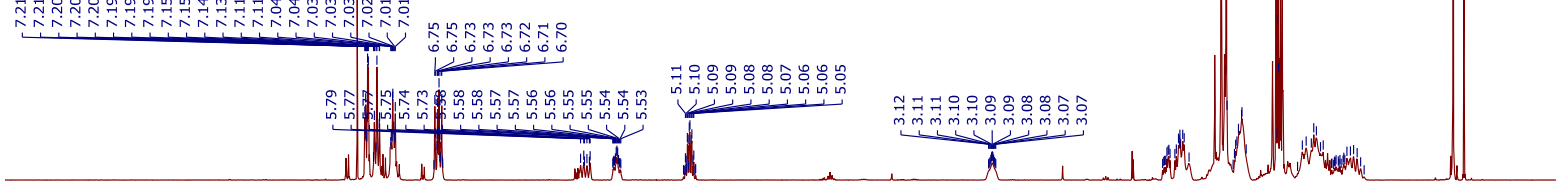

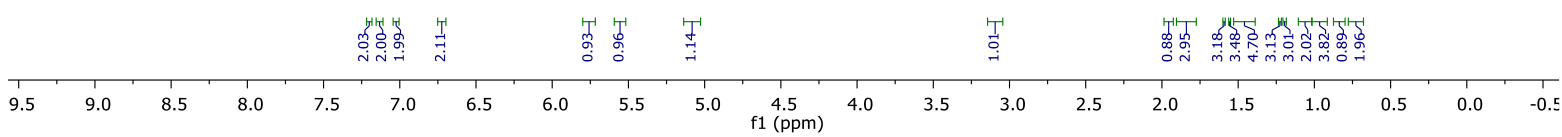


$\left.{ }^{13} \mathrm{C} \mathrm{NMR} \mathrm{(126} \mathrm{MHz,} \mathrm{CDCl}_{3}\right)$ of $\mathbf{6 j j}$<smiles>CC(C)C(=O)OC(C)(C)Oc1ccc(C(CCl)(CCl)C2C=CCCC2)cc1</smiles>

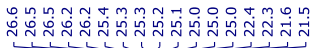

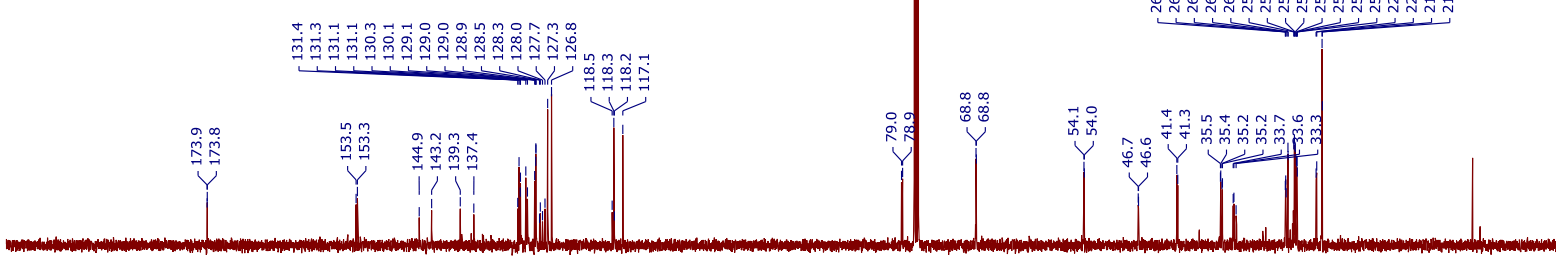

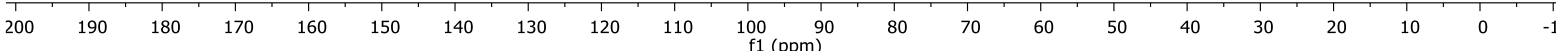


${ }^{1} \mathrm{H}$ NMR $\left(500 \mathrm{MHz}, \mathrm{CDCl}_{3}\right)$ of $\mathbf{6 k k}$

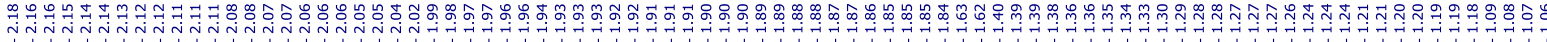

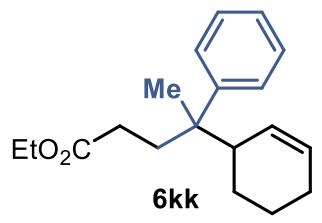

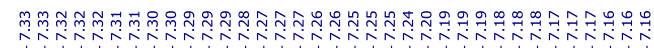
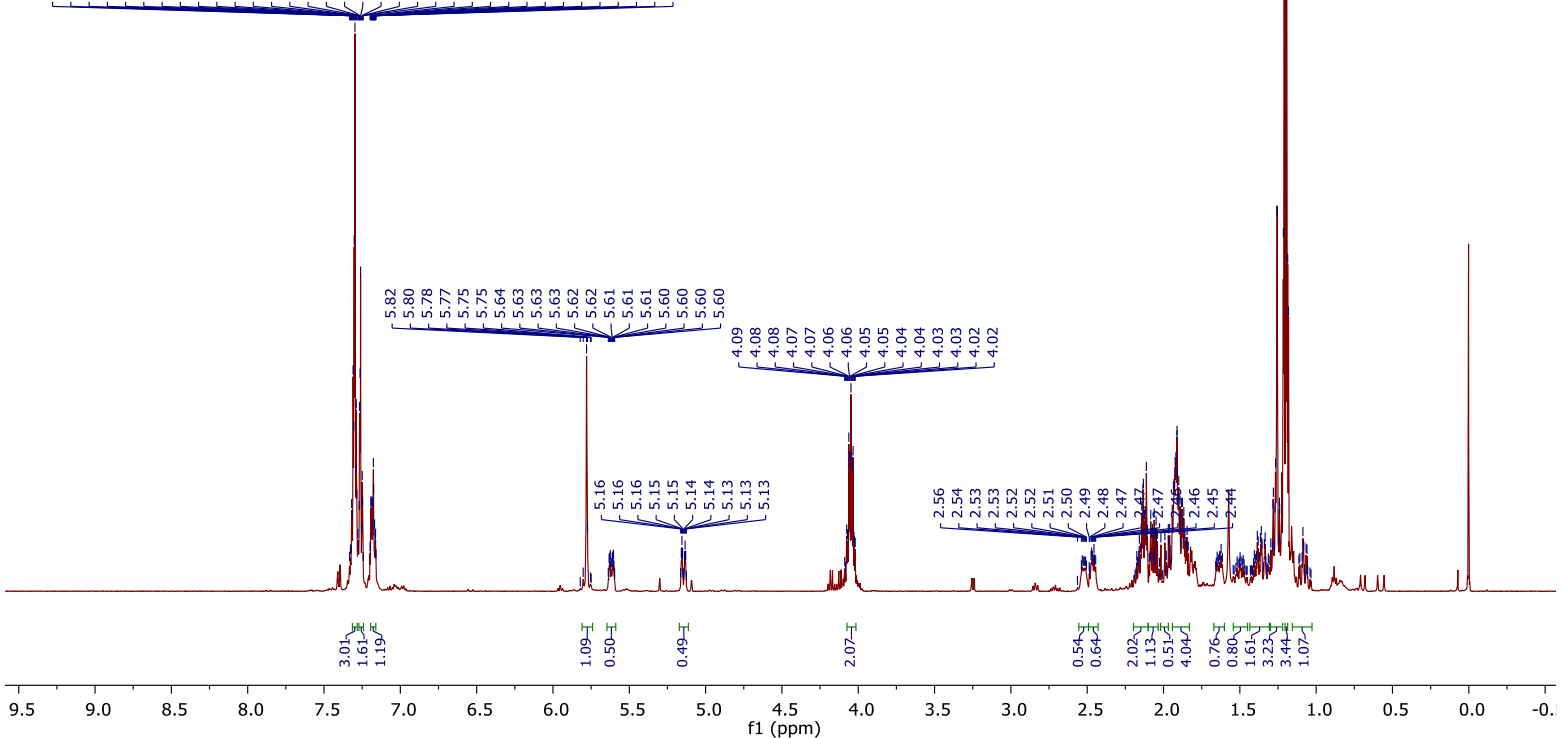

${ }^{13} \mathrm{C} \mathrm{NMR}\left(126 \mathrm{MHz}, \mathrm{CDCl}_{3}\right)$ of $\mathbf{6 k k}$ 

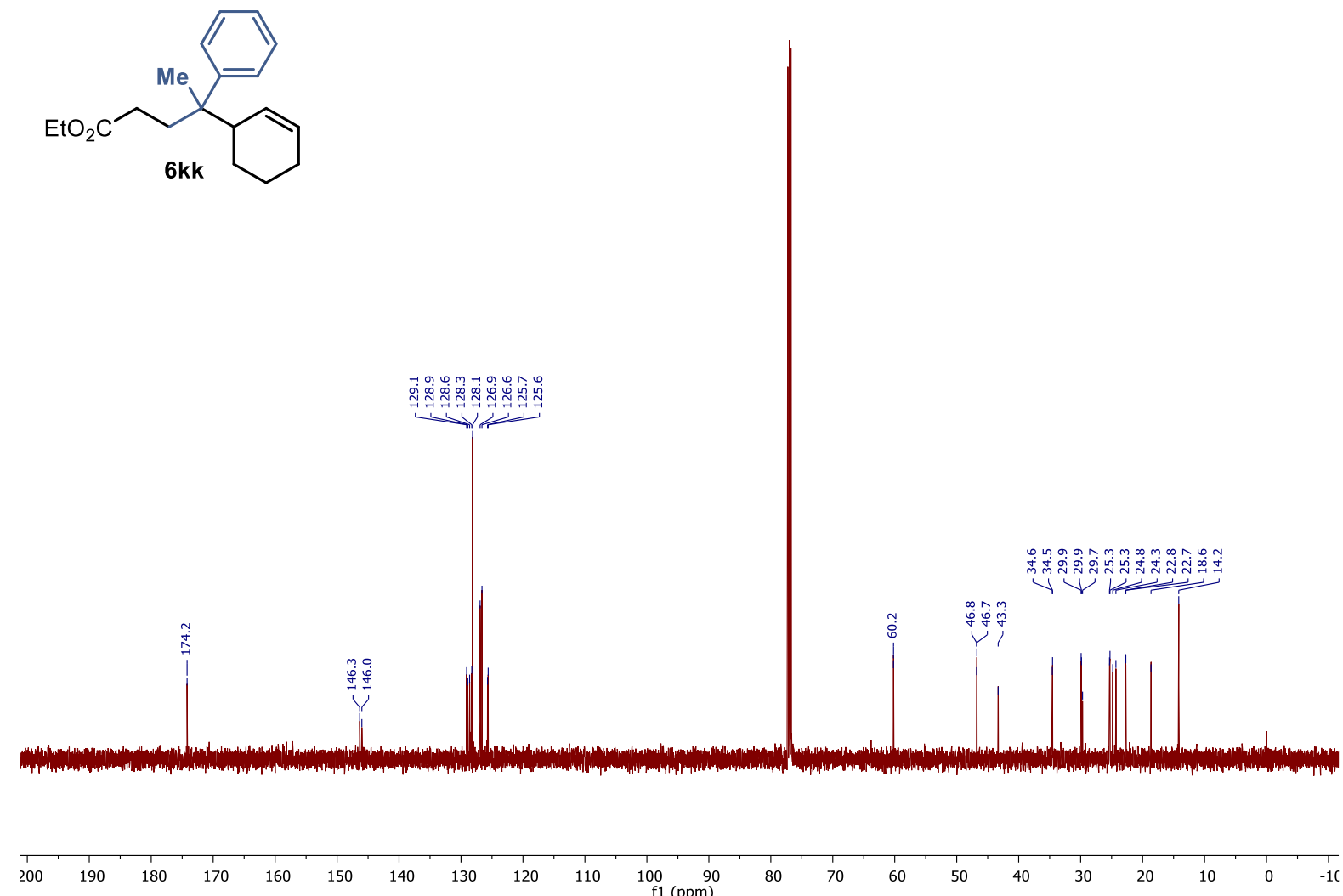

${ }^{1} \mathrm{H}$ NMR $\left(500 \mathrm{MHz}, \mathrm{CDCl}_{3}\right)$ of $\mathbf{6 1 1}$

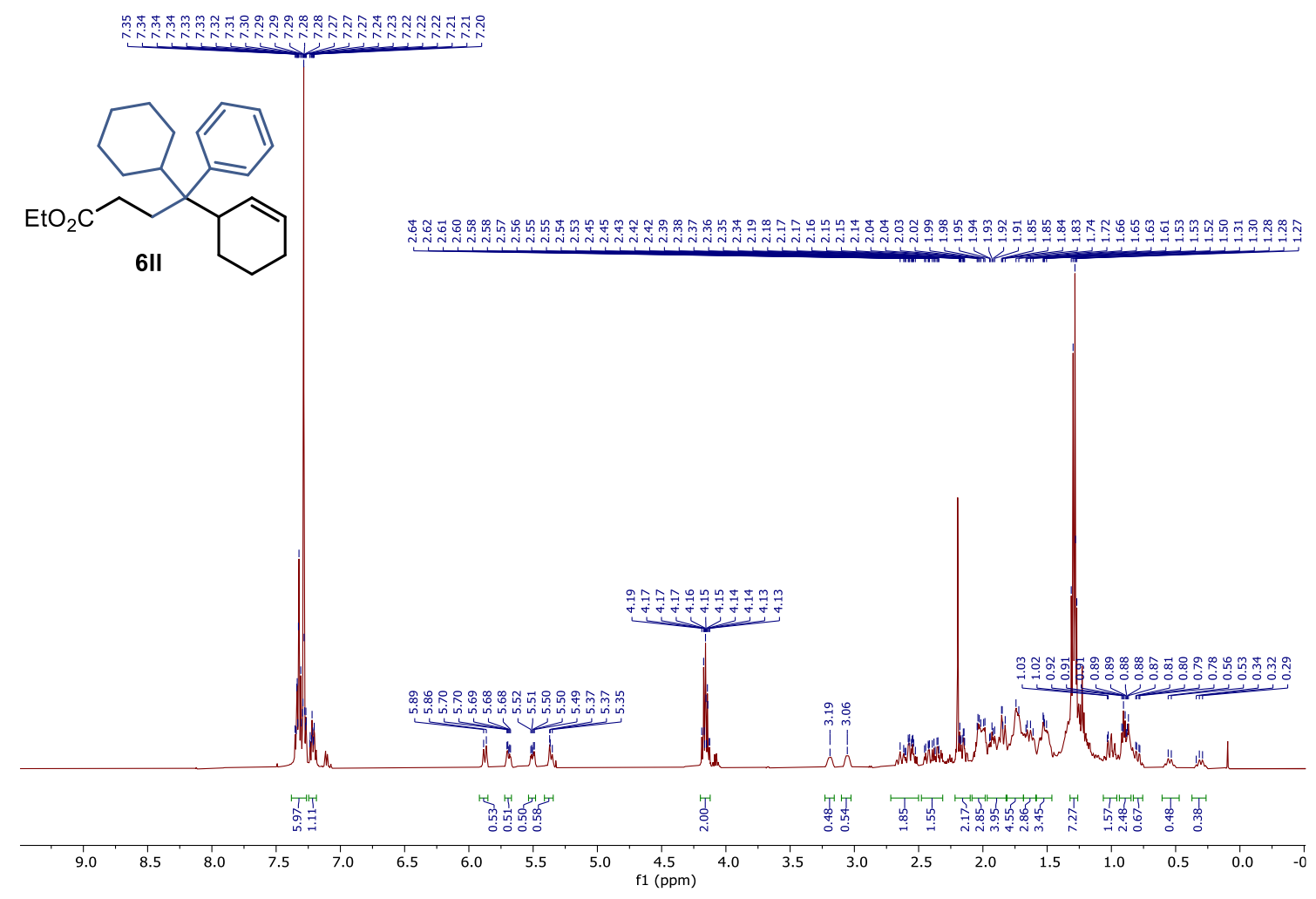

${ }^{13} \mathrm{C}$ NMR (126 MHz, $\mathrm{CDCl}_{3}$ ) of 611 


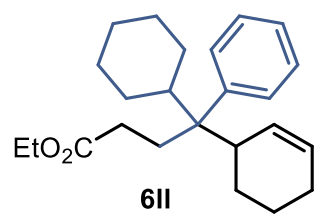

6II

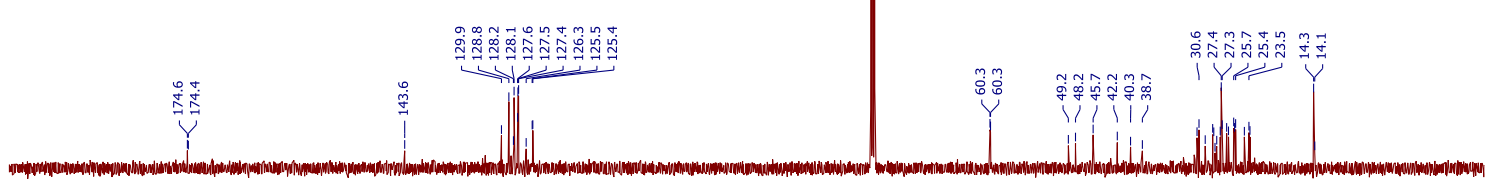

$\begin{array}{llllllllll}190 & 180 & 170 & 160 & 150 & 140 & 130 & 120 & 110 & 100 \\ \mathrm{f} 1(\mathrm{ppm})\end{array}$

${ }^{1} \mathrm{H}$ NMR $\left(500 \mathrm{MHz}, \mathrm{CDCl}_{3}\right)$ of $\mathbf{6 m m}$<smiles>CCOC(=O)CCC(C1C=CCCC1)(C1CCCCC1)C1CCCCC1</smiles>

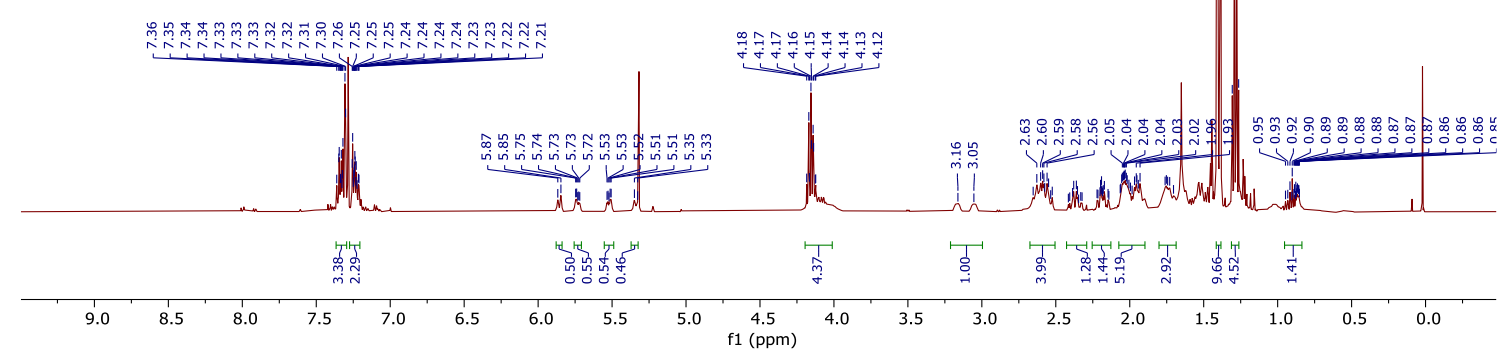


${ }^{13} \mathrm{C}$ NMR $\left(101 \mathrm{MHz}, \mathrm{CDCl}_{3}\right)$ of $\mathbf{6 m m}$<smiles>CCOC(=O)CCC(C1C=CCCC1)(C1CCCCC1)C1CCCCC1</smiles>

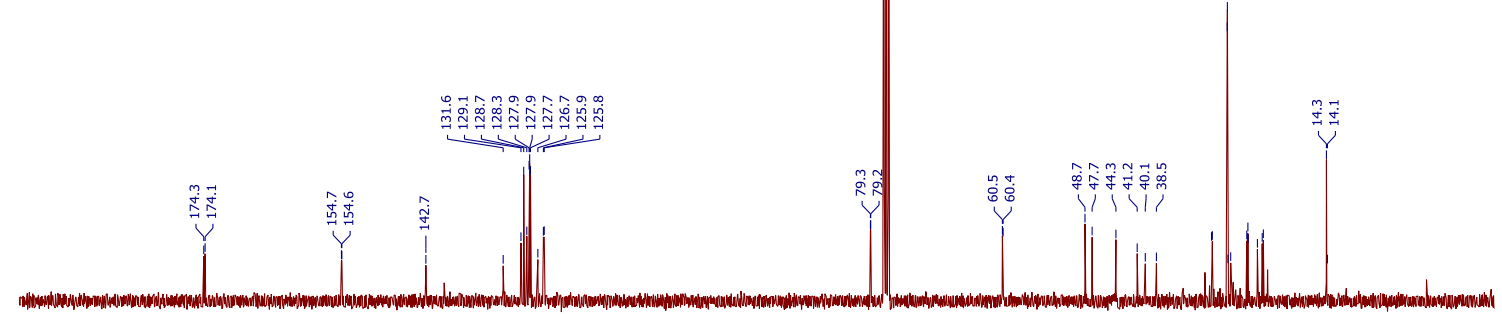

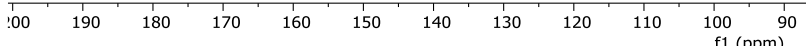

${ }^{1} \mathrm{H}$ NMR (500 MHz, $\left.\mathrm{CDCl}_{3}\right)$ of $\mathbf{6 n n}$

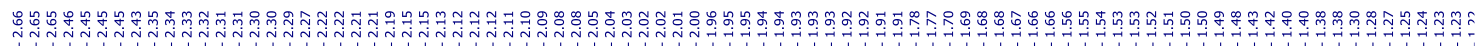<smiles>CCOC(=O)CCC(OC(C)(C)C)(c1ccccc1)C1C=CCCC1</smiles>

$6 \mathrm{nn}$

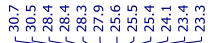


${ }^{13} \mathrm{C}$ NMR (101 MHz, $\mathrm{CDCl}_{3}$ ) of $\mathbf{6 n n}$

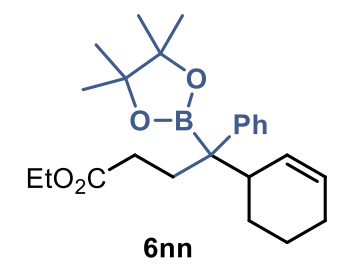

$6 n n$

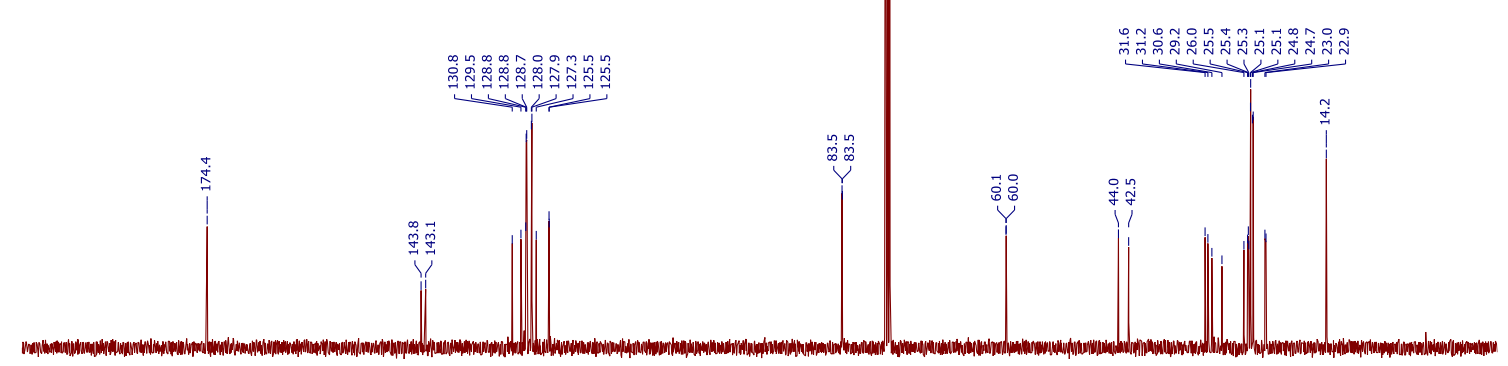

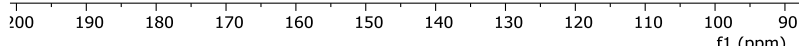

${ }^{1} \mathrm{H}$ NMR $\left(500 \mathrm{MHz}, \mathrm{CDCl}_{3}\right)$ of $\mathbf{6 o o}$<smiles>CCOC(=O)CCC(c1ccccc1)C1C=CCCC1</smiles>

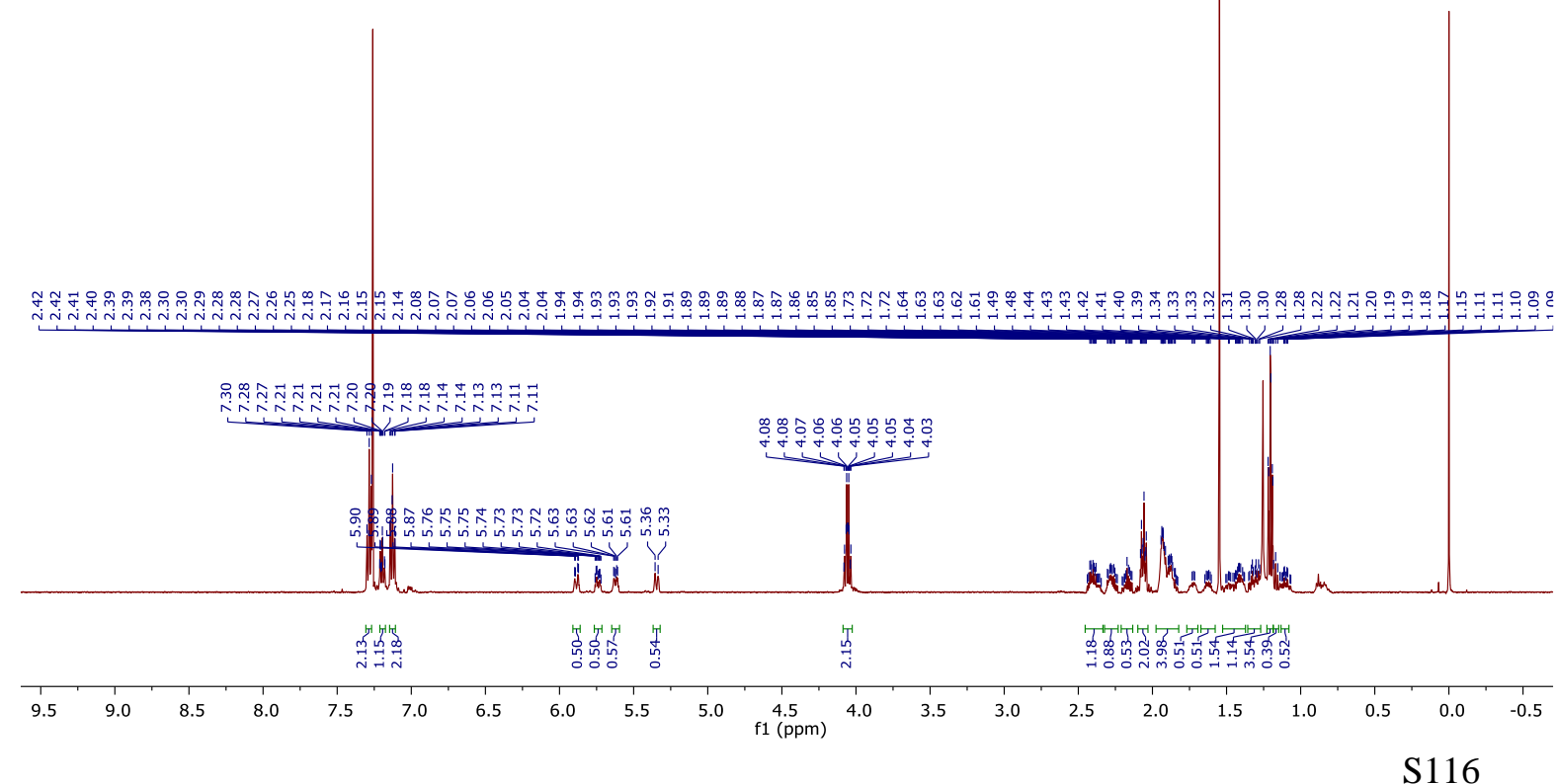


${ }^{13} \mathrm{C}$ NMR $\left(126 \mathrm{MHz}, \mathrm{CDCl}_{3}\right)$ of $\mathbf{6 o o}$<smiles>CCOC(=O)CCC(c1ccccc1)C1C=CCCC1</smiles>

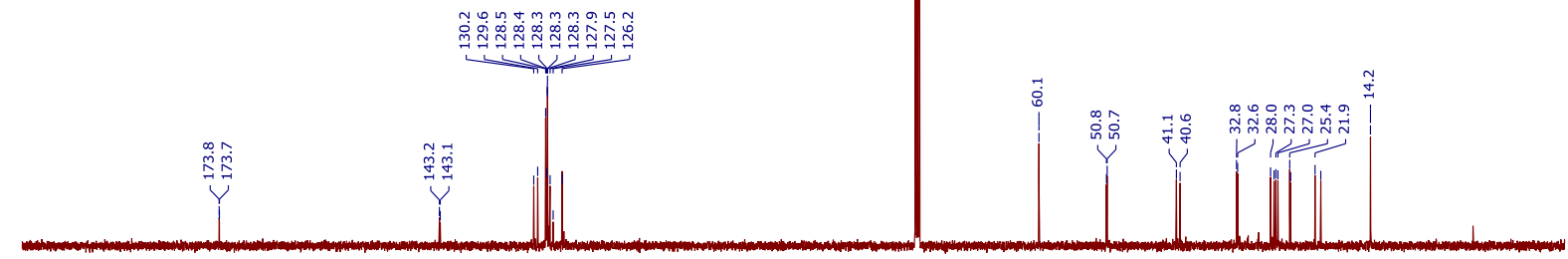

$\begin{array}{lllllllllll}200 & 190 & 180 & 170 & 160 & 150 & 140 & 130 & 120 & 110 & 100 \\ \mathrm{f} 1(\mathrm{ppm})\end{array}$

${ }^{1} \mathrm{H}$ NMR (500 MHz, $\left.\mathrm{CDCl}_{3}\right)$ of $\mathbf{6 p p}$
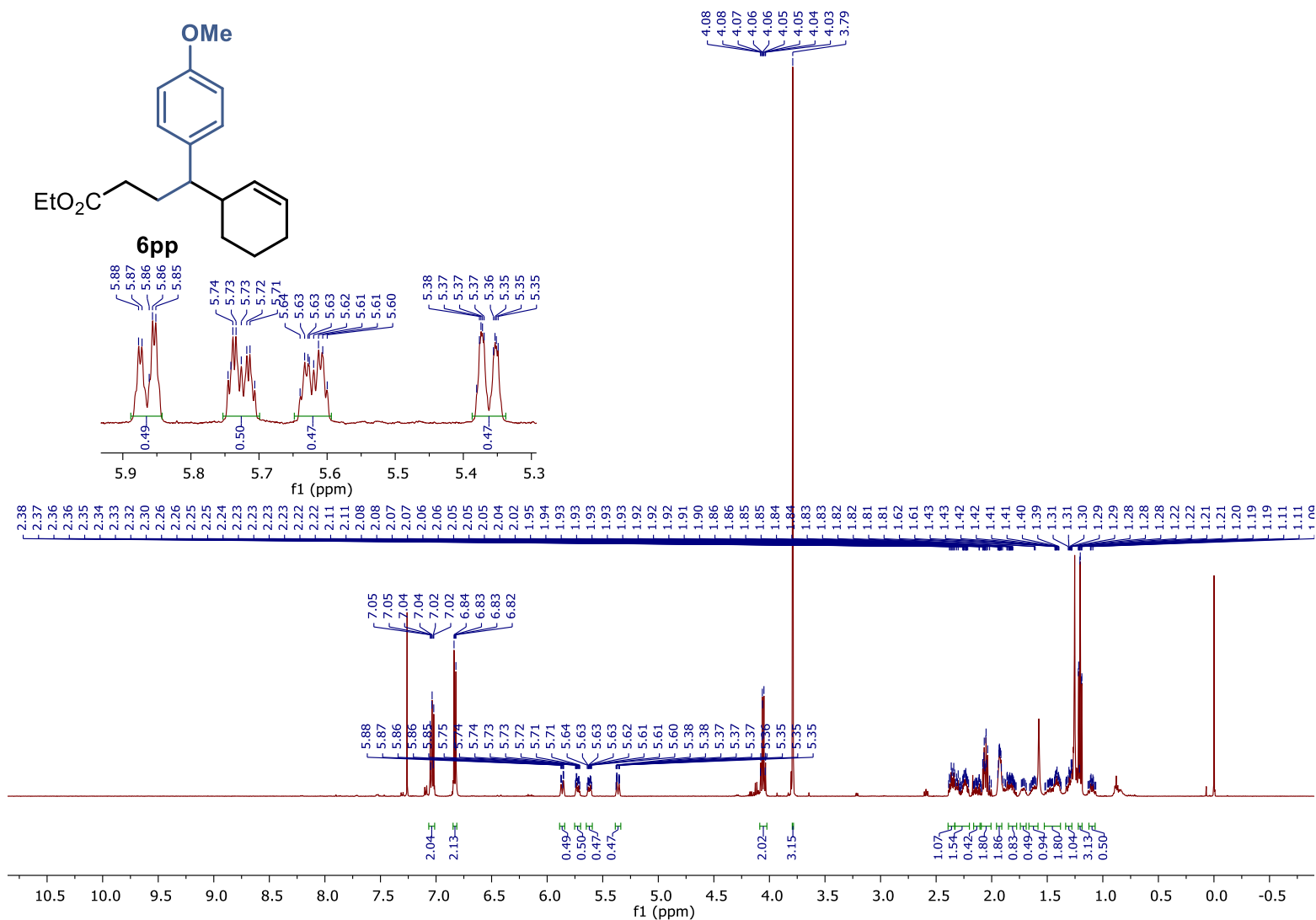
${ }^{13} \mathrm{C}$ NMR (126 MHz, $\mathrm{CDCl}_{3}$ ) of $\mathbf{6 p p}$<smiles>CCOC(=O)CCC(c1ccc(OC)cc1)C1C=CCCC1</smiles>

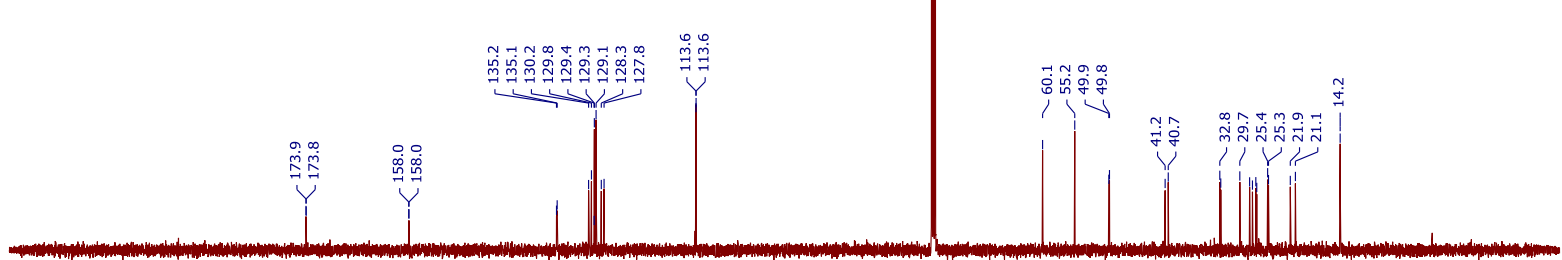

$\begin{array}{lllllllllllllllllllllllllll}210 & 200 & 190 & 180 & 170 & 160 & 150 & 140 & 130 & 120 & 110 & 100 & 90 & 80 & 70 & 60 & 50 & 40 & 30 & 20 & 10 & 0 & -10\end{array}$ 